\title{
Quantum Squeezing of Motion in a Mechanical Resonator
}

\author{
Thesis by \\ Emma E. Wollman \\ In Partial Fulfillment of the Requirements \\ for the Degree of \\ Doctor of Philosophy
}

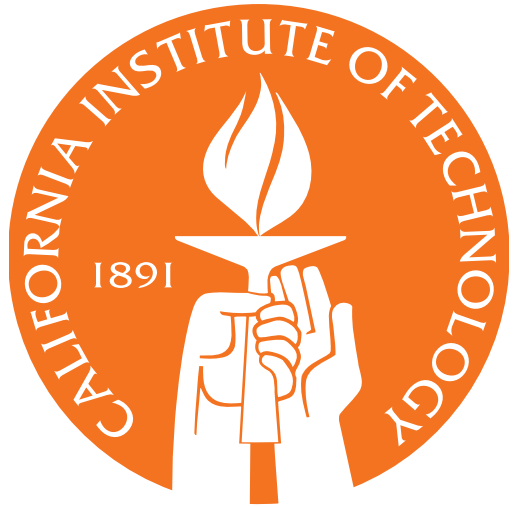

California Institute of Technology

Pasadena, California

2015

(Defended May 28, 2015) 
(C) 2015

Emma E. Wollman

All Rights Reserved 


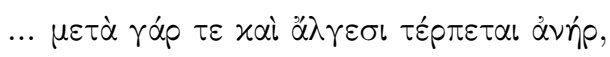

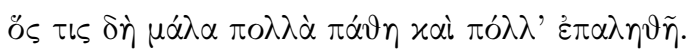

- Homer 


\section{Acknowledgments}

First, I'd like to thank my advisor, Keith Schwab. From teaching me how to drill a simple hole in my first year at Caltech to helping me with paper-writing and career-building in my sixth, Keith always showed patience and understanding. He gave me the opportunity to work on a variety of interesting projects, from graphene, to superconducting amplifiers, to the results discussed here. Most of all, Keith taught me to be more decisive, to take more risks, and to do whatever it takes.

I'd also like to thank the other members of the Schwab group. Junho Suh was fundamental in setting up the electromechanics experiments at Caltech before I joined the team. He also provided an excellent example of how to stay calm and keep a positive attitude in all situations. Matt Shaw, Kin Chung Fong, Laura de Lorenzo, Ari Weinstein, Chan U Lei, Harish Ravi, and Aaron Pearlman all greatly influenced my time at Caltech. They were always willing to stop what they were doing to help out, whether it was lifting the cans on the cryostats, or discussing the latest odd device behavior.

Our theory collaborators on the squeezing project, Aashish Clerk, Florian Marquardt, and especially Andreas Kronwald, gave essential support before, during, and after measurement-taking. Although they sometimes seemed puzzled at the number of ways that things can go wrong in experimental work, they showed great patience as we tried to make their proposal a reality.

I couldn't have made it through grad school without the support of my friends: Paul, Branimir, Helge, Alice, Tristan, Chris, Kris, Doron, Mo, and Carly. They gave me something to look forward to throughout the week, and something to talk about other than grad school.

I feel like my family has been with me every step of the way throughout graduate school. During our weekly phone conversations, my parents would sometimes seem more upset than I was when the experiments had yet again taken a turn for the worse. I also credit them for instilling a love of math and science in me from a young age; I have fond memories of doing fraction-based scavenger hunts in the library of my elementary school with my mother and of growing arrays of multi-colored crystals dyed with food coloring with my father.

Lastly, I'd like to thank my partner, Seán Meenehan. I can't describe how much your support has meant to me throughout these past few years. Grad school was worth it, if only because I met you. 


\section{Abstract}

Quantum mechanics places limits on the minimum energy of a harmonic oscillator via the everpresent "zero-point" fluctuations of the quantum ground state. Through squeezing, however, it is possible to decrease the noise of a single motional quadrature below the zero-point level as long as noise is added to the orthogonal quadrature. While squeezing below the quantum noise level was achieved decades ago with light, quantum squeezing of the motion of a mechanical resonator is a more difficult prospect due to the large thermal occupations of megahertz-frequency mechanical devices even at typical dilution refrigerator temperatures of $\sim 10 \mathrm{mK}$.

Kronwald, Marquardt, and Clerk [30] propose a method of squeezing a single quadrature of mechanical motion below the level of its zero-point fluctuations, even when the mechanics starts out with a large thermal occupation. The scheme operates under the framework of cavity optomechanics, where an optical or microwave cavity is coupled to the mechanics in order to control and read out the mechanical state. In the proposal, two pump tones are applied to the cavity, each detuned from the cavity resonance by the mechanical frequency. The pump tones establish and couple the mechanics to a squeezed reservoir, producing arbitrarily-large, steady-state squeezing of the mechanical motion. In this dissertation, I describe two experiments related to the implementation of this proposal in an electromechanical system. I also expand on the theory presented in [30] to include the effects of squeezing in the presence of classical microwave noise, and without assumptions of perfect alignment of the pump frequencies.

In the first experiment, we produce a squeezed thermal state using the method of Kronwald et. al.. We perform back-action evading measurements of the mechanical squeezed state in order to probe the noise in both quadratures of the mechanics. Using this method, we detect single-quadrature fluctuations at the level of $1.09 \pm 0.06$ times the quantum zero-point motion.

In the second experiment, we measure the spectral noise of the microwave cavity in the presence of the squeezing tones and fit a full model to the spectrum in order to deduce a quadrature variance of $0.80 \pm 0.03$ times the zero-point level. These measurements provide the first evidence of quantum squeezing of motion in a mechanical resonator. 


\section{Contents}

Acknowledgments $\quad$ iv

$\begin{array}{ll}\text { Abstract } & \text { v }\end{array}$

1 Introduction 1

2 Theory $\quad 8$

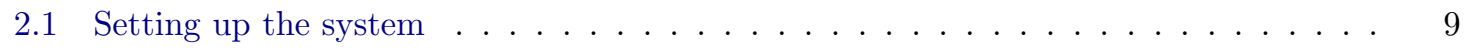

2.1 .1 Parameters of the system $\ldots \ldots \ldots \ldots \ldots$

2.1 .2 Input noise operators $\ldots \ldots \ldots \ldots \ldots$

2.1 .3 Pump configuration . . . . . . . . . . . . . . . . 11

2.2 Hamiltonian . . . . . . . . . . . . . . . . . . . . . . . . . . 11

2.2 .1 Optomechanical Hamiltonian . . . . . . . . . . . . . . . . . . . . . 11

2.2 .2 Rotating frame . . . . . . . . . . . . . . . . . . . . . . . 12

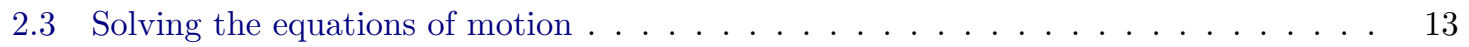

2.3 .1 Classical solution . . . . . . . . . . . . . . . . . . . . 13

2.3.2 Rotating wave approximation . . . . . . . . . . . . . . 14

2.3.3 Heisenberg-Langevin equations . . . . . . . . . . . . . . . . 15

2.4 Quadrature squeezing . . . . . . . . . . . . . . . . . 16

2.4.1 Quadrature operators . . . . . . . . . . . . . . . . . 16

2.4.2 Frequency-domain Langevin equations . . . . . . . . . . . . . . . . . 16

2.4.3 Special case: $\Delta=\delta=0 \ldots \ldots \ldots \ldots \ldots \ldots \ldots \ldots$

2.4 .4 General case . . . . . . . . . . . . . . . . . . . . . . . 19

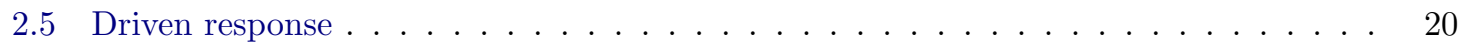

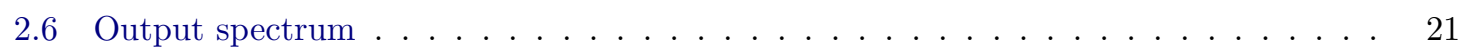

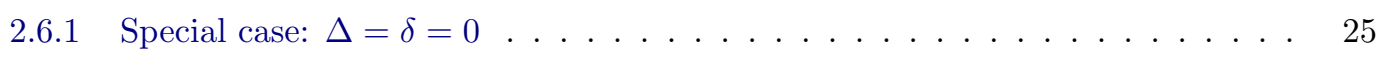

2.6 .2 Weak driving regime . . . . . . . . . . . . . . . . . 25

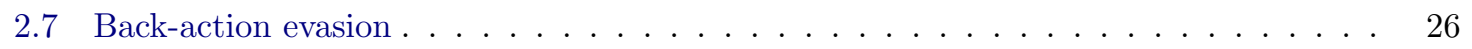

2.7.1 Balanced back-action with $\Delta=0 \ldots \ldots \ldots \ldots \ldots$ 
2.7.2 Effects of imbalance . . . . . . . . . . . . . . . . . . 27

2.8 Single, red-detuned tone . . . . . . . . . . . . . . . . . . . . . . . . . . . . . . 29

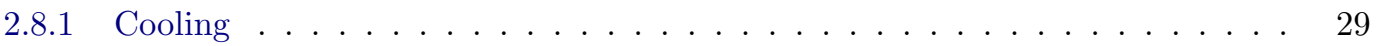

2.8 Driven response . . . . . . . . . . . . . . . . . 30

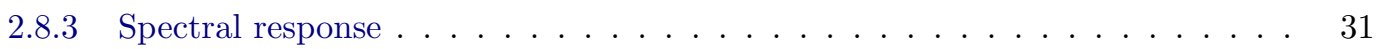

3 Experimental set-up and calibrations 33

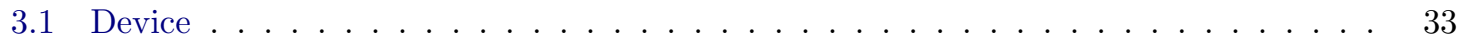

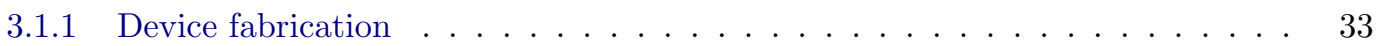

3.1.2 Sample package and shielding . . . . . . . . . . . . . . . 35

3.2 Dilution refrigerator .......................... 35

3.3 Microwave circuitry ........................... 36

3.3.1 Input line: sources, filtering, and attenuation . . . . . . . . . . . . 37

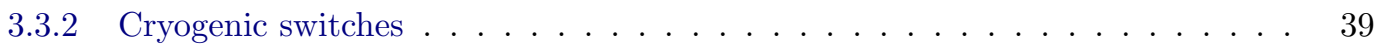

3.3.3 Output line: circulators and amplifiers . . . . . . . . . . . . . . 40

3.4 Device characterization and calibrations . . . . . . . . . . . . . . . . . . . . 42

3.4.1 Parasitic channel ....................... 42

3.4 Thermal calibration ...................... 43

3.4.3 Linewidth broadening and pump power calibration . . . . . . . . . . . . . 44

4 Device 1: Back-action evading measurements for direct detection of squeezed motion
45

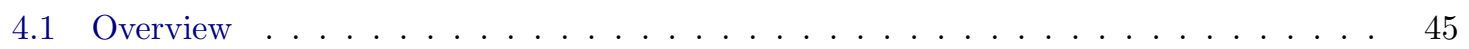

4.2 Device calibrations and characterizations . . . . . . . . . . . . . . . . . . . 47

4.2.1 Thermal calibration ...................... . . 47

4.2 .2 Linewidth broadening . . . . . . . . . . . . . . . . 49

4.2.3 Balancing calibration ...................... 51

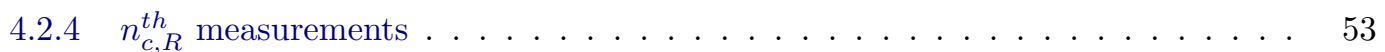

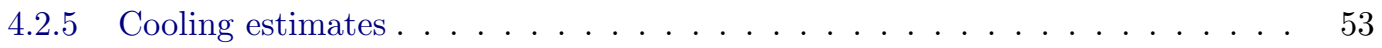

4.3 BAE probe measurements . . . . . . . . . . . . . . . . . . . . . . 55

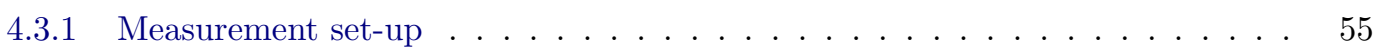

4.3 .2 Data processing. . . . . . . . . . . . . . . . . 58

4.3.3 Parameter search ....................... 61

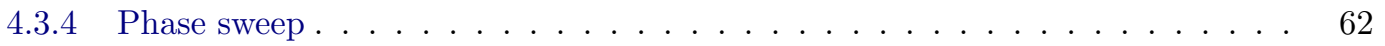

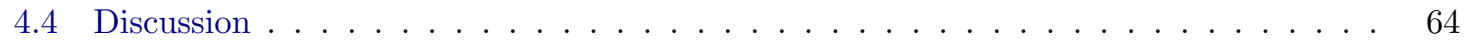


5 Device 2: Full-spectrum measurements $\quad 65$

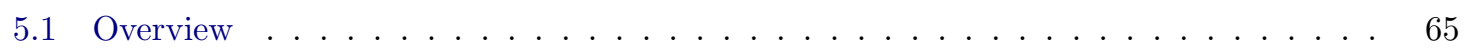

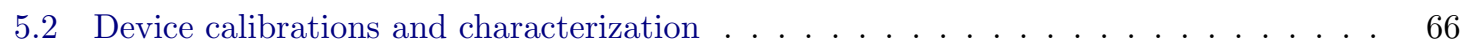

5.2 .1 Thermal calibration . . . . . . . . . . . . . . 66

5.2 .2 Linewidth broadening . . . . . . . . . . . . . . . 68

5.2 .3 Noise floor . . . . . . . . . . . . . . . . . . . . . . . . 70

5.2 .4 Cooling measurements . . . . . . . . . . . . . . . . . . . 72

$5.2 .5 \quad$ Excess amplifier phase noise . . . . . . . . . . . . . . . . . . . 72

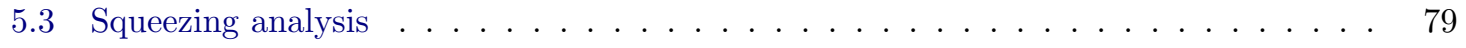

5.3 .1 Procedure . . . . . . . . . . . . . . . . . . . 79

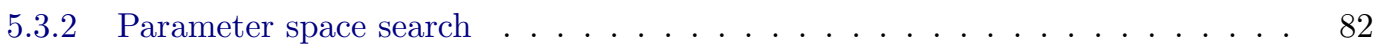

5.3 .3 Alignment . . . . . . . . . . . . . . . . . . . 82

$5.3 .4 \quad$ Fit results: ideal assumptions . . . . . . . . . . . . . . . . . . 84

5.3.5 Fit results: including alignment and bad cavity effects . . . . . . . . . . . 87

5.4 Discussion . . . . . . . . . . . . . . . . . . . . . . . . . 90

$\begin{array}{ll}\text { A Table of variable definitions } & 91\end{array}$

$\begin{array}{ll}\text { B Power-dependent heating } & 93\end{array}$

B.1 $n_{c}^{t h}$ heating in the absence of mechanics . . . . . . . . . . . . 93

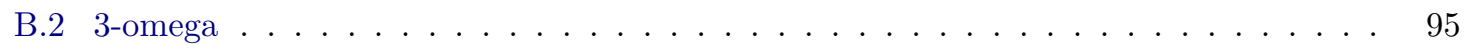

$\begin{array}{ll}\text { Bibliography } & 99\end{array}$ 


\section{List of Figures}

1.1 Quantum noise limits on continuous position measurements. . . . . . . . . . . . 4

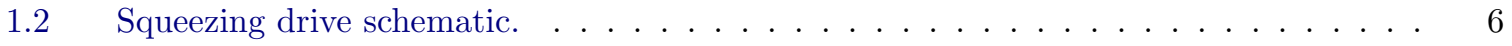

2.1 Definition of coupling rates and mode operators. . . . . . . . . . . . . . 9

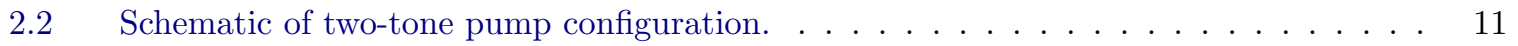

$2.3 \quad$ Model for $\left\langle\hat{X}_{1}^{2}\right\rangle$ at different pump powers and ratios. . . . . . . . . . . . . . . . 19

2.4 Predicted effects of detuning on $\left\langle\hat{X}_{1}^{2}\right\rangle \ldots \ldots \ldots \ldots \ldots \ldots$

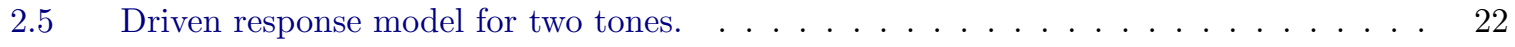

2.6 Spectral response model for two tones. . . . . . . . . . . . . . . . . . . . . 24

2.7 Driven response model for single red-detuned tone. . . . . . . . . . . . . . . 30

2.8 Spectral response model for single red-detuned tone . . . . . . . . . . . . . . . . 31

3.1 Images of a typical device. $\ldots \ldots \ldots \ldots \ldots \ldots \ldots \ldots \ldots \ldots \ldots$

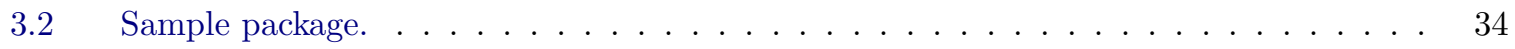

$3.3 \quad$ Additional shielding. . . . . . . . . . . . . . . . . . . . . . 35

3.4 Samples mounted in the fridge. . . . . . . . . . . . . . . . . 36

$3.5 \quad$ Microwave circuit diagram. . . . . . . . . . . . . . . . . . . . . 37

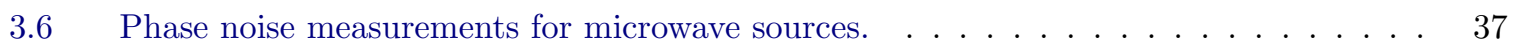

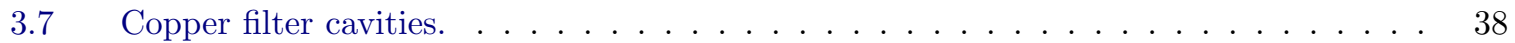

$3.8 \quad$ Fridge circuitry comparison for $\mathrm{D} 1$ and $\mathrm{D} 2 \ldots \ldots \ldots \ldots \ldots$

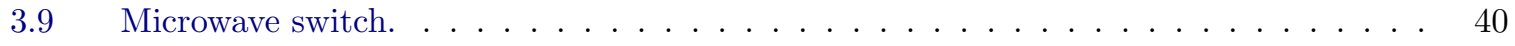

3.10 System noise temperature measurement. . . . . . . . . . . . . . . . . . . . . 41

3.11 Measurements of parasitic bypass channel. . . . . . . . . . . . . . . . . . . . . 42

3.12 Bypass channel model. . . . . . . . . . . . . . . . . . . . . . . . . . . . 43

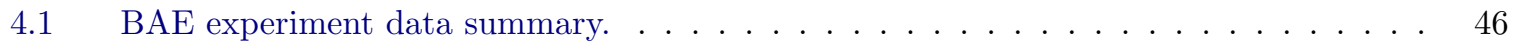

4.2 Pump scheme for BAE measurements of the squeezed state. . . . . . . . . . . . 47

4.3 Pump and sideband scheme for D1's thermal calibration. . . . . . . . . . . . 48

4.4 Thermal calibration for $\mathrm{D} 1 \ldots \ldots \ldots \ldots \ldots$

$4.5 \quad \mathrm{D} 1$ frequencies and linewidths vs temperature. . . . . . . . . . . . . . 50 


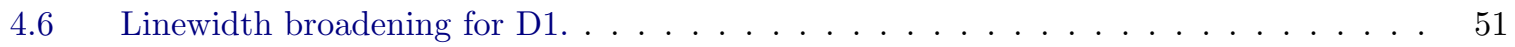

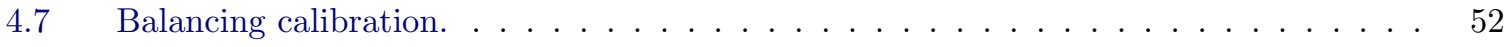

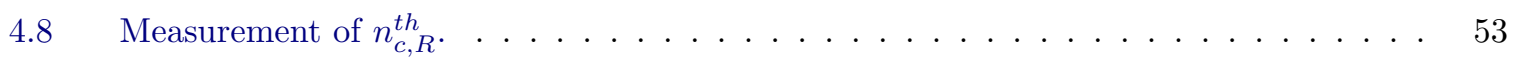

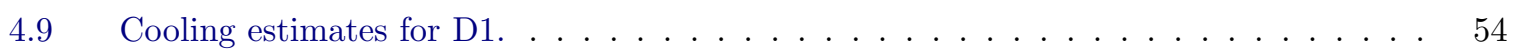

4.10 Phase space description of BAE probe measurements. . . . . . . . . . . . . . . 56

4.11 Circuit diagram for phase measurements. . . . . . . . . . . . . . . . . . 57

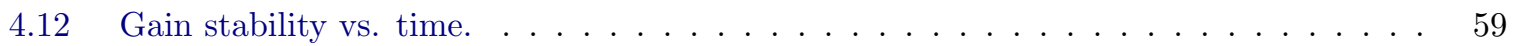

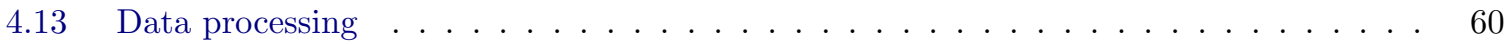

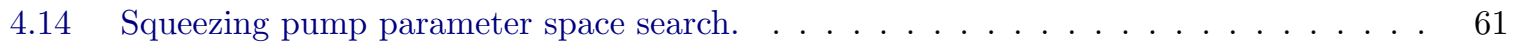

4.15 BAE probe spectra. . . . . . . . . . . . . . . . . . . . 62

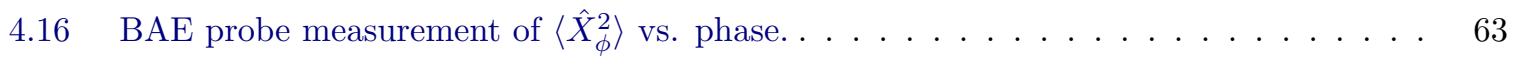

$5.1 \quad$ Thermal calibration for $\mathrm{D} 2 \ldots \ldots \ldots \ldots \ldots \ldots \ldots$

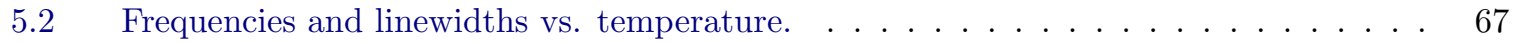

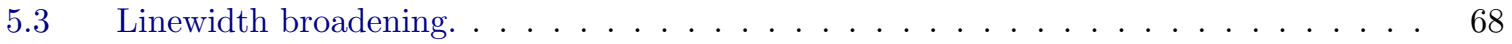

$5.4 \quad$ Frequencies and linewidths vs. red-detuned pump power. . . . . . . . . . . . . 69

$5.5 \quad$ Noise floor analysis . . . . . . . . . . . . . . . . . . . . . . 71

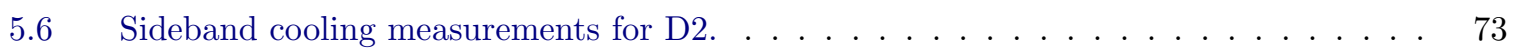

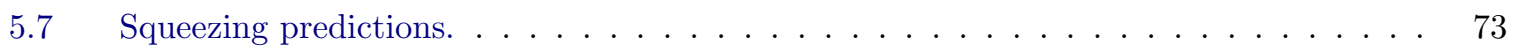

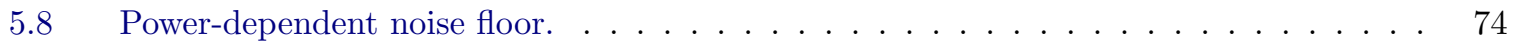

5.9 Signatures of insufficient source phase-noise filtering in a prior device. . . . . . . . . 75

5.10 Effects of filtering the source noise on the power-dependent noise floor. . . . . . . . 75

5.11 Model of room-temperature output line noise sources. . . . . . . . . . . . . . 76

5.12 Results of room-temperature filtering on the output line phase noise. . . . . . . . . 77

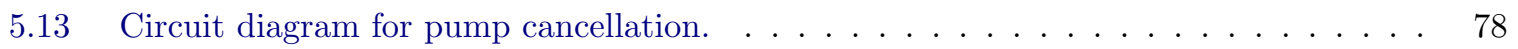

5.14 Results of room-temperature cancellation on the output line phase noise. . . . . . . 78

5.15 Driven response fitting for squeezing measurements. . . . . . . . . . . . . . . . . . . 80

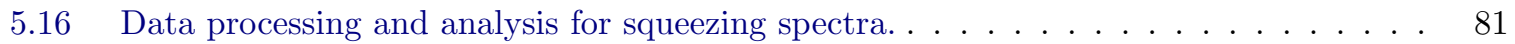

5.17 Search of squeezing pump parameter space. . . . . . . . . . . . . . . . 83

5.18 Frequency alignment for squeezing tones. . . . . . . . . . . . . . . . . 84

5.19 Driven and spectral squeezing responses. . . . . . . . . . . . . . . 85

5.20 Bath occupations vs. pump ratio under ideal assumptions. . . . . . . . . . . . 86

5.21 Quadrature variances vs. pump ratio under ideal assumpations. . . . . . . . . . . . 86

5.22 Full-model spectral fits. . . . . . . . . . . . . . . . . . . . . . . . . . . 88

5.23 Comparison of full analysis to simple fit results. . . . . . . . . . . . . . . . . 89

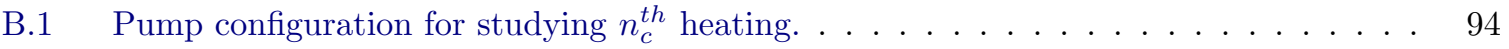




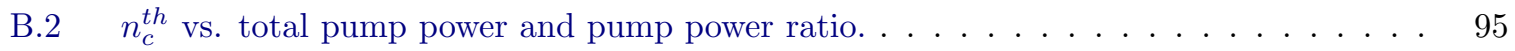

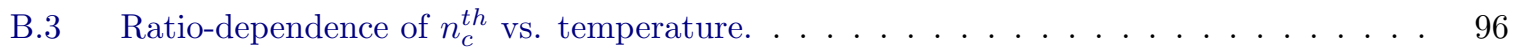

B.4 $n_{c}^{t h}$ heating map for two red-detuned tones $\ldots \ldots \ldots \ldots \ldots$

B.5 Frequency configuration for 3 omega cancellation. . . . . . . . . . . . . 97

B.6 Squeezing spectra at different 3-omega tone phases. . . . . . . . . . . . . . . 97

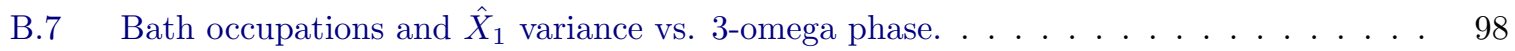




\section{Chapter 1}

\section{Introduction}

In popular science, quantum mechanics is seen mainly as a source of non-intuitive, "spooky" phenomena that we can exploit to produce new technologies. Lasers, transistors, and scanning tunneling microscopes are all touted as applications of quantum mechanics. Superconductors are commonly found in MRI magnets and SQUID magnetometers, and have been used in maglev train prototypes. Quantum key distribution systems that use either quantum indeterminacy or entanglement to securely share keys between multiple parties are already available from several companies. Quantum computers, which rely on superposition and entanglement to perform calculations at faster speeds than their classical counterparts, are seen as the technology of the future.

Less attention is given to the limits and constraints placed by quantum mechanics. Built into the fundamental assumption that quantum states can be represented as a linear vector space is the Schwarz inequality: for any two vectors $p$ and $q$ in the linear vector space with a defined inner product $\langle$,$\rangle ,$

$$
\langle p, p\rangle\langle q, q\rangle \geq|\langle p, q\rangle|^{2}
$$

If $p$ and $q$ do not commute, then the right-hand side of the above equation will be greater than 0 . This leads to the familiar Heisenberg uncertainty principle for the position, $\hat{x}$, and momentum, $\hat{p}$, of a particle:

$$
\Delta \hat{x}^{2} \Delta \hat{p}^{2} \geq|\langle[\hat{x}, \hat{p}]\rangle|^{2} / 4=\hbar^{2} / 4
$$

Through the uncertainty principle, it is impossible to simultaneously localize a particle's state in both position and momentum space. The uncertainty principle also leads to the ground state of a harmonic oscillator having a non-zero energy. As the harmonic oscillator must have $\hat{x}^{2} \geq \Delta \hat{x}^{2}$ and 
$\hat{p}^{2} \geq \Delta \hat{p}^{2}$, the oscillator's energy must satisfy

$$
E \geq \frac{\Delta \hat{p}^{2}}{2 m}+\frac{1}{2} m \omega_{m}^{2} \Delta \hat{x}^{2}
$$

Since $\Delta \hat{p}^{2} \geq \hbar^{2} / 4 \Delta \hat{x}^{2}$

$$
E \geq \frac{\hbar^{2}}{8 m \Delta \hat{x}^{2}}+\frac{1}{2} m \omega_{m}^{2} \Delta \hat{x}^{2}
$$

which is minimized when $\Delta \hat{x}=\sqrt{\hbar / 2 m \omega_{m}}$ and $E=\frac{1}{2} \hbar \omega_{m}$. Thus, as a result of the quantum, wavelike nature of the physical world, a harmonic oscillator can never be completely at rest. Even in the quantum ground state, its position will always have fluctuations with variance $\Delta \hat{x}_{z p}^{2}=\hbar /\left(2 m \omega_{m}\right)$, called the zero-point fluctuations.

These zero-point fluctuations place limits on continuous position measurements of mechanical resonators. Such measurements are often performed in the context of force detection, and micro-scale mechanical resonators have been used to sense electric forces due to the presence of charges [12] and magnetic forces from single spins [46]. They are also widely-used as accelerometers and gyroscopes [65]. On a much larger scale, mechanical resonators are fundamental to many gravitational wave detectors. Bar resonator detectors, from the original Weber bars [59] to the more-recent Nautilus [6] and AURIGA [67] projects, detect displacements of a 1000-kg-scale "antenna" for gravitational waves. Interferometric detectors like GEO [63], LIGO [2], and VIRGO [3] essentially measure the position of the 10-kg-scale mirrors at the ends of their interferometer arms. For all these systems, zero-point fluctuations place a lower bound on the minimum measurable displacement of the mechanical objects.

In addition to the intrinsic zero-point motion of a mechanical object, the act of measuring position must itself add noise. The quantum limits on continuous position measurements of mechanical resonators were first studied theoretically in the context of interferometers [7, 9]. To illustrate theses limits, it is thus useful to consider the end mirror of a Fabry-Pérot cavity in one arm of a Michelson interferometer (Fig. 1.1a). When the cavity is driven at its resonance frequency, $\omega_{c}$, the motion of the mechanics modulates the phase of the laser drive, and thus the mechanical position can be measured from the resulting phase shift. The laser itself has shot noise, which leads to uncertainty in the measured phase. This "imprecision" noise is evident as a white noise floor in the position noise spectral density, $S_{x x}(\omega)$. The zero-point motion of the mechanics contributes a Lorentzian centered at the mechanical resonance frequency, $\omega_{m}$, and with a linewidth equal to the intrinsic mechanical damping rate, $\gamma_{m}$, to $S_{x x}$. As the laser power, $P$, is increased, the measurement uncertainty is decreased, and thus the imprecision decreases. However, the shot noise of the laser begins to drive the mechanical motion, leading to a larger Lorentzian response centered at $\omega_{m}$. The 
contributions of imprecision, back-action, and zero-point fluctuations at different laser powers are shown in Fig. 1.1b\&c. There exists an optimum pump power for which the total noise is minimized; this point is referred to as the "standard quantum limit" (SQL). At the standard quantum limit power, the imprecision noise and back-action noise at $\omega_{m}$ each contribute half the zero-point noise, leading to a total position noise twice the zero-point fluctuations. While we have considered the limits of measurement for the specific case of a mirror in an optical cavity, the SQL represents the minimum noise of any continuous, weak measurement of position. For further discussion, [13] includes an indepth derivation of the quantum limits on position measurement.

In practice, most position measurement systems have noise contributions above the limits presented here. For example, a linear amplifier adds to the measurement imprecision even in the quantum limit, and classical noise in the cavity can lead to additional back-action. Since I joined the Schwab group in 2009, much work has been done in the field of optomechanics to simply perform measurements at the level of these limits. Several groups have performed measurements of imprecision "below the SQL" - that is, at high-enough powers that the imprecision noise floor contributes less than $S_{x x}^{0}\left(\omega_{m}\right) / 2[55,4,61]$. Other groups have observed contributions from the shot-noise backaction (also referred to as "radiation pressure shot noise") $[43,51]$. So far, no group has been able to perform a measurement with both imprecision and back-action at the standard quantum limit.

In the derivations and experiments discussed so far, the measurements have been insensitive to the phase of the mechanical motion. The position of the mechanical resonator can be broken down into two orthogonal motional quadratures, $\hat{X}_{1}$ and $\hat{X}_{2}$, which are related to the position operator by $\hat{x}=\sqrt{2} x_{z p}\left(\hat{X}_{1} \cos \omega_{m} t+\hat{X}_{2} \sin \omega_{m} t\right)$. These non-commuting quadrature operators define a set of axes that rotate through position-momentum space at a frequency $\omega_{m}$. If we are only interesting in measuring one of these quadratures, it is possible to avoid the imprecision and back-action noise at the expense of giving up information or adding noise to the orthogonal quadrature. Over the past several years, the shot-noise imprecision has been avoided by using quadrature-squeezed light for the laser drive [26, 1], and the quantum back-action has been evaded in single-quadrature measurements via parametric modulation of the optomechanical coupling [51]. The next logical step is to avoid the zero-point fluctuations in a single quadrature by squeezing the mechanical motion.

In the quantum ground state, a mechanical resonator has position fluctuations divided equally between $\hat{X}_{1}$ and $\hat{X}_{2}$. The ground-state fluctuations minimize the uncertainty relation given by the quadratures' non-zero commutator:

$$
\begin{array}{r}
\left\langle\Delta \hat{X}_{1}^{2}\right\rangle\left\langle\Delta \hat{X}_{2}^{2}\right\rangle \geq \frac{1}{4}\left|\left\langle\left[\hat{X}_{1}, \hat{X}_{2}\right]\right\rangle\right|^{2}=1 / 4 \\
\left\langle\Delta \hat{X}_{1}^{2}\right\rangle_{Z P}=\left\langle\Delta \hat{X}_{2}^{2}\right\rangle_{Z P}=1 / 2 .
\end{array}
$$

Given this uncertainty relation, it is, in principle, possible to squeeze the zero-point noise such that 
a)

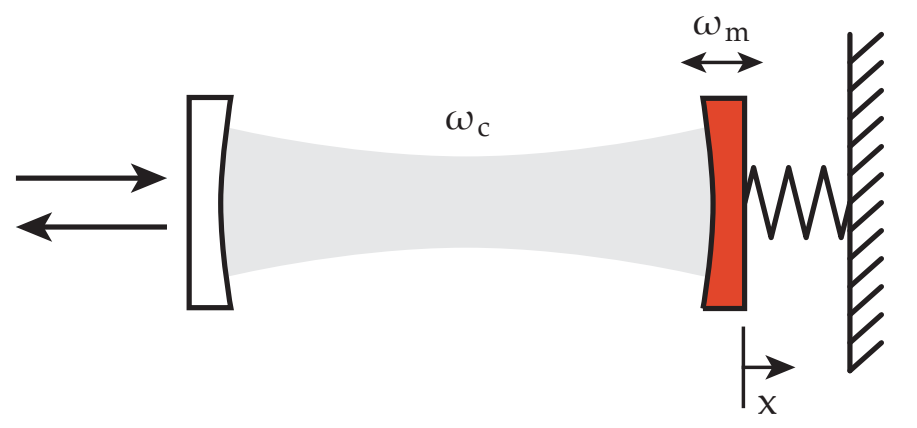

b)

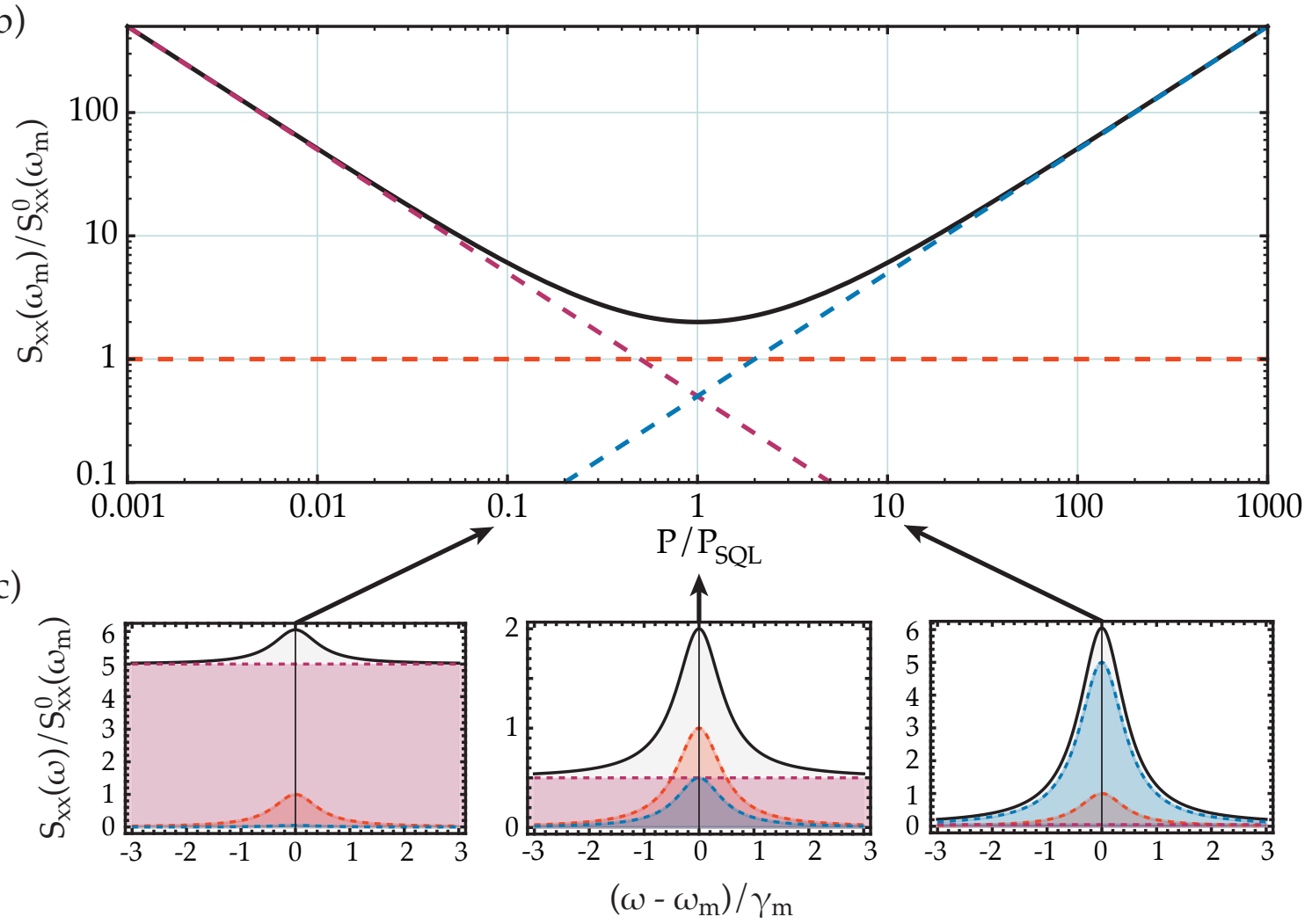

Figure 1.1: Quantum noise limits on continuous position measurement. a) Example of a continuous position measurement scheme. One mirror (red) of a Fabry-Pérot cavity with frequency $\omega_{c}$ has a mechanical resonance with frequency $\omega_{m}$ and loss rate $\gamma_{m}$. The position of the mirror changes the length of the cavity, shifting the phase of a laser drive at $\omega_{c}$. b) Total position noise spectral density measured at the mechanical frequency, in units of the corresponding zero-point noise. The zero-point fluctuations (dashed red line) are constant for all applied powers. The imprecision (dashed purple line) decreases with applied power, while the back-action (dashed blue line) increases. The total noise is shown in black. At $P_{S Q L}$, the back-action and imprecision are equal, and each contribute half of the zero-point fluctuations. c) Noise spectral density vs. frequency for $P / P_{S Q L}=0.1$ (left), 1.0 (center), and 10 (right). Imprecision noise is shown in purple, back-action noise is shown in blue, and the zero-point fluctuations are shown in red. The total noise spectral density is shown in black. For low powers, the imprecision dominates the total noise, while at high powers, the back-action dominates. 
fluctuations in one quadrature are reduced below the zero-point level at the expense of increasing noise in the orthogonal quadrature. More generally, other non-commuting observable pairs can be squeezed, and quantum squeezed states ${ }^{1}$ have been created and detected in such varied systems as optical [49] and microwave [66] modes, the motion of trapped ions [37], and spin states in an ensemble of cold atoms [24]. Transient quantum squeezing has also been created and observed in the motion of molecular nuclei [16] and of terahertz-frequency phonons in an atomic lattice on picosecond timescales [21]. While [37], [16], and [21] all produce quantum squeezed states of motion, when it comes to potential applications, they do not have the same advantages as the steady-state squeezing of the engineered, high-Q mechanical resonator that we deal with in this work. Moreover, a mesoscopic membrane is, in many ways, a more "classical" object than a collection of ions or phonons, and quantum manipulation of larger, more macroscopic systems is a current goal of experimental physics.

While a squeezed thermal state always has a positive Wigner function, when the fluctuations in one quadrature are reduced below the zero-point level, the squeezed state no longer has a wellbehaved P-representation [29] - that is, it cannot be represented as an incoherent mixture of coherent states, which are often referred to as the "most classical" of quantum states. For this reason, a quantum squeezed state is considered a non-classical state [22]. Much effort has gone into producing and studying non-classical behavior in larger and larger systems, and recent progress in the field of optoand electromechanics has resulted in the generation of mechanical Fock states [39], entanglement [40], and observations of quantum sideband asymmetry [32, 38]. Quantum squeezing in a micron-scale mechanical resonator is an important addition to this short list.

A major challenge for quantum squeezing of a radio-frequency mechanical mode is that, even at a temperature of $10 \mathrm{mK}$, the thermal occupation and corresponding position fluctuations are far larger than the quantum zero-point fluctuations: $\Delta \hat{x}^{2} \sim 100 \cdot \Delta \hat{x}_{z p}^{2}$ for a $4 \mathrm{MHz}$ resonator; quantum squeezing can only be accomplished by first overcoming this large thermal contribution. In contrast, optical modes are found in the quantum ground state at room temperature. Squeezing of mechanical fluctuations was first demonstrated far outside the quantum regime by parametrically modulating the mechanical spring constant [47]. Since parametric methods are limited to $3 \mathrm{~dB}$ of steady-state squeezing, the occupation factor of the mechanical mode must be well below one phonon to achieve squeezing below the zero-point fluctuations. While sideband cooling has led to occupations of less than one phonon in recent years [56, 10], nonlinearities and heating tend to prevent the phonon occupation from dropping far below 1 , making quantum squeezing via parametric techniques difficult. There are many theoretical proposals for surpassing the $3 \mathrm{~dB}$ limit to produce quantum squeezing [44, 48, 14, 68, 27, 34, 54, 57, 23, 5, 33], and improvement over the $3 \mathrm{~dB}$ limit

\footnotetext{
${ }^{1}$ Throughout this thesis, I will refer to "quantum" squeezed states as states where noise in one quadrature (or one non-commuting observable) is reduced below its zero-point value. Minimization of the uncertainty relation is not required in this definition.
} 


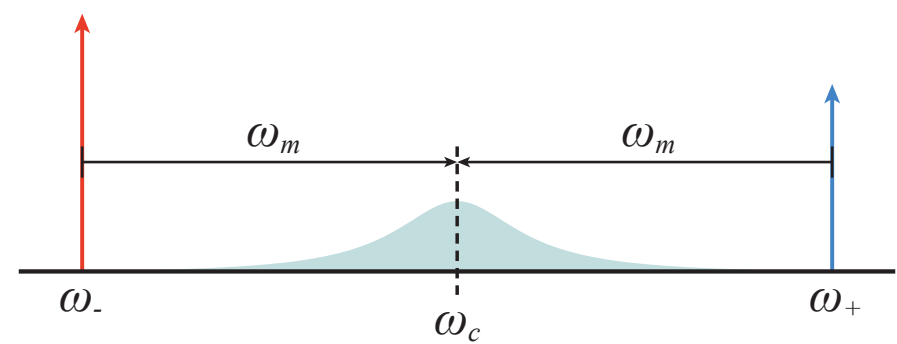

Figure 1.2: Squeezing drive schematic, showing the relative frequencies and amplitudes of the redand blue-detuned pump tones.

has been realized experimentally with modified parametric techniques $[53,58,42]$. Squeezing below the zero-point fluctuations, however, has yet to be achieved.

In the experiments described in this dissertation and in [64], we implement a reservoir-engineering scheme proposed by Kronwald, Marquardt, and Clerk [30]. This method is closely related to the approach of Cirac et. al. [11] that was recently used to produce quantum squeezed states in the motion of trapped ions [28]. Squeezing via reservoir engineering has advantages over other methods, as it creates a system in which the mechanics relaxes into a steady-state squeezed state without the fast measurements and control necessary for feedback. The scheme consists of applying two pump tones to an optical or microwave cavity parametrically-coupled to a mechanical resonator. The pumps are detuned from the cavity frequency, $\omega_{c}$, by the mechanical frequency, $\pm \omega_{m}$, and the red-detuned (-) pump has a greater amplitude than the blue-detuned $(+)$ pump (Fig. 1.2). This is a similar set-up to one used for a back-action-evading (BAE) measurement of a single quadrature [8], but with excess red power. As the blue/red pump occupation ratio, $n_{p}^{+} / n_{p}^{-}$, goes to 0 , the pump configuration becomes a single, red-detuned drive, as in sideband cooling [35]. In this limit, the fluctuations of both quadratures are damped and cooled, but the final noise in each quadrature is limited by the zero-point noise of the cavity and drive. As the ratio goes to 1 , the pump configuration becomes the balanced drives of BAE. In this limit, all back-action noise is added to the $\hat{X}_{2}$ quadrature, leaving the $\hat{X}_{1}$ quadrature unperturbed by the measurement, and neither quadrature is cooled. In between these limits, both quadratures are damped by the excess red power, while the backaction noise added to $\hat{X}_{1}$ is less than the zero-point noise associated with the total damping. By optimizing the pump ratio between these two limits, it is possible to produce arbitrarily-large amounts of sub-zero-point squeezing (i.e., $>3 \mathrm{~dB}$ ) if the coupling between the mechanics and the squeezed reservoir sufficiently dominates the mechanical dissipation rate.

While I worked on many projects throughout my tenure in the Schwab group, including superconducting parametric amplifiers, graphene bolometers, and back-action evading measurements, 
I have chosen to focus this dissertation on a thorough description of our squeezing experiments. The measurements presented here represent the first evidence of quantum squeezing in a mechanical resonator. They demonstrate the effectiveness of the Kronwald, Marquardt, and Clerk (KMC) squeezing method, even in a system where the mechanics start out with 100 times the zero-point fluctuations, and where both the cavity and mechanical baths are subject to power-dependent heating.

This dissertation is laid out as follows: In Chapter 2, I derive the theoretical background necessary for understanding our calibrations and measurements. In Chapter 3, I introduce our measurement set-up and calibration procedures. In Chapters 4 and 5, I present measurements of squeezing for two devices, which I will refer to as Device 1 (D1) and Device 2 (D2). For D1, we perform back-action evading measurements of a squeezed state, showing the full phase dependence of the mechanical motion. For D2, we fit the output noise spectrum of photons exiting the cavity, and find evidence that the mechanics are squeezed such that the $\hat{X}_{1}$ quadrature has fluctuations at $0.80 \pm 0.03$ times the zero-point level. Appendix A includes definitions of variables and functions used throughout this dissertation, and Appendix B includes a characterization of the heating behavior we see in our devices. 


\section{Chapter 2}

\section{Theory}

Here, I derive the equations governing our system using input-output theory. Standard references for this approach include [19] and [20]. The main objectives of this derivation are to

- Find the quadrature variances produced when our electromechanical device is driven with two tones at $\omega_{ \pm}=\omega_{c} \pm \omega_{m}$ when both the mechanics and cavity are coupled to thermal baths with non-zero temperatures (Section 2.4).

- Derive measureable quantities for our system in the presence of the squeezing tones, such as the cavity transmission (Section 2.5) and the noise spectrum (Section 2.6).

- Find the effects of imperfect detuning (i.e., $\omega_{ \pm} \neq \omega_{c} \pm \omega_{m}$ ) on both the amount of squeezing (Section 2.4.4) and on measurable quantities (Sections 2.5 and 2.6).

- Provide the theoretical background necessary for understanding the BAE probe measurements of Chapter 4 (Section 2.7).

- Derive equations necessary for understanding our typical calibrations and device characterization, including our two-tone thermal calibration (Section 2.6.2) and single-tone linewidth broadening (Section 2.8.2) and cooling (Section 2.8.1).

These derivations represent a combination of results already published by other authors [30, 13, 14, 60, 51, 35, 45], unpublished notes and comments from within our group (Chan U Lei) or from our theory collaborators (Andreas Kronwald, Anja Metelmann, and Aashish Clerk), and my own calculations. The main results discussed here that are not included in the KMC squeezing paper are the inclusion of a non-zero thermal cavity occupation and of the effects of imperfect pump alignment. The effects of imbalanced pump power on BAE measurements are also, to my knowledge, unpublished. 


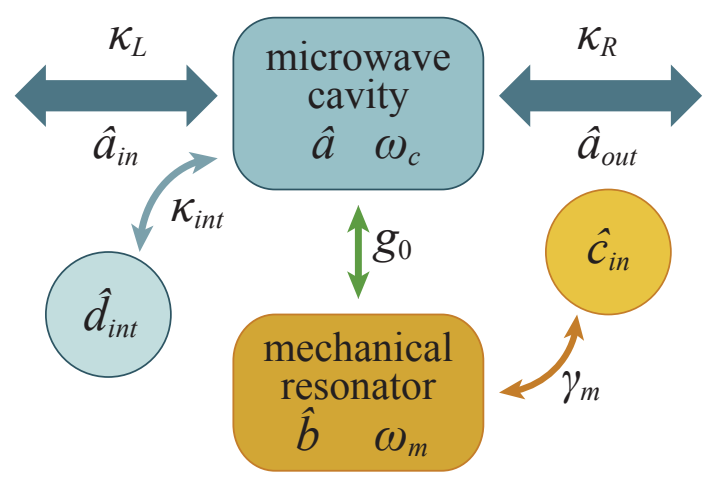

Figure 2.1: Cartoon of the mechanical and cavity resonators with definitions of the coupling to input, output, and internal baths.

\subsection{Setting up the system}

\subsubsection{Parameters of the system}

We begin by considering a microwave cavity with resonant frequency $\omega_{c}$ and a mechanical resonator with frequency $\omega_{m}$ and effective mass $m$, defining the photon and phonon annihilation operators as $\hat{a}$ and $\hat{b}$, respectively. The position of the mechanics is then given by

$$
\hat{x}=x_{z p}\left(\hat{b}+\hat{b}^{\dagger}\right)
$$

where $x_{z p}=\sqrt{\hbar /\left(2 \omega_{m} m\right)}$ is the rms amplitude of the zero-point fluctuations of the mechanical oscillator in its quantum ground state. As the frequency of the cavity depends on the position of the mechanics, the two are coupled together with a coupling constant $g=d \omega_{c} / d x$. We define the single-photon coupling rate, $g_{0}=g x_{z p}$, as the cavity frequency shift for a displacement of $x_{z p}$.

In order to send photons into the cavity and read out photons from the cavity, we must couple it to a transmission line. In our system, the microwave cavity couples to a left (input) and a right (output) port with coupling rates $\kappa_{L}$ and $\kappa_{R}$, respectively. Moreover, neither the mechanics nor the cavity are perfectly isolated - they both have internal losses that provide coupling to external baths. The internal loss rate for the cavity is $\kappa_{i n t}$, and the intrinsic loss rate for the mechanics is $\gamma_{m}$. We further define the total cavity loss rate as $\kappa=\kappa_{L}+\kappa_{R}+\kappa_{\text {int }}$. This system is depicted in Fig. 2.1.

\subsubsection{Input noise operators}

Coupling to the environment not only leads to photons and phonons leaving the system, but also allows noise to enter the system. We define the input phonon noise annihilation operator to be $\hat{c}_{i n}$, and the photon noise annihilation operators for each port to be $\hat{d}_{i n, i}$, where $i=L, R$, int. These 
operators all obey the canonical commutation relations, and have the following expectation values:

$$
\begin{aligned}
\left\langle\hat{d}_{i n, i}^{\dagger}(t) \hat{d}_{i n, j}\left(t^{\prime}\right)\right\rangle & =n_{c, i}^{t h} \delta_{i j} \delta\left(t-t^{\prime}\right) \\
\left\langle\hat{d}_{i n, i}(t) \hat{d}_{i n, j}^{\dagger}\left(t^{\prime}\right)\right\rangle & =\left(n_{c, i}^{t h}+1\right) \delta_{i j} \delta\left(t-t^{\prime}\right) \\
\left\langle\hat{c}_{i n}^{\dagger}(t) \hat{c}_{i n}\left(t^{\prime}\right)\right\rangle & =n_{m}^{t h} \delta\left(t-t^{\prime}\right) \\
\left\langle\hat{c}_{i n}(t) \hat{c}_{i n}^{\dagger}\left(t^{\prime}\right)\right\rangle & =\left(n_{m}^{t h}+1\right) \delta\left(t-t^{\prime}\right)
\end{aligned}
$$

Here, $n_{i}^{t h}$ represents the Bose occupancy of a given bath at temperature $T_{i}: n_{i}^{t h}=\left(e^{\hbar \omega_{i} / k_{B} T_{i}}-1\right)^{-1}$. At 0 temperature, $n_{i}^{t h}=0$. At $10 \mathrm{mK}$, we expect $n_{c, i}^{t h}$, the microwave occupations, to be effectively 0 , and $n_{m}^{\text {th }}$ to be between 10 and 100 for our MHz-frequency devices. By applying strong driving tones, however, we can substantially heat these baths; in general, $n_{m}^{\text {th }}$ and $n_{c, \text { int }}^{\text {th }}$ will be dependent on the applied power.

To simplify future calculations, we can define

$$
\hat{d}_{i n}=\sum_{i=L, R, i n t} \frac{\sqrt{\kappa_{i}}}{\sqrt{\kappa}} \hat{d}_{i n, i}
$$

so that $\hat{d}_{i n}$ has the following expectation values:

$$
\begin{aligned}
& \left\langle\hat{d}_{i n}^{\dagger}(t) \hat{d}_{i n}\left(t^{\prime}\right)\right\rangle=\sum_{i} \frac{\kappa_{i}}{\kappa} n_{c, i}^{t h} \delta\left(t-t^{\prime}\right) \equiv n_{c}^{t h} \delta\left(t-t^{\prime}\right) \\
& \left\langle\hat{d}_{i n}(t) \hat{d}_{i n}^{\dagger}\left(t^{\prime}\right)\right\rangle=\sum_{i} \frac{\kappa_{i}}{\kappa}\left(n_{c, i}^{t h}+1\right) \delta\left(t-t^{\prime}\right)=\left(n_{c}^{t h}+1\right) \delta\left(t-t^{\prime}\right) .
\end{aligned}
$$

Here, we have defined $n_{c}^{\text {th }}$ as the average thermal cavity occupation from the external and internal baths, weighted by their coupling rates.

It is also useful to consider the Fourier transforms of these operators. For a general operator $\hat{\xi}$, we define the Fourier transforms as:

$$
\begin{array}{ll}
\hat{\xi}[\omega]=\int_{-\infty}^{\infty} d t e^{i \omega t} \hat{\xi}(t) & \hat{\xi}^{\dagger}[\omega]=\int_{-\infty}^{\infty} d t e^{i \omega t} \hat{\xi}^{\dagger}(t)=[\hat{\xi}[-\omega]]^{\dagger} \\
\hat{\xi}(t)=\frac{1}{2 \pi} \int_{-\infty}^{\infty} d \omega e^{-i \omega t} \hat{\xi}[\omega] & \hat{\xi}^{\dagger}(t)=\frac{1}{2 \pi} \int_{-\infty}^{\infty} d \omega e^{-i \omega t} \hat{\xi}^{\dagger}[\omega] .
\end{array}
$$




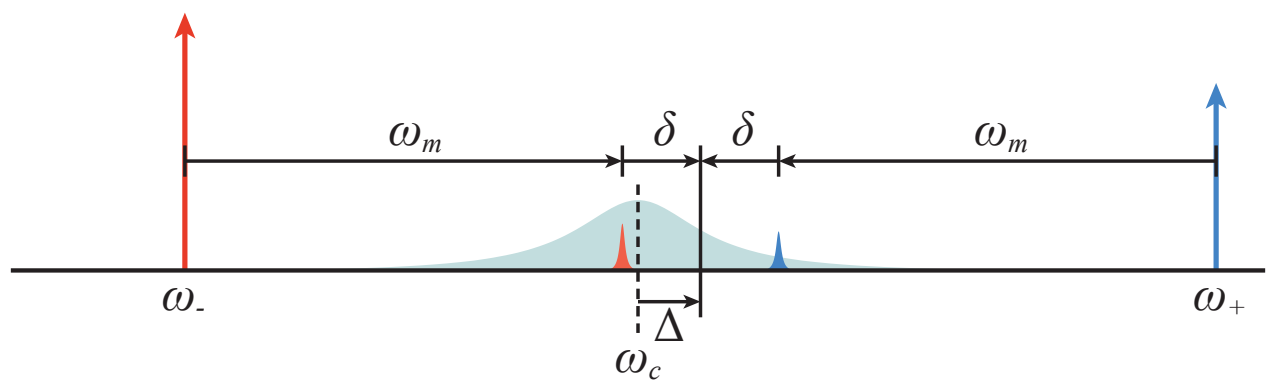

Figure 2.2: Schematic of two-tone pump configuration to show relative frequencies. Detunings are exaggerated for clarity.

In frequency space, Eq. 2.2 then becomes

$$
\begin{aligned}
\left\langle\hat{d}_{i n}^{\dagger}[\omega] \hat{d}_{i n}\left[\omega^{\prime}\right]\right\rangle & =2 \pi n_{c}^{t h} \delta\left(\omega+\omega^{\prime}\right) \\
\left\langle\hat{d}_{i n}[\omega] \hat{d}_{i n}^{\dagger}\left[\omega^{\prime}\right]\right\rangle & =2 \pi\left(n_{c}^{t h}+1\right) \delta\left(\omega+\omega^{\prime}\right) \\
\left\langle\hat{c}_{i n}^{\dagger}[\omega] \hat{c}_{i n}\left[\omega^{\prime}\right]\right\rangle & =2 \pi n_{m}^{t h} \delta\left(\omega+\omega^{\prime}\right) \\
\left\langle\hat{c}_{i n}[\omega] \hat{c}_{i n}^{\dagger}\left[\omega^{\prime}\right]\right\rangle & =2 \pi\left(n_{m}^{t h}+1\right) \delta\left(\omega+\omega^{\prime}\right) .
\end{aligned}
$$

\subsubsection{Pump configuration}

In order to implement the squeezing scheme, we drive the system with two microwave tones, one reddetuned by $\omega_{m}$ from $\omega_{c}$, and one blue-detuned by $\omega_{m}$ from $\omega_{c}: \omega_{ \pm}=\omega_{c} \pm \omega_{m}$. We will also consider the general case where the pumps are not perfectly aligned, such that $\omega_{ \pm}=\left(\omega_{c}+\Delta\right) \pm\left(\omega_{m}+\delta\right)$. Imperfect alignment of the pumps degrades squeezing and changes the appearance of the output spectrum. The pump configuration is illustrated in Fig. 2.2.

\subsection{Hamiltonian}

\subsubsection{Optomechanical Hamiltonian}

We begin with the standard optomechanical Hamiltonian:

$$
\hat{\mathcal{H}}=\hbar\left(\omega_{c}+\frac{\hbar g_{0}}{x_{z p}} \hat{x}\right) \hat{a}^{\dagger} \hat{a}+\hbar \omega_{m} \hat{b}^{\dagger} \hat{b}
$$

This Hamiltonian consists of the bare Hamiltonian for two oscillators,

$$
\hat{\mathcal{H}}_{0}=\hbar \omega_{c} \hat{a}^{\dagger} \hat{a}+\hbar \omega_{m} \hat{b}^{\dagger} \hat{b}
$$


and an interaction term due to the dependence of the cavity frequency on the position of the mechanics, $\hat{x}$ :

$$
\begin{aligned}
\hat{\mathcal{H}}_{i n t} & =\frac{\hbar g_{0}}{x_{z p}} \hat{x} \hat{a}^{\dagger} \hat{a} \\
& =\hbar g_{0}\left(\hat{b}+\hat{b}^{\dagger}\right) \hat{a}^{\dagger} \hat{a} .
\end{aligned}
$$

Applying standard input-output theory to the Heisenberg equations of motion, we can then arrive at the Langevin equations of motion for our operators:

$$
\begin{aligned}
& \dot{\hat{a}}=\frac{i}{\hbar}[\hat{\mathcal{H}}, \hat{a}]-\frac{\kappa}{2} \hat{a}-\sum_{i=L, R, i n t} \sqrt{\kappa_{i}} \hat{a}_{i n, i} \\
& \dot{\hat{b}}=\frac{i}{\hbar}[\hat{\mathcal{H}}, \hat{b}]-\frac{\gamma_{m}}{2} \hat{b}-\sqrt{\gamma_{m}} \hat{b}_{i n} .
\end{aligned}
$$

We intend to drive the system from the left port with two large, coherent tones with frequencies $\omega_{-}$and $\omega_{+}$. Otherwise, all input operators should be small fluctuations. We can thus write

$$
\begin{gathered}
\hat{a}_{i n, L}=\bar{a}_{i n}^{-} e^{-i \omega_{-} t}+\bar{a}_{i n}^{+} e^{-i \omega_{+} t}+\hat{d}_{i n, L} \\
\hat{a}_{i n, R}=\hat{d}_{i n, R} ; \quad \hat{a}_{i n, i n t}=\hat{d}_{i n, i n t} .
\end{gathered}
$$

\subsubsection{Rotating frame}

In order to simplify our calculations, we transform into a frame rotating with respect to $\hat{\mathcal{H}}_{\delta}=$ $\hbar\left(\omega_{c}+\Delta\right) \hat{a}^{\dagger} \hat{a}+\hbar\left(\omega_{m}+\delta\right) \hat{b}^{\dagger} \hat{b}$ by applying the rotation transformation $\hat{U}=e^{i \hat{\mathcal{H}}_{\delta} t / \hbar}$ :

$$
\hat{\mathcal{H}}^{\prime}=\hat{U} \hat{\mathcal{H}} \hat{U}^{\dagger}-\hat{\mathcal{H}}_{\delta}
$$

Since

$$
\begin{aligned}
\hat{U} \hat{a} \hat{U}^{\dagger} & =e^{-i\left(\omega_{c}+\Delta\right) t} \hat{a} \\
\hat{U} \hat{b} \hat{U}^{\dagger} & =e^{-i\left(\omega_{m}+\delta\right) t} \hat{b},
\end{aligned}
$$

the Hamiltonian in the rotated frame then becomes

$$
\hat{\mathcal{H}}^{\prime}=-\hbar \Delta \hat{a}^{\dagger} \hat{a}-\hbar \delta \hat{b}^{\dagger} \hat{b}+\hbar g_{0} \hat{a}^{\dagger} \hat{a}\left(e^{-i\left(\omega_{m}+\delta\right) t} \hat{b}+e^{i\left(\omega_{m}+\delta\right) t} \hat{b}^{\dagger}\right)
$$


If we transform all the input operators into the same frame, the explicit time dependence of $\hat{a}_{i n, L}$ becomes:

$$
\hat{a}_{i n, L}=\bar{a}_{i n}^{-} e^{i\left(\omega_{m}+\delta\right) t}+\bar{a}_{i n}^{+} e^{-i\left(\omega_{m}+\delta\right) t}+\hat{d}_{i n, L}
$$

We then obtain the following equations of motion from Eq. 2.11 in the rotating frame:

$$
\begin{gathered}
\dot{\hat{a}}=i \Delta \hat{a}-i g_{0} \hat{a}\left(e^{-i\left(\omega_{m}+\delta\right) t} \hat{b}+e^{i\left(\omega_{m}+\delta\right) t} \hat{b}^{\dagger}\right)-\frac{\kappa}{2} \hat{a} \\
\quad-\sqrt{\kappa_{L}}\left(\bar{a}_{i n}^{-} e^{i\left(\omega_{m}+\delta\right) t}+\bar{a}_{i n}^{+} e^{-i\left(\omega_{m}+\delta\right) t}\right)-\sqrt{\kappa} \hat{d}_{i n} \\
\dot{\hat{b}}=i \delta \hat{b}-i g_{0} \hat{a}^{\dagger} \hat{a} e^{i\left(\omega_{m}+\delta\right) t}-\frac{\gamma_{m}}{2} \hat{b}-\sqrt{\gamma_{m}} \hat{b}_{i n} .
\end{gathered}
$$

\subsection{Solving the equations of motion}

\subsubsection{Classical solution}

To linearize the equations of motion, we displace the photon and phonon operators by their classical solutions:

$$
\begin{aligned}
& \hat{a}=\bar{a}(t)+\hat{d} \\
& \hat{b}=\bar{b}(t)+\hat{c} .
\end{aligned}
$$

If we neglect the quantum operators, Eq. 2.20 gives us a system of equations for the classical amplitudes:

$$
\begin{aligned}
& \dot{\bar{a}}=i \Delta \bar{a}-\frac{\kappa}{2} \bar{a}-\sqrt{\kappa_{L}}\left(\bar{a}_{i n}^{-} e^{i\left(\omega_{m}+\delta\right) t}+\bar{a}_{i n}^{+} e^{-i\left(\omega_{m}+\delta\right) t}\right) \\
& \dot{\bar{b}}=i \delta \bar{b}-i g_{0}|\bar{a}|^{2} e^{i\left(\omega_{m}+\delta\right) t}-\frac{\gamma_{m}}{2} \bar{b} .
\end{aligned}
$$

Here, we have neglected the term $-i g_{0} \hat{a}\left(e^{-i\left(\omega_{m}+\delta\right) t} \bar{b}+e^{i\left(\omega_{m}+\delta\right) t} \bar{b}^{*}\right)$ in the equation of motion for $\bar{a}$. This term creates a shift in the cavity frequency that depends on the classical value for the position. From now on, we will assume that $\omega_{c}$ refers to this shifted frequency, and that $\Delta$ is defined with respect to the new $\omega_{c}$. We can then solve for the classical solutions:

$$
\begin{aligned}
& \bar{a}=\bar{a}_{-} e^{i\left(\omega_{m}+\delta\right) t}+\bar{a}_{+} e^{-i\left(\omega_{m}+\delta\right) t} \\
& \bar{b}=-i g_{0}\left(\frac{\bar{a}_{-}^{2}+\bar{a}_{+}^{2}}{\frac{\gamma_{m}}{2}+i \omega_{m}}+\frac{\bar{a}_{-} \bar{a}_{+}}{\frac{\gamma_{m}}{2}-i \omega_{m}} e^{-2 i \omega_{m} t}+\frac{\bar{a}_{-} \bar{a}_{+}}{\frac{\gamma_{m}}{2}+6 i \omega_{m}} e^{2 i \omega_{m} t}\right) e^{i\left(\omega_{m}+\delta\right) t},
\end{aligned}
$$

where

$$
\bar{a}_{-}=\frac{-\sqrt{\kappa_{L}}}{\frac{\kappa}{2}+i\left(\omega_{m}+\delta-\Delta\right)} \bar{a}_{i n}^{-} ; \quad \bar{a}_{+}=\frac{-\sqrt{\kappa_{L}}}{\frac{\kappa}{2}+i\left(-\omega_{m}-\delta-\Delta\right)} \bar{a}_{i n}^{+}
$$


Without loss of generality, we can choose the phases of the input amplitudes such that $\bar{a}_{ \pm}$are real. The classical amplitudes are related to the intracavity pump photon numbers, $n_{p}^{-}$and $n_{p}^{+}$, by $n_{p}^{ \pm}=\sqrt{\bar{a}_{ \pm}}$.

With the classical terms removed, we obtain the linearized equations of motion for the quantum noise operators:

$$
\begin{aligned}
& \dot{\hat{d}}=i \Delta \hat{d}-i g_{0} \bar{a}\left(e^{-i\left(\omega_{m}+\delta\right) t} \hat{c}+e^{i\left(\omega_{m}+\delta\right) t} \hat{c}^{\dagger}\right)-\frac{\kappa}{2} \hat{d}-\sqrt{k c} \hat{d}_{i n} \\
& \dot{\hat{c}}=i \delta \hat{c}-i g_{0}\left(\bar{a}^{*} \hat{d}+\bar{a} \hat{d}^{\dagger}\right) e^{i\left(\omega_{m}+\delta\right) t}-\frac{\gamma_{m}}{2} \hat{c}-\sqrt{\gamma_{m}} \hat{c}_{i n}
\end{aligned}
$$

Note that we neglect the contributions from the $\hat{d}^{\dagger} \hat{d}$ term in the Hamiltonian, as the cavity noise occupation is much smaller than the intracavity pump occupation.

\subsubsection{Rotating wave approximation}

Per Eq. 2.21, $\bar{a}$ has terms at frequencies $\pm\left(\omega_{m}+\delta\right)$. When we substitute the expression for $\bar{a}$ into Eq. 2.23, we thus find that some terms are stationary with respect to our rotating frame, and some rotate at $\pm 2\left(\omega_{m}+\delta\right)$. In our sideband-resolved case $\left(\omega_{m} \gg \kappa\right)$, the contributions from the stationary terms dominate, and thus we can set the $\pm 2\left(\omega_{m}+\delta\right)$ terms equal to zero. Under this rotating wave approximation (RWA), the equations of motion become:

$$
\begin{aligned}
& \dot{\hat{d}}=i \Delta \hat{d}-i g_{0}\left(\bar{a}_{-} \hat{c}+\bar{a}_{+} \hat{c}^{\dagger}\right)-\frac{\kappa}{2} \hat{d}-\sqrt{k c} \hat{d}_{i n} \\
& \dot{\hat{c}}=i \delta \hat{c}-i g_{0}\left(\bar{a}_{-} \hat{d}+\bar{a}_{+} \hat{d}^{\dagger}\right)-\frac{\gamma_{m}}{2} \hat{c}-\sqrt{\gamma_{m}} \hat{c}_{i n}
\end{aligned}
$$

Without the rotating wave approximation, we are left with a series of equations that couple together terms rotating at $\omega=\ldots,-4\left(\omega_{m}+\delta\right),-2\left(\omega_{m}+\delta\right), 0,2\left(\omega_{m}+\delta\right), 4\left(\omega_{m}+\delta\right), \ldots$, etc. with respect to the current frame. These equations are solvable to the desired order, but the analytic solutions become unwieldy for even first-order corrections. Implementation of non-RWA corrections (also called "bad-cavity" effects) is thus done numerically. 


\subsubsection{Heisenberg-Langevin equations}

We can now use Eq. 2.24 to write down the Heisenberg-Langevin equations for all mode operators in our system:

$$
\begin{aligned}
\dot{\hat{d}} & =\left(i \Delta-\frac{\kappa}{2}\right) \hat{d}-\sqrt{\kappa} \hat{d}_{i n}-i\left[G_{-} \hat{c}+G_{+} \hat{c}^{\dagger}\right] \\
\dot{\hat{d}}^{\dagger} & =\left(-i \Delta-\frac{\kappa}{2}\right) \hat{d}^{\dagger}-\sqrt{\kappa} \hat{d}_{i n}^{\dagger}+i\left[G_{-} \hat{c}^{\dagger}+G_{+} \hat{c}\right] \\
\dot{\hat{c}} & =\left(i \delta-\frac{\gamma_{m}}{2}\right) \hat{c}-\sqrt{\gamma_{m}} \hat{c}_{i n}-i\left[G_{-} \hat{d}+G_{+} \hat{d}^{\dagger}\right] \\
\dot{\hat{c}}^{\dagger} & =\left(-i \delta-\frac{\gamma_{m}}{2}\right) \hat{c}^{\dagger}-\sqrt{\gamma_{m}} \hat{c}_{i n}^{\dagger}+i\left[G_{-} \hat{d}^{\dagger}+G_{+} \hat{d}\right],
\end{aligned}
$$

where $G_{ \pm}=g_{0} \bar{a}_{ \pm}$are the enhanced optomechanical coupling rates. We then take the Fourier transform of this system of equations to find the frequency-domain operators:

$$
\begin{aligned}
\left(\frac{\kappa}{2}-i(\omega+\Delta)\right) \hat{d}[\omega] & =-\sqrt{\kappa} \hat{d}_{i n}[\omega]-i G_{-} \hat{c}[\omega]-i G_{+} \hat{c}^{\dagger}[\omega] \\
\left(\frac{\kappa}{2}-i(\omega-\Delta)\right) \hat{d}^{\dagger}[\omega] & =-\sqrt{\kappa} \hat{d}_{i n}^{\dagger}[\omega]+i G_{-} \hat{c}^{\dagger}[\omega]+i G_{+} \hat{c}[\omega] \\
\left(\frac{\gamma_{m}}{2}-i(\omega+\delta)\right) \hat{c}[\omega] & =-\sqrt{\gamma_{m}} \hat{c}_{i n}[\omega]-i G_{-} \hat{d}[\omega]-i G_{+} \hat{d}^{\dagger}[\omega] \\
\left(\frac{\gamma_{m}}{2}-i(\omega-\delta)\right) \hat{c}^{\dagger}[\omega] & =-\sqrt{\gamma_{m}} \hat{c}_{i n}^{\dagger}[\omega]+i G_{-} \hat{d}^{\dagger}[\omega]+i G_{+} \hat{d}[\omega] .
\end{aligned}
$$

Eq. 2.26 can be written as a matrix operation of the form $\mathbf{A x}=\mathbf{x}_{i n}$ :

$$
\begin{array}{rccc}
\left(\begin{array}{cccc}
\frac{\kappa}{2}-i(\omega+\Delta) & 0 & i G_{-} & i G_{+} \\
0 & \frac{\kappa}{2}-i(\omega-\Delta) & -i G_{+} & -i G_{-} \\
i G_{-} & i G_{+} & \frac{\gamma_{m}}{2}-i(\omega+\delta) & 0 \\
-i G_{+} & -i G_{-} & 0 & \frac{\gamma_{m}}{2}-i(\omega-\delta)
\end{array}\right)\left(\begin{array}{c}
\hat{d}[\omega] \\
\hat{d}^{\dagger}[\omega] \\
\hat{c}[\omega] \\
\hat{c}^{\dagger}[\omega]
\end{array}\right) \\
=-\left(\begin{array}{c}
\sqrt{\kappa} \hat{d}_{i n}[\omega] \\
\sqrt{\kappa} \hat{d}_{i n}^{\dagger}[\omega] \\
\sqrt{\gamma_{m}} \hat{c}_{i n}[\omega] \\
\sqrt{\gamma_{m}} \hat{c}_{i n}^{\dagger}[\omega]
\end{array}\right)
\end{array}
$$

The inverse of this matrix can be found analytically, and thus the system photon and phonon operators can be found in terms of the inputs. 


\subsection{Quadrature squeezing}

\subsubsection{Quadrature operators}

For squeezing and back-action evasion, it is helpful to consider the equations of motion for the quadrature operators for the cavity and mechanics:

$$
\begin{array}{ll}
\hat{U}_{1}=\frac{1}{\sqrt{2}}\left(\hat{d}^{\dagger}+\hat{d}\right) & \hat{X}_{1}=\frac{1}{\sqrt{2}}\left(\hat{c}^{\dagger}+\hat{c}\right) \\
\hat{U}_{2}=\frac{i}{\sqrt{2}}\left(\hat{d}^{\dagger}-\hat{d}\right) & \hat{X}_{2}=\frac{i}{\sqrt{2}}\left(\hat{c}^{\dagger}-\hat{c}\right) .
\end{array}
$$

Using the input expectation values from Eq. 2.7, we can also find the expectation values for the quadrature inputs:

$$
\begin{array}{ll}
\left\langle\hat{U}_{1 i n}[\omega] \hat{U}_{1 i n}\left[\omega^{\prime}\right]\right\rangle=2 \pi\left(n_{c}^{t h}+1 / 2\right) \delta\left[\omega+\omega^{\prime}\right] & \left\langle\hat{X}_{1 i n}[\omega] \hat{X}_{1 i n}\left[\omega^{\prime}\right]\right\rangle=2 \pi\left(n_{m}^{t h}+1 / 2\right) \delta\left[\omega+\omega^{\prime}\right] \\
\left\langle\hat{U}_{1 i n}[\omega] \hat{U}_{2 i n}\left[\omega^{\prime}\right]\right\rangle=2 \pi(i / 2) \delta\left[\omega+\omega^{\prime}\right] & \left\langle\hat{X}_{1 i n}[\omega] \hat{X}_{2 i n}\left[\omega^{\prime}\right]\right\rangle=2 \pi(i / 2) \delta\left[\omega+\omega^{\prime}\right] \\
\left\langle\hat{U}_{2 i n}[\omega] \hat{U}_{2 i n}\left[\omega^{\prime}\right]\right\rangle=2 \pi\left(n_{c}^{t h}+1 / 2\right) \delta\left[\omega+\omega^{\prime}\right] & \left\langle\hat{X}_{2 i n}[\omega] \hat{X}_{2 i n}\left[\omega^{\prime}\right]\right\rangle=2 \pi\left(n_{m}^{t h}+1 / 2\right) \delta\left[\omega+\omega^{\prime}\right] \\
\left\langle\hat{U}_{2 i n}[\omega] \hat{U}_{1 i n}\left[\omega^{\prime}\right]\right\rangle=2 \pi(-i / 2) \delta\left[\omega+\omega^{\prime}\right] & \left\langle\hat{X}_{2 i n}[\omega] \hat{X}_{1 i n}\left[\omega^{\prime}\right]\right\rangle=2 \pi(-i / 2) \delta\left[\omega+\omega^{\prime}\right] .
\end{array}
$$

\subsubsection{Frequency-domain Langevin equations}

To transform the matrix equations of motion from Eq. 2.27 into this new basis, we note that we can write Eq. 2.28 in the form $\mathbf{X}=\mathbf{T} \mathbf{x}$, where $\mathbf{X}=\left\{\hat{U}_{1}, \hat{U}_{2}, \hat{X}_{1}, \hat{X}_{2}\right\}$ is the quadrature operator vector, and where

$$
\mathbf{T}=\frac{1}{\sqrt{2}}\left(\begin{array}{cccc}
1 & 1 & 0 & 0 \\
-i & i & 0 & 0 \\
0 & 0 & 1 & 1 \\
0 & 0 & -i & i
\end{array}\right)
$$


We can then evaluate $\mathbf{T A} \mathbf{T}^{-1}$ to find a system of equations for the quadrature operators:

$$
\begin{gathered}
\left(\begin{array}{cccc}
\frac{\kappa}{2}-i \omega & \Delta & 0 & -\left(G_{-}-G_{+}\right) \\
-\Delta & \frac{\kappa}{2}-i \omega & G_{-}+G_{+} & 0 \\
0 & -\left(G_{-}-G_{+}\right) & \frac{\gamma_{m}}{2}-i \omega & \delta \\
G_{-}+G_{+} & 0 & \delta & \frac{\gamma_{m}}{2}-i \omega
\end{array}\right)\left(\begin{array}{c}
\hat{U}_{1}[\omega] \\
\hat{U}_{2}[\omega] \\
\hat{X}_{1}[\omega] \\
\hat{X}_{2}[\omega]
\end{array}\right) \\
=-\left(\begin{array}{c}
\sqrt{\kappa} \hat{U}_{1 i n}[\omega] \\
\sqrt{\kappa} \hat{U}_{2 i n}[\omega] \\
\sqrt{\gamma_{m}} \hat{X}_{1 i n}[\omega] \\
\sqrt{\gamma_{m}} \hat{X}_{2 i n}[\omega]
\end{array}\right) .
\end{gathered}
$$

Inverting the matrix in Eq. 2.31 allows us to find a general expression for the quadrature operators in terms of the input quadratures of the form $\mathbf{X}=\mathbf{D} \mathbf{X}_{i n}$, where $\mathbf{D}$ is the inverted matrix. In terms of the elements of $\mathbf{D}, \hat{X}_{1}$ is then

$$
\hat{X}_{1}[\omega]=-\sqrt{\kappa} \mathbf{D}_{31}[\omega] \hat{U}_{1 i n}[\omega]-\sqrt{\kappa} \mathbf{D}_{32}[\omega] \hat{U}_{2 i n}[\omega]-\sqrt{\gamma_{m}} \mathbf{D}_{33}[\omega] \hat{X}_{1 i n}[\omega]-\sqrt{\gamma_{m}} \mathbf{D}_{34}[\omega] \hat{X}_{2 i n}[\omega]
$$

where

$$
\begin{aligned}
\mathbf{D}_{31}[\omega] & =h[\omega]^{-1}\left\{\Delta\left(G_{-}-G_{+}\right) \chi_{m}^{-1}[\omega]+\delta\left(G_{-}+G_{+}\right) \chi_{c}^{-1}[\omega]\right\} \\
\mathbf{D}_{32}[\omega] & =h[\omega]^{-1}\left\{\left(G_{-}-G_{+}\right)\left(4 \mathcal{G}^{2}+\chi_{m}^{-1}[\omega] \chi_{c}^{-1}[\omega]\right)-\left(G_{-}+G_{+}\right) \delta \Delta\right\} \\
\mathbf{D}_{33}[\omega] & =h[\omega]^{-1}\left\{\mathcal{G}^{2} \chi_{c}^{-1}[\omega]+\chi_{m}^{-1}[\omega]\left(\left(\chi_{c}^{-1}[\omega]\right)^{2}+\Delta^{2}\right)\right\} \\
\mathbf{D}_{34}[\omega] & =h[\omega]^{-1}\left\{\Delta\left(G_{-}-G_{+}\right)^{2}-\delta\left(\left(\chi_{c}^{-1}[\omega]\right)^{2}+\Delta^{2}\right)\right\} \\
h[\omega] & =\mathcal{G}^{4}+2 \mathcal{G}^{2} \chi_{c}^{-1}[\omega] \chi_{m}^{-1}[\omega]-2 \mathcal{G}_{t o t}^{2} \Delta \delta+\left(\left(\chi_{c}^{-1}[\omega]\right)^{2}+\Delta^{2}\right)\left(\left(\chi_{m}^{-1}[\omega]\right)^{2}+\delta^{2}\right) .
\end{aligned}
$$

Here, I have introduced the effective enhanced optomechanical coupling rate $\mathcal{G}^{2}=G_{-}^{2}-G_{+}^{2}$, the total enhanced optomechanical coupling rate $\mathcal{G}_{t o t}^{2}=G_{-}^{2}+G_{+}^{2}$, and the mechanical and cavity susceptibilities

$$
\begin{aligned}
\chi_{m}[\omega] & =\left(\frac{\gamma_{m}}{2}-i \omega\right)^{-1} \\
\left.\chi_{c} \omega\right] & =\left(\frac{\kappa}{2}-i \omega\right)^{-1} .
\end{aligned}
$$

Using the expression for $\hat{X}_{1}[\omega]$, we can find the $\hat{X}_{1}$ quadrature fluctuations:

$$
\left\langle\hat{X}_{1}^{2}\right\rangle=\frac{1}{(2 \pi)^{2}} \int_{-\infty}^{\infty} \int_{-\infty}^{\infty} d \omega d \omega^{\prime}\left\langle\hat{X}_{1}[\omega] \hat{X}_{1}\left[\omega^{\prime}\right]\right\rangle
$$




\subsubsection{Special case: $\Delta=\delta=0$}

When the pumps are perfectly aligned, the expression for $\hat{X}_{1}$ simplifies to:

$$
\hat{X}_{1}[\omega]=-\sqrt{\kappa} \frac{G_{-}-G_{+}}{\mathcal{G}^{2}+\chi_{m}^{-1}[\omega] \chi_{c}^{-1}[\omega]} \hat{U}_{2 i n}[\omega]-\sqrt{\gamma_{m}} \frac{\chi_{c}^{-1}[\omega]}{\mathcal{G}^{2}+\chi_{m}^{-1}[\omega] \chi_{c}^{-1}[\omega]} \hat{X}_{1 i n}[\omega] .
$$

Note that, when $G_{+}=G_{-}$, the first term is 0 and the second term simplifies to $\sqrt{\gamma_{m}} \chi_{m}[\omega] \hat{X}_{1 i n}[\omega]$, so $\hat{X}_{1}$ has no dependence on the pump tones. This is the basis of back-action evading measurements, which are discussed later in Section 2.7.

We can integrate over $\hat{X}_{1}[\omega] \hat{X}_{1}\left[\omega^{\prime}\right]$ to find $\left\langle\hat{X}_{1}^{2}\right\rangle$ :

$$
\left\langle\hat{X}_{1}^{2}\right\rangle=\frac{1}{2 \pi} \int_{-\infty}^{\infty} d \omega\left\{\kappa \frac{\left(G_{-}-G_{+}\right)^{2}}{\left|\mathcal{G}^{2}+\chi_{m}^{-1}[\omega] \chi_{c}^{-1}[\omega]\right|^{2}}\left(n_{c}^{t h}+1 / 2\right)+\gamma_{m} \frac{(\kappa / 2)^{2}+\omega^{2}}{\left|\mathcal{G}^{2}+\chi_{m}^{-1}[\omega] \chi_{c}^{-1}[\omega]\right|^{2}}\left(n_{m}^{t h}+1 / 2\right)\right\} .
$$

As long as $4 \mathcal{G} \leq\left(\kappa-\gamma_{m}\right)$, we can evaluate the integral:

$$
\left\langle\hat{X}_{1}^{2}\right\rangle=\frac{4 \kappa\left(G_{-}-G_{+}\right)^{2}}{\left(4 \mathcal{G}^{2}+\gamma_{m} \kappa\right)\left(\kappa+\gamma_{m}\right)}\left(n_{c}^{t h}+1 / 2\right)+\frac{\gamma_{m}\left(4 \mathcal{G}^{2}+\kappa\left(\kappa+\gamma_{m}\right)\right)}{\left(4 \mathcal{G}^{2}+\gamma_{m} \kappa\right)\left(\kappa+\gamma_{m}\right)}\left(n_{m}^{t h}+1 / 2\right) .
$$

Similarly, we can solve for $\left\langle\hat{X}_{2}^{2}\right\rangle$ :

$$
\left\langle\hat{X}_{2}^{2}\right\rangle=\frac{4 \kappa\left(G_{-}+G_{+}\right)^{2}}{\left(4 \mathcal{G}^{2}+\gamma_{m} \kappa\right)\left(\kappa+\gamma_{m}\right)}\left(n_{c}^{t h}+1 / 2\right)+\frac{\gamma_{m}\left(4 \mathcal{G}^{2}+\kappa\left(\kappa+\gamma_{m}\right)\right)}{\left(4 \mathcal{G}^{2}+\gamma_{m} \kappa\right)\left(\kappa+\gamma_{m}\right)}\left(n_{m}^{t h}+1 / 2\right) .
$$

Earlier, I argued that squeezing occurs because both quadratures are damped, but less backaction is added to $\hat{X}_{1}$ than is associated with the amount of net damping. It is thus helpful to rewrite the quadrature variances in terms of the fluctuations we'd expect to obtain in the presence of a red-detuned tone with strength $\mathcal{G}$ :

$$
\begin{aligned}
& \left\langle\hat{X}_{1}^{2}\right\rangle=\left\langle\hat{X}_{\text {damp }}^{2}\right\rangle-\frac{8 \kappa G_{+}\left(G_{-}-G_{+}\right)}{\left(4 \mathcal{G}^{2}+\gamma_{m} \kappa\right)\left(\kappa+\gamma_{m}\right)}\left(n_{c}^{t h}+1 / 2\right) \\
& \left\langle\hat{X}_{2}^{2}\right\rangle=\left\langle\hat{X}_{\text {damp }}^{2}\right\rangle+\frac{8 \kappa G_{+}\left(G_{-}+G_{+}\right)}{\left(4 \mathcal{G}^{2}+\gamma_{m} \kappa\right)\left(\kappa+\gamma_{m}\right)}\left(n_{c}^{t h}+1 / 2\right) \\
& \left\langle\hat{X}_{\text {damp }}^{2}\right\rangle=\left\{\frac{\gamma_{m}\left(4 \mathcal{G}^{2}+\kappa\left(\kappa+\gamma_{m}\right)\right)}{\left(4 \mathcal{G}^{2}+\gamma_{m} \kappa\right)\left(\kappa+\gamma_{m}\right)}\left(n_{m}^{t h}+1 / 2\right)+\frac{4 \kappa \mathcal{G}^{2}\left(n_{c}^{t h}+1 / 2\right)}{\left(4 \mathcal{G}^{2}+\gamma_{m} \kappa\right)\left(\kappa+\gamma_{m}\right)}\right\} .
\end{aligned}
$$

Here, $\left\langle\hat{X}_{\text {damp }}^{2}\right\rangle$ is the variance in the presence of the net damping associated with $\mathcal{G}$, which appears identically in both quadratures. The first term in $\left\langle\hat{X}_{\text {damp }}^{2}\right\rangle$ is proportional to $n_{m}^{t h}+1 / 2$ and has a prefactor that is less than 1 for all $\mathcal{G}>0$. This term represents the cooling of the mechanical occupation. The second term is proportional to $n_{c}^{t h}+1 / 2$, and thus represents the added backaction noise from the microwave field. We see that, relative to the back-action of $\frac{4 \kappa \mathcal{G}^{2}\left(n_{c}^{t h}+1 / 2\right)}{\left(4 \mathcal{G}^{2}+\gamma_{m} \kappa\right)\left(\kappa+\gamma_{m}\right)}$ 

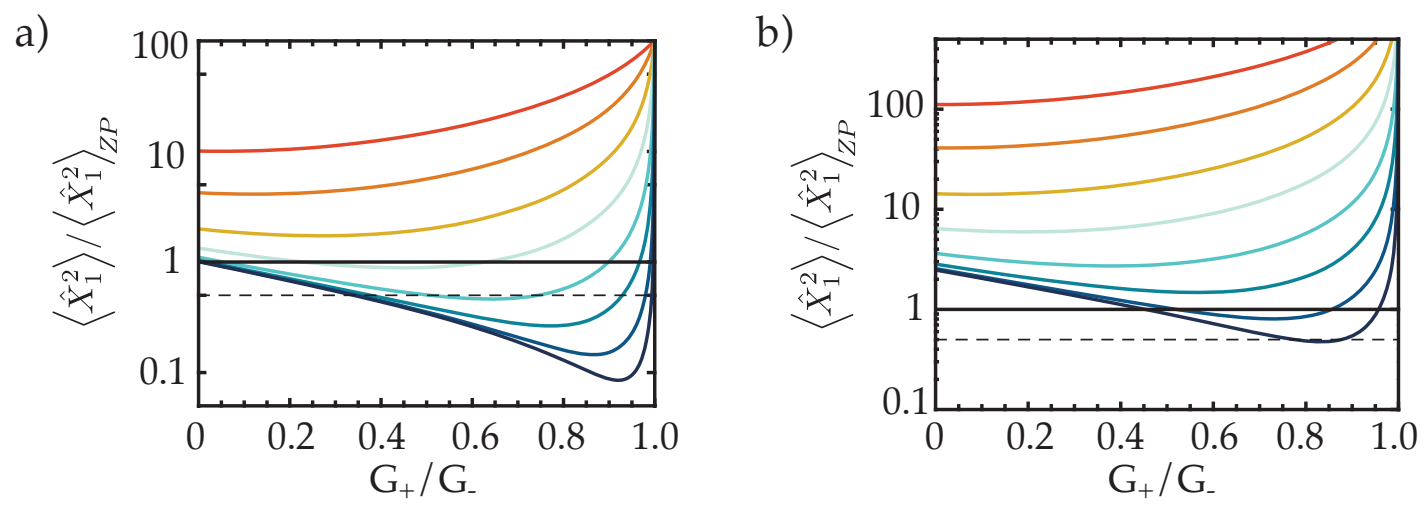

Figure 2.3: Plot of Eq. 2.38 for $\left\langle\hat{X}_{1}^{2}\right\rangle$ at different total drive strengths and drive ratios. For both plots, $\gamma_{m}=3 \times 10^{-5} \kappa$. Each curve represents a fixed total cooperativity $\left(\mathcal{C}_{t o t}=4 \mathcal{G}_{\text {tot }}^{2} / \kappa \gamma_{m}\right) . \mathcal{C}_{t o t}=$ $10,30,100,300,1000,3000,10000,30000$, with the lowest cooperativity in red and the highest in dark blue. The solid black line shows the ground state fluctuations, and the dashed black line indicates squeezing of $3 \mathrm{~dB}$ below the zero point fluctuations. a) Predictions for ideal cavity and mechanical occupations at $10 \mathrm{mK}: n_{c}^{\text {th }}=0 ; n_{m}^{\text {th }}=50$. b) Predictions for cavity and mechanical occupations including heating: $n_{c}^{t h}=0.7 ; n_{m}^{t h}=600$.

associated with the net damping, the back-action is reduced by a factor of $2 G_{+}\left(G_{-}-G_{+}\right) / \mathcal{G}^{2}$ for $\hat{X}_{1}$ and increased by a factor of $2 G_{+}\left(G_{-}+G_{+}\right) / \mathcal{G}^{2}$ for $\hat{X}_{2}$. This reduction for $\hat{X}_{1}$ makes it possible to reduce $\left\langle\hat{X}_{1}^{2}\right\rangle$ below $\left\langle\hat{X}_{1}^{2}\right\rangle_{Z P}=1 / 2$.

Fig. 2.3 shows the predicted squeezing for our typical device parameters under ideal conditions and with heating. Note that the optimum drive ratio tends to increase with increasing power.

\subsubsection{General case}

In general, to calculate $\left\langle\hat{X}_{1}^{2}\right\rangle$ when $\delta$ and $\Delta$ are non-zero, we must calculate the integral

$$
\begin{aligned}
\left\langle\hat{X}_{1}^{2}\right\rangle=\frac{1}{2 \pi} \int_{-\infty}^{\infty} d \omega\{ & \kappa\left(\mathbf{D}_{31}[\omega] \mathbf{D}_{31}[-\omega]+\mathbf{D}_{32}[\omega] \mathbf{D}_{32}[-\omega]\right)\left(n_{c}^{t h}+1 / 2\right) \\
& +i \kappa\left(\mathbf{D}_{31}[\omega] \mathbf{D}_{32}[-\omega]-\mathbf{D}_{32}[\omega] \mathbf{D}_{31}[-\omega]\right) / 2 \\
& +\gamma_{m}\left(\mathbf{D}_{33}[\omega] \mathbf{D}_{33}[-\omega]+\mathbf{D}_{34}[\omega] \mathbf{D}_{34}[-\omega]\right)\left(n_{m}^{t h}+1 / 2\right) \\
& \left.+i \gamma_{m}\left(\mathbf{D}_{33}[\omega] \mathbf{D}_{34}[-\omega]-\mathbf{D}_{34}[\omega] \mathbf{D}_{33}[-\omega]\right) / 2\right\}
\end{aligned}
$$

To simplify this expression, it is useful to note that the terms on the second and fourth lines are odd functions of $\omega$, and thus integrate to 0 . Moreover, $\mathbf{D}[-\omega]=\mathbf{D}^{*}[\omega]$, and so we can write $\left\langle\hat{X}_{1}^{2}\right\rangle$ as

$$
\begin{aligned}
\left\langle\hat{X}_{1}^{2}\right\rangle=\frac{1}{2 \pi} \int_{-\infty}^{\infty} d \omega\{ & \kappa\left(\left|\mathbf{D}_{31}[\omega]\right|^{2}+\left|\mathbf{D}_{32}[\omega]\right|^{2}\right)\left(n_{c}^{t h}+1 / 2\right) \\
& \left.+\gamma_{m}\left(\left|\mathbf{D}_{33}[\omega]\right|^{2}+\left|\mathbf{D}_{34}[\omega]\right|^{2}\right)\left(n_{m}^{t h}+1 / 2\right)\right\} .
\end{aligned}
$$




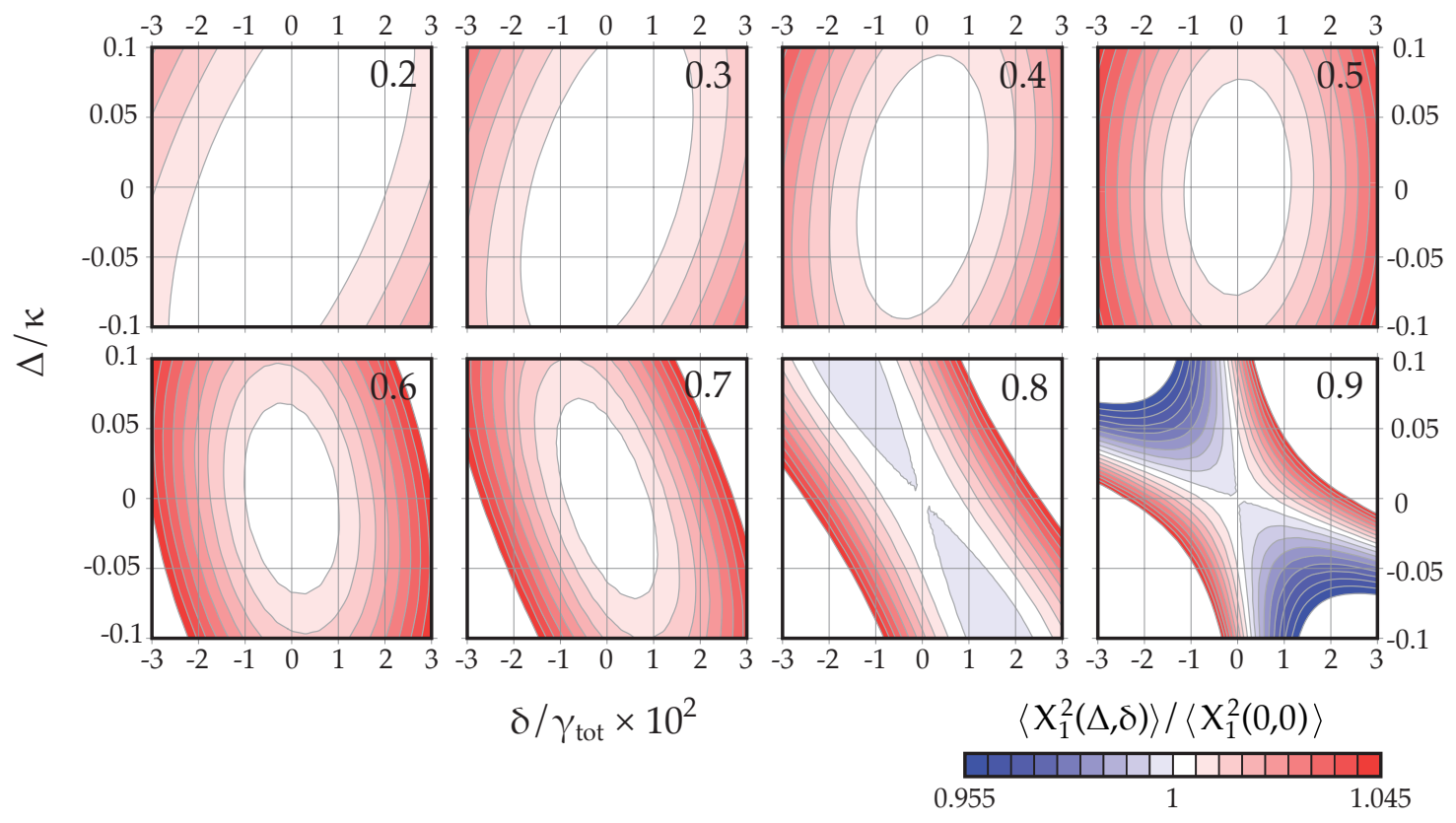

Figure 2.4: Effects of detuning on $\left\langle\hat{X}_{1}^{2}\right\rangle$ for different pump ratios. For all plots, $\gamma_{m}=3 \times 10^{-5} \kappa$, $n_{c}^{t h}=0.7, n_{m}^{t h}=600$, and the total cooperativity is Cctot $=10^{4}$. The pump power ratio $n_{p}^{+} / n_{p}^{-}=$ $\left(G_{+} / G_{-}\right)^{2}$ is shown in the upper-right corner of each plot. $\Delta / \kappa$ is plotted on each y-axis from -0.1 to $0.1 . \delta / \gamma_{t o t}$ is plotted on each $\mathrm{x}$-axis from -0.03 to 0.03 . Note that $\gamma_{t o t}=\gamma_{m}+4 \mathcal{G}^{2} / \kappa$, the total mechanical linewidth, is different for each pump ratio. Values of $\left\langle\hat{X}_{1}^{2}(\Delta, \delta)\right\rangle$, normalized by $\left\langle\hat{X}_{1}^{2}\right\rangle$ evaluated at $\Delta=\delta=0$, are shown as contours, with red indicating an increase in $\left\langle\hat{X}_{1}^{2}\right\rangle$, and blue indicating a reduction. As the ratio increases, the detunings produce more of an effect on $\left\langle\hat{X}_{1}^{2}\right\rangle$. For high ratios, some combinations of detunings can produce a reduction of $\left\langle\hat{X}_{1}^{2}\right\rangle$.

When either $\delta=0$ or $\Delta=0$, this integral can be solved analytically. In the fully-general case, it is easier to solve numerically. Fig. 2.4 shows the effects of imperfect alignment on $\left\langle\hat{X}_{1}^{2}\right\rangle$.

\subsection{Driven response}

One way to measure the parameters of our system is through taking a driven response with a network analyzer. The vector network analyzer (VNA) sends a small probe tone into the left port of our device and measures the relative amplitude and phase of the tone when it exits the right port. The VNA sweeps the frequency of the probe to measure the complex transmission $\left(S_{21}\right)$ as a function of frequency.

We could theoretically calculate $S_{21}$ for our system completely classically, but it is also straightforward to use Eq. 2.27 by setting all the input noise terms equal to 0 apart from $\hat{d}_{i n, L}=\alpha_{i n, L}$. We can then solve for $S_{21}=\alpha_{\text {out }, R} / \alpha_{\text {in }, L}$.

Since $\hat{d}_{\text {out }, R}=\hat{d}_{i n, R}+\sqrt{\kappa_{R}} \hat{d}$, we care only about the component of $\mathbf{B}=\mathbf{A}^{-1}$ that gives the 
$\hat{d}_{i n}[\omega]$ dependence of $\hat{d}[\omega], \mathbf{B}_{11}$ :

$$
\begin{gathered}
S_{21}(\omega)=\alpha_{o u t, R} / \alpha_{i n, L}=-\sqrt{\kappa_{R} \kappa_{L}} \mathbf{B}_{11} \\
\mathbf{B}_{11}=f[\omega]^{-1}\left[G_{-}^{2} \chi_{m}^{-1}[\omega+\delta]-G_{+}^{2} \chi_{m}^{-1}[\omega-\delta]+\chi_{c}^{-1}[\omega-\Delta] \chi_{m}^{-1}[\omega+\delta] \chi_{m}^{-1}[\omega-\delta]\right] \\
f[\omega]=\mathcal{G}^{4}+\chi_{c}^{-1}[\omega+\Delta]\left(G_{-}^{2} \chi_{m}^{-1}[\omega+\delta]-G_{+}^{2} \chi_{m}^{-1}[\omega-\delta]\right) \\
+\chi_{c}^{-1}[\omega-\Delta]\left(G_{-}^{2} \chi_{m}^{-1}[\omega-\delta]-G_{+}^{2} \chi_{m}^{-1}[\omega+\delta]\right) \\
+\chi_{c}^{-1}[\omega+\Delta] \chi_{c}^{-1}[\omega-\Delta] \chi_{m}^{-1}[\omega+\delta] \chi_{m}^{-1}[\omega-\delta] .
\end{gathered}
$$

Eq. 2.43 is a general model of the driven response of the system, and can be easily adapted for the case of a single red-detuned pump $\left(G_{+} \rightarrow 0\right)$, a single blue-detuned pump $\left(G_{-} \rightarrow 0\right)$, or no pumps $\left(G_{+}, G_{-} \rightarrow 0\right)$. In the latter case, it is easy to confirm that $\mathbf{B}_{11}=\chi_{c}[\omega]$ as expected. Fig. 2.5 has example driven responses for various pump ratios and detunings.

In the squeezing experiment, we are usually operating in the regime where $\gamma_{m} \ll \mathcal{G}, \kappa$, and so it is valid to approximate $\gamma_{m}=0$ in $\chi_{m}$. In this case, we obtain

$$
\begin{aligned}
& \mathbf{B}_{11}=f[\omega]^{-1}\left[-i\left(\omega \mathcal{G}^{2}+\delta \mathcal{G}_{\text {tot }}^{2}\right)-\left(\omega^{2}-\delta^{2}\right) \chi_{c}^{-1}[\omega-\Delta]\right] \\
& f[\omega]=\mathcal{G}^{4}-2 i \mathcal{G}^{2} \omega \chi_{c}^{-1}[\omega]-2 \mathcal{G}_{\text {tot }}^{2} \delta \Delta-\left(\omega^{2}-\delta^{2}\right) \chi_{c}^{-1}[\omega+\Delta] \chi_{c}^{-1}[\omega-\Delta] .
\end{aligned}
$$

\subsection{Output spectrum}

We measure the output field with a spectrum analyzer, which is equivalent to measuring the symmetrized current spectral density in the lab frame:

$$
\begin{aligned}
\bar{S}_{I I}[\omega] & =\frac{1}{2} \int d t\langle\{I(t), I(0)\}\rangle e^{i \omega t} \\
& =\frac{1}{2} \int \frac{d \omega^{\prime}}{2 \pi}\left\langle\left\{I[\omega], I\left[\omega^{\prime}\right]\right\}\right\rangle,
\end{aligned}
$$



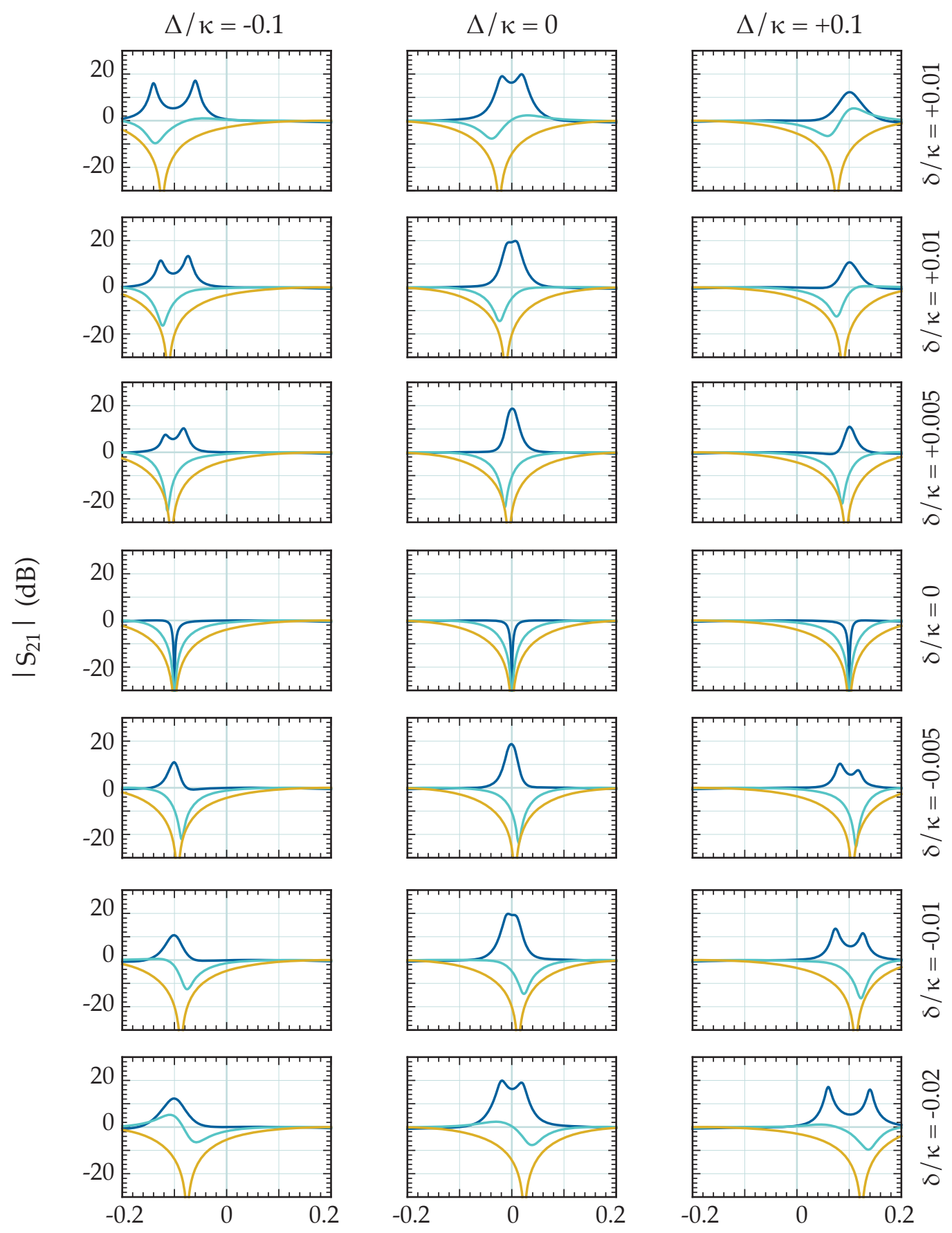

Figure 2.5: $S_{21}$ for two tones at different detunings. For all plots, $\gamma_{m}=3 \times 10^{-5} \kappa$, and $\mathcal{C}_{\text {tot }}=10^{4}$. Blue responses are for $\left(G_{+} / G_{-}\right)^{2}=0.9$, turquoise are for $\left(G_{+} / G_{-}\right)^{2}=0.5$, and yellow are for $\left(G_{+} / G_{-}\right)^{2}=0.1$. Each row has a fixed value of $\delta / \kappa$, as indicated to the right of each row. Each column has a fixed value of $\Delta / \kappa$, as indicated at the top of each column. 
where $I=\hat{d}_{\text {out }, R}+\hat{d}_{\text {out }, R}^{\dagger}$, and $\hat{d}_{\text {out }, R}=\hat{d}_{\text {in }, R}+\sqrt{\kappa_{R}} \hat{d}$. The output operators in the lab frame can be written in terms of the cavity operators in the rotating frame:

$$
\begin{aligned}
& \hat{d}_{\text {out }, R}[\omega]=\hat{d}_{\text {in,R }}[\omega]+\sqrt{\kappa_{R}} \hat{d}\left[\omega-\Omega_{c}\right] \\
& \hat{d}_{\text {out }, R}^{\dagger}[\omega]=\hat{d}_{i n, R}^{\dagger}[\omega]+\sqrt{\kappa_{R}} \hat{d}^{\dagger}\left[\omega+\Omega_{c}\right],
\end{aligned}
$$

where $\Omega_{c}=\omega_{c}+\Delta$ is the frequency of the rotating frame for the cavity operators. In terms of the rotating-frame matrix $\mathbf{B}$, the output field operator in the lab frame is given by:

$$
\begin{aligned}
\hat{d}_{o u t, R}[\omega]=\hat{d}_{i n, R}[\omega] & -\sqrt{\kappa_{R} \kappa}\left(\mathbf{B}_{11}\left[\omega-\Omega_{c}\right] \hat{d}_{i n}\left[\omega-\Omega_{c}\right]+\mathbf{B}_{12}\left[\omega-\Omega_{c}\right] \hat{d}_{i n}^{\dagger}\left[\omega-\Omega_{c}\right]\right) \\
& -\sqrt{\kappa_{R} \gamma_{m}}\left(\mathbf{B}_{13}\left[\omega-\Omega_{c}\right] \hat{c}_{i n}\left[\omega-\Omega_{c}\right]+\mathbf{B}_{14}\left[\omega-\Omega_{c}\right] \hat{c}_{i n}^{\dagger}\left[\omega-\Omega_{c}\right]\right) .
\end{aligned}
$$

When we measure $\bar{S}_{I I}[\omega]$, we can only access positive frequencies. Thus, we only care about the terms peaked at $+\omega_{c}$. This allows us to only consider the contributions from the terms $\left\langle\left\{\hat{d}_{\text {out }, R}[\omega], \hat{d}_{\text {out }, R}^{\dagger}\left[\omega^{\prime}\right]\right\}\right\rangle$.

We can thus calculate the relevant expectation values:

$$
\begin{aligned}
\frac{1}{2 \pi}\left\langle\hat{d}_{\text {out }, R}[\omega] \hat{d}_{\text {out }, R}^{\dagger}\left[\omega^{\prime}\right]\right\rangle= & \delta\left[\omega+\omega^{\prime}\right]\left\{\left(n_{c, R}^{t h}+1\right)-\kappa_{R}\left(\mathbf{B}_{11}\left[\omega-\Omega_{c}\right]+\mathbf{B}_{11}^{*}\left[-\omega^{\prime}-\Omega_{c}\right]\right)\left(n_{c, R}^{t h}+1\right)\right. \\
& +\kappa_{R} \kappa\left(\mathbf{B}_{11}\left[\omega-\Omega_{c}\right] \mathbf{B}_{11}^{*}\left[-\omega^{\prime}-\Omega_{c}\right]\left(n_{c}^{t h}+1\right)+\mathbf{B}_{12}\left[\omega-\Omega_{c}\right] \mathbf{B}_{12}^{*}\left[-\omega^{\prime}-\Omega_{c}\right] n_{c}^{t h}\right) \\
& \left.+\kappa_{R} \gamma_{m}\left(\mathbf{B}_{13}\left[\omega-\Omega_{c}\right] \mathbf{B}_{13}^{*}\left[-\omega^{\prime}-\Omega_{c}\right]\left(n_{m}^{t h}+1\right)+\mathbf{B}_{14}\left[\omega-\Omega_{c}\right] \mathbf{B}_{14}^{*}\left[-\omega^{\prime}-\Omega_{c}\right] n_{m}^{t h}\right)\right\} \\
\frac{1}{2 \pi}\left\langle\hat{d}_{\text {out }, R}^{\dagger}\left[\omega^{\prime}\right] \hat{d}_{\text {out }, R}[\omega]\right\rangle= & \delta\left[\omega+\omega^{\prime}\right]\left\{n_{c, R}^{t h}-\kappa_{R}\left(\mathbf{B}_{11}\left[\omega-\Omega_{c}\right]+\mathbf{B}_{11}^{*}\left[-\omega^{\prime}-\Omega_{c}\right]\right) n_{c, R}^{t h}\right. \\
& +\kappa_{R} \kappa\left(\mathbf{B}_{11}\left[\omega-\Omega_{c}\right] \mathbf{B}_{11}^{*}\left[-\omega^{\prime}-\Omega_{c}\right] n_{c}^{t h}+\mathbf{B}_{12}\left[\omega-\Omega_{c}\right] \mathbf{B}_{12}^{*}\left[-\omega^{\prime}-\Omega_{c}\right]\left(n_{c}^{t h}+1\right)\right) \\
& \left.+\kappa_{R} \gamma_{m}\left(\mathbf{B}_{13}\left[\omega-\Omega_{c}\right] \mathbf{B}_{13}^{*}\left[-\omega^{\prime}-\Omega_{c}\right] n_{m}^{t h}+\mathbf{B}_{14}\left[\omega-\Omega_{c}\right] \mathbf{B}_{14}^{*}\left[-\omega^{\prime}-\Omega_{c}\right]\left(n_{m}^{t h}+1\right)\right)\right\}
\end{aligned}
$$

and the final output spectrum:

$$
\begin{aligned}
\bar{S}_{I I}[\omega]=( & \left.n_{c, R}^{t h}+1 / 2\right)-2 \kappa_{R} \operatorname{Re}\left[\mathbf{B}_{11}\left[\omega-\Omega_{c}\right]\right]\left(n_{c, R}^{t h}+1 / 2\right) \\
& +\kappa_{R} \kappa\left(\left|\mathbf{B}_{11}\left[\omega-\Omega_{c}\right]\right|^{2}+\left|\mathbf{B}_{12}\left[\omega-\Omega_{c}\right]\right|^{2}\right)\left(n_{c}^{t h}+1 / 2\right) \\
& +\kappa_{R} \gamma_{m}\left(\left|\mathbf{B}_{13}\left[\omega-\Omega_{c}\right]\right|^{2}+\left|\mathbf{B}_{14}\left[\omega-\Omega_{c}\right]\right|^{2}\right)\left(n_{m}^{t h}+1 / 2\right) .
\end{aligned}
$$

Fig. 2.6 has example driven responses for various pump detunings to show how imperfect pump alignment affects the measured spectrum. 
$\Delta / \kappa=-0.1$
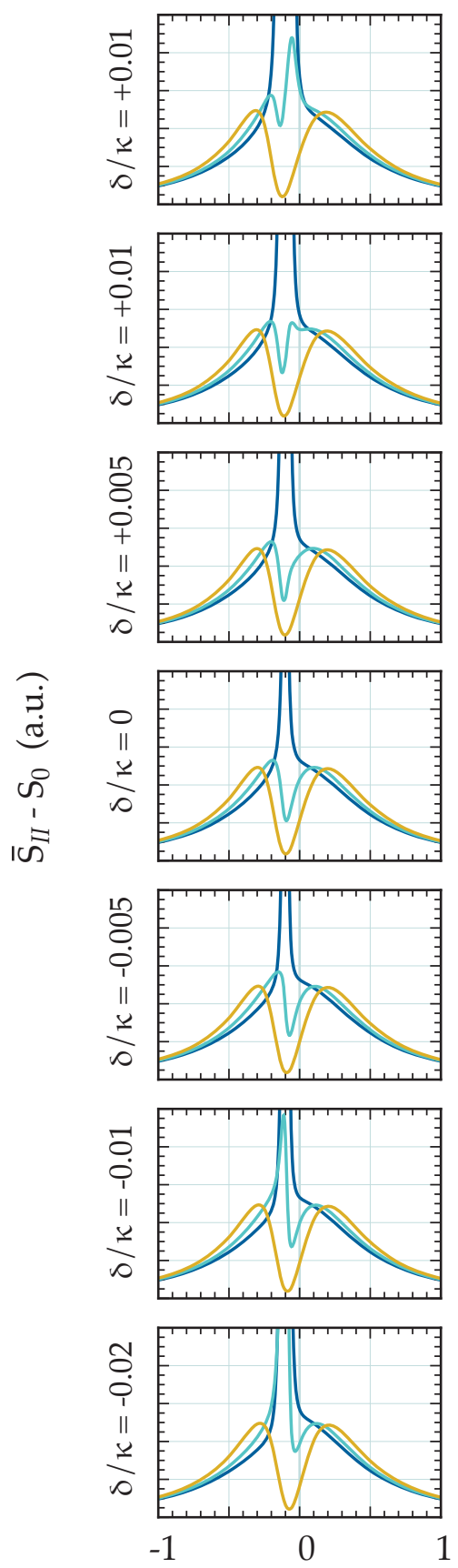

$\Delta / \kappa=0$
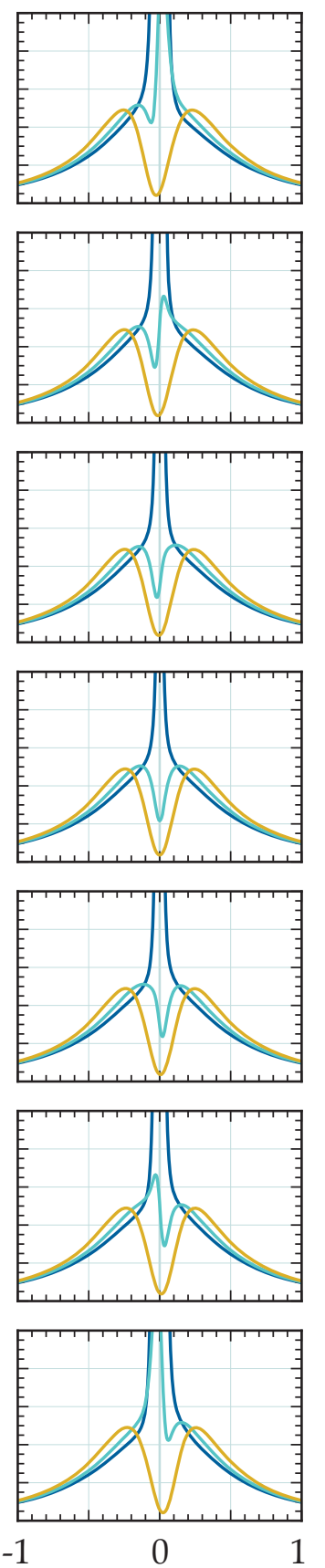

$\left(\omega-\omega_{\mathrm{c}}\right) / \kappa$
$\Delta / \kappa=+0.1$
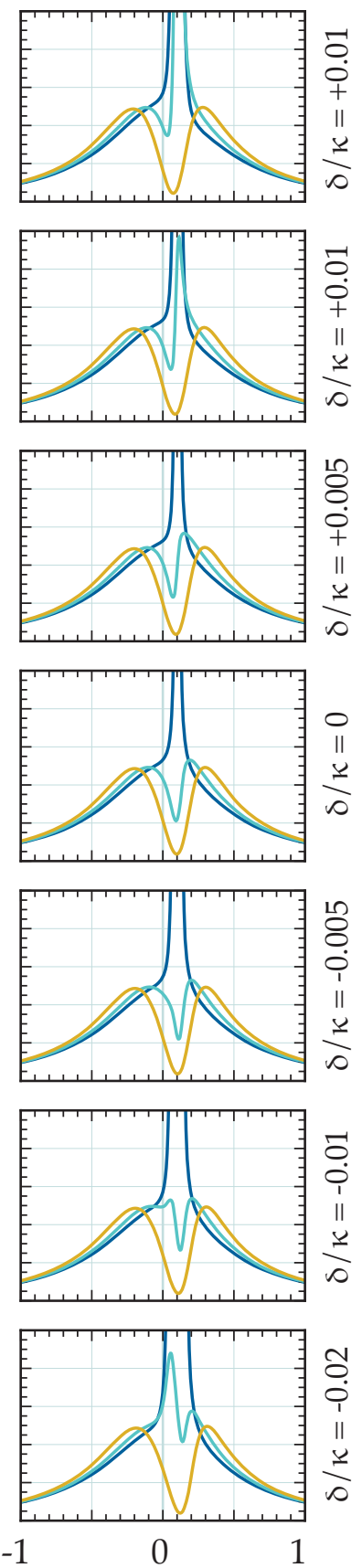

Figure 2.6: $\bar{S}_{I I}-S_{0}$ for two tones at different detunings. For all plots, $\gamma_{m}=3 \times 10^{-5} \kappa$, and $\mathcal{C}_{\text {tot }}=10^{4}$. Blue responses are for $\left(G_{+} / G_{-}\right)^{2}=0.9$, turquoise are for $\left(G_{+} / G_{-}\right)^{2}=0.5$, and yellow are for $\left(G_{+} / G_{-}\right)^{2}=0.1$. Each row has a fixed value of $\delta / \kappa$, as indicated to the left or right of each row. Each column has a fixed value of $\Delta / \kappa$, as indicated at the top of each column. At the highest ratio, the mechanical response always appears as a peak on top of the cavity response. For the intermediate ratio, the mechanical response appears as a dip at $\delta=\Delta=0$, but can become a peak for imperfect detuning. For the lowest ratio, the mechanical response is always a dip. 


\subsubsection{Special case: $\Delta=\delta=0$}

When $\Delta=\delta=0$, the $\mathbf{B}$ matrix simplifies, and we can write the photon annihilation operator as

$$
\hat{d}=-\frac{\chi_{m}^{-1}}{\mathcal{G}^{2}+\chi_{m}^{-1} \chi_{c}^{-1}} \sqrt{\kappa} \hat{d}_{i n}+\frac{i G_{-}}{\mathcal{G}^{2}+\chi_{m}^{-1} \chi_{c}^{-1}} \sqrt{\gamma_{m}} \hat{c}_{i n}+\frac{i G_{+}}{\mathcal{G}^{2}+\chi_{m}^{-1} \chi_{c}^{-1}} \sqrt{\gamma_{m}} \hat{c}_{i n}^{\dagger}
$$

Applying Eq. 2.49 then gives

$$
\begin{aligned}
\bar{S}_{I I}[\omega]=\left(n_{c, R}^{t h}\right. & +1 / 2)-\kappa_{R} \frac{\gamma_{m} \mathcal{G}^{2}+\kappa\left(\left(\gamma_{m} / 2\right)^{2}+\left(\omega-\Omega_{c}\right)^{2}\right)}{\left|g\left[\omega-\Omega_{c}\right]\right|^{2}} n_{c, R}^{t h} \\
& +\kappa_{R} \kappa \frac{\left(\gamma_{m} / 2\right)^{2}+\left(\omega-\Omega_{c}\right)^{2}}{\left|g\left[\omega-\Omega_{c}\right]\right|^{2}} n_{c}^{t h}+\kappa_{R} \gamma_{m} \frac{G_{-}^{2} n_{m}^{t h}+G_{+}^{2}\left(n_{m}^{t h}+1\right)}{\left|g\left[\omega-\Omega_{c}\right]\right|^{2}} \\
g[\omega] & =\mathcal{G}^{2}+\left(\gamma_{m} / 2-i \omega\right)(\kappa / 2-i \omega) .
\end{aligned}
$$

For Device $2, \gamma_{m} \ll \mathcal{G}, \kappa$ and $n_{m}^{t h} \gg 1$ at the pump powers we apply for squeezing. Moreover, $n_{c, R}^{t h}=0$, and thus we can approximate the spectrum, centered at the mean pump frequency $\Omega_{c}$, as

$$
\bar{S}_{I I}\left[\omega+\Omega_{c}\right]=1 / 2+\kappa_{R} \frac{\omega^{2} \kappa n_{c}^{t h}+\left(G_{-}^{2}+G_{+}^{2}\right) \gamma_{m} n_{m}^{t h}}{\left|\mathcal{G}^{2}-i \omega(\kappa / 2-i \omega)\right|^{2}}
$$

If we include the gain of the output line, $G(\omega)$, the signal that we actually measure, in units of $\mathrm{W} / \mathrm{Hz}$, is

$$
S_{\text {out }}\left(\omega+\Omega_{c}\right)=S_{0}+\hbar \omega G(\omega) \kappa_{R} \frac{\omega^{2} \kappa n_{c}^{t h}+\left(G_{-}^{2}+G_{+}^{2}\right) \gamma_{m} n_{m}^{t h}}{\left|\mathcal{G}^{2}-i \omega(\kappa / 2-i \omega)\right|^{2}}
$$

where $S_{0}$ includes both the noise floor of $\bar{S}_{I I}$ and any white noise added by the measurement chain. Since the system gain and $\hbar \omega$ are effectively constant over the linewidth of the cavity, we can write the measured spectrum as

$$
S_{\text {out }}\left(\omega+\Omega_{c}\right)=S_{0}+\hbar \omega_{c} G\left(\omega_{c}\right) \kappa_{R} \frac{\omega^{2} \kappa n_{c}^{t h}+\left(G_{-}^{2}+G_{+}^{2}\right) \gamma_{m} n_{m}^{t h}}{\left|\mathcal{G}^{2}-i \omega(\kappa / 2-i \omega)\right|^{2}}
$$

\subsubsection{Weak driving regime}

For our thermal calibration (Section 3.4.2), we drive the system weakly with two tones of equal amplitude, such that $G_{+}=G_{-}$. In the weak-driving regime, it is useful to define the optical damping rate, $\gamma_{o p}^{ \pm}=4 G_{ \pm}^{2} / \kappa$. The total mechanical linewidth is then $\gamma_{t o t}=\gamma_{m}+\gamma_{o p}^{-}-\gamma_{o p}^{+}=\gamma_{m}+\gamma_{o p}^{\text {eff }}$.

For the thermal calibration, the pumps are positioned so that $\kappa \gg \delta \gg \gamma_{t o t}$. In this case, we can approximate the cavity response, $\chi_{c}[\omega]$, as a constant over the linewidth of the mechanical sideband. We can thus evaluate all cavity susceptibilities at $\omega=\omega_{c}+\Delta \pm \delta$, where the minus (plus) sign is for the sideband of the red-detuned (blue-detuned) pump. As long as $\delta \ll \kappa$, then 
$\chi_{c}^{-1}\left(\omega-\omega_{c}\right)=\kappa / 2-i \Delta$. Moreover, as the mechanical sidebands are assumed to be separated by many mechanical linewidths, we can safely take the product of the red and blue mechanical sideband susceptibilities to be 0 . With these assumptions, we have

$$
\begin{aligned}
& \bar{S}_{I I}\left[\omega+\Omega_{c}-\delta\right]=\left(n_{c, R}^{t h}+1 / 2\right)+4 \frac{\kappa_{R}}{\kappa}\left(n_{c}^{t h \prime}-n_{c, R}^{t h \prime}\right)+\frac{\kappa_{R}}{\kappa} \gamma_{o p}^{-\prime}\left|\tilde{\chi}_{m}^{-}[\omega]\right|^{2} \\
& \times\left\{\gamma_{m} n_{m}^{t h}-2 \gamma_{t o t}\left(n_{c}^{t h \prime}-n_{c, R}^{t h \prime}\right)-\gamma_{t o t} n_{c, R}^{t h}+\gamma_{o p}^{-\prime} n_{c}^{t h \prime}+\gamma_{o p}^{+\prime}\left(n_{c}^{t h \prime}+1\right)\right\} . \quad(2.55) \\
& \bar{S}_{I I}\left[\omega+\Omega_{c}+\delta\right]=\left(n_{c, R}^{t h}+1 / 2\right)+4 \frac{\kappa_{R}}{\kappa}\left(n_{c}^{t h \prime}-n_{c, R}^{t h \prime}\right)+\frac{\kappa_{R}}{\kappa} \gamma_{o p}^{+\prime}\left|\tilde{\chi}_{m}^{+}[\omega]\right|^{2} \\
& \times\left\{\gamma_{m}\left(n_{m}^{t h}+1\right)+2 \gamma_{t o t}\left(n_{c}^{t h \prime}-n_{c, R}^{t h \prime}\right)+\gamma_{t o t} n_{c, R}^{t h}+\gamma_{o p}^{-\prime}\left(n_{c}^{t h \prime}+1\right)+\gamma_{o p}^{+\prime} n_{c}^{t h \prime}\right\}
\end{aligned}
$$

Prime superscripts indicate multiplication by a factor of $\left(1+(2 \Delta / \kappa)^{2}\right)^{-1}$ to take into account the detuning of the average pump frequency. $\gamma_{t o t}$ is now $\gamma_{m}-\gamma_{o p}^{-\prime}+\gamma_{o p}^{+\prime}$, and we have defined

$$
\left|\tilde{\chi}_{m}^{ \pm}[\omega]\right|^{2}=\left\{\left(\omega \pm\left(\gamma_{o p}^{-\prime}+\gamma_{o p}^{+\prime}\right) \Delta / \kappa\right)^{2}+\left(\gamma_{t o t} / 2\right)^{2}\right\}^{-1}
$$

For thermal calibrations, $\gamma_{o p}^{+}=\gamma_{o p}^{-}=\gamma_{o p} \ll \gamma_{m}$ and $n_{c}^{t h}, n_{c, R}^{t h}, 1 \ll n_{m}^{t h}$, so we have

$$
\bar{S}_{I I}\left[\omega+\Omega_{c} \pm \delta\right]=S_{0}+\frac{\kappa_{R}}{\kappa} \frac{\gamma_{o p}^{\prime} \gamma_{m} n_{m}^{t h}}{\gamma_{m}^{2} / 4+\omega^{2}}
$$

which is a Lorentzian with an area proportional to $n_{m}^{t h}$ and a linewidth of $\gamma_{m}$.

\subsection{Back-action evasion}

\subsubsection{Balanced back-action with $\Delta=0$}

In a back-action evading measurement, pumps of equal power are applied at $\omega_{c} \pm \omega_{m}$. In this configuration, the pumps add all their backaction to the $\hat{X}_{2}$ quadrature while performing a measurement

of the $\hat{X}_{1}$ quadrature. From Eqs. 2.38 and 2.39, we find that the quadrature noise terms when $G_{-}=G_{+}=G$ are given by

$$
\begin{aligned}
\left\langle\hat{X}_{1}^{2}\right\rangle & =n_{m}^{t h}+1 / 2 \\
\left\langle\hat{X}_{2}^{2}\right\rangle & =\frac{8 G^{2}}{\gamma_{m}\left(\kappa+\gamma_{m}\right)}\left(n_{c}^{t h}+1 / 2\right)+n_{m}^{t h}+1 / 2 .
\end{aligned}
$$


$\hat{X}_{1}$ is thus unaffected by the measurement, but $\hat{X}_{2}$ contains back-action noise due to both the classical $\left(n_{c}^{t h}\right)$ and quantum $(1 / 2)$ noise in the cavity. The output spectrum for a BAE measurement is obtained by applying $G_{-}=G_{+}=G$ to Eq. 2.51 :

$$
\bar{S}_{I I}[\omega]=\left(n_{c, R}^{t h}+1 / 2\right)+\kappa_{R} \kappa\left|\chi_{c}\right|^{2}\left(n_{c}^{t h}-n_{c, R}^{t h}\right)+\kappa_{R} \gamma_{m}\left|\chi_{c}\right|^{2}\left|\chi_{m}\right|^{2} G^{2}\left(2 n_{m}^{t h}+1\right) .
$$

For the BAE measurements described in Chapter 4 , the linewidth of the mechanics is $\ll \kappa$. When we measure the BAE sideband, we only care about frequencies on the scale of the mechanical linewidth, so we can approximate the cavity susceptibility as constant over the mechanical response: $\chi_{c} \approx(\kappa / 2-i \Delta)^{-1}$. The output spectrum then becomes

$$
\bar{S}_{I I}[\omega]=S_{0}+2 \frac{\kappa_{R}}{\kappa} \gamma_{m} \gamma_{o p}^{\prime}\left|\chi_{m}\right|^{2}\left(n_{m}^{t h}+1 / 2\right)
$$

where $S_{0}$ is a flat spectral background, and the prime superscript again indicates multiplication by a factor of $\left(1+(2 \Delta / \kappa)^{2}\right)^{-1}$. The BAE tones thus produce a sideband with an area proportional to the occupation of $\hat{X}_{1}$. From Eqs. 2.59 and 2.61, we see that two balanced tones detuned by $\pm \omega_{m}$ from the cavity center do indeed perform a measurement of $\hat{X}_{1}$ without adding back-action noise to the quadrature.

\subsubsection{Effects of imbalance}

To measure the phase dependence of a squeezed state in Chapter 4, we introduce weaker BAE probe tones and measure their sideband. These probe tones usually have some detuning $\Delta$ from cavity center so that their sideband does not overlap with the pump sideband. As there is always some uncertainty in how well the probe tones are balanced - that is, in how well their intracavity occupations are matched - we need a model of their sideband spectrum when $G_{-} \neq G_{+}$.

For the purposes of the weak probe measurements, we can treat the strong probes as preparing the state with some initial linewidth $\gamma_{m}$ and some initial occuption $n_{m}^{\text {th }}$, which the probes then measure without perturbing the initial state. In general, a full theory with four driving tones would be necessary to describe this system. This approximation, however, has been able to accurately describe past measurements [51].

For the measurements described later in this dissertation, we can again work in the weak-coupling regime where $\gamma_{t o t} \ll \kappa$, and thus $\chi_{c}=(\kappa / 2-i \Delta)^{-1}$. With this definition for $\chi_{c}$, and with $\delta=0$, our photon annihilation operator becomes

$$
\hat{d}=-\frac{\chi_{m}^{-1}}{\mathcal{G}^{2}+\chi_{m}^{-1} \chi_{c}^{-1}} \sqrt{\kappa} \hat{d}_{i n}+\frac{i G_{-}}{\mathcal{G}^{2}+\chi_{m}^{-1} \chi_{c}^{-1}} \sqrt{\gamma_{m}} \hat{c}_{i n}+\frac{i G_{+}}{\mathcal{G}^{2}+\chi_{m}^{-1} \chi_{c}^{-1}} \sqrt{\gamma_{m}} \hat{c}_{i n}^{\dagger}
$$


Applying Eq. 2.49 then gives

$$
\begin{aligned}
& \bar{S}_{I I}\left[\omega+\omega_{c}+\Delta\right]=\left(n_{c, R}^{t h}+1 / 2\right)-\kappa_{R} \frac{\gamma_{m} \mathcal{G}^{2}+\kappa\left(\left(\gamma_{m} / 2\right)^{2}+\omega^{2}\right)}{|\tilde{g}[\omega]|^{2}} n_{c, R}^{t h} \\
& +\kappa_{R} \kappa \frac{\left(\gamma_{m} / 2\right)^{2}+\omega^{2}}{|\tilde{g}[\omega]|^{2}} n_{c}^{t h}+\kappa_{R} \gamma_{m} \frac{G_{-}^{2} n_{m}^{t h}+G_{+}^{2}\left(n_{m}^{t h}+1\right)}{|\tilde{g}[\omega]|^{2}} ; \\
& \tilde{g}[\omega]=\mathcal{G}^{2}+\left(\gamma_{m} / 2-i \omega\right)(\kappa / 2-i \Delta) .
\end{aligned}
$$

We can write $|\tilde{g}[\omega]|^{2}$ as

$$
|\tilde{g}[\omega]|^{2}=\left((\kappa / 2)^{2}+\Delta^{2}\right)\left(\left(\omega-\gamma_{o p}^{e f f} \frac{\Delta}{\kappa}\right)^{2}+\left(\gamma_{o p}^{e f f^{\prime}} / 2+\gamma_{m} / 2\right)^{2}\right),
$$

where $\gamma_{o p}^{e f f}=4 \mathcal{G}^{2} / \kappa=\gamma_{o p}^{-}-\gamma_{o p}^{+}$. For our weak, nearly-balanced probes, the mechanical frequency shift is much smaller than the linewidth of the mechanics that has been broadened by the squeezing pumps. We can thus approximate $\gamma_{o p}^{e f f^{\prime}} \frac{\Delta}{\kappa}=0$. Identifying $\gamma_{t o t}=\gamma_{m}+\gamma_{o p}^{e f f^{\prime}}$, we have

$$
\begin{aligned}
\bar{S}_{I I}\left[\omega+\omega_{c}+\Delta\right]= & \left(n_{c, R}^{t h}+1 / 2\right)-\frac{\kappa_{R}}{\kappa} \frac{\gamma_{m} \gamma_{o p}^{e f f \prime}}{\left(\gamma_{t o t} / 2\right)^{2}+\omega^{2}} n_{c, R}^{t h} \\
& \quad+\frac{4 \kappa_{R}}{\kappa}\left[1-\frac{\left(\gamma_{t o t} / 2\right)^{2}-\left(\gamma_{m} / 2\right)^{2}}{\left(\gamma_{t o t} / 2\right)^{2}+\omega^{2}}\right]\left(n_{c}^{t h \prime}-n_{c, R}^{t h \prime}\right)+\frac{\kappa_{R}}{\kappa} \frac{\gamma_{o p}^{-\prime} \gamma_{m} n_{m}^{t h}+\gamma_{o p}^{+\prime} \gamma_{m}\left(n_{m}^{t h}+1\right)}{\left(\gamma_{t o t} / 2\right)^{2}+\omega^{2}} \\
= & \left(n_{c, R}^{t h}+1 / 2\right)+\frac{4 \kappa_{R}}{\kappa}\left(n_{c}^{t h \prime}-n_{c, R}^{t h \prime}\right) \\
+ & \frac{\kappa_{R}}{\kappa} \frac{\gamma_{m}}{\left(\gamma_{t o t} / 2\right)^{2}+\omega^{2}}\left\{\gamma_{o p}^{-\prime} n_{m}^{t h}+\gamma_{o p}^{+\prime}\left(n_{m}^{t h}+1\right)-\gamma_{o p}^{e f f \prime} n_{c, R}^{t h}-2 \gamma_{o p}^{e f f \prime}\left(n_{c}^{t h \prime}-n_{c, R}^{t h \prime}\right)\right\},
\end{aligned}
$$

where we've used the fact that $\gamma_{o p}^{e f f} \ll \gamma_{m}$ for our measurements. When the probes are balanced, $\gamma_{o p}^{-\prime}=\gamma_{o p}^{+\prime}$, and $\gamma_{o p}^{e f f \prime}=0$, so we retrieve Eq. 2.61. If, instead, $n_{p}^{+}=n_{p}^{-}(1-\epsilon)$, the output spectrum becomes

$$
\bar{S}_{I I}\left[\omega+\omega_{c}+\Delta\right]=S_{0}+2 \frac{\kappa_{R}}{\kappa} \frac{\gamma_{m} \gamma_{o p}^{-\prime}}{\left(\gamma_{t o t} / 2\right)^{2}+\omega^{2}}\left\{n_{m}^{t h}+1 / 2-\frac{\epsilon}{2}\left(n_{m}^{t h}+1+n_{c, R}^{t h}+2\left(n_{c}^{t h \prime}-n_{c, R}^{t h \prime}\right)\right)\right\} .
$$

We see that, when there is more red power than blue power, the sideband area is less than we'd expect for the given mechanical fluctuations, due both to less blue gain than expected, and to squashing from the cavity fluctuations. We would then infer a smaller quadrature variance than the actual variance. When there is more blue power than red power, we'd infer a larger quadrature variance than the actual value. It is thus important to carefully balance the probe tones to measure the correct noise power. 


\subsection{Single, red-detuned tone}

For the purpose of calibration and characterization, we often want to pump the system with a single, red-detuned tone. In this case, $G_{+}$is 0 , and we need only define one detuning. We can thus let $\delta=0$, so that now $\omega_{-}=\omega_{c}+\Delta-\omega_{m}$. Applying these new definitions reduces the number of coupled Langevin equations so that we now have:

$$
\left(\begin{array}{c}
\hat{d}[\omega] \\
\hat{c}[\omega]
\end{array}\right)=-g_{-}[\omega]^{-1}\left(\begin{array}{cc}
\frac{\gamma_{m}}{2}-i \omega & -i G_{-} \\
-i G_{-} & \frac{\kappa}{2}-i(\omega+\Delta)
\end{array}\right)\left(\begin{array}{c}
\sqrt{\kappa} \hat{d}_{i n}[\omega] \\
\sqrt{\gamma_{m}} \hat{c}_{i n}[\omega]
\end{array}\right)
$$

where

$$
g_{-}[\omega]=G_{-}^{2}+(\kappa / 2-i(\omega+\Delta))\left(\gamma_{m} / 2-i \omega\right)
$$

\subsubsection{Cooling}

From Eq. 2.67, the phonon annihilation operator when $\Delta=0$ is

$$
\hat{c}[\omega]=\sqrt{\kappa} \frac{i G_{-}}{g_{-}[\omega]} \hat{d}_{i n}[\omega]-\sqrt{\gamma_{m}} \frac{\kappa / 2-i \omega}{g_{-}[\omega]} \hat{c}_{i n}[\omega]
$$

The mechanical occupation is then given by

$$
\begin{aligned}
\bar{n}_{m} & =\frac{1}{(2 \pi)^{2}} \iint_{-\infty}^{\infty} d \omega d \omega^{\prime}\left\langle\hat{c}^{\dagger}[\omega] \hat{c}\left[\omega^{\prime}\right]\right\rangle \\
& =\frac{1}{2 \pi} \int_{-\infty}^{\infty} d \omega\left\{\kappa \frac{G_{-}^{2}}{\left|G_{-}^{2}+\chi_{m}^{-1}[\omega] \chi_{c}^{-1}[\omega]\right|^{2}} n_{c}^{t h}+\gamma_{m} \frac{(\kappa / 2)^{2}+\omega^{2}}{\left|G_{-}^{2}+\chi_{m}^{-1}[\omega] \chi_{c}^{-1}[\omega]\right|^{2}} n_{m}^{t h}\right\}
\end{aligned}
$$

From the similarities to Eq. 2.37, we find

$$
\begin{aligned}
\bar{n}_{m} & =\frac{4 \kappa G_{-}^{2}}{\left(4 G_{-}^{2}+\gamma_{m} \kappa\right)\left(\kappa+\gamma_{m}\right)} n_{c}^{t h}+\frac{\gamma_{m}\left(4 G_{-}^{2}+\kappa\left(\kappa+\gamma_{m}\right)\right)}{\left(4 G_{-}^{2}+\gamma_{m} \kappa\right)\left(\kappa+\gamma_{m}\right)} n_{m}^{t h} \\
& =\frac{\kappa \gamma_{o p}^{-}}{\gamma_{t o t}\left(\kappa+\gamma_{m}\right)} n_{c}^{t h}+\frac{\gamma_{m}\left(\gamma_{t o t}+\kappa\right)}{\gamma_{t o t}\left(\kappa+\gamma_{m}\right)} n_{m}^{t h}
\end{aligned}
$$

When $\gamma_{m}<\gamma_{t o t} \ll \kappa$, this simplifies to

$$
\bar{n}_{m}=\frac{\gamma_{o p}^{-}}{\gamma_{t o t}} n_{c}^{t h}+\frac{\gamma_{m}}{\gamma_{t o t}} n_{m}^{t h}
$$

So, when the pump strength is weak, $\gamma_{t o t} \sim \gamma_{m}$, and the mechanical occupation is strongly coupled to the mechanical thermal bath. As the pump strength increases and the mechanical damping, $\gamma_{o p}^{-}$, dominates over the coupling to the mechanical bath, $\gamma_{m}$, the mechanical occupation approaches the 


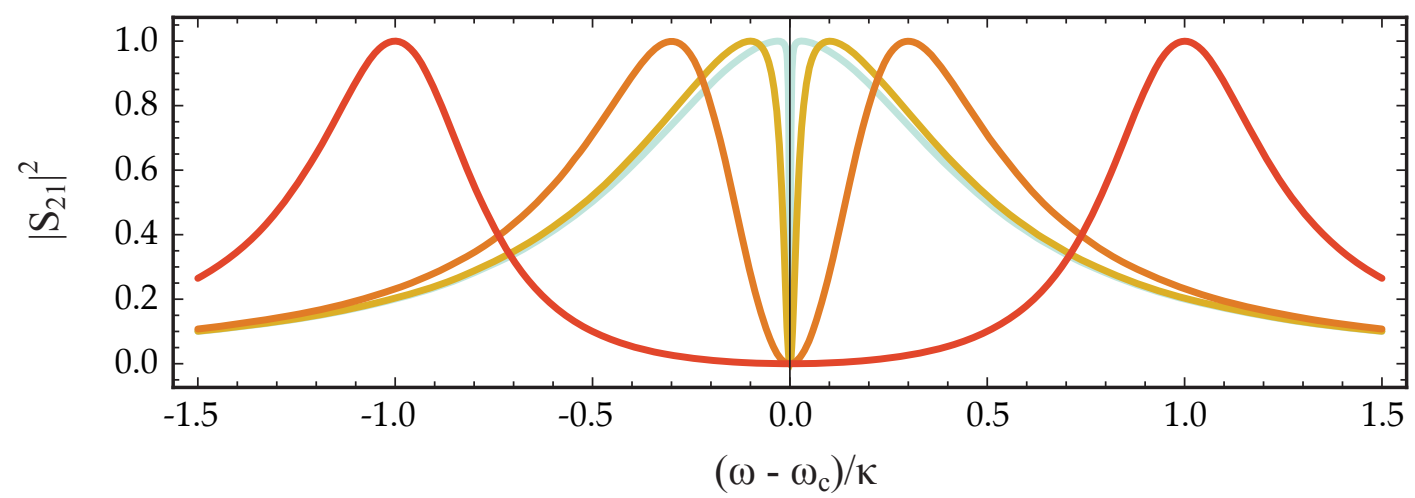

Figure 2.7: Normalized driven responses for a single, red-detuned tone with $\Delta=0$. In this model, $\gamma_{m}=10^{-5} \kappa$, and $G_{-}$is, from turquoise to red, $0.03 \kappa, 0.1 \kappa, 0.3 \kappa$, and $1 \kappa$. In the $1 \kappa$ trace, mode-splitting is evident.

occupation of the cavity bath.

If we include the bad-cavity effects that we neglected when making the rotating wave approximation, the occupation becomes $[15,45]$ :

$$
\bar{n}_{m}=\frac{\gamma_{o p}^{-}}{\gamma_{t o t}}\left(n_{c}^{t h}+2\left(\frac{\kappa}{4 \omega_{m}}\right)^{2} n_{c}^{t h}+\left(\frac{\kappa}{4 \omega_{m}}\right)^{2}\right)+\frac{\gamma_{m}}{\gamma_{t o t}} n_{m}^{t h}
$$

\subsubsection{Driven response}

As in the two-tone case, the first term of the Langevin equation matrix gives us the driven response transmission:

$$
S_{21}[\omega]=-\sqrt{\kappa_{L} \kappa_{R}} \frac{\frac{\gamma_{m}}{2}-i \omega}{G_{-}^{2}+(\kappa / 2-i(\omega+\Delta))\left(\gamma_{m} / 2-i \omega\right)} .
$$

Fig. 2.7 shows the typical electrically-induced transparency (or opacity in our transmission devices) as the pump power increases.

In the weak-driving regime, the mechanical linewidth is much smaller than the cavity linewidth. In taking a driven response of the mechanics, we only care about frequencies near the mechanical sideband frequency. When the sideband is close to the center of the cavity, $\omega+\Delta$ is effectively 0 when compared to $\kappa$. We thus have

$$
\begin{aligned}
S_{21}[\omega] & =-2 \frac{\sqrt{\kappa_{L} \kappa_{R}}}{\kappa} \frac{\frac{\gamma_{m}}{2}-i \omega}{\left(\gamma_{m}+4 G_{-}^{2} / \kappa\right) / 2-i \omega} \\
& =-2 \frac{\sqrt{\kappa_{L} \kappa_{R}}}{\kappa}\left[1-\frac{\gamma_{o p} / 2}{\gamma_{t o t} / 2-i \omega}\right] .
\end{aligned}
$$

This is a Lorentzian response with linewidth $\gamma_{t o t}$. Driven responses of the mechanics are used to 


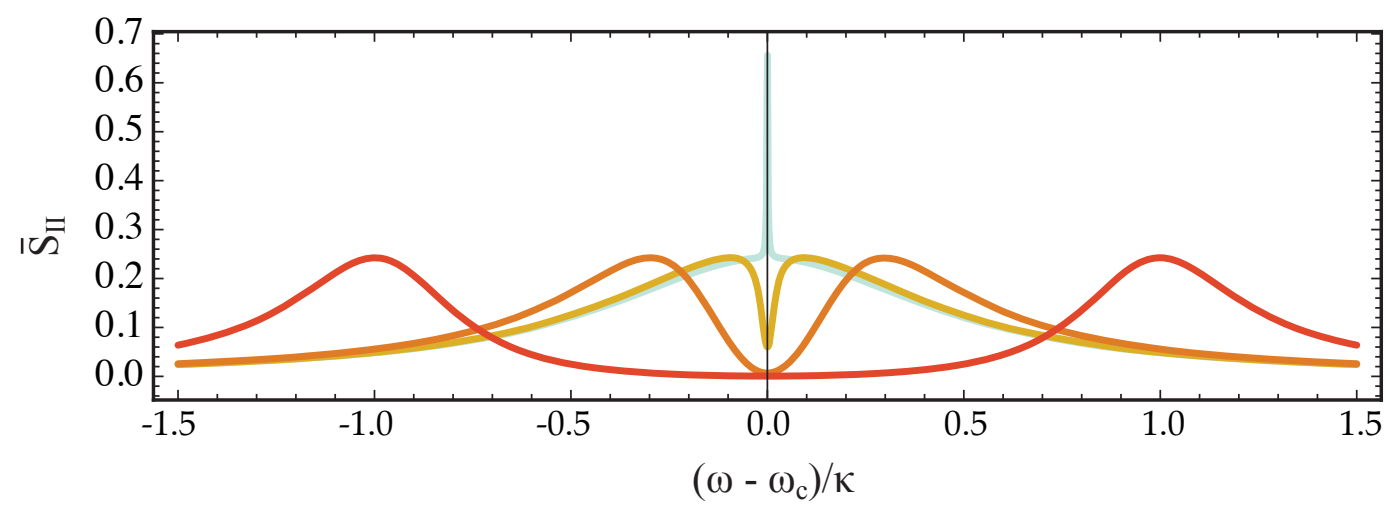

Figure 2.8: Background-subtracted spectral responses for a single, red-detuned tone with $\Delta=0$. As in Fig. 2.7, $\gamma_{m}=10^{-5} \kappa$, and $G_{-}$is, from turquoise to red, $0.03 \kappa, 0.1 \kappa, 0.3 \kappa$, and $1 \kappa$. Additionally, $n_{m}^{t h}=100$ and $n_{c}^{t h}=0.1$. As the mechanical mode is cooled, the squashing from the thermal cavity occupation is more evident, and eventually the mechanical sideband appears as a dip rather than as a peak. At the highest powers, there is mode-splitting due to hybridization between the cavity and mechanical modes.

calibrate $G_{-}$to the measured output power of the pump (Section 3.4.3).

\subsubsection{Spectral response}

Following the same procedure as in Section 2.6, the output spectrum in the single-tone case is given by

$$
\begin{aligned}
\bar{S}_{I I}[\omega]= & \left(n_{c, R}^{t h}+1 / 2\right)-2 \kappa_{R} \operatorname{Re}\left[\mathbf{B}_{11}\left[\omega-\Omega_{c}\right]\right]\left(n_{c, R}^{t h}+1 / 2\right) \\
& +\kappa_{R} \kappa\left|\mathbf{B}_{11}\left[\omega-\Omega_{c}\right]\right|^{2}\left(n_{c}^{t h}+1 / 2\right)+\kappa_{R} \gamma_{m}\left|\mathbf{B}_{12}\left[\omega-\Omega_{c}\right]\right|^{2}\left(n_{m}^{t h}+1 / 2\right),
\end{aligned}
$$

where $\mathbf{B}$ is now the matrix in Eq. 2.67. The output spectrum is then

$$
\bar{S}_{I I}[\omega]=\left(n_{c, R}^{t h}+1 / 2\right)+\kappa_{R} \frac{\kappa\left(\gamma_{m}^{2} / 4+\left(\omega-\omega_{c}-\Delta\right)^{2}\right)\left(n_{c}^{t h}-n_{c, R}^{t h}\right)+\gamma_{m} G_{-}^{2}\left(n_{m}^{t h}-n_{c, R}^{t h}\right)}{\left|G_{-}^{2}+\left(\kappa / 2-i\left(\omega-\omega_{c}\right)\right)\left(\gamma_{m} / 2-i\left(\omega-\omega_{c}-\Delta\right)\right)\right|^{2}} .
$$

Example spectra are shown in Fig. 2.8.

In the weak-coupling limit, and with $\Delta=0$, if we only care about the spectrum near the mechanical response, the output spectrum becomes

$$
\bar{S}_{I I}\left[\omega+\omega_{c}\right]=\left(n_{c, R}^{t h}+1 / 2\right)+\frac{4 \kappa_{R}}{\kappa}\left(n_{c}^{t h}-n_{c, R}^{t h}\right)+\frac{\kappa_{R}}{\kappa} \frac{\gamma_{o p}^{-} \gamma_{t o t}}{\gamma_{t o t}^{2} / 4+\omega^{2}}\left[\bar{n}_{m}-\left(2 n_{c}^{t h}-n_{c, R}^{t h}\right)\right]
$$

where $\bar{n}_{m}=\frac{\gamma_{m}}{\gamma_{t o t}} n_{m}^{t h}+\frac{\gamma_{o p}^{-}}{\gamma_{t o t}} n_{c}^{t h}$ as defined in Eq. 2.72. The sideband area is not simply proportional to $\bar{n}_{m}$, but to $\bar{n}_{m}-\left(2 n_{c}^{t h}-n_{c, R}^{t h}\right)$. The decrease in the sideband area is due to destructive interference between the cavity noise that interacts with the mechanics and the noise that does not interact 
with the mechanics. The effect is commonly referred to as "noise-squashing" [41, 45]. Similarly, "anti-squashing" can occur for the sideband of a blue-detuned pump.

Note that, in the absence of any mechanics, the full spectrum in Eq. 2.77 becomes

$$
\bar{S}_{I I}[\omega]=\left(n_{c, R}^{t h}+1 / 2\right)+\kappa_{R} \kappa \frac{n_{c}^{t h}-n_{c, R}^{t h}}{(\kappa / 2)^{2}+\left(\omega-\omega_{c}\right)^{2}},
$$

which is a Lorentzian with linewidth $\kappa$ and an area proportional to $n_{c}^{t h}-n_{c, R}^{t h}$. If the only cavity bath with non-zero occupation is the right port, then $n_{c}^{t h}=\frac{\kappa_{R}}{\kappa} n_{c, R}^{t h}$, and we have

$$
S_{I I}[\omega]=\left(n_{c, R}^{t h}+1 / 2\right)-\kappa_{R} \frac{\kappa-\kappa_{R}}{(\kappa / 2)^{2}+\left(\omega-\omega_{c}\right)^{2}} n_{c, R}^{t h}
$$

which looks like a Lorentzian dip in the noise floor with linewidth $\kappa$. Observation of such a dip is thus a sign of a hot output port. 


\section{Chapter 3}

\section{Experimental set-up and calibrations}

\subsection{Device}

\subsubsection{Device fabrication}

Our device consists of a lumped-element LC circuit, in which the suspended top gate of the parallelplate capacitor acts as a mechanical resonator due to drum-head resonances in the membrane. $50-\Omega$ coplanar-waveguide transmission lines are coupled to the LC resonator via coupling capacitors at the input and output. We make the output coupling capacitor larger than the input capacitor to improve read-out. Optical and SEM images of a sample device are shown in Fig. 3.1.

The devices are fabricated from aluminum on top of a high-resistivity silicon substrate. The substrate is first cleaned and its native oxide is removed with buffered HF. A $100 \mathrm{~nm}$ layer of aluminum is sputtered on the chip to form the bottom layer of the device. The bottom layer is patterned via photolithography and a wet etch. This layer includes the bottom plate of the capacitor and half of the ground plane. A layer of S1813 photoresist is spun onto the device, patterned via photolithography, thinned with a flood exposure of the whole chip, and developed. This layer protects the aluminum bottom layer and serves as a sacrificial layer for the capacitor gap and inductor cross-overs. A top layer of $100 \mathrm{~nm}$ aluminum is sputtered and patterned in the same manner as the bottom layer. The top layer includes the inductor, the top gate of the capacitor, and the other half of the ground plane. Finally, the sacrificial layer is removed with Remover-PG and the device is dried in a critical-point dryer, leaving the top gate of the capacitor suspended.

Chan U Lei, a graduate student in the Schwab group, developed the fabrication procedure summarized here and made the devices. For further details, see his PhD thesis. 

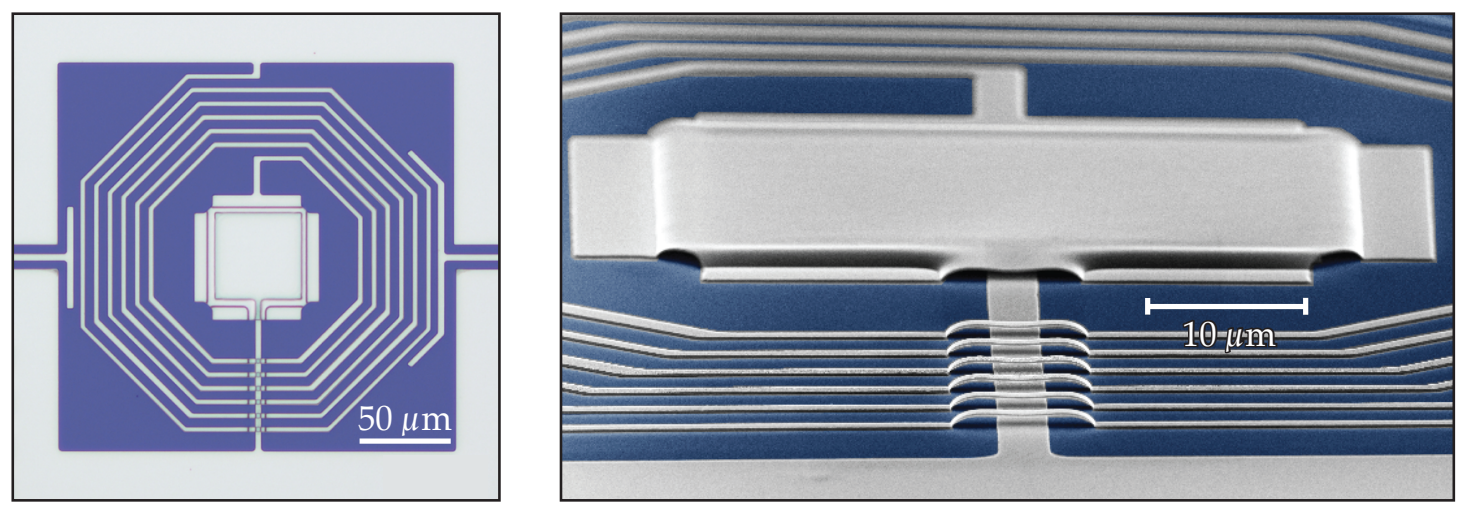

Figure 3.1: Images of a typical device. Left: True-color optical micrograph with aluminum in gray and silicon in blue. The parallel-plate capacitor is the square in the center of the image, while the spiral inductor connects the top plate to ground. The input (left) coupling capacitor can be seen to be smaller than the output (right) coupling capacitor. Right: False-color SEM image of the device. The suspension of the top capacitor plate is evident. The gap height is a few hundred nanometers at room temperature, but contracts to $\sim 100 \mathrm{~nm}$ at low temperatures. At front, the spiral inductor bridges over the bottom plate's connection to ground. These bridges provide some parasitic capacitance.

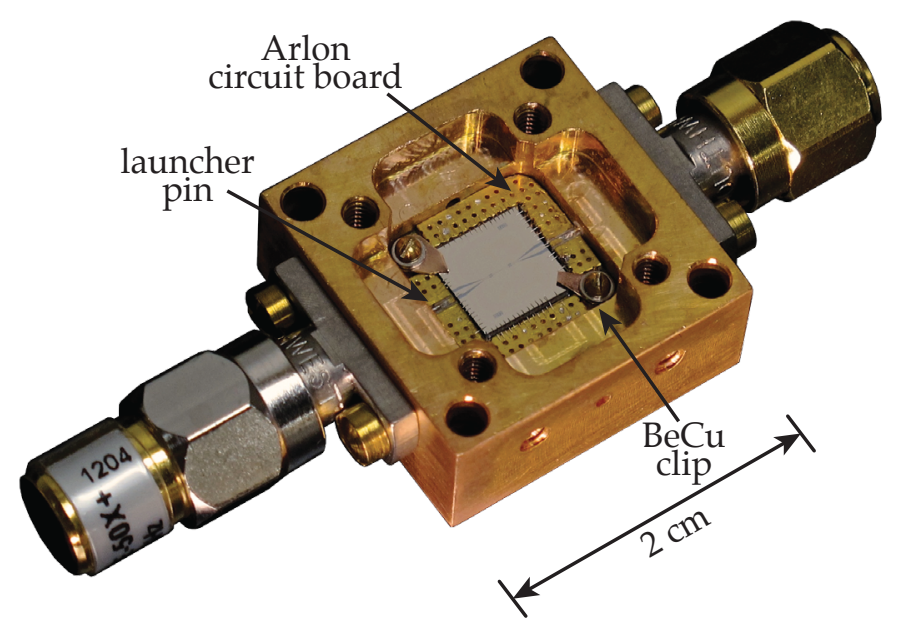

Figure 3.2: Photograph of sample package, showing BeCu clips, printed circuit boards around the chip, and SMA launchers. The chip shown mounted here is a $6 \mathrm{~mm} \times 6 \mathrm{~mm}$ parametric amplifier device, but the same sample package is used for the electromechanical devices. Typical chip sizes are either $6 \times 6 \mathrm{~mm}$ or $3 \times 6 \mathrm{~mm}$. 


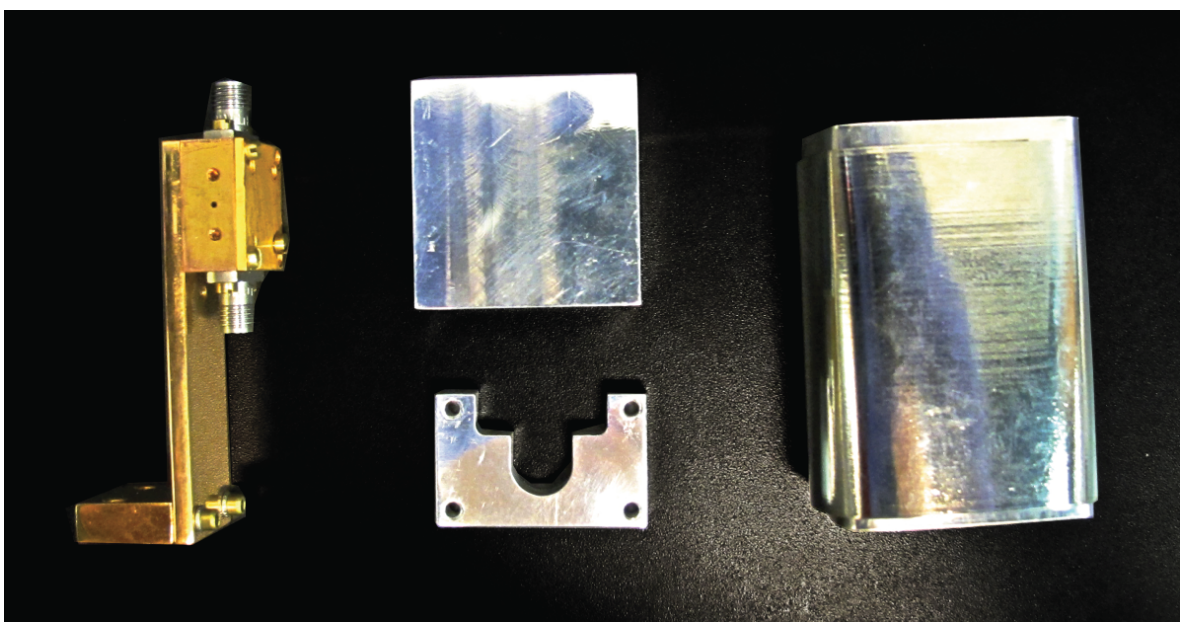

Figure 3.3: Additional aluminum shield (center) fits over both the sample package and cold finger (left), and includes a hole in the top for accessing the output SMA connector. The aluminum cap is then bolted to the bottom, and the whole finger is covered with the mu-metal and Metglas shield (right).

\subsubsection{Sample package and shielding}

The sample is mounted in a gold-plated copper sample package using beryllium copper clips (see Fig. 3.2). For some devices, we also use a small amount of either PMMA photoresist or GE varnish for additional thermalization, but we have not seen a noticeable improvement in performance in these cases. The sample package contains pieces of Arlon circuit board (AR1000) that have been indium-soldered into the box. The chip is wire-bonded to the circuit board using aluminum wire. Southwest Microwave launchers (214-5 series) convert the transmission lines from grounded coplanar waveguide to coaxial SMA connectors.

The entire sample package is mounted on a cold finger and surrounded by a mu-metal shield. For additional magnetic shielding, we have tried using superconducting aluminum boxes around the sample packages, and additional Metglas around the mu-metal shield, but did not find a noticeable difference in performance with the extra shielding. The additional shielding is shown in Fig. 3.3. Typical shielding on devices mounted in the dilution refrigerator is shown in Fig. 3.4.

\subsection{Dilution refrigerator}

All measurements are performed on the same Oxford Instruments Kelvinox 400 dilution refrigerator. With all stages and cables connected, our fridge has a base temperature of $7 \mathrm{mK}$, as confirmed with mechanical thermal measurements described in Section 3.4.2 and [51]. The refrigerator is mounted to a floating optical table for vibration isolation, and is located within a shielded room to minimize electrical noise. 


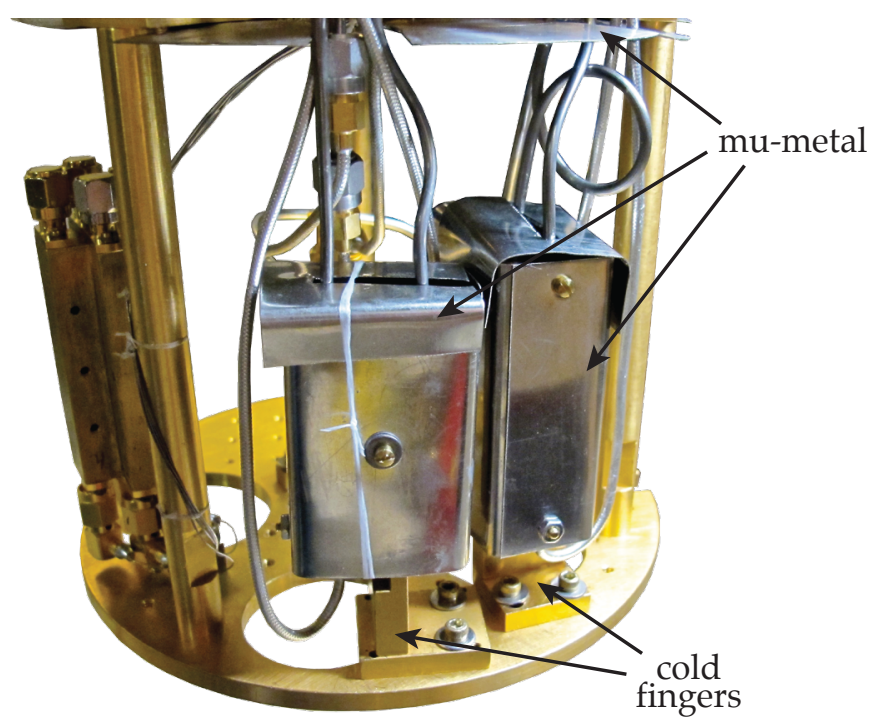

Figure 3.4: Samples mounted in the fridge. The stage shown here is connected to the mixing chamber via gold-plated copper stand-offs. Each of the two cold fingers has two samples packages mounted, and the sample packages are surrounded by mu-metal shielding. There is also a mu-metal plate above this stage, and a cryoperm can around both plates connected to the mixing chamber.

For the purposes of mounting components, the fridge has a stage at $4.2 \mathrm{~K}$ thermally connected to the liquid helium bath (hereafter referred to as the $4 \mathrm{~K}$ stage or $4 \mathrm{~K}$ plate), a stage at $\sim 1.7 \mathrm{~K}$ connected to the $1 \mathrm{~K}$ pot, a stage at $\sim 100 \mathrm{mK}$ connected to the dilution unit heat exchangers, and a stage connected to the mixing chamber which is capable of reaching the fridge's base temperature. The mixing stage temperature is measured with a calibrated ruthenium oxide resistance thermometer and a Picowatt AVS-47B resistance bridge. We can control the temperature of this stage between $20 \mathrm{mK}$ and $1 \mathrm{~K}$ using a Picowatt TS-530A temperature controller.

\subsection{Microwave circuitry}

A simplified circuit diagram for our microwave measurements is shown in Fig. 3.5. Up to three sources are combined at room temperature, filtered, and sent into the fridge. The pumps are attenuated to decrease the room-temperature thermal noise, and then sent into the input port of the device. At the output of the device, the transmitted pump power and noise spectrum from the device are amplified in two stages and then measured. Each part of the this process is discussed further in the following sections. 


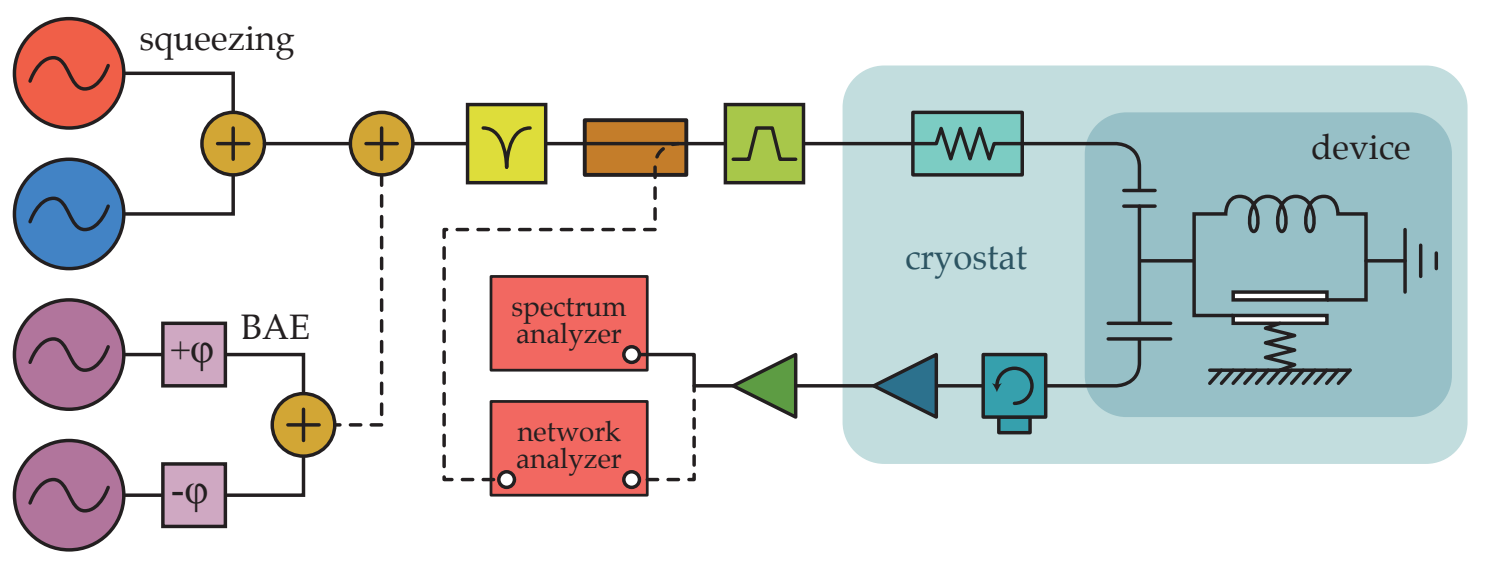

Figure 3.5: Microwave circuit diagram showing both the input and output lines.

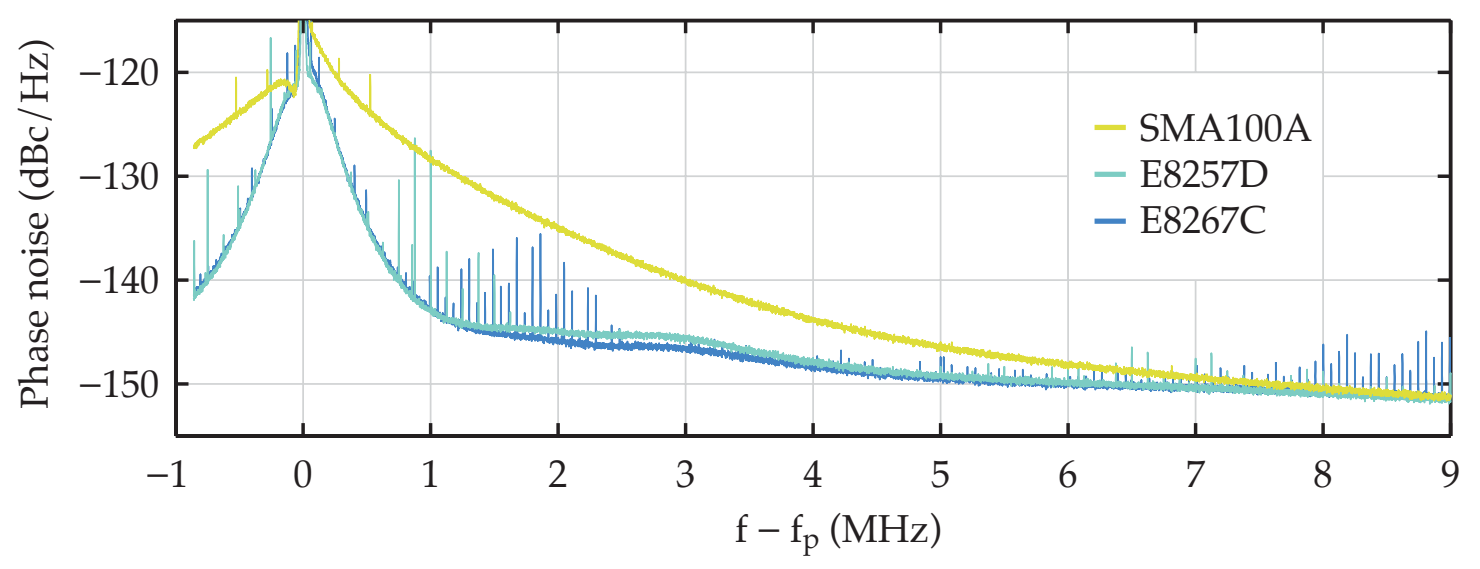

Figure 3.6: Phase noise measurements of the microwave sources used in this experiment. $f_{p}$ is the carrier frequency. We are mainly concerned about the phase noise between 3 and $5 \mathrm{MHz}$ away from the carrier, as the mechanical frequencies of our devices lie within this range.

\subsubsection{Input line: sources, filtering, and attenuation}

The pumps and probes necessary for the squeezing experiment and associated calibrations are all generated by off-the-shelf microwave signal generators. For the experiments described here, we used an Agilent E8267C vector source, an Agilent E8257D signal generator, and a Rohde \& Schwarz SMA100A signal generator. All sources have phase noise at the level of -150 to $-140 \mathrm{dBc} / \mathrm{Hz}$ at 4 $\mathrm{MHz}$ away from the carrier frequency, as shown in Fig. 3.6. In addition, room temperature thermal noise contributes $-174 \mathrm{dBm} / \mathrm{Hz}$. When we are using the sources as low-power probes, this white thermal noise dominates the pump phase noise, and thus no filtering is necessary. If we use the sources at higher powers, however, this phase noise behaves as a hot bath at the cavity frequency and can significantly impact our measurements.

To suppress the pump phase noise below the level of the room-temperature thermal noise, we 


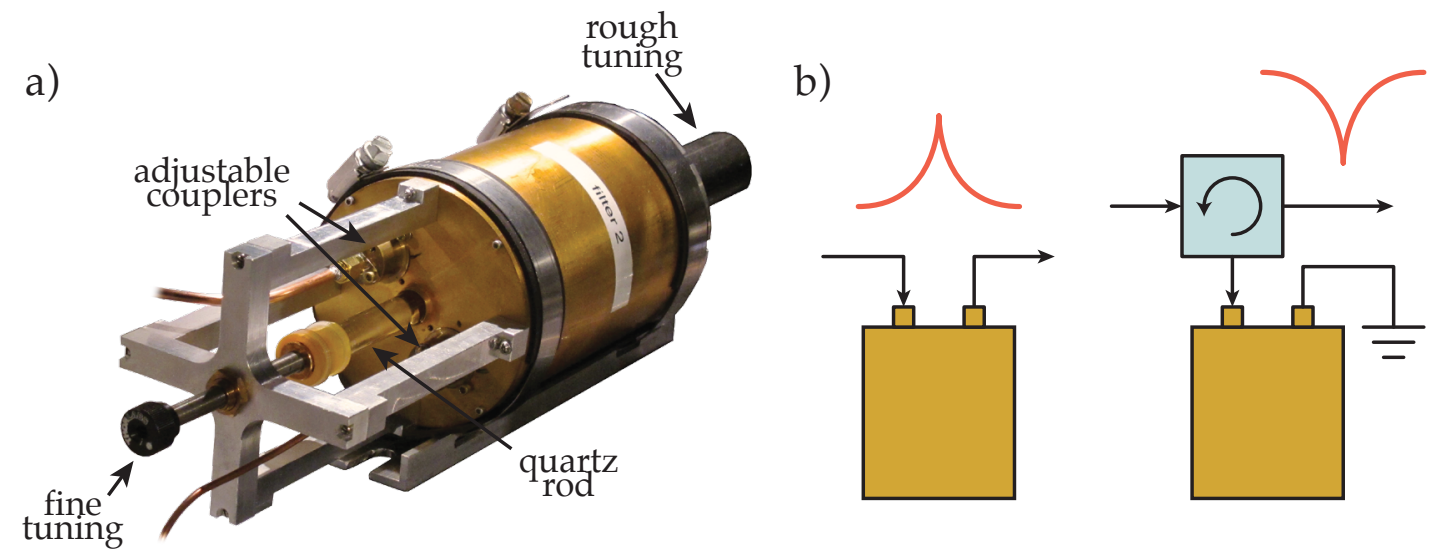

c)

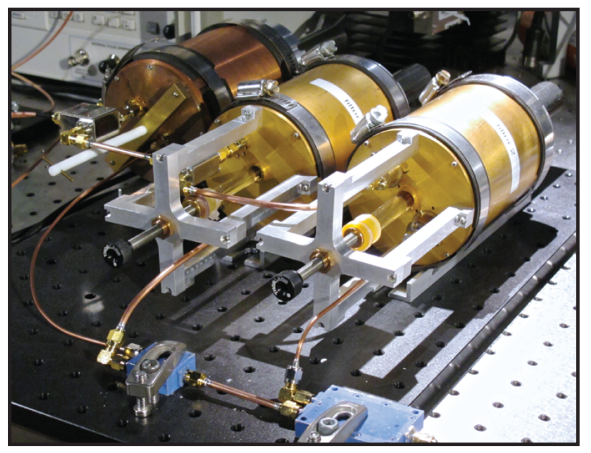

d)

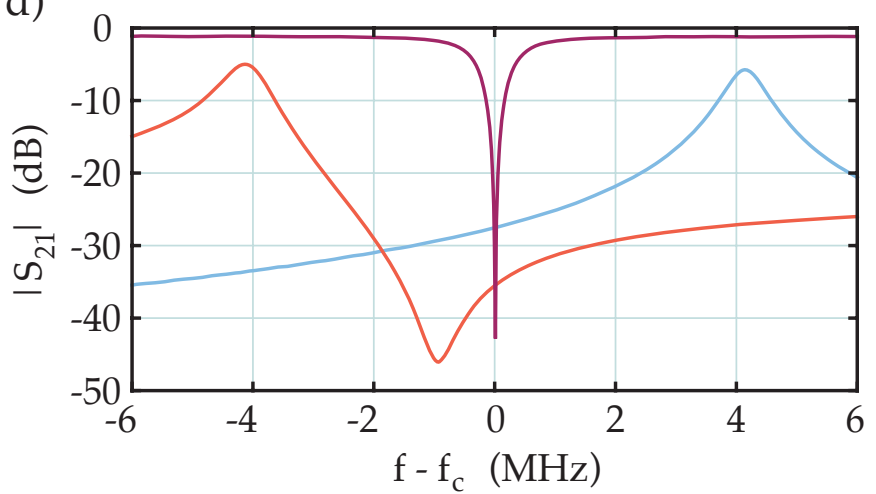

Figure 3.7: a) Photograph of copper filter cavity. The rough-tuning shaft at the back of the can adjusts the length of the cavity. The fine-tuning quartz rod at the front of the can can be inserted to adjust the frequency of the filter by $\sim 1 \mathrm{MHz}$. b) Schematics for operating the filter in transmission (left) or reflection (right). c) Filter cavity bank, set up in a combination of transmission and reflection. d) Example transmission through filter cavities operating in tranmission (red and blue) or reflection (purple). Note the anti-resonance in the red filter's transmission that adds attenuation near the cavity frequency.

filter the noise using a bank of adjustable cylindrical cavities (Fig. 3.7). The filter cavities have two ports, and thus can either be used as transmission, band-pass filters or the second port can be shorted, and the reflected signal can be picked off with a circulator for a band-reject filter.

Once the phase noise is dominated by room-temperature thermal noise for all relevant pump powers, it can be reduced by cold attenuators inside the fridge. If noise with temperature $T_{i n}$ is incident upon an attenuator with gain $G<1$ at temperature $T_{0}$, the resulting noise temperature at the output of the attenuator is

$$
T_{\text {out }}=T_{\text {in }} G+T_{0}(1-G) .
$$

For Device 1, we had cold attenuators with 10, 5, 8, and $16 \mathrm{~dB}$ attenuation placed on stages with temperatures of $4.2 \mathrm{~K}, 1.7 \mathrm{~K}, 100 \mathrm{mK}$, and $10 \mathrm{mK}$. For Device 2, we changed our input line, with one 


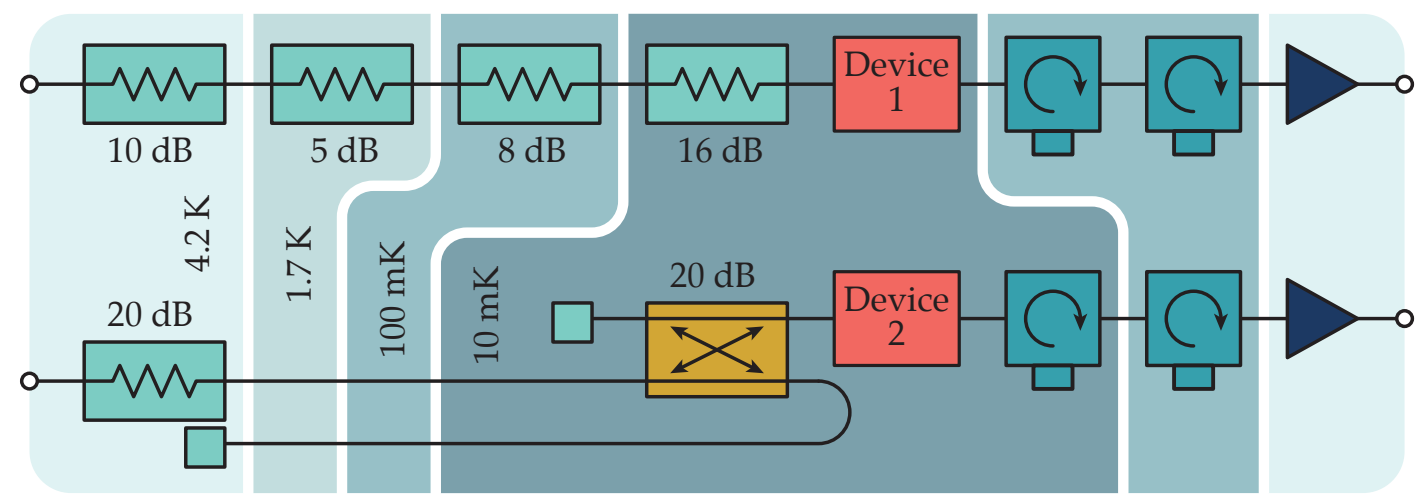

Figure 3.8: Comparison of the fridge input and output lines for Devices 1 and 2. Device 1's input line has a chain of attenuators, while Device 2's has a single $20 \mathrm{~dB}$ attenuator at $4 \mathrm{~K}$ and a directional coupler at the base temperature. Device 1's output line has both circulators on the cold stage, while Device 2's has one at base temperature.

$20 \mathrm{~dB}$ attenuator placed directly in the $4.2 \mathrm{~K}$ liquid helium bath, and one $20 \mathrm{~dB}$ directional coupler placed on the mixing stage. The coupler's directly-connected port is connected to a termination (i.e., a $50 \Omega$ load) at the $4 \mathrm{~K}$ plate, so the majority of the power going into the coupler is dissipated at a stage with much higher cooling power. The coupled port goes to the device, and the isolated port is connected to a termination at the mixing plate. When the mixing plate is at $10 \mathrm{mK}$, Device 1 thus has a temperature of $60 \mathrm{mK}$ at its left input port, and Device 2 has an input temperature of $80 \mathrm{mK}$. In terms of cavity quanta at $\omega_{c}$ for Device 1 or $2, n_{c, L}^{t h}$ is 0.013 or 0.025 , respectively. In practice, there is additional attenuation due to non-superconducting cables used above the $4 \mathrm{~K}$ plate, and additional reflection due to connectors and non-ideal components. Thus, the noise temperature of the input port is less than the theoretical value calculated here. Furthermore, as $n_{c}^{t h}=\Sigma_{i} \frac{\kappa_{i}}{\kappa} n_{c, i}^{t h}$, the occupation at the input port is reduced by a factor of $\kappa_{L} / \kappa$, which is between $1 / 5$ and $1 / 10$. Figure 3.8 shows the input line circuit diagrams for both Device 1 and Device 2.

\subsubsection{Cryogenic switches}

In order to test more than one device per cool-down while avoiding the heat load of running many cables from room temperature down to the mixing stage, we use two microwave switches on the mixing stage to switch the input and output lines between different devices. The switches are off-the-shelf Radiall R573423600 latching SP6T microwave switches with a 0-18 GHz operation bandwidth (Fig. 3.9). The switches are designed to work at room temperature, but can be operated at cryogenic temperatures. In order to reset the switch and latch to a new position, it is necessary to send current pulses to the switch. These pulses heat the mixing stage up to $\sim 100 \mathrm{mK}$. The fridge 


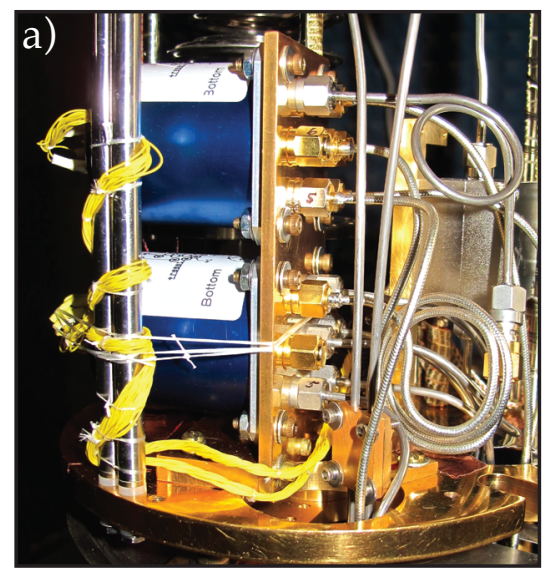

b)

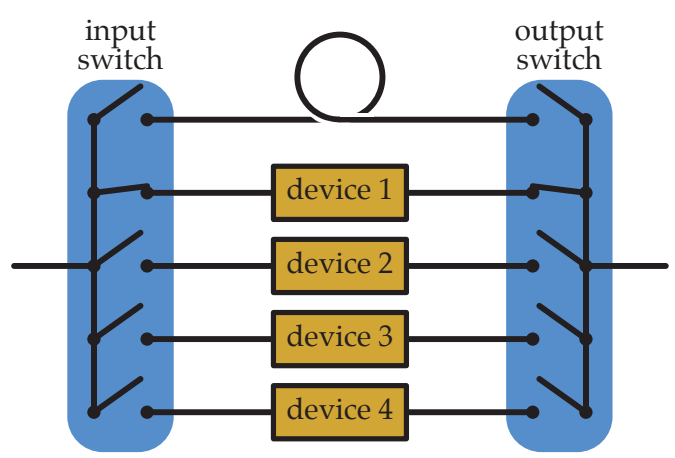

Figure 3.9: a) Two Radiall R573423600 switches (blue) mounted to the mixing stage. b) Schematic for switch positions. The first switch position is a section of cable with the same length as the cables that are connected to the devices. This channel is useful for calibrations and diagnostics.

then takes 30 minutes to cool back down to $20 \mathrm{mK}$. It is possible to modify the switch to use half the current [62], which would lead to less heating.

Although the switches have six positions, we use only five: four for devices, and one for a through. The cables on the through channel are the same length as the cables from the input switch to the devices and back to the output switch, so we can use the through channel to calibrate the fridge transmission if needed. The through channel is also useful for diagnosing any noise sources that we observe in the system.

\subsubsection{Output line: circulators and amplifiers}

To ensure that the output port does not see the $\sim 4 \mathrm{~K}$ noise temperature of the cryogenic amplifier, we place two circulators (Pamtech CTH4080) between the device and the amplifier. For Device 1, these circulators were both located on the cold plate. We found, however, that they contributed a measurable occupation of $n_{c, R}^{t h} \approx 0.3$ to the cavity occupation. For Device 2, one of the circulators was therefore moved to the mixing stage. The location of the circulators for the two devices is shown in Fig. 3.8.

As the circulators use magnetic material to break the symmetry between input and output ports, it is important that they have adequate magnetic shielding to prevent them from interfering with our superconducting devices. For Device 1, this shielding was provided by a sheet of mu-metal that we bent by hand in the lab. For Device 2, we had Pamtech install shielding on the circulators. Additionally, there is a cryoperm shield mounted to the cold stage and a cryoperm plate on the mixing stage just above the cold fingers that further shield the devices from the circulators.

After the circulators, we use a high electron mobility transistor (HEMT) amplifier to amplify 


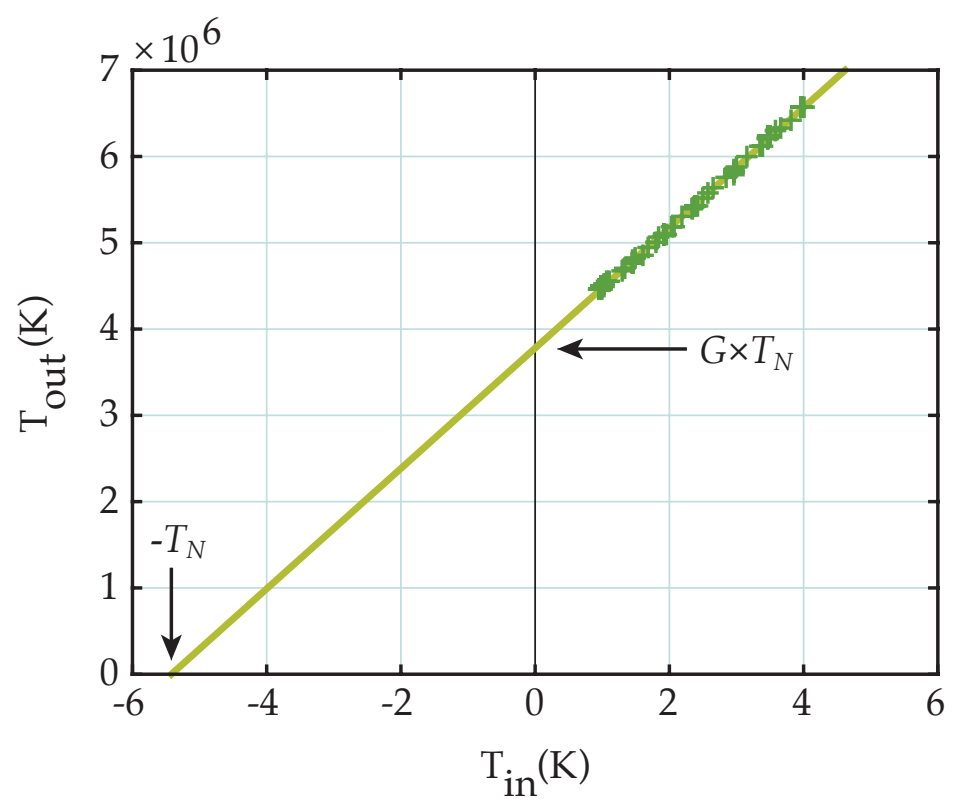

Figure 3.10: Measurement of the output line noise temperature and gain. The noise power of the system is measured at different temperatures, $T_{i n}$, between 1 and $4 \mathrm{~K}$ and converted to temperature via $P_{\text {out }} / \mathrm{RBW}=k_{B} T_{\text {out }}$ (dark green + symbols). As $T_{\text {out }}=\left(T_{\text {in }}+T_{N}\right) G$, the system noise temperature, $T_{N}$ can be found from the x-intercept of a linear fit to $T_{\text {out }}$ vs. $T_{\text {in }}$ (green line). The system gain can then be found from the y-intercept. We find $T_{N}=5.42 \pm 0.03 \mathrm{~K}$ and $G=58.4 \mathrm{~dB}$.

the signal from our device. The HEMT (model CIT-4254-077) was made at Caltech by the Weinreb group, and has an input noise temperature of $3.5 \mathrm{~K}$ and gain of $38 \mathrm{~dB}$ at $5 \mathrm{GHz}$. The HEMT has sufficient gain to dominate the noise of the output line that follows it. Due to $\sim 1-2 \mathrm{~dB}$ of losses between the device and the amplifier, however, we measure the effective noise temperature of our output line to be $5.42 \pm 0.03 \mathrm{~K}$ (Fig. 3.10).

At room temperature, a $120 \mathrm{~K}$ noise temperature MiTeq LCA 0408 amplifier provides $25 \mathrm{~dB}$ of additional amplification. The signal is then fed directly into an Agilent N9020A spectrum analyzer, which measures the symmetrized current spectral density, as discussed in Section 2.6.1. In general, we are interested in two types of spectrum analyzer measurements. We either measure the low-power noise spectral density of the cavity and mechanics, $S_{\text {out }}[\mathrm{dBm} / \mathrm{Hz}]$, or we measure the large pump through power, $P_{t h r u}[\mathrm{dBm}]$. If, instead of performing a spectral measurement, we need to measure the driven response of the device, we simply couple Port 1 of an Agilent N5230A or Agilent 8753ES vector network analyzer into the input line via a $10 \mathrm{~dB}$ directional coupler and connect the output line to Port 2 of the VNA rather than to the spectrum analyzer. 

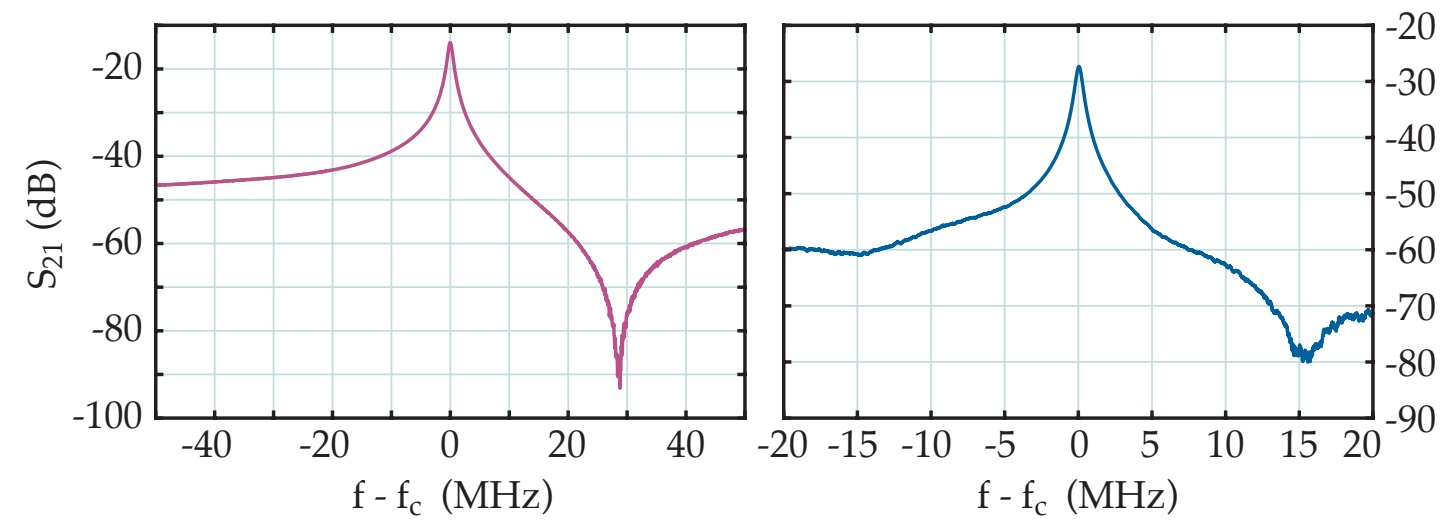

Figure 3.11: Microwave transmission for Device 1 (left) and Device 2 (right). The parasitic bypass channel creates the anti-resonance on the blue side of the cavity.

\subsection{Device characterization and calibrations}

\subsubsection{Parasitic channel}

When we measure the driven response of our devices over a frequency range much larger than the cavity linewidth, we don't see the expected Lorentzian response in the wings. Instead, we see an anti-resonance indicative of the presence of a parasitic bypass channel (Fig. 3.11). This bypass channel can be modeled as a reactance, $X_{\|}$, in parallel with the cavity, as described in the SI of $[60]^{1}$ and shown in Fig. 3.12a. With this model, we find that the driven response of the cavity is

$$
S_{21}(\omega)=-\frac{\sqrt{\kappa_{L} \kappa_{R}}-2 R_{L} X_{\|}^{-1}\left(\omega-\omega_{c}\right)}{\kappa / 2-i\left(\omega-\omega_{c}\right)}
$$

where $R_{L}=50 \Omega$ is the impedance at the input and output of the cavity. This transmission is shown in Fig. 3.12b. The gain in power of the output line is modified by a factor $\lambda(\omega)$ relative to the gain on cavity resonance:

$$
G(\omega)=\lambda(\omega) G\left(\omega_{c}\right)=\left|1-\frac{2 R_{L}}{X_{\|}} \frac{\left(\omega-\omega_{c}\right)}{\sqrt{\kappa_{L} \kappa_{R}}}\right|^{2} G\left(\omega_{c}\right)
$$

We find that the reactance is approximately $X_{\|} \sim 10 \mathrm{k} \Omega$. We can thus write $\lambda$ in the form:

$$
\lambda(\omega)=\left[1-\xi\left(\omega-\omega_{c}\right)\right]^{2}
$$

for some constant $\xi$.

\footnotetext{
${ }^{1}$ Here, I use the convention that the reactance is equal to $\operatorname{Im}[Z]$, whereas the SI of [60] defines the reactance as $i \operatorname{Im}[Z]$.
} 
a)

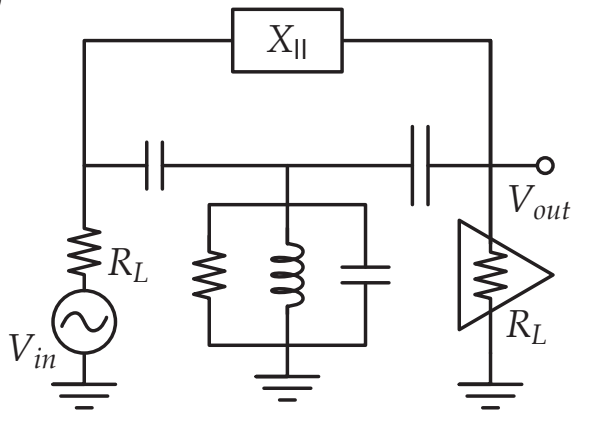

b)

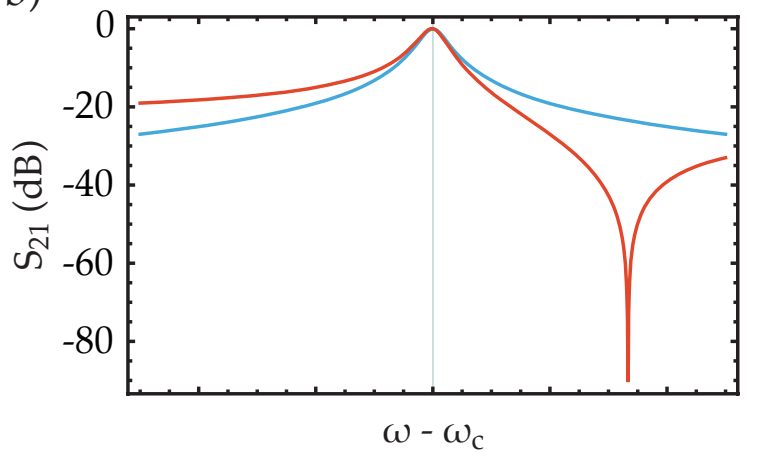

Figure 3.12: Bypass channel model. a) Circuit diagram for the model. The microwave cavity is depicted as an RLC circuit with input and output capacitors. b) Calculated transmission based on the model from (a). Lorentzian transmission is shown in blue; transmission with a bypass channel is shown in red.

\subsubsection{Thermal calibration}

To calibrate the measured noise spectrum at the output of our measurement chain against known thermal occupations, we follow a procedure similar to the one described in [25]. As described in Section 2.6.2, the output spectrum for the sideband of a red or blue-detuned tone in the weak-driving regime looks like a Lorentzian on top of a noise background. Taking into account the output line noise and gain, the sideband spectrum centered at $\omega_{c} \pm \delta$ becomes

$$
S_{m e c h}[\omega]-S_{0}=\hbar \omega_{c} G\left(\omega_{c}\right) \frac{\kappa_{R}}{\kappa} \frac{\gamma_{o p}^{ \pm} \gamma_{m}}{\left(\gamma_{m} / 2\right)^{2}+\omega^{2}} n_{m}^{t h}
$$

The integrated sideband power is then

$$
P_{m}^{ \pm}=\hbar \omega_{c} G\left(\omega_{c}\right) \kappa_{R} \frac{\gamma_{o p}^{ \pm}}{\kappa} n_{m}^{t h}
$$

The through power of the pumps is given by $P_{t h r u}^{ \pm}=\hbar \omega_{ \pm} G\left(\omega_{ \pm}\right) \kappa_{R} n_{p}^{ \pm}$, so the normalized sideband power can be written as

$$
\left(\frac{P_{m}}{P_{t h r u}}\right)_{ \pm}=\frac{\omega_{c} G\left(\omega_{c}\right)}{\omega_{ \pm} G\left(\omega_{ \pm}\right)}\left(\frac{2 g_{0}}{\kappa}\right)^{2} n_{m}^{t h}
$$

Note that, while the output gain is approximately flat on scales less than $1 \mathrm{MHz}$, it is not constant with frequency. In particular, the parasitic bypass channel described in Section 3.4 .1 creates a gain of $\lambda_{ \pm}=\left(1 \mp \xi \omega_{m}\right)^{2}$ at $\omega_{ \pm}$relative to that at $\omega_{c}$.

As the mechanical occupation follows a Bose-Einstein distribution, at the high occupations of the thermal cal $\left(n_{m}^{t h} \geq 100\right)$, the thermal occupation is linear in temperature: $n_{m}^{t h}=k_{B} T / \hbar \omega_{m}$. We 
thus find that the normalized sideband power is proportional to the fridge temperature:

$$
\left(\frac{P_{m}}{P_{\text {thru }}}\right)_{ \pm}=\frac{\omega_{c}}{\omega_{ \pm} \lambda_{ \pm}}\left(\frac{2 g_{0}}{\kappa}\right)^{2} \frac{k_{B}}{\hbar \omega_{m}} T
$$

If we measure $P_{m} / P_{t h r u}$ vs. temperature for both the red- and blue-detuned tones, the two slopes allow us to find both $g_{0}$ and $\lambda_{ \pm}$, as all other quantities in Eq. 3.8 are known or measureable. Plugging these values back into Eq. 3.7 also allows us to directly relate any measured normalized sideband area to the number of mechanical quanta.

\subsubsection{Linewidth broadening and pump power calibration}

As seen in Section 2.8.2, a single, red-detuned tone causes the mechanical linewidth to broaden by an amount proportional to $G_{-}^{2}$ :

$$
\gamma_{t o t}=\gamma_{m}+4 G_{-}^{2} / \kappa
$$

$G_{-}^{2}=g_{0}^{2} n_{p}$, so $G_{-}^{2}$ is proportional to $P_{t h r u}^{-}$. By measuring the mechanical linewidth via driven response at different applied powers, $G_{-}^{2}$ can be calibrated to the measured output pump power through some constant of proportionality: $G_{-}^{2}=\beta_{-} \times P_{t h r u}^{-}$.

For some devices, $\kappa$ changes appreciably with pump power. We thus first find $\kappa$ with a fullcavity driven response for each applied power. We then find a linear fit to $\gamma_{\text {tot }}$ vs. $P_{t h r u}^{-} \bar{\kappa} / \kappa$, where $\bar{\kappa}$ is the mean value of all measured cavity linewidths. The obtained offset is then $\gamma_{m}$, and the obtained slope is $4 \beta_{-} / \bar{\kappa}$. As this calibration only involves driven responses and measurements of large through powers, it is more precise than the thermal calibration, which relies on low-SNR spectral measurements.

$G_{+}^{2}$ can also be calibrated from linewidth narrowing. For the data shown here, however, we calibrate $G_{+}^{2}$ by multiplying $\beta_{-}$by the balancing ratio found in the thermal calibration (i.e., the ratio of gains at $\omega=\omega_{ \pm}$). The calibration for $G_{+}^{2}$ thus has a greater uncertainty than that for $G_{-}^{2}$.

Once we know $g_{0}$ from the thermal calibration, we can also obtain a calibration for the number of pump photons versus through power:

$$
n_{p}^{ \pm}=\beta_{ \pm} P_{t h r u}^{ \pm} / g_{0}^{2}
$$




\section{Chapter 4}

\section{Device 1: Back-action evading measurements for direct detection of squeezed motion}

\subsection{Overview}

The first device (D1) in which we implemented the KMC squeezing scheme was the same device used in the back-action evasion experiments discussed in [51]. In this experiment, we successfully avoided both the quantum and classical measurement back-action while measuring a single quadrature of the mechanical motion. The results of the BAE experiment are summarized in Fig. 4.1. The relevant parameters for this device are shown in Table 4.1.

\begin{tabular}{cc} 
Parameter & Value \\
\hline$\omega_{m}$ & $2 \pi \times 4.0 \mathrm{MHz}$ \\
$\gamma_{m}$ & $2 \pi \times 10 \mathrm{~Hz}$ \\
$\omega_{c}$ & $2 \pi \times 5.45 \mathrm{GHz}$ \\
$\kappa$ & $2 \pi \times 860 \mathrm{kHz}$ \\
$g_{0}$ & $2 \pi \times 15.5 \mathrm{~Hz}$ \\
$x_{z p}$ & $\sim 1.8 \mathrm{fm}$ \\
\hline
\end{tabular}

Table 4.1: Parameters for Device 1.

As part of the BAE experiment, a pair of strong pump tones perform a BAE measurement of $\hat{X}_{1}$, and a pair of weak probe tones perform a phase-dependent measurement of the mechanics to detect the back-action in $\hat{X}_{2}$ (Fig. 4.1c,d). This measurement scheme is easily modified for our squeezing experiment; the strong BAE pump tones are simply replaced by strong squeezing tones. As long as the probe tones are weaker than the pump tones and the pump and probe sidebands are separated by many linewidths, the presence of the probe tones should not affect the squeezed state. As squeezing produces excess damping, to separate the probe tone sideband from the pump tone sideband, it is necessary to detune the probe sideband by up to $\sim \kappa / 10$ from the cavity center (Fig. 4.2). Extra 
a)

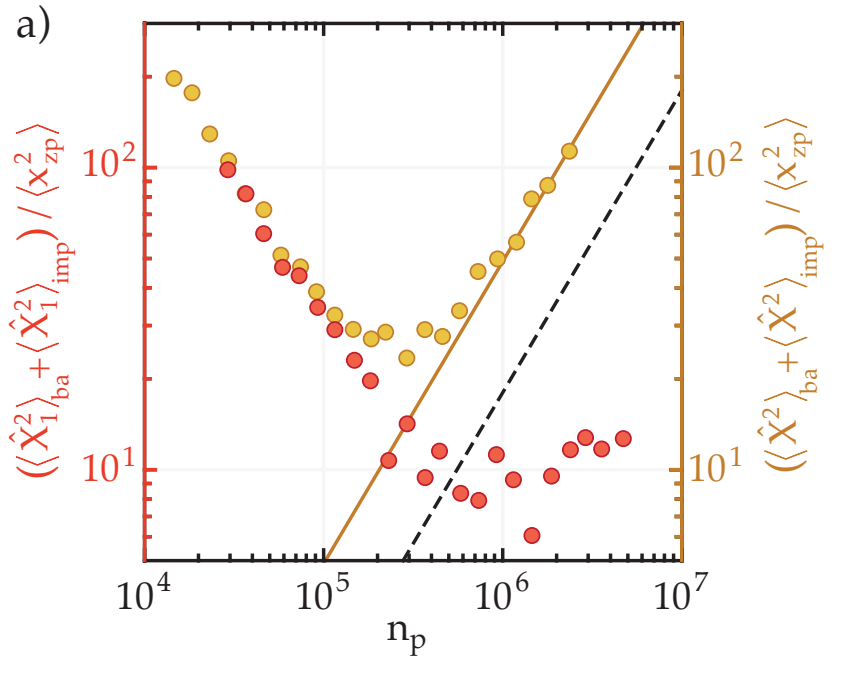

b)
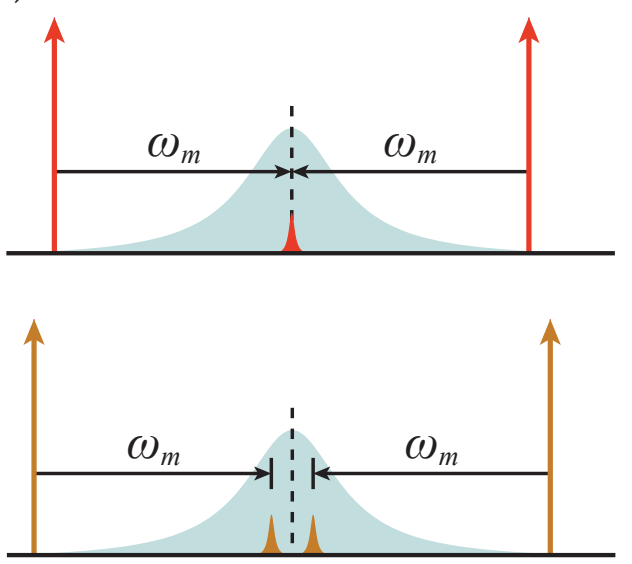

c)

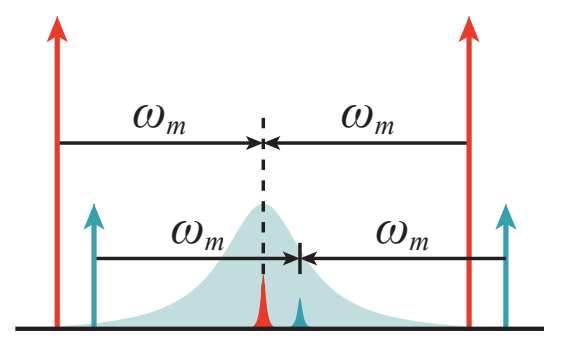

d)

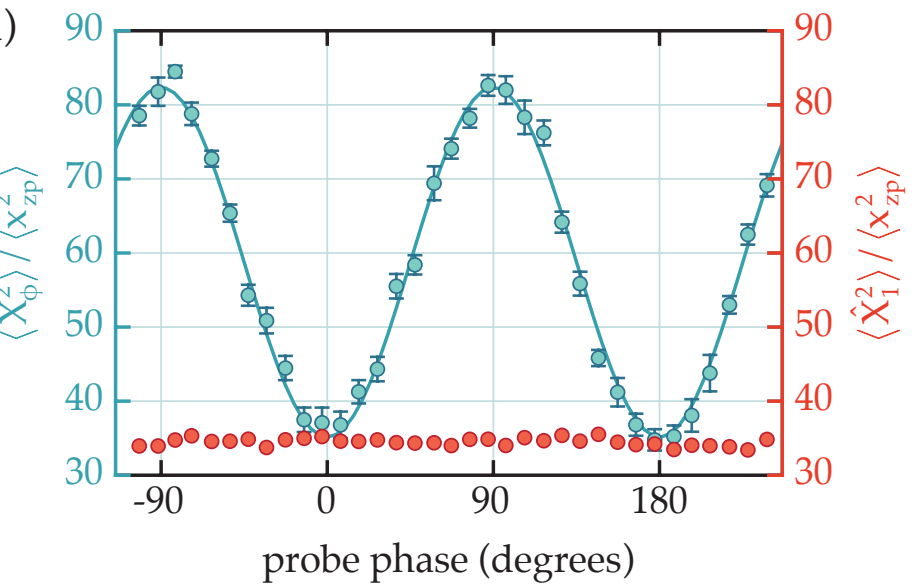

Figure 4.1: a) Total imprecision and back-action noise for two tones in a BAE configuration (red) and in a detuned configuration (yellow). Configurations are shown in (b). The solid yellow line is a fit to the detuned tones' back-action, while the dashed black line is the quantum back-action. The BAE tones avoid both the classical and quantum back-action at high pump powers. c) BAE pump and probe configuration. The BAE pumps (red) measure the $\hat{X}_{1}$ quadrature fluctuations and add noise to $\hat{X}_{2}$. The relative phase of the probes (turquoise) can be adjusted so that they can measure fluctuations in a quadrature oriented with phase $\phi$ with respect to $\hat{X}_{1}$. The probe tones are weaker than the BAE pumps so that they don't add significant back-action to $\hat{X}_{1}$ for any $\phi$. d) Fluctuations measured by the BAE pumps (red) and BAE probes (turquoise) as a function of probe phase. For all phases, the BAE pumps measure $\left\langle\hat{X}_{1}^{2}\right\rangle$, while the probes measure $\left\langle\hat{X}_{1}^{2}\right\rangle$ at $0^{\circ}$ and $180^{\circ}$, and $\left\langle\hat{X}_{2}^{2}\right\rangle$ at $\pm 90^{\circ}$. 


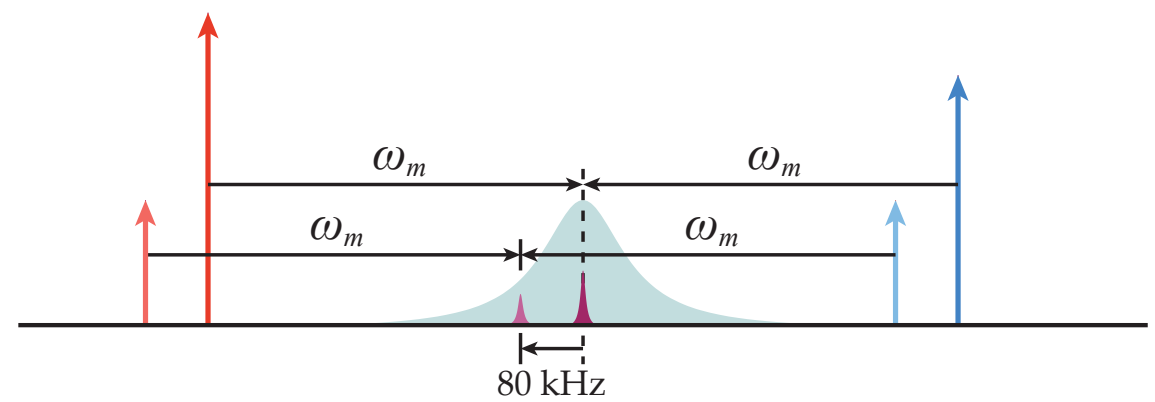

Figure 4.2: Pump scheme for BAE measurements of the squeezed state. The squeezing tones are shown in dark red and blue, while the BAE probes are shown in light red and blue. The measured signal is the sideband of the BAE probe tones at $\omega_{c}-(2 \pi \times 80 \mathrm{kHz})$.

care must therefore be taken to properly calibrate the probe sideband area. Moreover, since there are squashing (anti-squashing) effects associated with excess red (blue) probe tone power, it is important to have an accurate way to balance the red and blue probe powers in the presence of the large squeezing tones. This chapter explains these calibrations and demonstrates a phase-dependent measurement of a squeezed mechanical state with minimum fluctuations of $1.09 \pm 0.06 x_{z p}^{2}$.

\subsection{Device calibrations and characterizations}

\subsubsection{Thermal calibration}

For thermal calibration, weak red- and blue-detuned pumps are applied so that their sidebands are detuned by many linewidths, as described in Section 3.4.2. The pump powers are chosen to be $\sim 1100$ photons. At this occupation, $\gamma_{o p} \sim 1 \mathrm{~Hz}$ for each pump, so a power imbalance of $10 \%$ between the two pumps would result in damping or amplification of less than $1 \%$, given the mechanics' $10 \mathrm{~Hz}$ linewidth. At these pump powers, we also expect insignificant heating of the mechanical and cavity baths.

As the signal that we will want to measure for this device is a BAE probe sideband detuned by $\Delta / 2 \pi=-80 \mathrm{kHz}$ from the cavity center, we perform the thermal calibration with the sidebands centered at $\omega_{c}-2 \pi \times 80 \mathrm{kHz}$ and detuned from each other by $1 \mathrm{kHz}$, as seen in Fig. 4.3. With a non-zero $\Delta$, the optomechanical damping (and thus the sideband transduction) for both pumps is reduced by a factor of $1 /\left(1+(2 \Delta / \kappa)^{2}\right)$, or by about $3.3 \%$, relative to sidebands centered on the cavity frequency. By performing the calibration at $\omega_{c}+\Delta$, this correction is automatically included in the thermal calibration.

Fig. 4.4 shows the results of the thermal calibration for this device. From the fits to the scaled 


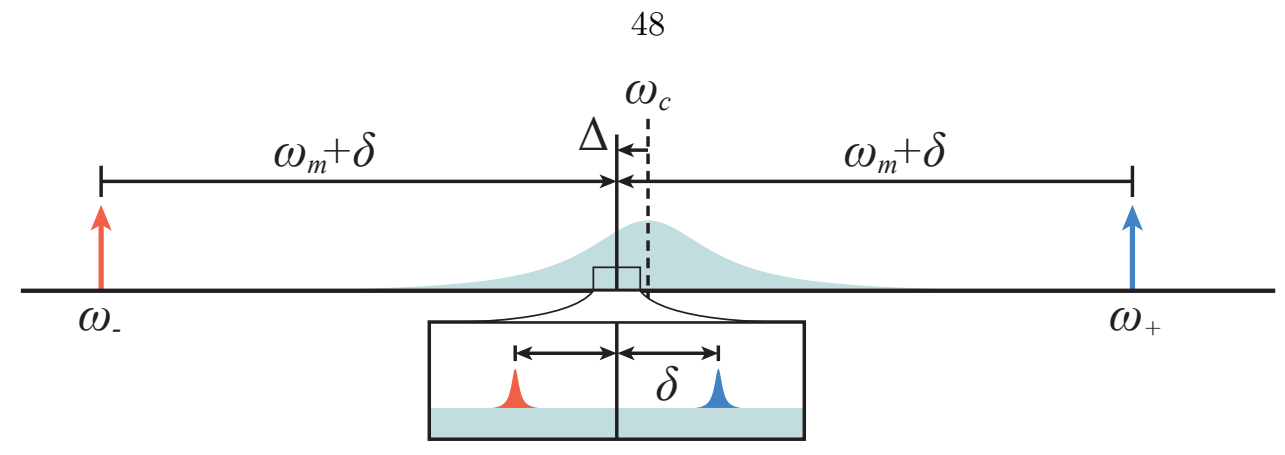

Figure 4.3: Pump and sideband scheme for Device 1's thermal calibration. Here, $\Delta=-2 \pi \times 80$ $\mathrm{kHz}$, and $\delta=2 \pi \times 500 \mathrm{~Hz}$.

sideband power vs. temperature, we obtain

$$
\begin{aligned}
& \left(\frac{P_{m}}{P_{t h r u}}\right)_{-}=(4.995 \pm 0.107) \times 10^{-6} \times T \\
& \left(\frac{P_{m}}{P_{t h r u}}\right)_{+}=(8.848 \pm 0.189) \times 10^{-6} \times T
\end{aligned}
$$

To find the parasitic channel parameter $\xi$, we solve

$$
\frac{\text { slope }_{+}}{\text {slope }_{-}}=\frac{\left(1-\xi\left(\Delta-\omega_{m}\right)\right)^{2}}{\left(1-\xi\left(\Delta+\omega_{m}\right)\right)^{2}}
$$

to find $\xi=(0.142 \pm 0.007) \omega_{m}^{-1}$. The coupling rate for this device is then $g_{0}=2 \pi \times(15.56 \pm 0.19 \mathrm{~Hz})$. From the parasitic channel parameter, we expect the probes to be balanced when $P_{t h r u}^{+} / P_{t h r u}^{-}=$ $-2.48 \pm 0.13$. From the slopes, we are also able to directly relate sideband area to occupational quanta:

$$
\begin{aligned}
\bar{n}_{\text {meas }} & =(10.411 \pm 0.223) \times 10^{9} \times\left(\frac{P_{m}}{P_{\text {thru }}}\right)_{-} \\
& =(5.877 \pm 0.126) \times 10^{9} \times\left(\frac{P_{m}}{P_{\text {thru }}}\right)_{+} .
\end{aligned}
$$

We use Eq. 4.1 to convert sideband area to units of mechanical quanta in our BAE probe measurements. If we'd like to calculate $\left\langle\hat{x}^{2}\right\rangle$ in units of $x_{z p}^{2}$ instead of in units of quanta, we can simply multiply the noise in quanta by 2 , as $x_{z p}^{2}$ corresponds to $1 / 2$ quantum:

$$
\begin{aligned}
\left\langle\hat{x}^{2}\right\rangle_{\text {meas }} & =(20.822 \pm 0.446) \times 10^{9} \times\left(\frac{P_{m}}{P_{\text {thru }}}\right)_{-} \\
& =(11.754 \pm 0.252) \times 10^{9} \times\left(\frac{P_{m}}{P_{\text {thru }}}\right)_{+} .
\end{aligned}
$$

Fig. 4.5 shows how the mechanical and cavity frequencies change with temperature. The me- 

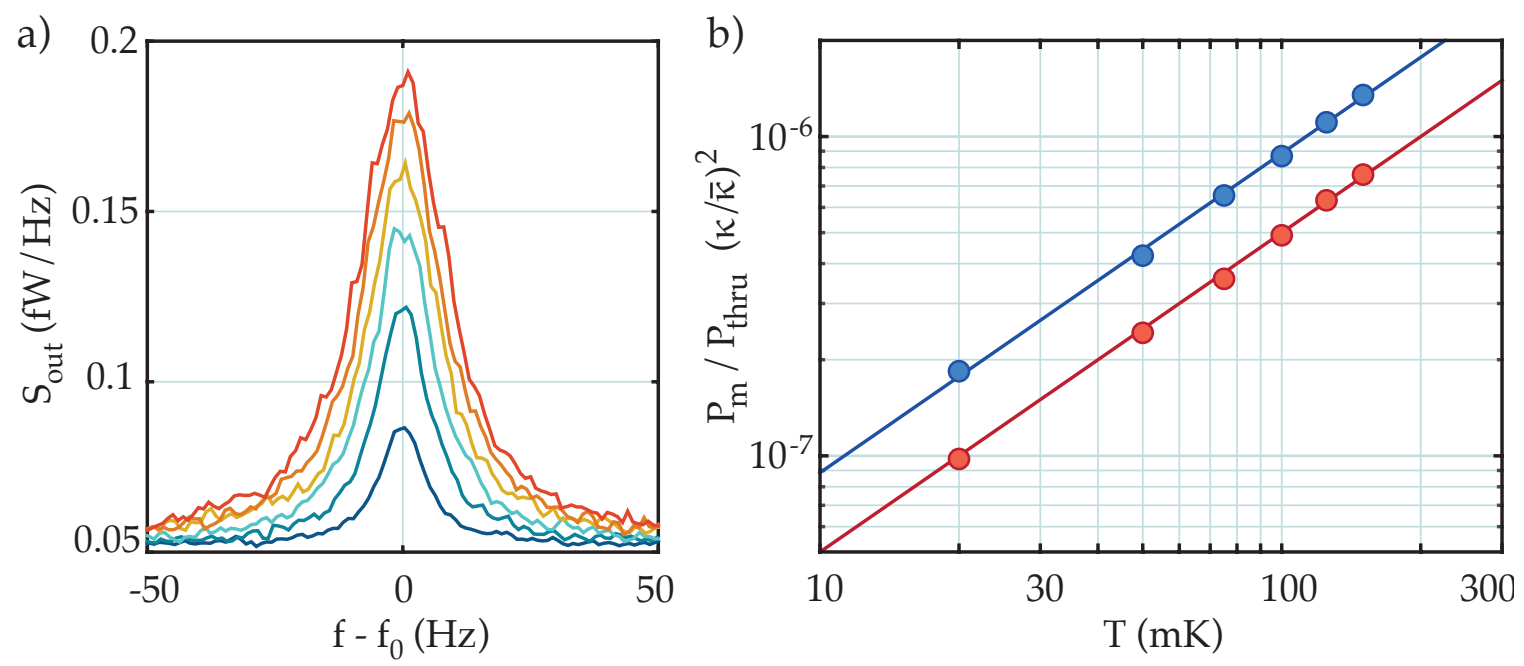

Figure 4.4: a) Red-detuned pump sideband noise spectra for temperatures ranging from $20 \mathrm{mK}$ (blue) to $150 \mathrm{mK}$ (red). b) Normalized sideband power versus temperature for both red- and blue-detuned pumps. The difference in output gain for the two pump frequencies is evident in the difference in the slopes.

chanical values are obtained by fits to the sideband spectra, while the cavity values are obtained from driven responses. The mechanical linewidth is observed to broaden with temperature, and the mechanical frequency to increase slightly. Note that all data were taken in the presence of the two weak pumps, so the cavity linewidth and frequency may not exhibit the same behavior as they would in the absence of any applied power. For these measurements, $\kappa$ changes by less than $1 \%$.

\subsubsection{Linewidth broadening}

We apply a single, red-detuned tone at $\omega_{-}=\omega_{c}-\omega_{m}$ and measure the resulting damping of the mechanical linewidth via driven response, as described in Section 3.4.3. Examples of driven response spectra are shown in Fig. 4.6b. By fitting $\gamma_{t o t}=\gamma_{m}+\gamma_{o p}$ to the linewidth vs. measured pump through power, we obtain

$$
\gamma_{t o t} / 2 \pi=(10.54 \pm 0.12) \mathrm{Hz}+(1.727 \pm 0.004) \times 10^{8}(\mathrm{~Hz} / \mathrm{W}) \times P_{\text {thru }}^{-},
$$

as seen in Fig. 4.6a. From this fit and the value of $g_{0}$ obtained in the thermal calibration, we find that $n_{p}^{-}=(1.541 \pm 0.038) \times 10^{11} \times P_{t h r u}^{-}$. Later changes to the output line of the measurement chain made it necessary to adjust the through power values by $0.76 \mathrm{~dB}$. For the squeezing data shown 
mechanics
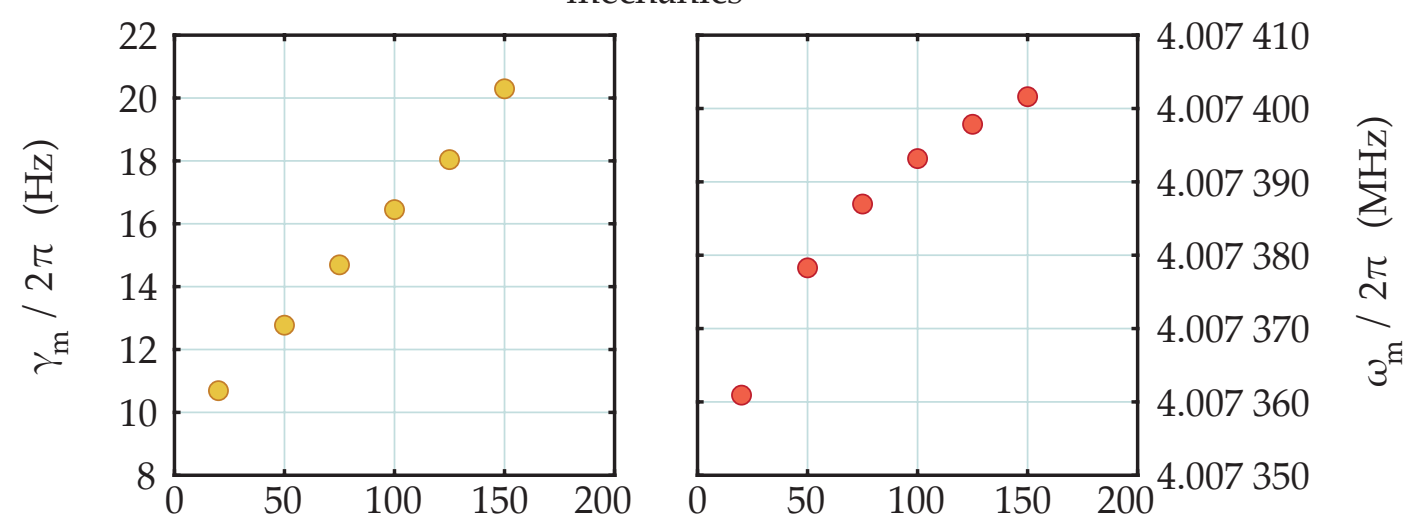

cavity
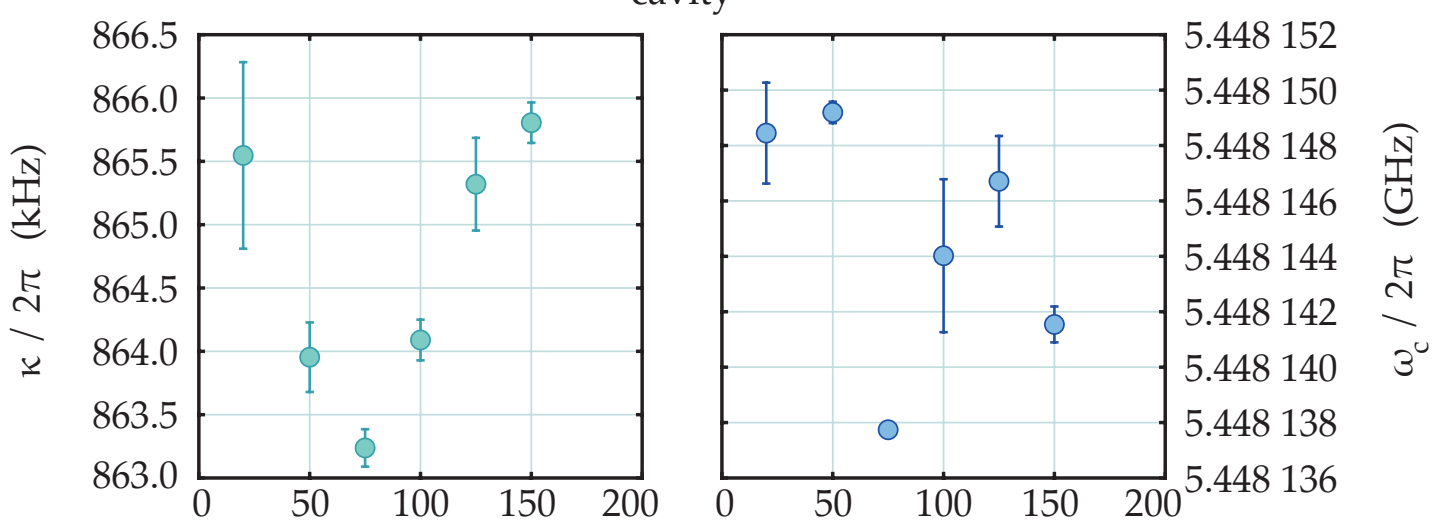

$\mathrm{T}(\mathrm{mK})$

Figure 4.5: Mechanical and cavity frequencies and linewidths as a function of temperature in the presence of two weak pumps. 


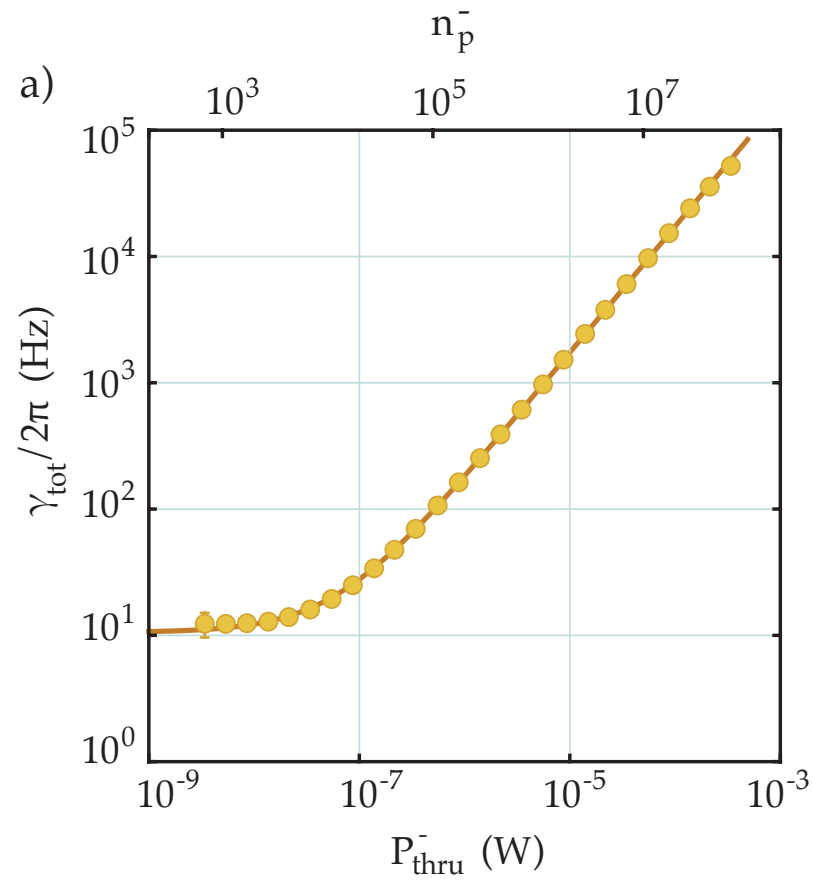

b)

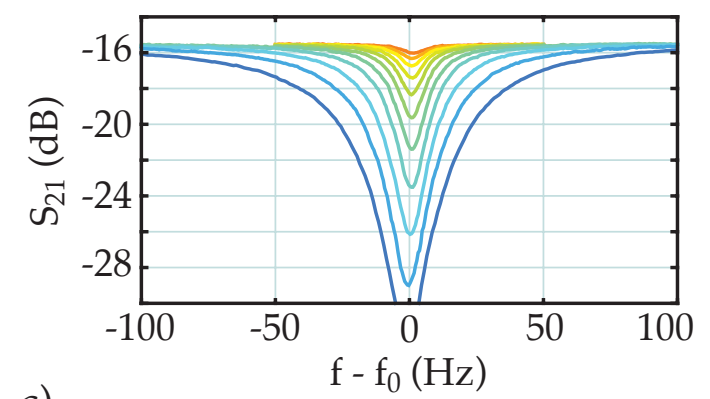

c)

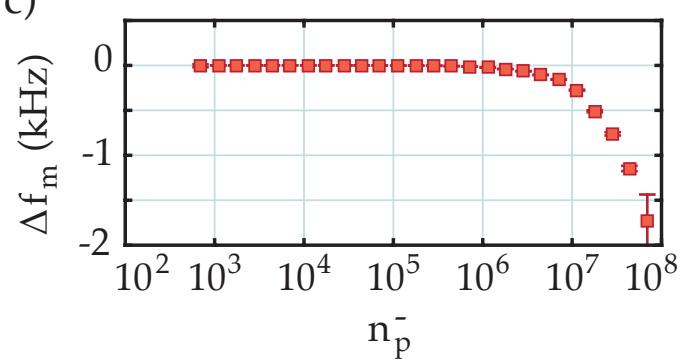

Figure 4.6: Linewidth broadening calibration. a) Mechanical linewidth vs. through power (yellow circles). The solid line is a fit to the first 17 data points: $\gamma_{t o t}=(10.54 \pm 0.12)+(1.727 \pm 0.004) \times$ $10^{8} \times P_{t h r u}^{-}$Hz. The upper axis indicates the corresponding pump occupation obtained from this calibration. b) Example driven responses for the first 10 pump powers, with the lowest power in red and the highest in dark blue. Here, $f_{0}$ indicates the sideband center frequency. The linewidths in (a) are obtained from Lorentzian fits to the complex transmission. c) Mechanical frequency vs. applied pump power.

later, we have

$$
\begin{aligned}
n_{p}^{-} & =(1.295 \pm 0.042) \times 10^{11} \times P_{t h r u}^{-} \\
n_{p}^{+} & =\frac{\left(1-\xi\left(\Delta-\omega_{m}\right)\right)^{2}}{\left(1-\xi\left(\Delta+\omega_{m}\right)\right)^{2}}(1.295 \pm 0.042) \times 10^{11} \times P_{t h r u}^{+} \\
& =(2.294 \pm 0.102) \times 10^{11} \times P_{t h r u}^{+},
\end{aligned}
$$

where $P_{t h r u}$ is in Watts.

\subsubsection{Balancing calibration}

For BAE probe measurements, it is very important that the probe tones have exactly the same intra-cavity power. If there is excess red or blue pump power, then there will be squashing or antisquashing present in the measured noise sideband due to the non-zero cavity bath occupation, and the quadrature noise will be either underestimated or overestimated, as described in Section 2.7.2. The thermal calibration tells us the ratio of the gains at the two probe frequencies, and thus lets us 
a)

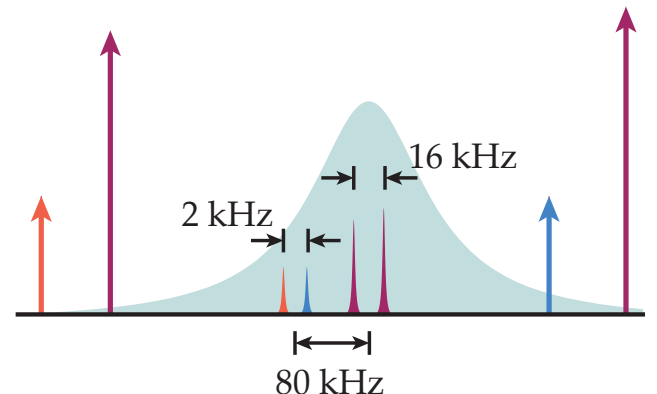

b)

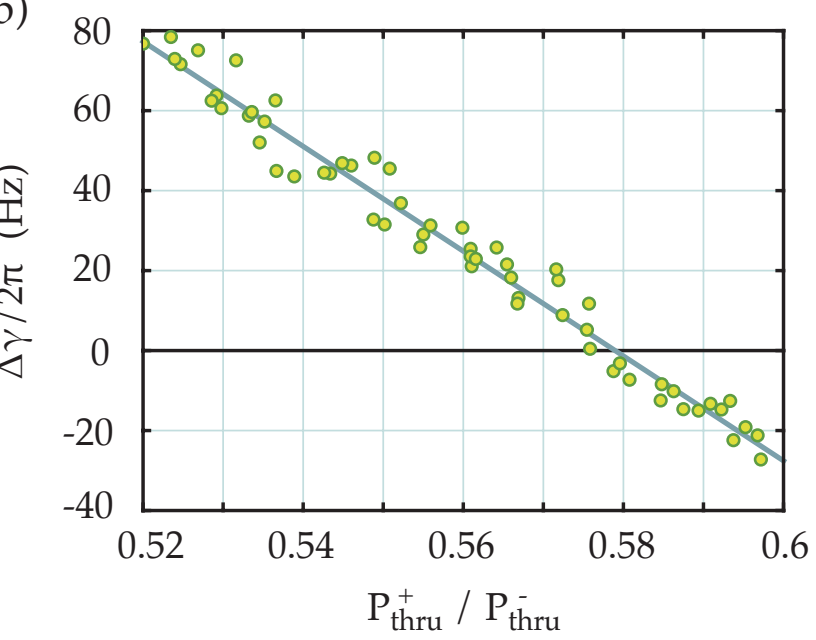

Figure 4.7: Balancing calibration. a) Frequency schematic for the balancing calibration. The two strong squeezing tones (purple) are adjusted so that the mechanical linewidth is $160 \mathrm{~Hz}$ and detuned to avoid parametric instabilities. The two probe tones (red and blue) are centered at the same $\Delta$ as during the squeezing measurements, but are also detuned slightly. b) Linewidth shift measured from fits to the red-detuned squeezing pump tone's spectral sideband at different blue probe powers.

know what the ratio of output probe powers necessary for balancing the probes. This calibration has a large error bar of $\pm 0.13 \mathrm{~dB}$, however, and is not accurate enough for our purposes. We thus perform a separate balancing calibration to find the output power ratio that produces equal red and blue probe occupations in the cavity.

In order for the conditions during balancing to be as similar as possible to the conditions during squeezing measurements, we adopt the following balancing procedure. First, we apply the squeezing tones, but increase the blue power and decrease the red power so that the mechanical linewidth is $160 \mathrm{~Hz}$. This narrow linewidth makes it easy to measure changes in the mechanical linewidth. We also detune the squeezing tones by $\delta=2 \pi \times 8 \mathrm{kHz}$ to avoid any parametric instabilities. We then apply the two BAE probe tones with $\Delta=-2 \pi \times 80 \mathrm{kHz}$ and $\delta=2 \pi \times 1 \mathrm{kHz}$. This pump configuration is shown in Fig. 4.7a. We measure the red-detuned squeezing pump's spectral sideband and the two probe tones' through powers with the probe tones on, and then we turn off the probe tones and measure the red squeezing pump's sideband again. The change in mechanical linewidth due to the presence of the probe tones tells us about the damping or anti-damping added by the probe tones. We repeat this procedure for different blue probe tone powers, keeping all other powers fixed. The results are shown in Fig. 4.7b. When the change in the mechanical linewidth is 0 , then the probe tones are balanced. From a fit to the data shown, this 0 intercept occurs when $P_{\text {thru }}^{+} / P_{\text {thru }}^{-}=-2.38 \pm 0.01 \mathrm{~dB}$. For our squeezing measurements, we ensure that our probe through powers are within $0.02 \mathrm{~dB}$ of this ratio. 


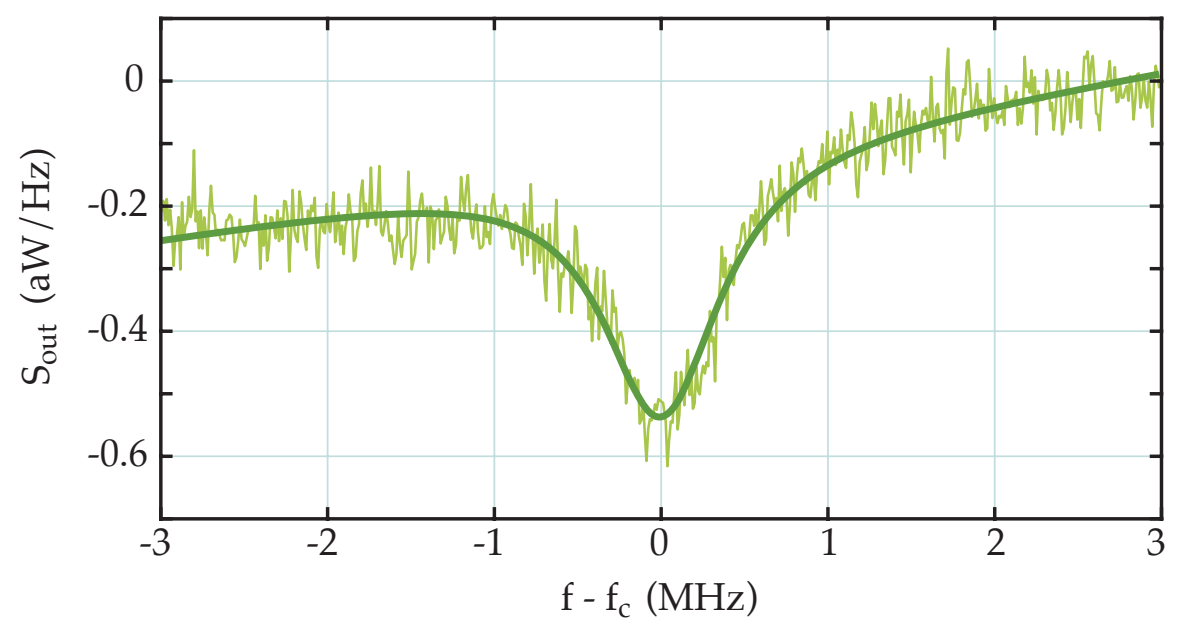

Figure 4.8: Measurement of $n_{c, R}^{t h}$. Light green spectrum is the cavity spectrum in the absence of pumps, minus the no-pump spectrum taken with the switch set to the through channel. Dark green line is a Lorentzian fit with a linear background. The fit yields an occupation of $n_{c, R}^{t h} \approx 0.37$.

\subsection{4 $n_{c, R}^{t h}$ measurements}

From Eq. 2.80, if there is excess microwave occupation at the right port, we should see a dip in the cavity spectrum when no pumps are applied. As seen in Fig. 4.8, we observe such a dip for D1. To calibrate the occupation due to this dip, we take spectra in the presence of no pumps both with the device connected, and then with the circuit switched to a through channel (Section 3.3.2). Subtracting off the floor from the through channel produces the spectrum shown in Fig. 4.8. We then integrate the Lorentzian area, $A_{c}$, and use the $n_{p}$ calibration from Section 4.2.2 to convert this area to a cavity occupation. We must also take into account the difference in gain at $\omega_{-}$and $\omega_{c}$ due to the parallel bypass channel, as described in Section 4.2.1. As shown in Eq. 2.80, there is an additional factor of $\left(1-\kappa_{R} / \kappa\right)$ that enters into the spectrum, where $\kappa_{R} \approx 2 \pi \times 450 \mathrm{kHz}$ for this device. The occupation is thus:

$$
n_{c, R}^{t h}=\frac{A_{c} \times \beta_{-}}{\left(1-\kappa_{R} / \kappa\right)} \frac{G\left(\omega_{-}\right)}{G\left(\omega_{c}\right)} .
$$

Using this method, we find that $n_{c, R}^{t h}$ is approximately 0.37 quanta, which corresponds to a bath temperature of $\sim 200 \mathrm{mK}$. This observation led us to change the location of the circulators on the output line before measuring other devices, as described in Section 3.3.3.

\subsubsection{Cooling estimates}

To characterize the performance of the device, we perform a sideband cooling experiment by applying a single tone at $\omega_{c}-\omega_{m}$ and measuring the resulting mechanical occupation vs. applied power. As 


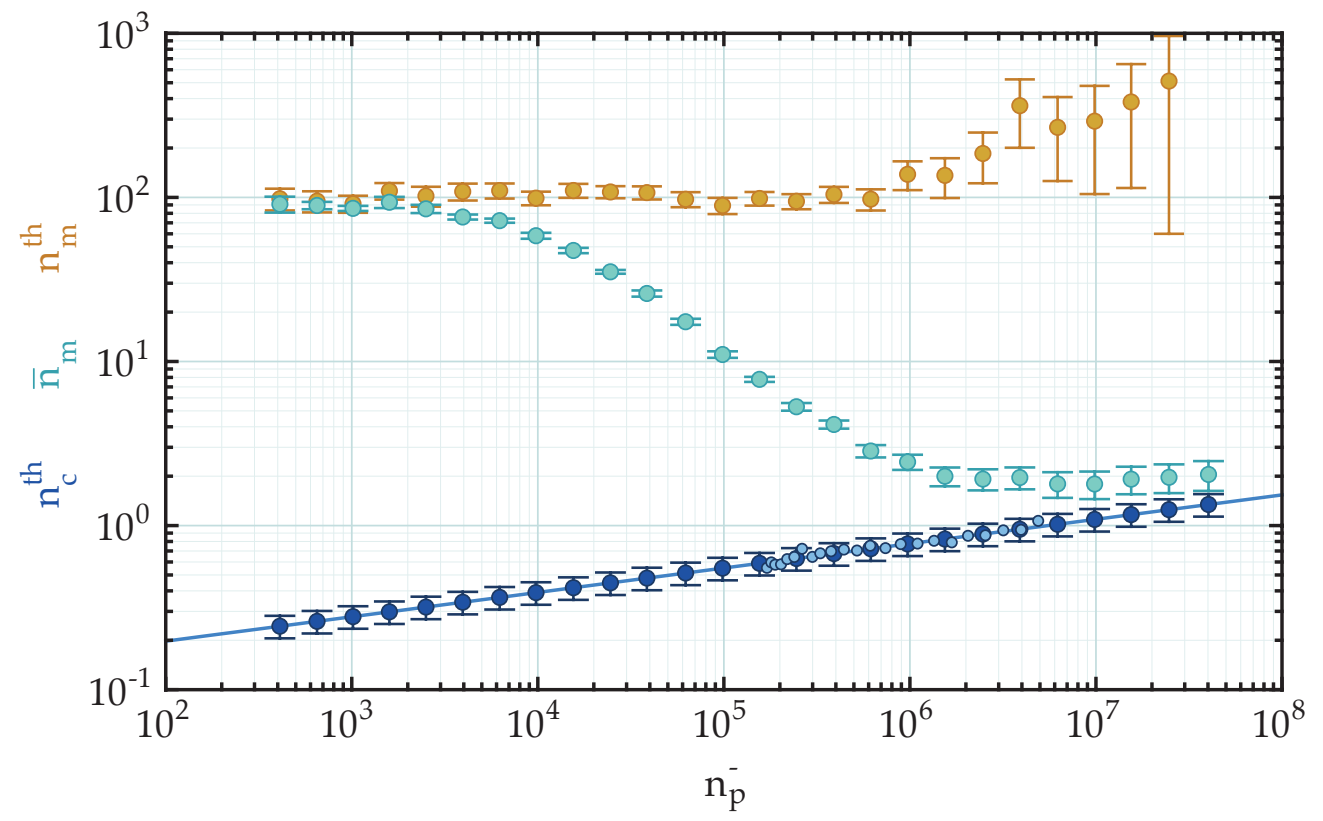

Figure 4.9: Cooling estimates for D1. The measured $n_{c}^{\text {th }}$ for two pumps in a BAE configuration is shown in small, light blue circles. Solid blue line indicates a power-law fit to these data. Dark blue circles are corresponding $n_{c}^{t h}$ estimates calculated from the power law, with error. $\bar{n}_{m}$ calculated from $\bar{n}_{\text {meas }}+2 n_{c}^{t h}-n_{c, R}^{t h}$ is shown in turquoise. The mechanical occupation is limited to about 1-2 quanta at high pump powers. $n_{m}^{\text {th }}$ inferred from $\bar{n}_{m}$ is shown in yellow. Some heating of the thermal bath is evident above $n_{p}^{-}=10^{6}$.

the sideband power is proportional to $\bar{n}_{m}-\left(2 n_{c}^{t h}-n_{c, R}^{t h}\right)$ (see Eq. 2.78), we need some way to measure $n_{c}^{t h}$ vs. applied power in order to extract the mechanical occupation. At the time of the cooling measurements presented in this section, we had yet to establish a robust way to measure $n_{c}^{\text {th }}$, which is made more difficult when $n_{c, R}^{t h} \neq 0$. As our goal at the time was to perform BAE measurements, we did have a set of measurements of cavity spectra vs. applied BAE power, with corresponding backgrounds taken with the microwave switch set to the through channel. These measurements are not taken at the same total powers as the cooling measurements, but we can extract $n_{c}^{\text {th }}$ vs. total BAE power $\left(n_{p}^{-}+n_{p}^{+}+n_{p}^{\text {cool }}\right.$, where $n_{p}^{\text {cool }}$ is the occupation of a cooling tone used during BAE measurements to broaden the mechanical linewidth). We then fit $n_{c}^{\text {th }}$ vs. total pump occupation with a power law and use this model plus the $n_{c, R}^{t h}$ occupation obtained in the previous section to estimate the mechanical occupation. The results of this measurement are shown in Fig. 4.9.

With the given estimates for $n_{c}^{t h}$, we find that the mechanical occupation reaches between 1 and 2 quanta before starting to heat up. Later, in Appendix B, we'll see that the assumption that the cavity occupation in the presence of a single, red-detuned pump is the same as the occupation in the presence of two, equal-powered red- and blue-detuned pumps with the same total power is not necessarily valid. It is thus likely that we have over-estimated $n_{c}^{t h}$ here, which would also lead us to 
over-estimate $\bar{n}_{m}$ and $n_{m}^{\text {th }}$ in Fig. 4.9 .

\subsection{BAE probe measurements}

\subsubsection{Measurement set-up}

As $\hat{X}_{1}$ and $\hat{X}_{2}$ represent the amplitudes of the $\cos \omega_{m} t$ and $\sin \omega_{m} t$ components of $\hat{x}$, they define a set of axes rotating at $\omega_{m}$. The phase of the $\hat{X}_{1}$ quadrature is set by the phase difference between the two squeezing pump tones:

$$
\cos \left(\left(\omega_{c}-\omega_{m}\right) t-\phi_{0}\right)+\cos \left(\left(\omega_{c}+\omega_{m}\right) t+\phi_{0}\right)=2 \cos \left(\omega_{c} t\right) \cos \left(\omega_{m} t+\phi_{0}\right)
$$

The probe phase is defined similarly:

$$
\begin{aligned}
\cos \left(\left(\omega_{c}+\Delta-\omega_{m}\right) t-\phi_{p r b}\right) & +\cos \left(\left(\omega_{c}+\Delta+\omega_{m}\right) t+\phi_{p r b}\right) \\
& =2 \cos \left(\left(\omega_{c}+\Delta\right) t\right) \cos \left(\omega_{m} t+\phi_{p r b}\right) .
\end{aligned}
$$

The quadrature phase measured by the probes is then oriented at an angle $\phi=\phi_{\text {prb }}-\phi_{0}$ relative to $\hat{X}_{1}$. The signal measured by the probes is proportional to

$$
\left\langle\hat{X}_{\phi}^{2}\right\rangle=\left\langle\hat{X}_{1}^{2}\right\rangle \cos ^{2} \phi+\left\langle\hat{X}_{2}^{2}\right\rangle \sin ^{2} \phi
$$

as shown in Fig. 4.10 .

The relative phase between the pumps and probes can be measured by comparing the phase of the probe tones' beat tone at $\omega_{m}$ with the phase of the pumps' beat tone at $\omega_{m}$. To measure the beat tones of the pumps and probes, we split off some of the source power and feed it into power diodes, then filter off any high-frequency oscillations (Fig. 4.11). The power diodes measure the beat power rather than amplitude, so they detect signals oscillating at $2 \times \omega_{m}$. The phase difference between the two diode outputs is thus twice $\phi$.

In order for the probe BAE tones to measure the full $2 \pi$ phase space of the squeezed state, we must halve the frequencies of the diode signals. To do this, we produce two reference signals at $\omega_{m}$ with an arbitrary function generator, double the frequency of the references, and lock the resulting $2 \omega_{m}$ tones to the diode signals. To lock the references to the diode signals, we measure the phase difference between them with a phase detector and use the resulting signal as an external modulation input to the function generator. When the phases are locked, the output of the phase detector is 0 , and the function generator stops modulating the reference. We then measure the phase difference between the two locked $\omega_{m}$ signals with a lock-in. 


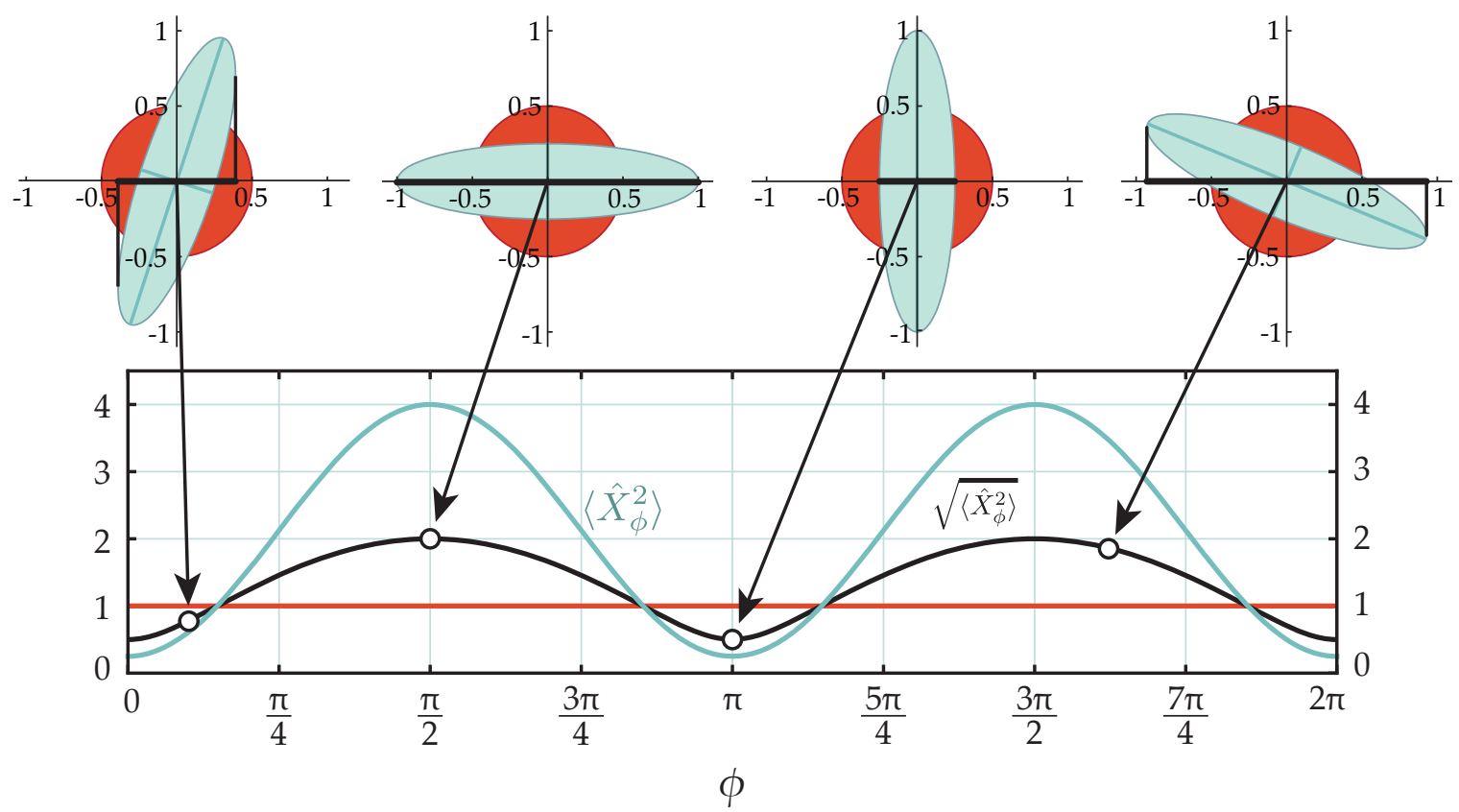

Figure 4.10: Top: Cartoon of a thermal state (red) and a squeezed state (turquoise) in phase space. Here, the $\mathrm{x}$-axis is the probes' measurement axis, and the squeezed axis is rotated by $\phi=\pi / 10, \pi / 2$, $\pi$, or $13 \pi / 8$, from left to right. The full width of the mechanical state in the $\hat{X}_{1}$ or $\hat{X}_{2}$ direction represents the root-mean-squared fluctuations in that quadrature, so $\sqrt{\left\langle\hat{X}_{1}^{2}\right\rangle}=\sqrt{\left\langle\hat{X}_{2}^{2}\right\rangle}=1$ for the thermal state, and $\sqrt{\left\langle\hat{X}_{1}^{2}\right\rangle}=1 / 2, \sqrt{\left\langle\hat{X}_{2}^{2}\right\rangle}=2$ for the squeezed state. The projection of the rms fluctuations onto the probe measurement axis is depicted as a heavy black line on the $\mathrm{x}$-axis. Bottom: The projection of the rms fluctuations on the probe axis vs. $\phi, \sqrt{\left\langle\hat{X}_{\phi}^{2}\right\rangle}$, is shown in black. The probes actually measure a signal proportional to $\left\langle\hat{X}_{\phi}^{2}\right\rangle=\left\langle\hat{X}_{1}^{2}\right\rangle \cos ^{2} \phi+\left\langle\hat{X}_{2}^{2}\right\rangle \sin ^{2} \phi$, shown in turquoise. At $\phi=0$ and $\pi$, the probes measure the fluctuations along $\hat{X}_{1}$, and at $\phi=\pi / 2$ and $3 \pi / 2$, they measure the fluctuations along $\hat{X}_{2}$. The thermal state's fluctuations are plotted in red. 


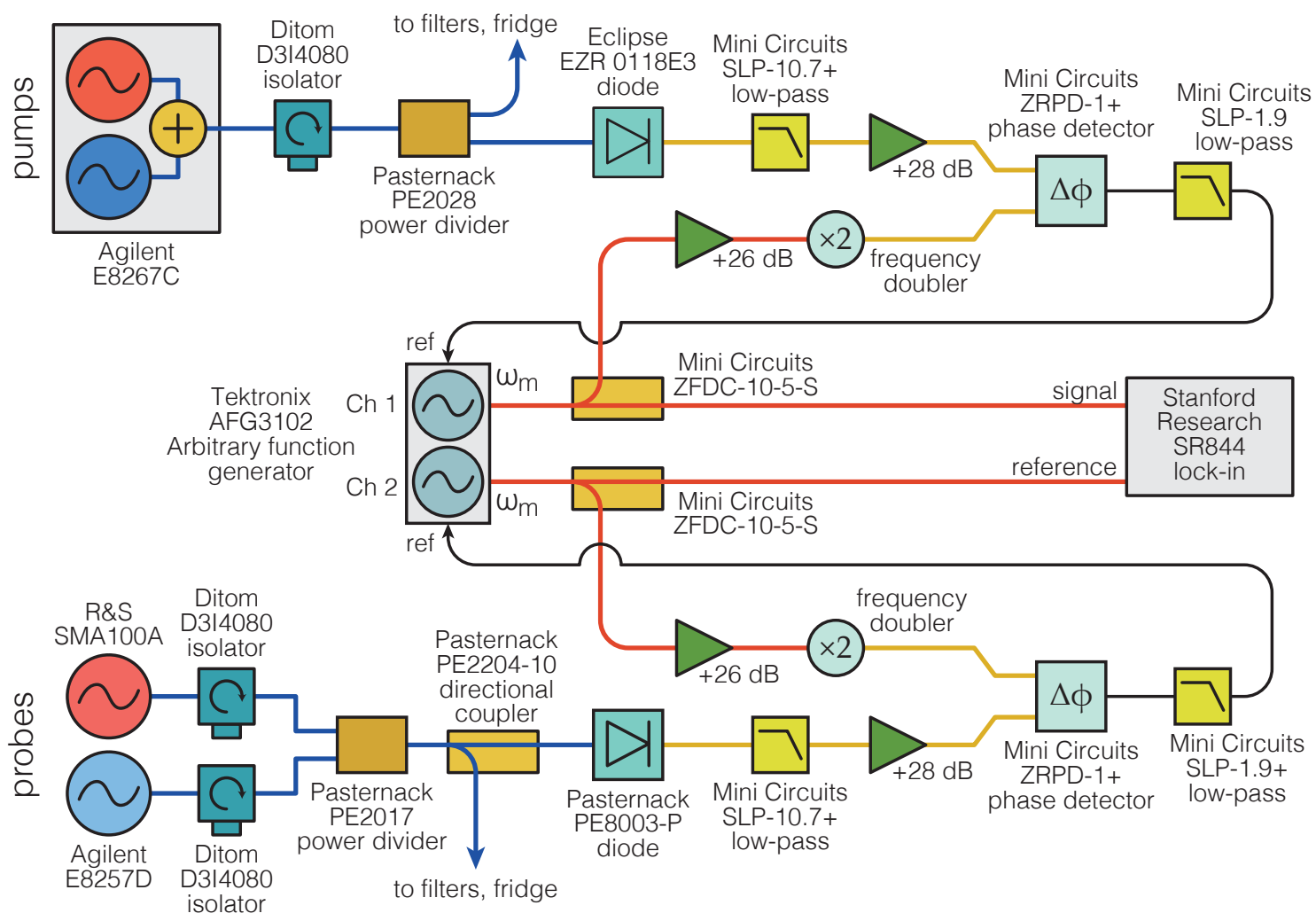

Figure 4.11: Circuit diagram for phase measurements. The two channels of an arbitrary function generator (middle) are locked to the beat frequencies of the pumps (top) and probes (bottom). The phase difference between the two channels is then measured with a lock-in (right). Blue lines indicate high-frequency signals, yellow lines indicate $2 \omega_{m}$ frequency signals, and red lines indicate $\omega_{m}$ frequency signals. 
We find that the phase measured in this manner is stable to within 1 degree over 5 minutes. We thus measure the phase every 5 minutes and adjust the probe phases to the desired value as a form of slow feedback. Measurements of the phase over the course of 1005 -minute segments give a standard deviation of $0.72^{\circ}$.

\subsubsection{Data processing}

To measure fluctuations on the zero-point level with our amplifier that adds more than 20 quanta of noise, we must average over many hours, or even days, to obtain acceptable levels of signal-to-noise. Even on these time scales, our system is very stable. We observe changes of less than $1 \%$ in the through power of our pump or probe tones, as shown in Fig. 4.12. The drift in through power we do see is most likely due to the frequency drift of our room-temperature filter cavities, as we know that their frequency is strongly temperature-dependent. Note that our measurement scheme should not be affected by system-wide changes in gain, as we always normalize the mechanical spectrum by the probe through power. Changing input gain can change the squeezing pump intra-cavity occupation, however, which could change the amount of squeezing in the mechanics. Frequencydependent changes in input gain, like those from shifting filter cavities, can also change the probe power ratio, which can introduce errors into the measured spectra. We thus reset the filter cavities and adjust pump powers twice a day to prevent large-scale drifts.

Over these long time scales, we do observe drifts of the system noise floor over time. These shifts are most likely due to the HEMT noise or the spectrum analyzer filter. Moreover, the noise floor is not flat, but instead has some curvature. We adjust for these drifts and take into account the curvature of the noise floor by switching between measurements of the probe sideband and the noise floor with the probes off (but with the squeezing tones still on). As we already must adjust the phase of the probes every $\sim 5$ minutes, as described in Section 4.3.1, we take this opportunity to switch between the sideband measurements and the floor measurements. Our complete measurement procedure is as follows:

1. Turn on pumps and probes. Adjust phase of probes.

2. Measure through powers of probes.

3. Measure probe sideband spectrum, averaging for $\sim 5$ minutes.

4. Turn off probes. Measure floor spectrum, averaging for $\sim 5$ minutes.

5. Repeat.

6. Every ten cycles, measure the pump through powers and the pump sideband spectrum for reference. 

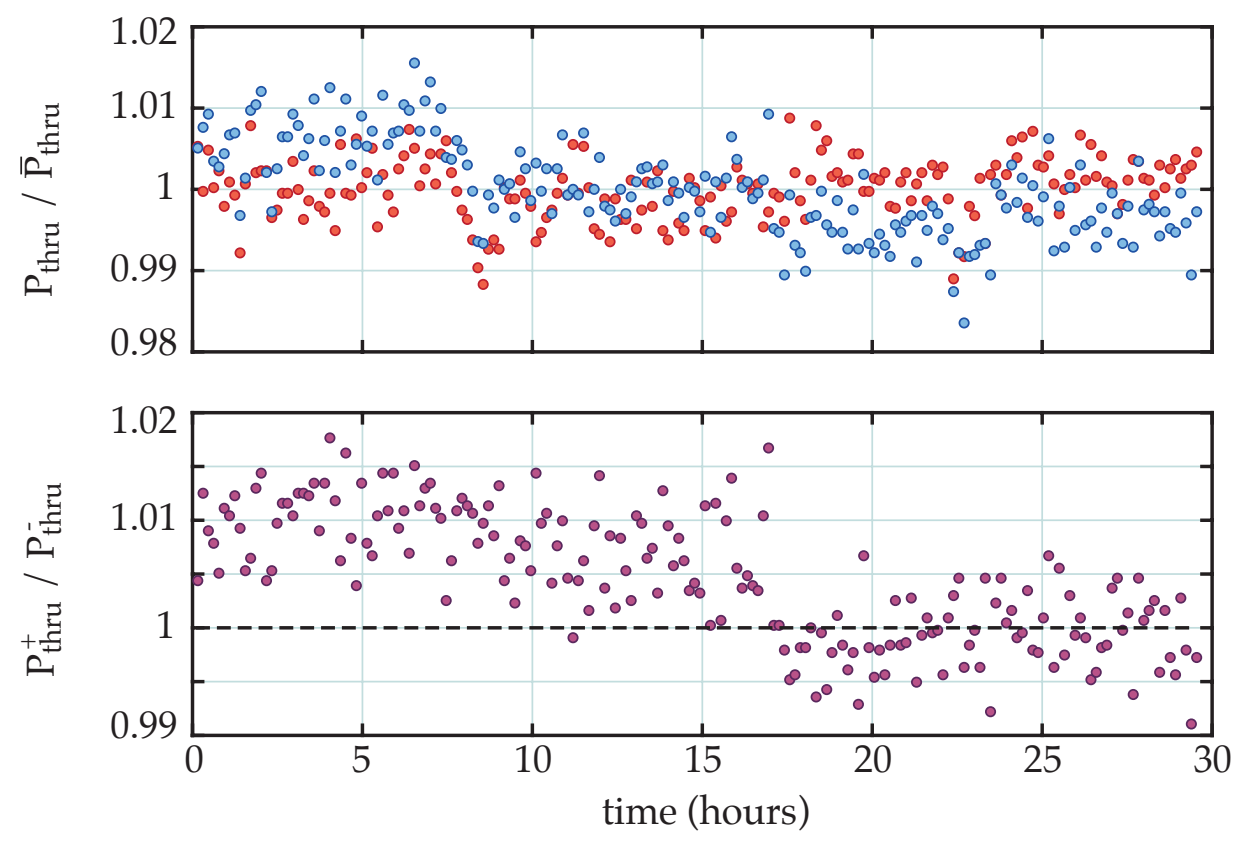

Figure 4.12: Gain stability vs. time. Top: Red and blue probe through power vs. time, normalized by the mean through value. The standard deviation is $0.38 \%$ for the red through and $0.57 \%$ for the blue through. Bottom: Probe through power ratio vs. time, normalized to the balancing ratio of $0.578 \pm 0.001$ obtained from the calibration described in Section 4.2.3. The mean ratio for this 30 hour period is 0.581 , with a standard deviation of 0.004 . 


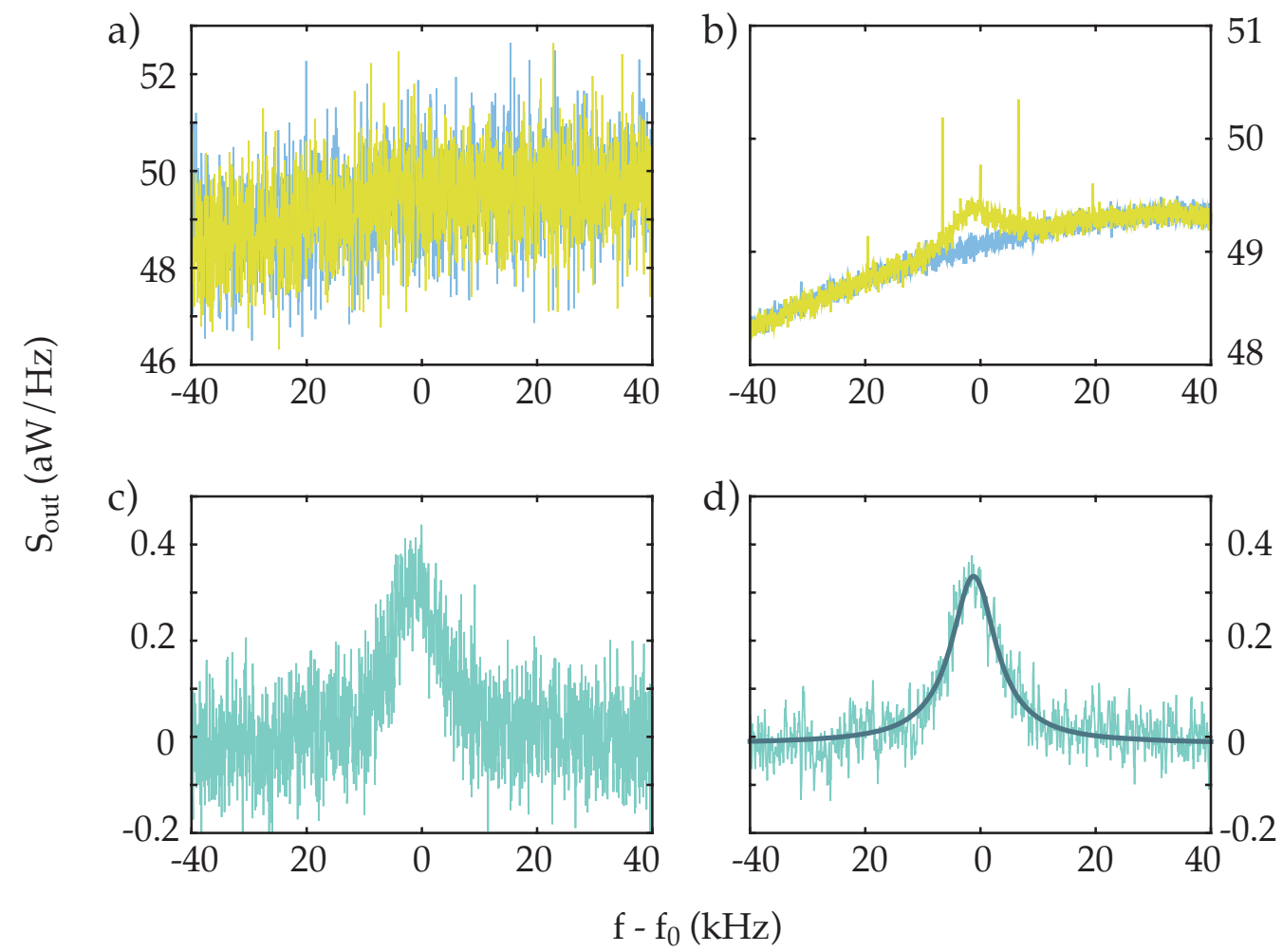

Figure 4.13: Processing and fitting the probe sideband. The spectra shown here are for the smallest noise signal we measured; less averaging is required for larger signals. $f_{0}$ is the sideband frequency at $\left(\omega_{c}+\Delta\right) / 2 \pi$. a) Spectra of the sideband (green) and floor (blue) with $\sim 5$ minutes of averaging for each. b) 261 5-minute spectra averaged together for the sideband (green) and floor (blue). The curved shape of the floor is apparent, as are a few small spurs in the sideband spectrum. c) Averaged sideband spectrum after subtracting the background and removing the spurs. d) Lorentzian fit to the spectrum shown in (c). The spectrum shown here has been smoothed by 5 bins to make it easier to compare to the fit curve. All other spectra shown in the figure are binned so that each bin width is twice the spectrum analyzer resolution bandwidth.

We then subtract the floor spectrum from the sideband spectrum before fitting the Lorentzian sideband. The subtraction and fitting procedure is illustrated in Fig. 4.13.

After averaging the 5-minute spectra, subtracting the noise floor, and removing any spurs, we bin the resulting spectrum by twice the spectral resolution bandwidth to ensure that neighboring bins are uncorrelated. We fit a Lorentzian to the final spectrum using MATLAB's "fit" function with the NonlinearLeastSquares option. Our free parameters are the Lorentzian area, linewidth, center frequency, and an overall linear offset. We can then convert the normalized sideband area to units of $x_{z p}^{2}$ using the thermal calibration from Section 4.2.1, Eq. 4.2. Note that, as the BAE sideband is proportional to twice the single-tone damping rate (Eq. 2.61), we divide the mechanical sideband area by twice the red-detuned through power in order to use the same calibration. The error derived in Section 2.7.2 for imbalances between the red- and blue-detuned probe tones assumes 


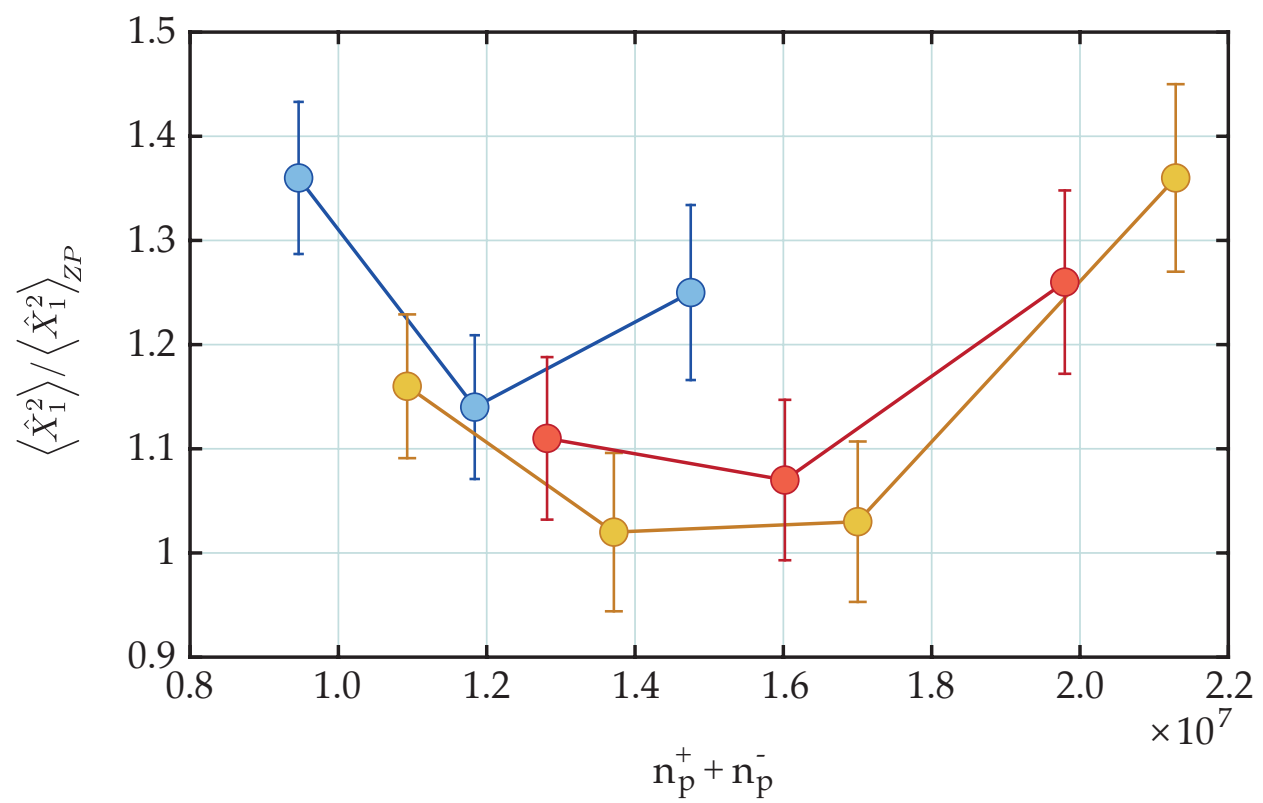

Figure 4.14: Parameter space search for optimum squeezing. Blue: $n_{p}^{+} / n_{p}^{-}=0.3$. Yellow: $n_{p}^{+} / n_{p}^{-}=$ 0.2 . Red: $n_{p}^{+} / n_{p}^{-}=0.1$. The 0.2 and 0.1 ratio data are not significantly different, so the larger ratio was chosen because sidebands with narrower linewidths are easier to measure.

that the calibration is done in this manner, as the sideband Lorentzian includes an overall factor of $\gamma_{\text {op }}^{-}$.

As we collect up to 261 of the 5-minute spectra, we have an independent way to check the error bars generated by the fitting routine. We can fit the individual spectra (or, more realistically, fit averages over several spectra) to gather statistics on the fit parameters produced. We find that the standard error of the fit parameters generated for different spectra matches the error bars produced by the fitting routine as long as the fitted spectrum is minimally-binned (i.e., so that there are the maximum number of uncorrelated bins in the spectrum). If the spectrum is not minimally-binned, the fit routine tends to overestimate the error bars. To calculate the final error bars of the quadrature fluctuations, we include the thermal calibration error and any error due to imbalance in the probes and the uncertainty of the balancing calibration. With balancing within $0.02 \mathrm{~dB}$, and estimating $n_{c}^{t h} \sim<1$ and $n_{c, R}^{t h}=0.37$, we can use Eq. 2.66 to find that the error due to any probe imbalance is $\leq 1 \%$.

\subsubsection{Parameter search}

As seen in Section 2.4.3, the $\hat{X}_{1}$ quadrature variance depends on both the total pump power and the $n_{p}^{+} / n_{p}^{-}$pump power ratio. We thus perform a rough search of this parameter space. Using the procedure described in the previous section, we take measurements for a range of phases at a given squeezing pump ratio to find the probe phase associated with $\hat{X}_{1}$, i.e., the phase with the 


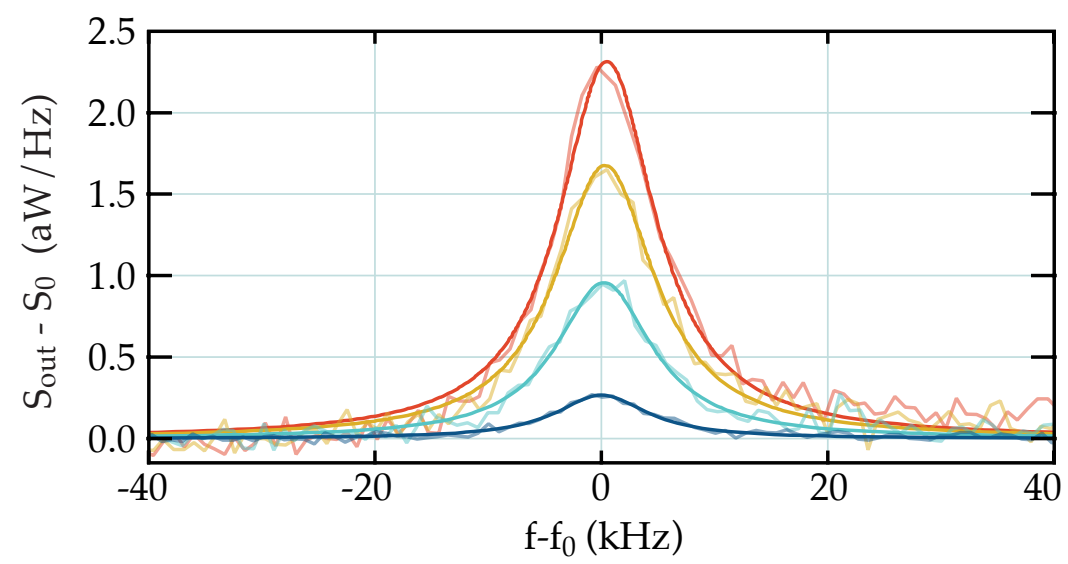

Figure 4.15: BAE probe spectra at $-81^{\circ},-51^{\circ},-21^{\circ}$, and $9^{\circ}$, from blue to red. Lorentzian fits obtained via the procedure described in Section 4.3.2 are shown in dark, solid lines. The spectra have been smoothed to make it easier to compare to the fit curves. Here, $f_{0}$ is the sideband centroid frequency. The spectrum at $-81^{\circ}$ is the same as that depicted in Fig. 4.13.

minimum probe sideband area. As this phase should not change as long as the pump ratio is fixed, we then change the total pump power and look for the smallest signal. We then change the ratio, find the minimum phase for that ratio, and change the total pump power again. Fig. 4.14 shows the obtained $\hat{X}_{1}$ variance vs. total pump power for several ratios. Using this rough search method, we find that optimum squeezing is produced when $n_{p}^{-}=1.131 \times 10^{7}$ and $n_{p}^{+}=0.232 \times 10^{7}$, with a ratio of $n_{p}^{+} / n_{p}^{-}=0.2$. We keep the total probe power a factor of 10 below the squeezing pump power, with each $n_{p}^{\text {probe }}=6.2 \times 10^{5}$. To check that the probes are not interfering with the state of the mechanics, we decrease the probe power by a few $\mathrm{dB}$ and find that the measured occupations are unchanged.

\subsubsection{Phase sweep}

Once the optimum pump and probe powers are found, we measure phases between $-170^{\circ}$ and $170^{\circ}$ in steps of $10^{\circ}$. Four of these spectra and fits are shown in Fig. 4.15.

As the BAE probes measure the projection of the fluctuations onto an axis oriented with angle $\phi$ with respect to the $\hat{X}_{1}$ axis (see Sec. 4.3.1 and Fig. 4.10), the sideband area should look like $\cos 2 \phi$ plus some offset. The probes measure the fluctuations in $\hat{X}_{1}$ at $0^{\circ}$ and $180^{\circ}$, and the fluctuations in $\hat{X}_{2}$ at $90^{\circ}$ and $270^{\circ}$. However, the probe phase measured by the lock-in at room temperature is not necessarily the same as $\phi$ in the cavity, so there will be some phase shift in the measured fluctuations from Eq. 4.6. This shift is evident in the measured quadrature variance vs. phase shown in Fig. 4.16.

From Fig. 4.16, $\hat{X}_{1}$ is squeezed to a level of $\left\langle\hat{X}_{1}^{2}\right\rangle=1.09 \pm 0.06 x_{z p}^{2}$, while $\hat{X}_{2}$ has a variation of $\left\langle\hat{X}_{2}^{2}\right\rangle=6.95 \pm 0.12 x_{z p}^{2}$. Using Eqs. 2.38 and 2.39, we can extract thermal bath occupations of $n_{c}^{\text {th }} \sim$ 0.75 and $n_{m}^{t h} \sim 70$. Referring back to Fig. 4.9, both bath occupations are lower than those estimated 


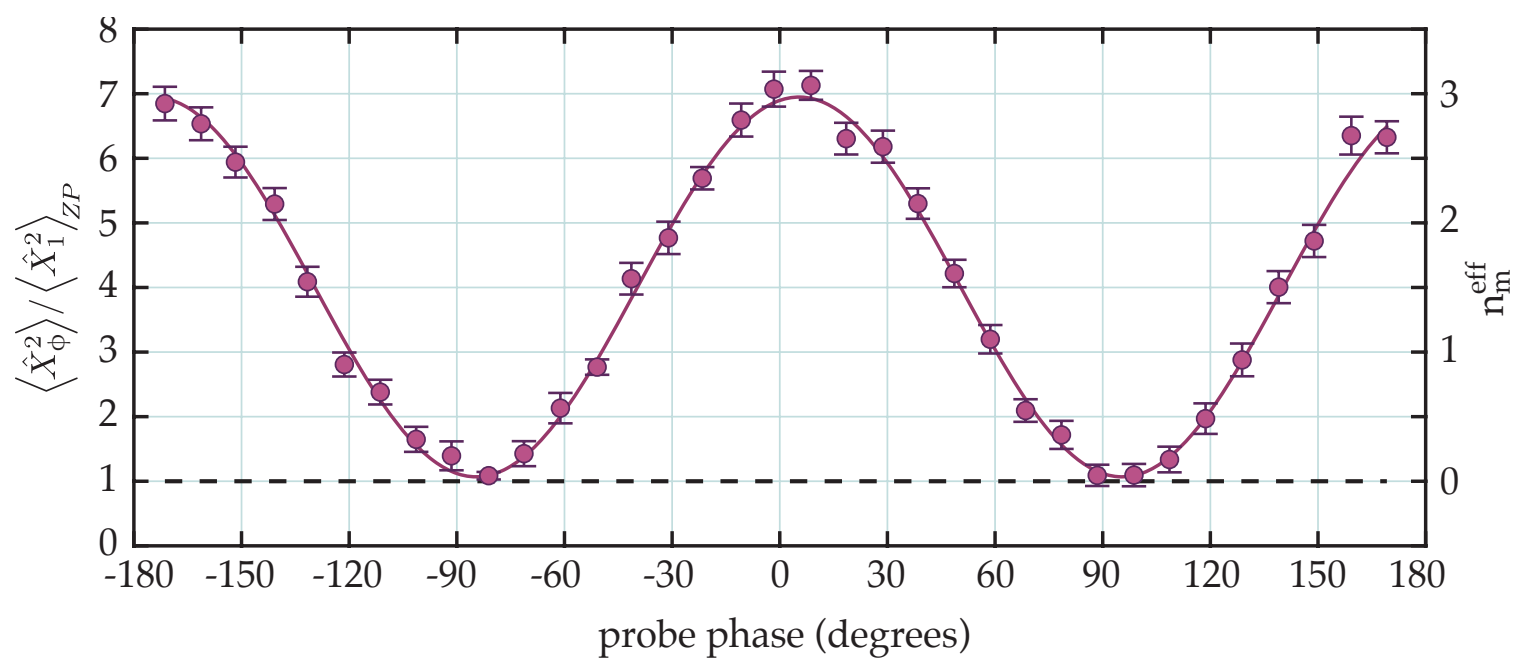

Figure 4.16: BAE probe measurement of $\left\langle\hat{X}_{\phi}^{2}\right\rangle$ vs. phase. The zero-point level is indicated by a dashed, black line. The minimum point at $-81^{\circ}$ has fluctuations at the level of $1.09 \pm 0.06 x_{z p}^{2}$. A sine fit to all the data (solid purple line) yields a minimum variation of $1.07 \pm 0.05 x_{z p}^{2}$. The effective quadrature occupation, defined as the occupation of a thermal state that would produce the given $\left\langle\hat{X}_{\phi}^{2}\right\rangle$ fluctuations, is plotted on the right axis. The point at $-81^{\circ}$ has an effective occupation of 0.043 quanta.

for the total pump power of $1.42 \mathrm{e} 7$ photons. However, there are significant differences between the conditions during the squeezing experiment and those for the cavity occupation estimation. The cavity occupation was extracted from measurements during a BAE experiment, where the total power is composed of two strong pumps of equal amplitude, whereas the blue-detuned squeezing pump has 0.2 times the power of the red-detuned pump here. As we will see in Appendix B, applying two tones of equal power can result in as much as a factor of 5 increase in the cavity thermal occupation compared to when the same total power is applied with a single, red-detuned pump. Moreover, the cooling experiment and $n_{c}^{\text {th }}$ measurements were both done at $20 \mathrm{mK}$, whereas the squeezing experiments took place at $10 \mathrm{mK}$.

Another sign that the cavity and mechanical bath heating may be dependent on the pump ratio is that the optimum pump ratio we found in the parameter space search (Section 4.3.3) is lower than we'd expect. If we assume that the heating is fixed at $n_{c}^{t h}=0.75$ and $n_{m}^{t h}=70$ for a total pump power of $n_{p}^{-}+n_{p}^{+}=1.363 \times 10^{7}$, the optimum pump ratio should be closer to $n_{p}^{+} / n_{p}^{-}=0.7$ rather than 0.2. As seen in Fig. 4.14, the $\hat{X}_{1}$ variance at a ratio of 0.3 (blue) is alread higher than the variance at a ratio of 0.2 (yellow) at a total pump occupation of $1.363 \times 10^{7}$.

At a temperature of $10 \mathrm{mK}$, the $4.0 \mathrm{MHz}$ mechanical mode should start out with fluctuations of $\left\langle\hat{X}_{1}^{2}\right\rangle=\left\langle\hat{X}_{2}^{2}\right\rangle=105\left\langle\hat{X}_{1}^{2}\right\rangle_{Z P}$, so both quadratures are damped. This makes it difficult to define the amount of squeezing. If we consider only the net damping from the excess red pump power acting on the mechanics, and take the bath occupations to be $n_{c}^{t h} \sim 0.75$ and $n_{m}^{t h} \sim 70$ as extracted above, 
we'd expect that the mechanics would be cooled to $\bar{n}_{m}=0.82$, with fluctuations of $\left\langle\hat{x}^{2}\right\rangle=2.6 x_{z p}^{2}$. Our measured $\hat{X}_{1}$ variance has about $3.9 \mathrm{~dB}$ of squeezing compared this value.

\subsection{Discussion}

These measurements represent the first implementation of a reservoir engineering approach to squeeze the motion of a mechanical resonator. They are also the first continuous BAE measurements of a mechanical squeezed state. While we were not able to produce a quantum squeezed state, we demonstrated more than $3 \mathrm{~dB}$ of squeezing, stable on timescales of days to weeks.

It is unfortunate that we did not have a better way to measure the cavity and mechanical thermal bath occupations at the time of these measurements, as it would have been useful to compare the expected squeezing from Eq. 2.38 to the measured squeezing with the BAE probes. These techniques were developed later by forming more accurate models of our system beyond the weak-coupling or single-tone limits, as described in Chapter 2. By the time we cooled down the device described in Chapter 5, we had already implemented full spectral response and driven response models on several devices, and found them to be a useful tool for extracting the parameters of our system. 


\section{Chapter 5}

\section{Device 2: Full-spectrum measurements}

\subsection{Overview}

As the previous device failed to squeeze below the zero-point fluctuations due to the presence of power-dependent heating, we sought to minimize the required power by fabricating a new device with higher coupling. We tested several alternative devices, but results presented here are from the device with the most extensive testing and measurements (D2). The relevant parameters for D2 are shown in Table 5.1. Note that $g_{0}$ for this device is a factor of two larger than $g_{0}$ for D1.

For this device, $\kappa$ is almost half that of D1. This is advantageous for squeezing, since we are able to achieve high cooperativities at lower pump powers. However, at the optimum squeezing pump powers for this device, the mechanical linewidth is broadened to more than $\kappa / 10$. To use the BAE probe measurement scheme from the last chapter for this device, it would thus be necessary to place the probe sideband outside the bandwidth of the cavity, decreasing measurement sensitivity and increasing measurement time. As the previous measurements already took weeks to complete, this is not a feasible measurement scheme for this device.

Instead, we used a full model of the squeezed driven and spectral responses to extract the occupations of the thermal baths. With our calibrations and the results of these fits, we know all parameters of the Hamiltonian discussed in Chapter 2 and can thus extract the quadrature

\begin{tabular}{cc} 
Parameter & Value \\
\hline$\omega_{m}$ & $2 \pi \times 3.6 \mathrm{MHz}$ \\
$\gamma_{m}$ & $2 \pi \times 3 \mathrm{~Hz}$ \\
$\omega_{c}$ & $2 \pi \times 6.23 \mathrm{GHz}$ \\
$\kappa$ & $2 \pi \times 440 \mathrm{kHz}$ \\
$g_{0}$ & $2 \pi \times 35.6 \mathrm{~Hz}$ \\
$x_{z p}$ & $2.3 \mathrm{fm}$ \\
\hline
\end{tabular}

Table 5.1: Parameters for Device 2. 


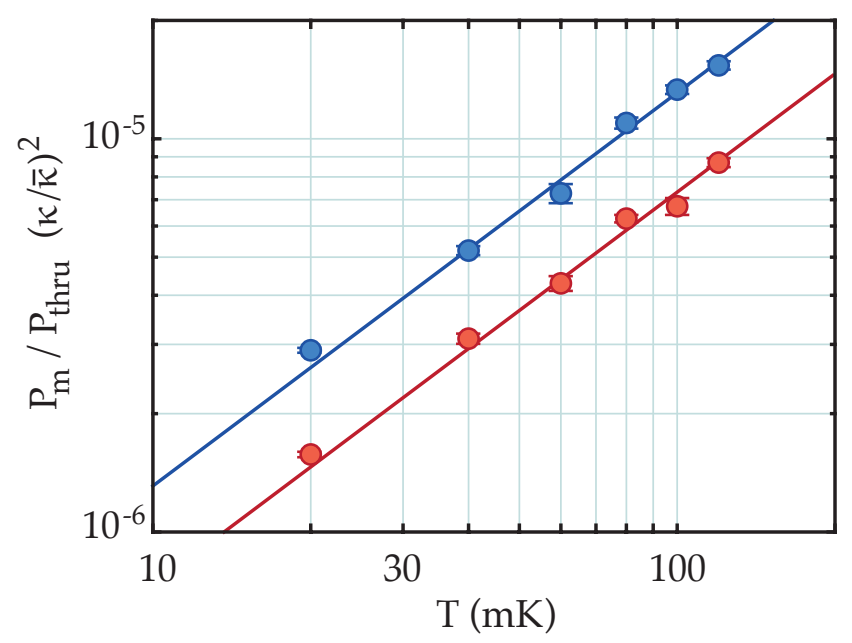

Figure 5.1: Normalized sideband power versus temperature for both red- and blue-detuned pumps.

variances. A similar model, in the limit of no blue-detuned pump power, has proved useful in cooling experiments where the broadened mechanical linewidth is comparable to $\kappa$ [56].

\subsection{Device calibrations and characterization}

\subsubsection{Thermal calibration}

As described in Section 3.4.2, we measure the integrated sideband powers of weak blue-detuned and red-detuned pumps versus temperature in order to calibrate the sideband area to known thermal occupations and to find $g_{0}$. To prevent the pumps from heating or cooling the mechanical state, we choose $n_{p}^{-}=n_{p}^{+} \sim 50$. As will be seen in Section 5.2.2, this corresponds to $\gamma_{o p}^{ \pm} \sim 2 \pi \times 0.5 \mathrm{~Hz}$ for each pump. For the temperatures in this calibration, $\gamma_{m} \geq 2 \pi \times 5 \mathrm{~Hz}$, and so balancing within $10 \%$ will damp or amplify the mechanics by less than $1 \%$.

As the cavity linewidth changes slightly with temperature, instead of fitting $P_{m} / P_{t h r u}$ vs. temperature as we did with the last device, we fit $P_{m} / P_{t h r u} \times(\kappa / \bar{\kappa})^{2}$ vs. temperature, where $\bar{\kappa}$ is the average linewidth for all temperatures. This ensures that the slope is a constant with respect to temperature:

$$
\left(\frac{P_{m}}{P_{\text {thru }}}\right)_{ \pm}\left(\frac{\kappa}{\bar{\kappa}}\right)^{2}=\frac{\omega_{c}}{\omega_{ \pm} \lambda_{ \pm}}\left(\frac{2 g_{0}}{\bar{\kappa}}\right)^{2} \frac{k_{B}}{\hbar \omega_{m}} T .
$$




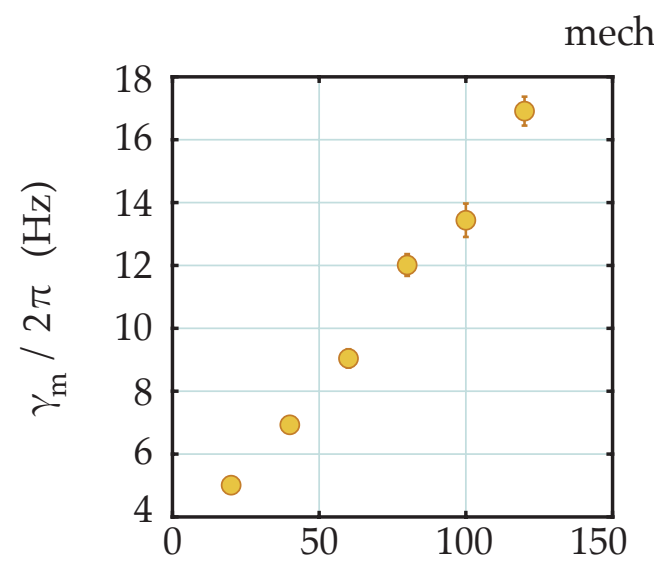

mechanics
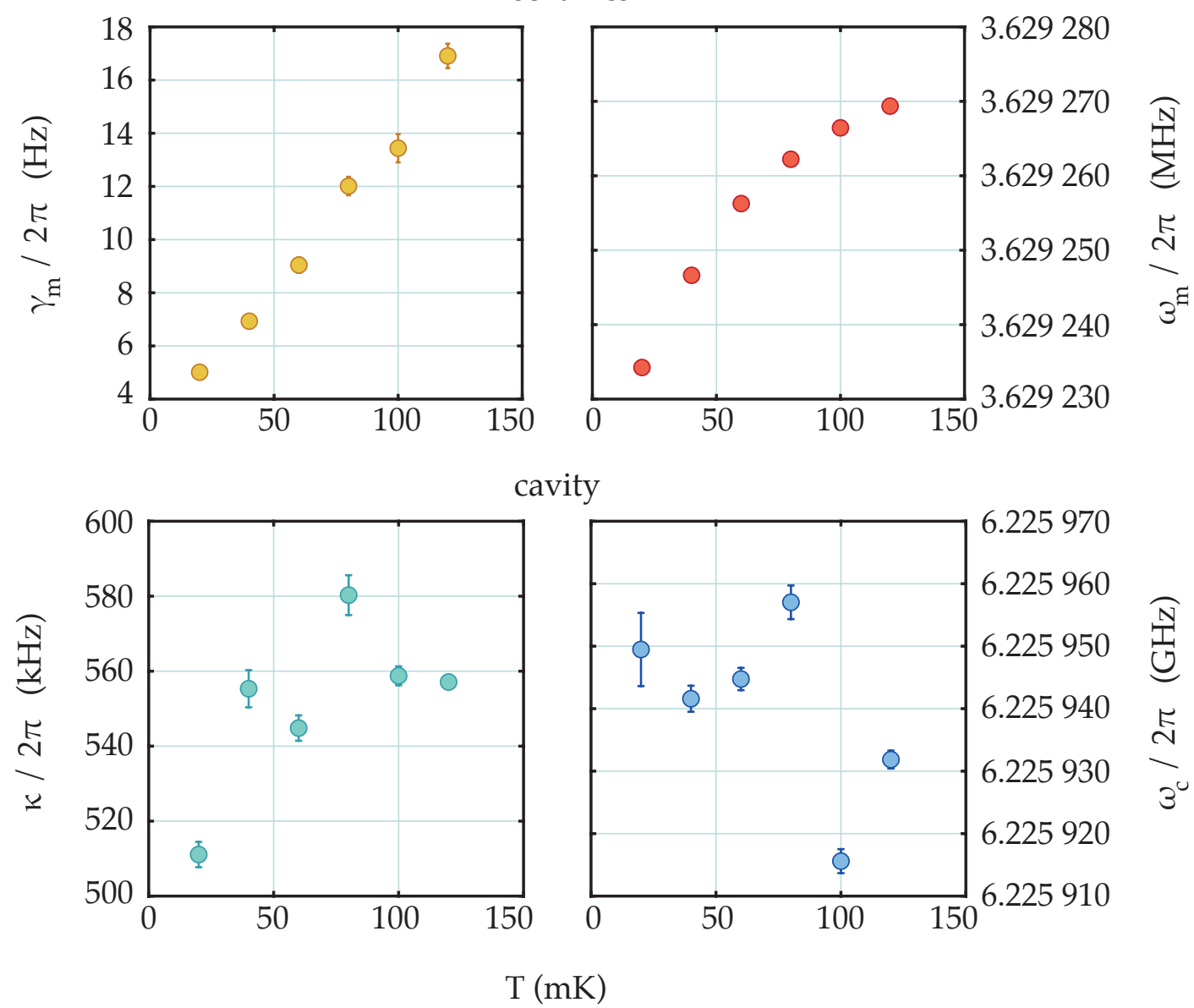

Figure 5.2: Mechanical and cavity frequencies and linewidths as a function of temperature.

We find

$$
\begin{gathered}
\left(\frac{P_{m}}{P_{\text {thru }}}\right)_{-}\left(\frac{\kappa}{\bar{\kappa}}\right)^{2}=(7.317 \pm 0.270) \times 10^{-5} \times T \\
\left(\frac{P_{m}}{P_{\text {thru }}}\right)_{+}\left(\frac{\kappa}{\bar{\kappa}}\right)^{2}=(13.097 \pm 0.418) \times 10^{-5} \times T .
\end{gathered}
$$

These fits are shown in Fig. 5.1. From these slopes, we find that the parasitic bypass channel parameter is $\xi=(0.145 \pm 0.012) \omega_{m}^{-1}$, and the optomechanical coupling rate is $g_{0}=2 \pi \times(35.61 \pm 0.45$ $\mathrm{Hz})$.

Fig. 5.2 shows the temperature-dependence of the device parameters. The mechanical linewidth and frequency both increase with temperature. The cavity linewidth shows a slight increase with temperature and the cavity frequency shows a slight decrease with temperature, but as there are 100 pump photons in the cavity, these parameters may be dominated by the presence of the pumps rather than the temperature of the cavity. 
a)

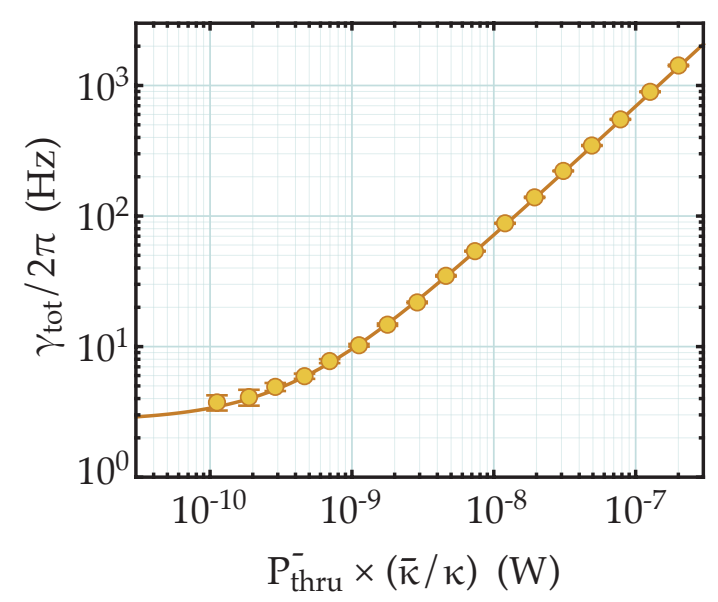

b)

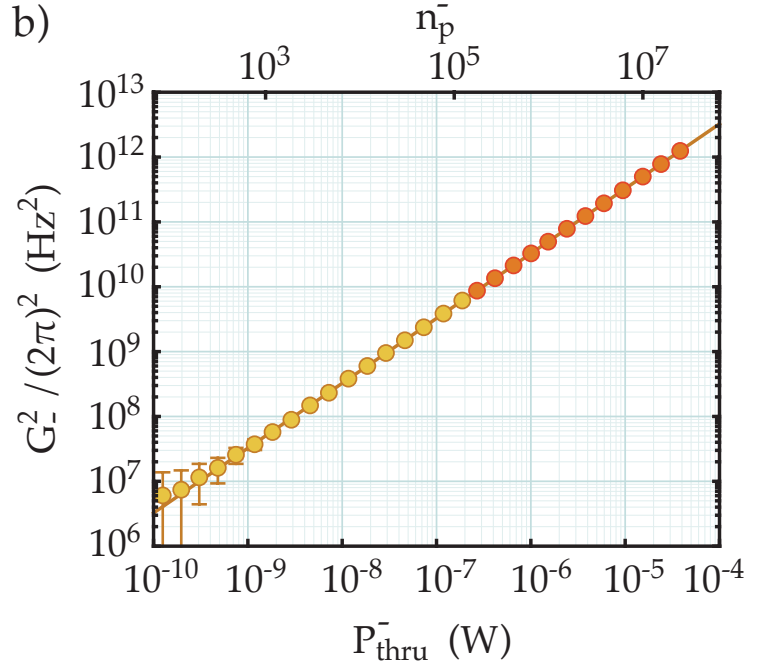

Figure 5.3: a) Linewidth broadening calibration. Yellow circles represent the total mechanical linewidth obtained by fitting a Lorentzian model to the mechanical driven response at different red-detuned pump powers. The solid yellow line represents a fit to the first 12 data points. The fit equation is given in Eq. 5.2. b) $G_{-}^{2}$ versus red-detuned pump power in the weak-driving regime (yellow circles) and the strong-coupling regime (red circles). Yellow circles represent values of $G_{-}^{2}$ obtained from Lorentzian fits using $\gamma_{t o t}=\gamma_{m}+4 G_{-}^{2} / \kappa$, and red circles represent values of $G_{-}^{2}$ obtained by fitting Eq. 2.74 to the driven response.

\subsubsection{Linewidth broadening}

To calibrate $G_{-}$to the measured pump through power, $P_{t h r u}^{-}$, we measure the mechanical linewidth vs. pump power via driven response, as described in Sec. 3.4.3. We observe considerable narrowing of the cavity linewidth with increasing pump power in this device, suggesting the presence of twolevel systems [18]. Instead of fitting $\gamma_{t o t}$ vs. $P_{t h r u}^{-}$, we fit $\gamma_{t o t}$ vs. $P_{t h r u}^{-} \times \bar{\kappa} / \kappa$. The resulting offset is then $\gamma_{m}$, and the obtained slope is $4 \beta_{-} / \bar{\kappa}$. We can thus obtain a calibration for $G_{-}^{2}=\beta_{-} \times P_{t h r u}^{-}$ for all measured through powers.

Fig. 5.3a shows the resulting mechanical linewidth vs. scaled through power. We apply a linear fit to the first 12 data points to ensure that $\gamma_{t o t} \ll \kappa$, and we obtain

$$
\begin{aligned}
\gamma_{t o t} / 2 \pi & =(2.563 \pm 1.405) \mathrm{Hz}+(7.042 \pm 0.197) \times 10^{9}(\mathrm{~Hz} / \mathrm{W}) \times P_{t h r u}^{-}(\bar{\kappa} / \kappa) \\
G_{-}^{2} & =(3.251 \pm 0.091) \times 10^{15} \frac{(\mathrm{rad} / \mathrm{s})^{2}}{\mathrm{~W}} \times P_{t h r u}^{-} .
\end{aligned}
$$

As we increase the pump power, the mechanical linewidth becomes comparable to the cavity linewidth, and the mechanical response can no longer be fit in the Lorentzian form of Eq. 2.75. In this regime, a full model of the driven response (Eq. 2.74) is necessary. When we extract $G_{-}^{2}$ from full fits to the driven response, we find that $G_{-}^{2}$ vs. $P_{t h r u}^{-}$is linear up to pump occupations greater than $n_{p}^{-}=10^{7}$ (Fig. 5.3b). As $G_{-}^{2}$ is proportional to $g_{0}^{2}$, this suggests that we have made a valid 

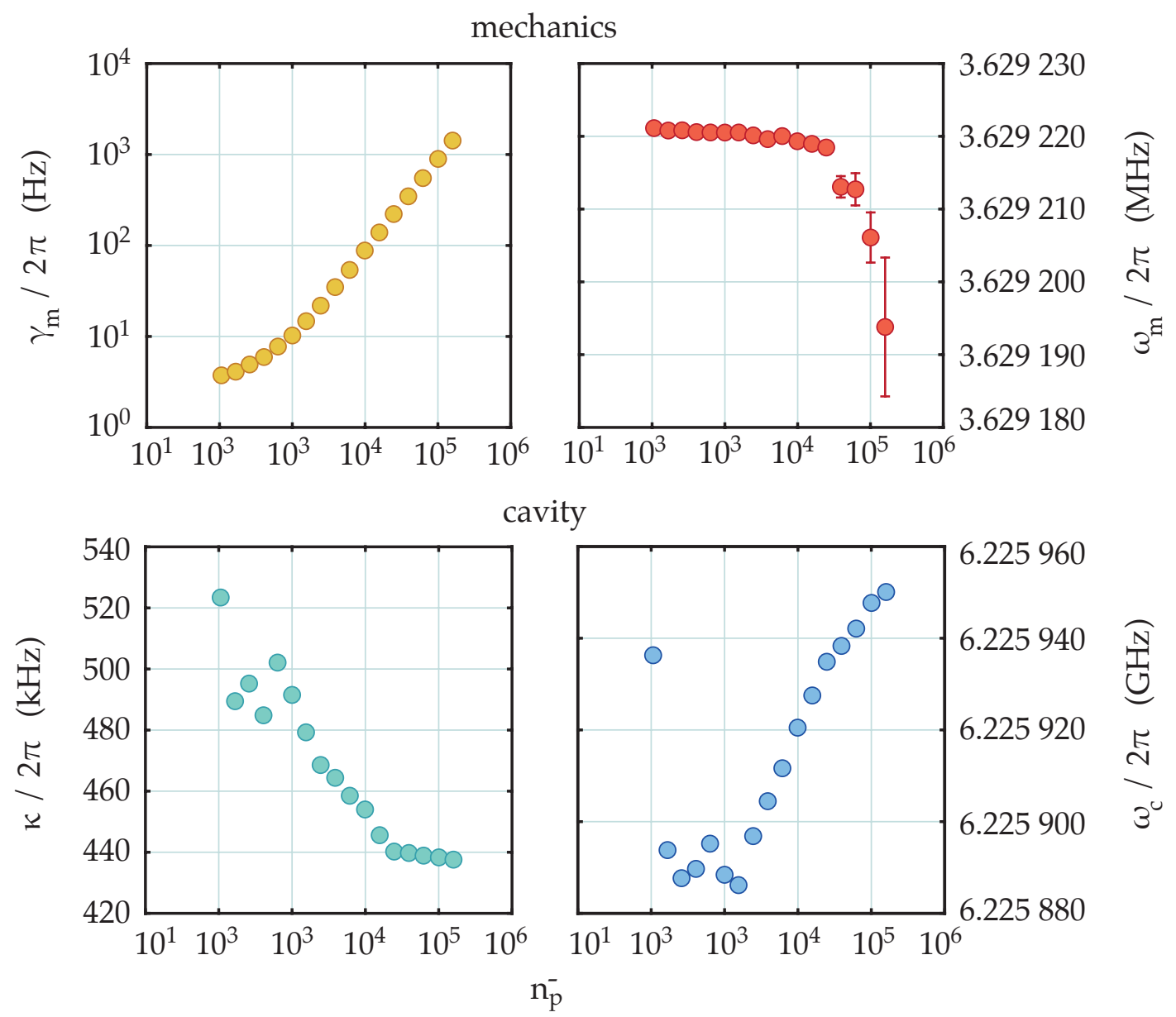

Figure 5.4: Mechanical and cavity linewidths and frequencies vs. pump power, determined through Lorentzian fits to both mechanical and cavity driven responses.

assumption that the single-photon optomechanical coupling rate is independent of pump power. It also shows that the full model derived in Chapter 2 accurately captures the behavior of the device when $\gamma_{t o t}$ is comparable to $\kappa$.

Fig. 5.4 shows the effects of increasing red-detuned pump power on the mechanical and cavity linewidths and frequencies. The mechanical frequency begins to decrease with increasing pump power. The cavity linewidth narrows, while the cavity frequency increases. This behavior is consistent with the presence of two-level systems (TLS) [52].

From the bypass channel parameter found in Section 5.2.1, we can also calculate a calibration for $G_{+}^{2}$ vs. $P_{t h r u}^{+}$:

$$
G_{+}^{2}=(5.817 \pm 0.290) \times 10^{15} \frac{(\mathrm{rad} / \mathrm{s})^{2}}{\mathrm{~W}} \times P_{t h r u}^{+}
$$

Combining the $G_{ \pm}^{2}$ calibrations with the value of $g_{0}$ obtained in Section 5.2.1, we can find calibrations 
for the intracavity pump occupations:

$$
\begin{aligned}
& n_{p}^{-}=(6.492 \pm 0.244) \times 10^{11} \frac{1}{\mathrm{~W}} \times P_{t h r u}^{-} \\
& n_{p}^{+}=(11.621 \pm 0.716) \times 10^{15} \frac{1}{\mathrm{~W}} \times P_{t h r u}^{+}
\end{aligned}
$$

\subsubsection{Noise floor}

In order to deal with noise floors that are not perfectly flat over the linewidth of the cavity, we take spectra with the pumps turned off and subtract these noise floors from the spectra measured with the pumps on. We used a similar procedure for our BAE probe measurements (Sec. 4.3.2), although the noise floor was taken with the squeezing pumps on. We used the no-pump-floor method with several other devices, and it produced spectra with flat noise floors outside of the cavity bandwidth (see, for example, the green spectrum in Fig. 5.9).

With this device, however, the noise floor appears to have a small peak on top of a linear background, as shown in Fig. 5.5a. Just as the observation of a dip in the noise floor in the absence of pumps is a sign of cavity occupation from the right port (Section 4.2.4), a peak in the no-pump floor could indicate a non-zero $n_{c, i n t}^{t h}$ or $n_{c, L}^{t h}$. If we subtract the linear background, we can fit a Lorentzian to the noise floor. The fit gives a linewidth of $990 \mathrm{kHz}$ and an area corresponding to an occupation of 0.22 quanta.

There are several reasons why it is unlikely that this peak in the noise floor represents a physical cavity occupation as opposed to being an artifact of a slight frequency-dependence in the HEMT noise or output line gain. First of all, at the fridge base temperature, the linewidth of this device is always measured to be $\leq 550 \mathrm{kHz}$, even in the presence of drive powers of only a few photons. It is thus unlikely that the cavity would have a linewidth of almost $1 \mathrm{MHz}$ when occupied by 0.22 photons. Secondly, it is unlikely that the occupation is due to $n_{c, \text { int }}^{t h}$, as the device is well-thermalized to the mixing stage, as seen from measurements of the mechanical occupation. It is theoretically possible for noise on the input line to be less attenuated than we would expect, creating an $n_{c, L}^{t h}$ larger than the expected occupation of 0.025 quanta (Section 3.3.1). However, as $n_{c}^{t h}=\Sigma_{i} \frac{\kappa_{i}}{\kappa} n_{c, i}^{t h}$, where $\frac{\kappa_{L}}{\kappa} n_{c, i}^{t h}<0.2$, if the 0.22 photons in $n_{c}^{t h}$ are due only to $n_{c, L}^{t h}$, then $n_{c, L}^{t h}=\frac{\kappa}{\kappa_{L}} n_{c}^{t h}=1.1$, which corresponds to a physical temperature of over $450 \mathrm{mK}$. It is unlikely that our input line, with its 20 $\mathrm{dB}$ of attenuation at both $4 \mathrm{~K}$ and the mixing stage, would have such a high temperature, particularly since we do not observe any peaks in the noise floors of other devices at different frequencies. Most convincingly, when we measure the noise floor for the same frequency span at room temperature and perform the same analysis to subtract out the linear background, we see a peak of exactly the same scale as the cold no-pump floor (Fig. 5.5b).

It thus seems most likely that the peak or kink in the noise floor is merely an artifact of our 
a)

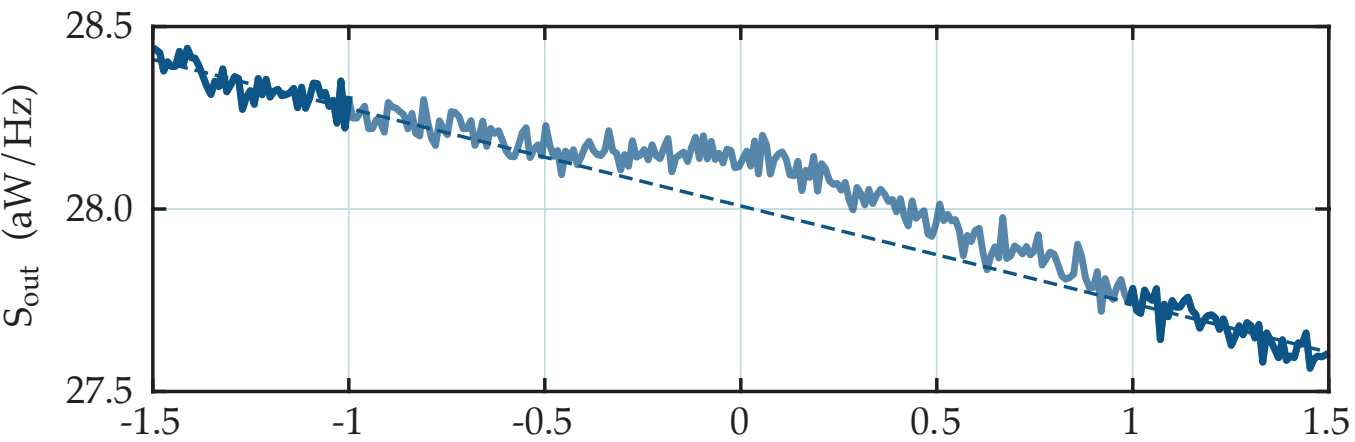

b)

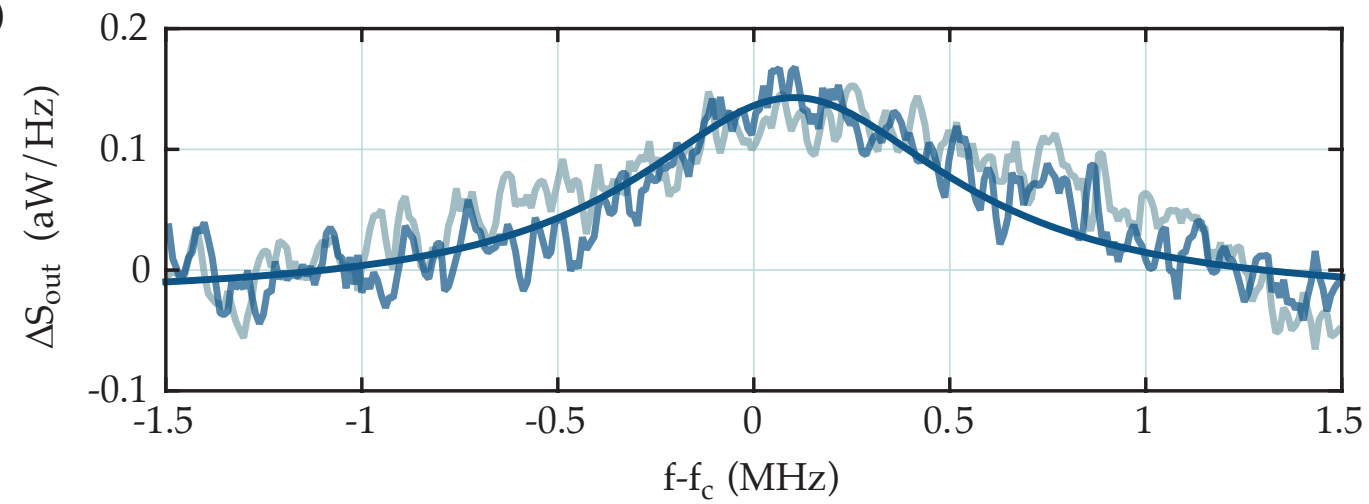

Figure 5.5: Noise floor analysis. a) Averaged noise floor spectrum, showing an apparent peak on top of a linear background. The dashed line represents a linear fit to the sections of the spectrum shown in dark blue. b) Transparent blue: Spectrum from (a) with the linear background subtracted. Solid blue: Lorentzian fit to the background-subtracted spectrum. The Lorentzian linewidth is 990 $\mathrm{kHz}$, and the area gives $n_{c}^{t h}=0.22$. Pale gray: room temperature noise floor with the same linear background subtraction procedure applied. 
measurement system noise. Moreover, it is worth noting that this is a small effect. If we do not subtract off the no-pump noise floors and instead modify the data analysis procedure described in Section 5.3.1 to fit the floor with a linear background, our optimal squeezing point changes by $10 \%$, or about two error bars.

\subsubsection{Cooling measurements}

To estimate the bath heating vs. power for this device, we first perform a cooling measurement. We apply a single, red-detuned pump and measure the output spectrum. At lower pump powers, the mechanical linewidth is much smaller than the cavity linewidth. We thus fit the mechanical sideband with a Lorentzian to find the sideband area, which is proportional to $\bar{n}_{m}-2 n_{c}^{t h}$ when $n_{c, R}^{t h}=0$. We then fit the cavity spectrum with a Lorentzian to find $n_{c}^{\text {th }}$ and extract the mechanical occupation.

At higher pump powers, the mechanical linewidth becomes comparable to the cavity linewidth, and a Lorentzian model of the sideband can no longer describe the measured spectra. We thus fit the full model from Eq. 2.77. To determine $\kappa$ and $G_{-}$, we first fit the driven response to a full model from Eq. 2.74. Our remaining free parameters are then $n_{m}^{t h}, n_{c}^{t h}, \omega_{m}, \omega_{c}$, and a constant offset. The mechanical linewidth is determined from a linear fit to $\gamma_{m}$ vs. $n_{m}^{t h}$, obtained from thermal calibration data (see the upper left panel of Fig. 5.2), but fits that assume that $\gamma_{m}=0$ yield identical results, as $\gamma_{m}<<\gamma_{t o t}$ in the strong-coupling regime. $\bar{n}_{m}$ is then calculated by plugging the measured frequencies and bath occupations into Eq. 2.71. The results of the cooling measurements are shown in Fig. 5.6.

At a pump power of $8.6 \mathrm{e} 5$, we obtain a minimum occupation of $\bar{n}_{m}=0.22 \pm 0.08$. This is the coldest mechanical occupation that we have measured in the group to this date, and is comparable to the coldest steady-state occupations produced in other groups [56]. Using the extracted bath occupations vs. applied power, we can also estimate the squeezing we'd expect for our device. Assuming that the heating for a total squeezing pump power $n_{p}^{-}+n_{p}^{+}$is the same as the measured heating for a single red-detuned tone with the same total power, we can calculate $\left\langle\hat{X}_{1}^{2}\right\rangle$ vs. pump ratio from Eq. 2.38, and thus find the ratio that produces the minimum value of $\left\langle\hat{X}_{1}^{2}\right\rangle$. The results of these calculations are shown in Fig. 5.7. Squeezing appears to be possible for total pump occupations above $10^{7}$.

\subsubsection{Excess amplifier phase noise}

When we first performed measurements of cooling for this device, we observed an increasing slope in the noise floor as we turned up the red-detuned pump power, as seen in Fig. 5.8. This suggests that there is some power-dependent noise source in our system. It is important to figure out where the noise is arising: if it is a source that occurs in the circuit before the device input, then the 


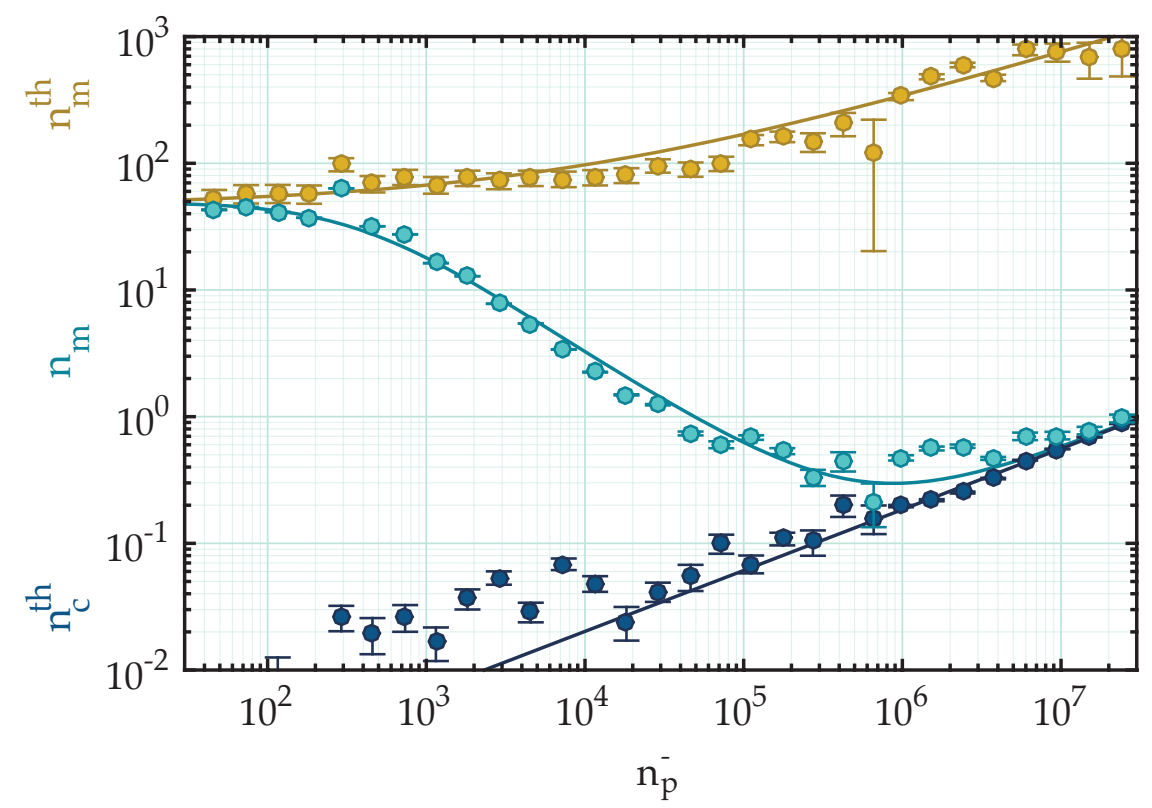

Figure 5.6: Sideband cooling measurements for increasing red-detuned pump power. Low-power spectra are fitted using Lorentzian models for both the cavity and mechanical spectra, while highpower spectra are fitted with a full model. Solid lines represent power-law fits to $n_{m}^{t h}$ and $n_{c}^{t h}$.
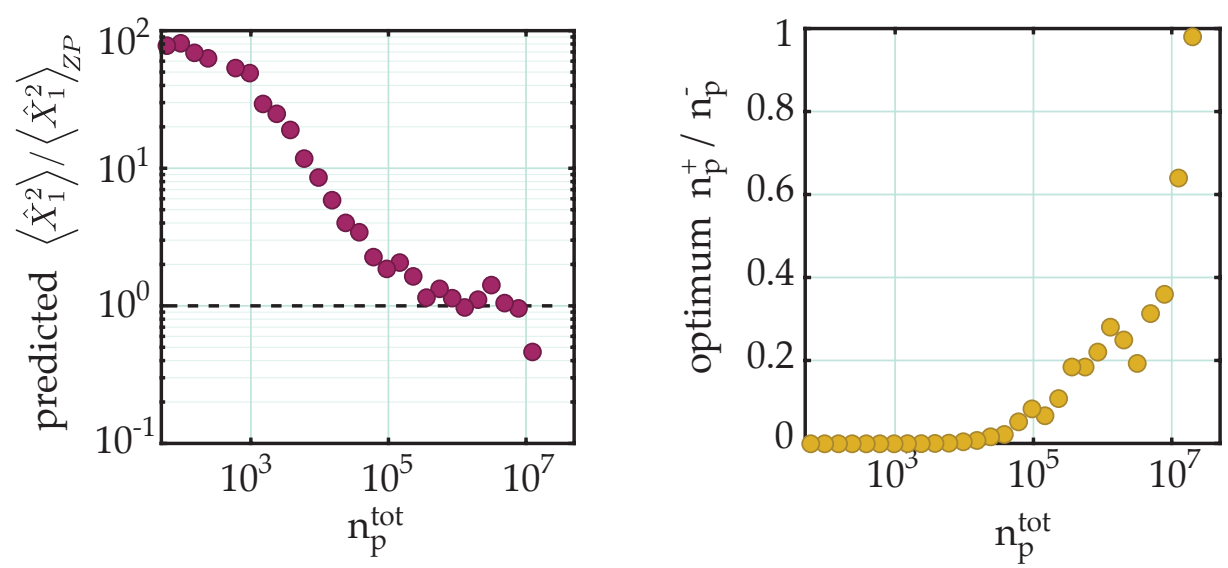

Figure 5.7: Based on the bath occupations vs. red-detuned power (Fig. 5.6), we estimate the amount of squeezing possible if the bath heating at a total squeezing pump power, $n_{p}^{-}+n_{p}^{+}$, is the same as the heating at the corresponding red-detuned power during cooling. Left: predicted squeezing at the optimum pump ratio for the given total power. Right: optimum pump ratio. Squeezing appears to be possible above $n_{p}^{-}+n_{p}^{+}=10^{7}$, with optimum ratios above 0.5 . 


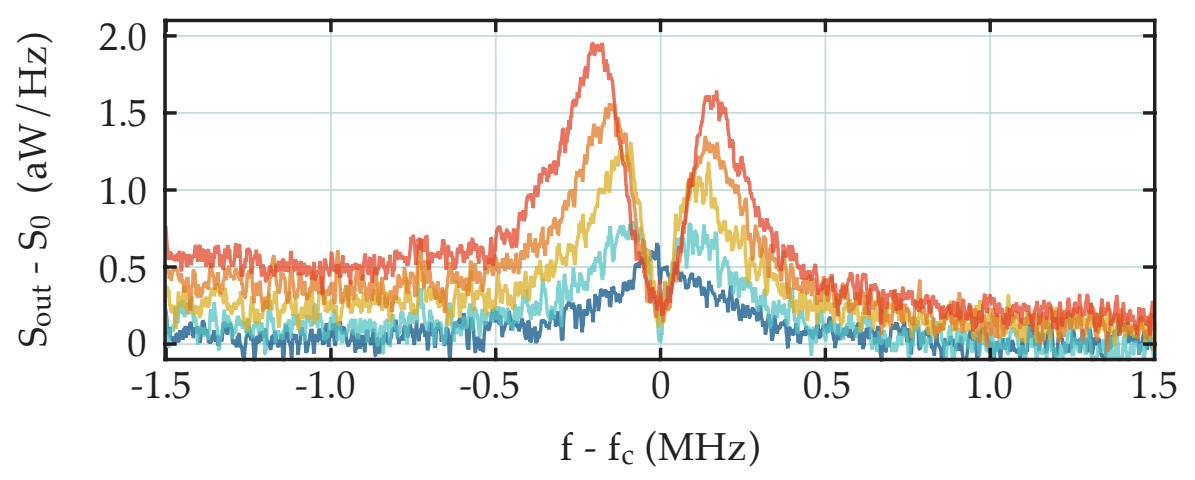

Figure 5.8: Cavity spectra for sideband cooling, with pump power increasing from 2.4e6 (blue) to $2.4 \mathrm{e} 7$ (red). The noise floor becomes more sloped as the power increases.

noise will couple into the cavity and can interact with the cavity and mechanics. If it is a source that occurs after the circulators on the device output line, then the noise does not interact with the mechanics. In either case, we will want to get rid of the source of the noise, but in the latter case, we can calibrate off the noise if necessary.

Our first assumption is that the noise is phase noise on the source that we have failed to filter sufficiently. We observed insufficient pump filtering before in past devices, however, and the frequency-dependence of the noise had a different shape, as seen in Fig. 5.9. To rule out phase noise on the pump, we add in a rejection filter centered at $\omega_{c}$ directly after the microwave source and compare to the un-filtered noise. We also tune the filter off of the cavity center and measure the noise. In both cases, the noise spectrum is the same as with no filtering, as shown in Fig. 5.10. If the noise were coming from the source, we'd expect the filter to create a dip in the floor. Since decreasing the pump noise does not change the output noise, we conclude that the noise must be added somewhere further along our measurement chain.

We switch to the through channel in the fridge and observe the same excess noise at the same output power, so we conclude that the noise does not originate in the device itself. We thus turn our attention to room-temperature contributions on the output line. To measure the powerdependent noise contributions at room temperature, we connect our filtered pump directly to the room-temperature output line, bypassing the fridge. The two possible contributors of excess noise in the room-temperature output line are the MiTeq low-noise amplifier (LNA) and the spectrum analyzer (SA). In between the source and each of these components, there are some losses. The system can be modeled as a chain of attenuators and amplifiers, as shown in Fig. 5.11. Each component has some gain, $G$, which is $>1$ for the amplifier and spectrum analyzer, and $<1$ for the attenuators. Each component also has some noise temperature, $T$. The attenuators' noise temperature is simply the temperature of the room, $T_{0} \approx 300 \mathrm{~K}$. According to their specifications, the LNA has a noise temperature of $T_{L N A} \approx 120 \mathrm{~K}$, and the spectrum analyzer has a noise temperature of $T_{S A} \approx 2000$ 


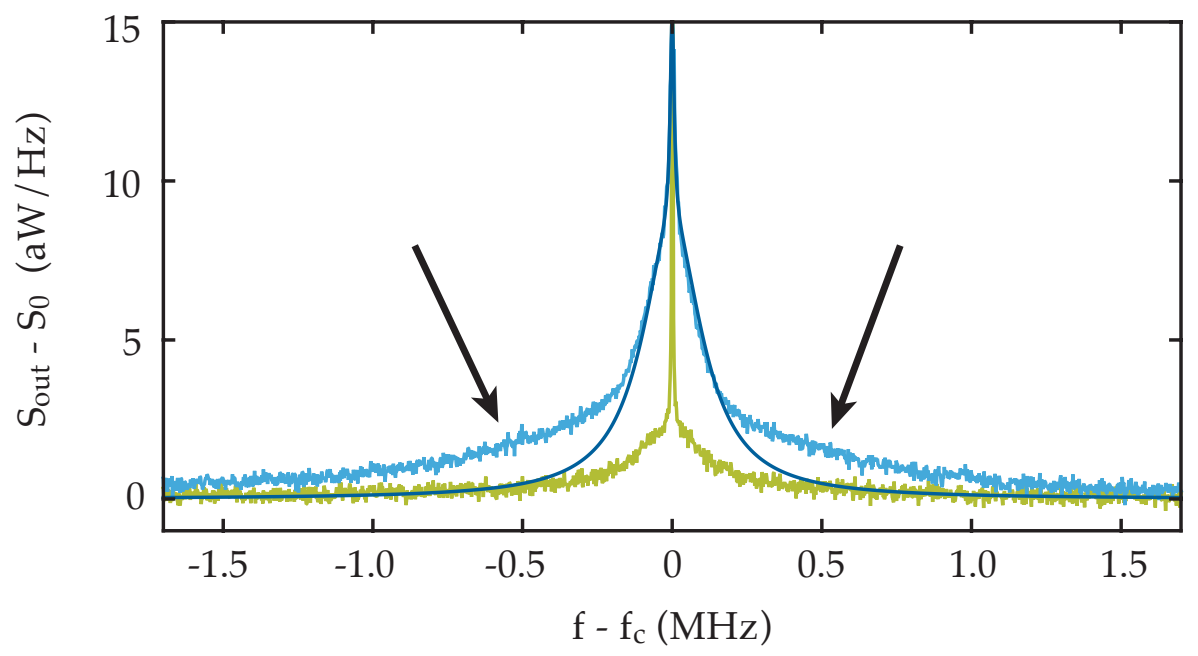

Figure 5.9: Signatures of insufficient source phase-noise filtering in a prior device. The light blue spectrum is taken at high pump powers, while the green spectrum is taken at low pump powers. The dark blue line is a fit to the high-power spectrum, with the floor fixed at 0 . Arrows point to regions where there is excess noise not included in the model. The source's phase noise is apparent because the source is filtered with a notch filter centered at $\omega_{c}$, but with a bandwidth comparable to the cavity bandwidth. The phase noise is thus suppressed at $\omega_{c}$ by the notch filter, and suppressed far from $\omega_{c}$ due to filtering by the cavity, but is evident at intermediate frequencies.

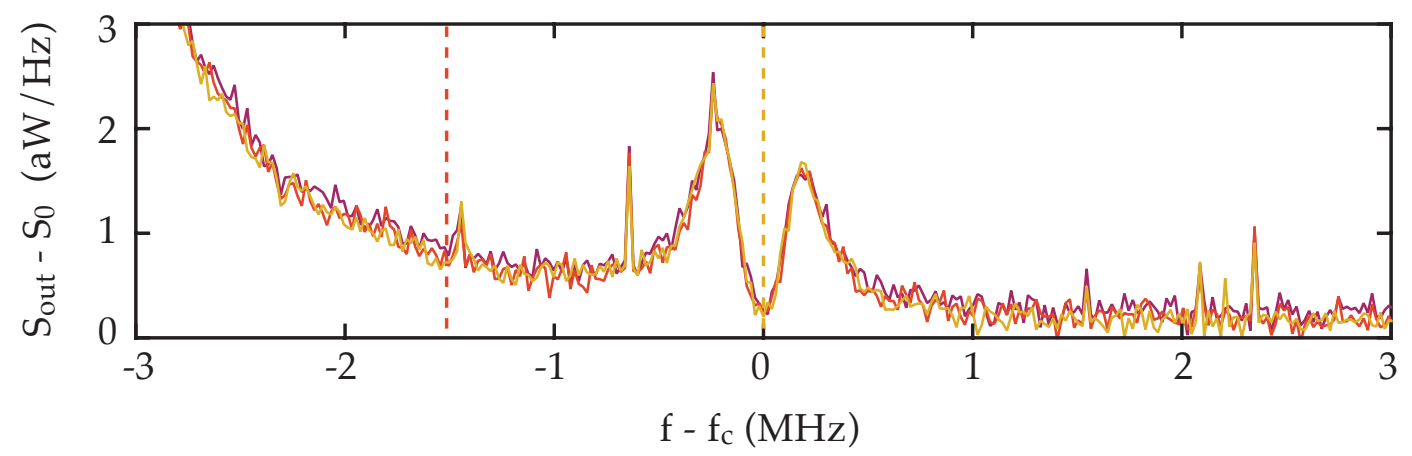

Figure 5.10: Cavity noise with no additional filtering of the pump (purple), with a rejection filter centered on the cavity (yellow), and with a rejection filter detuned from the cavity (red). Dashed lines indicate filter center frequencies. The filter has more than $30 \mathrm{~dB}$ of attenuation at its center frequency. No differences are observed between the spectra, within the noise level of the measurement. 


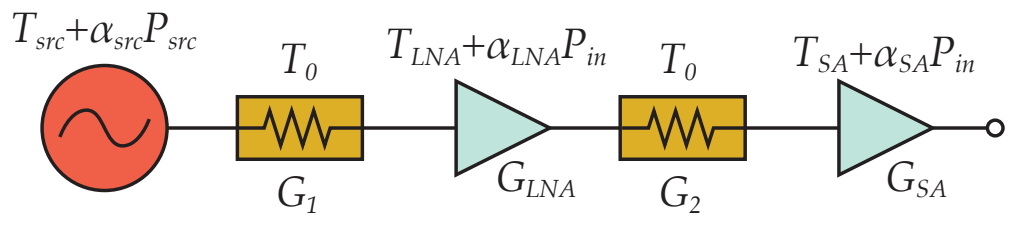

Figure 5.11: Model of room-temperature output line noise sources, showing the noise added by each component (above) and the gain of the component (below).

K. We can also include a hypothetical power-dependent noise term for both active components. For ideal operation, $\alpha_{L N A}=\alpha_{S A}=0$. The source also has some phase noise proportional to the source power, which we treat as a power-dependent noise temperature.

For now, we will assume that the gain of each component is independent of frequency. We can always compensate for differences in gain by measuring the frequency-dependence of the chain's transmission and applying a correction. We have made no assumptions about whether the powerdependence of the added noise is a function of frequency. In terms of the noise temperature of a given frequency bin $(T)$ or the coherent pump power $(P)$ arriving at the left port of each component, the output noise and power at the right port is given by

$$
\begin{aligned}
T_{\text {out }}^{\text {att } 1} & =G_{1} T_{\text {in }}+\left(1-G_{1}\right) T_{0} & P_{\text {out }}^{\text {att } 1} & =G_{1} P_{\text {in }} \\
T_{\text {out }}^{L N A} & =G_{L N A}\left(T_{\text {out }}^{a t t 1}+T_{L N A}+\alpha_{L N A} P_{\text {out }}^{\text {att } 1}\right) & P_{\text {out }}^{L N A} & =G_{L N A} P_{\text {out }}^{a t t 1} \\
T_{\text {out }}^{\text {att } 2} & =G_{2} T_{\text {out }}^{L N A}+\left(1-G_{2}\right) T_{0} & P_{\text {out }}^{\text {att } 2} & =G_{2} P_{\text {out }}^{L N A} \\
T_{\text {out }}^{S A} & =G_{S A}\left(T_{\text {out }}^{\text {att } 2}+T_{S A}+\alpha_{S A} P_{\text {out }}^{\text {att } 2}\right) & P_{\text {out }}^{S A} & =G_{S A} P_{\text {out }}^{\text {att } 2} .
\end{aligned}
$$

So, for the whole chain, we obtain

$$
\begin{aligned}
T_{\text {out }}= & G_{S A} G_{2} G_{L N A} G_{1} T_{i n}+\left[G_{S A} G_{2} G_{L N A}\left(1-G_{1}\right)+G_{S A}\left(1-G_{2}\right)\right] T_{0} \\
& +G_{S A} G_{2} G_{L N A} T_{L N A}+G_{S A} G_{2} G_{L N A} G_{1} \alpha_{L N A} P_{i n} \\
& +G_{S A} T_{S A}+G_{S A} G_{2} G_{L N A} G_{1} \alpha_{S A} P_{i n} .
\end{aligned}
$$

To calculate $T_{\text {out }}$ when the source is on, we set $P_{i n}=P_{s r c}$ and $T_{i n}=T_{s r c}+\alpha_{s r c} P_{s r c}$. When the source is off, $P_{i n}=0$ and $T_{i n}=T_{0}$. The difference between the output temperature when the source is on and when it is off is then

$$
\Delta T_{\text {out }}=G_{S A} G_{2} G_{L N A} G_{1}\left(T_{s r c}+\alpha_{s r c} P_{s r c}-T_{0}+\alpha_{L N A} P_{s r c}+\alpha_{S A} P_{s r c}\right) .
$$

If we measure the total gain $G_{t o t}=G_{S A} G_{2} G_{L N A} G_{1}$, we can plot $\Delta T_{\text {out }}$ vs. $G_{t o t} P_{s r c}$. The resulting plot should be linear with an offset of $G_{t o t}\left(T_{s r c}-T_{0}\right)$ and a slope of $\alpha_{s r c}+\alpha_{L N A}+\alpha_{S A}$. If we filter 


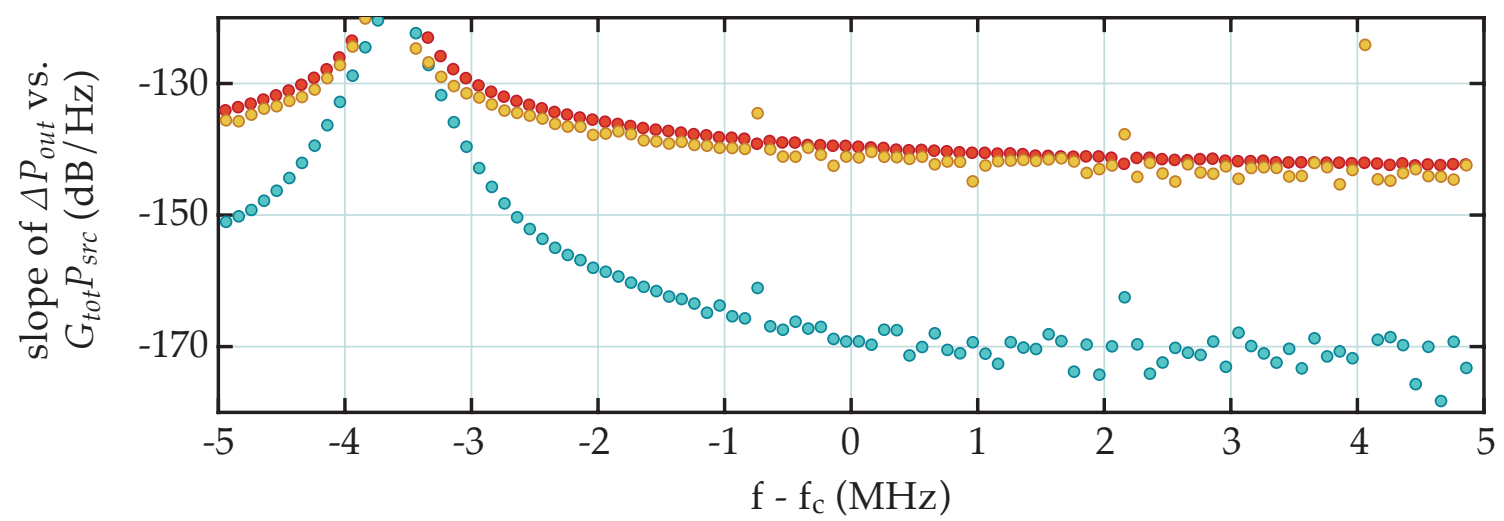

Figure 5.12: Results of room-temperature filtering on the output line phase noise. The slope of $\Delta P_{\text {out }}$ vs. $G_{t o t} P_{s r c}$ is plotted for no filtering (red), filtering before the spectrum analyzer (yellow), and filtering before the LNA (turquoise). $\Delta P_{\text {out }} \propto \Delta T_{\text {out }}$ is the measured noise power spectral density. Each circle represents a $100 \mathrm{kHz}$ frequency bin for which an individual fit of $\Delta P_{\text {out }}$ vs. $G_{t o t} P_{s r c}$ was performed. Filtering before the spectrum analyzer produces little change to the slope, while filtering before the LNA decreases the noise by up to $25 \mathrm{~dB}$. Note that the pump noise slope is reduced below the $\sim-145 \mathrm{dBc} / \mathrm{Hz}$ that we'd expect at $3.6 \mathrm{MHz}$ away from the carrier due to a filter cavity in transmission centered at $\omega_{-}=\omega_{c}-\omega_{m}$ directly after the source.

the pump frequency before the spectrum analyzer, $P_{s r c}$ will be multiplied by a factor of $G_{f i l t}<1$ before entering the SA, and the slope of $\Delta T_{\text {out }}$ vs. $G_{t o t} P_{s r c}$ becomes $\alpha_{s r c}+\alpha_{L N A}+G_{f i l t} \alpha_{S A}$. This suppresses the contribution from the power-dependent noise due to the spectrum analyzer. If the spectrum analyzer is adding excess noise in the presence of the pump, we should see a decrease in the slope when the pre-SA filter is in place. Similarly, if we put a filter in front of the LNA, the slope becomes $\alpha_{s r c}+G_{f i l t} \alpha_{L N A}+G_{f i l t} \alpha_{S A}$, and we suppress the contributions from both the LNA and the spectrum analyzer. By comparing the slopes for the no-filter, pre-SA filter, and pre-LNA filter cases, we are able to tell if one of the circuit components is adding power-dependent noise.

The slope vs. frequency is shown for these three cases in Fig. 5.12. We see that filtering before the spectrum analyzer does not change the power-dependence of the noise, while filtering before the LNA decreases the noise by more than $20 \mathrm{~dB}$ for frequencies near $\omega_{c}$. This suggests that the LNA is adding phase noise proportional to the pump power, even when the incident power is well below the LNA's compression point (as it is for all pump powers discussed in this chapter). We tried replacing the LNA with a similar MiTeq amplifier, but we observed the same power-dependent noise.

As using a filter on the output line increases losses at the cavity frequency, we instead decide to cancel out the pump power at the output of the fridge, before the LNA. A schematic for the cancellation circuit is shown in Fig. 5.13, and a comparison of the power dependence of the noise for cancellation before and after the LNA is shown in Fig. 5.14. The cooling data taken in Section 5.2.4 was taken with the pre-LNA cancellation circuit in place. No power-dependent noise slope was observed after cancellation. 


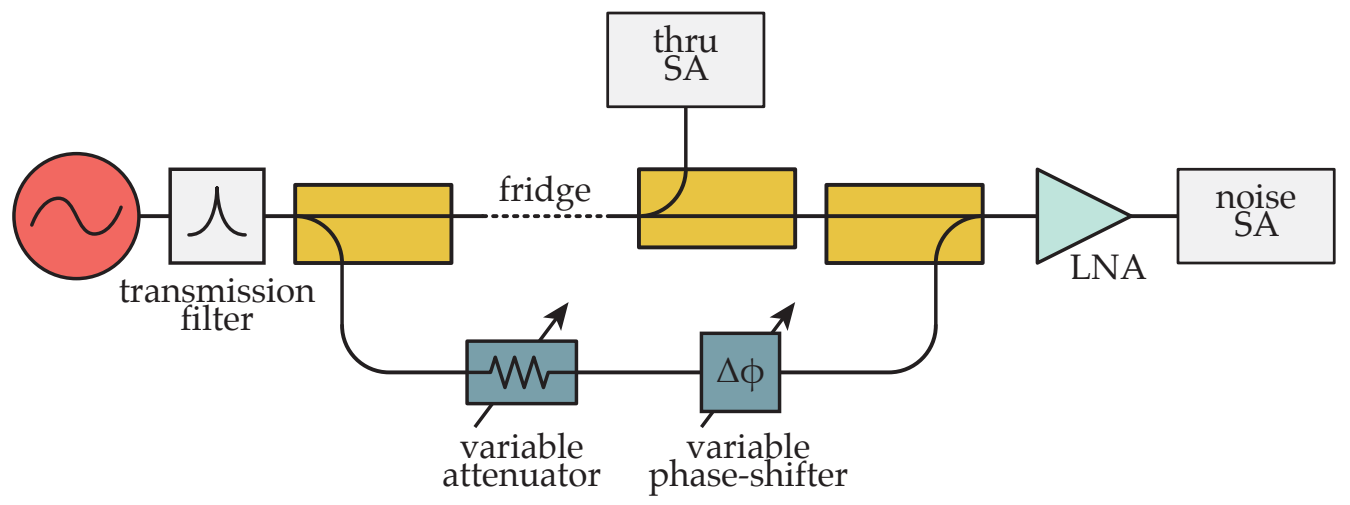

Figure 5.13: Circuit diagram for pump cancellation. Some of the filtered pump power is split off before the pump enters the fridge. The tone is attenuated and phase-shifted so that it destructively interferes with the pump at the output of the fridge. Pump through powers are measured on a second spectrum analyzer prior to cancellation to avoid any drifts in through power due to changes in the cancellation line. Cancellation of more than $30 \mathrm{~dB}$ is achievable on time scales of 12 hours.

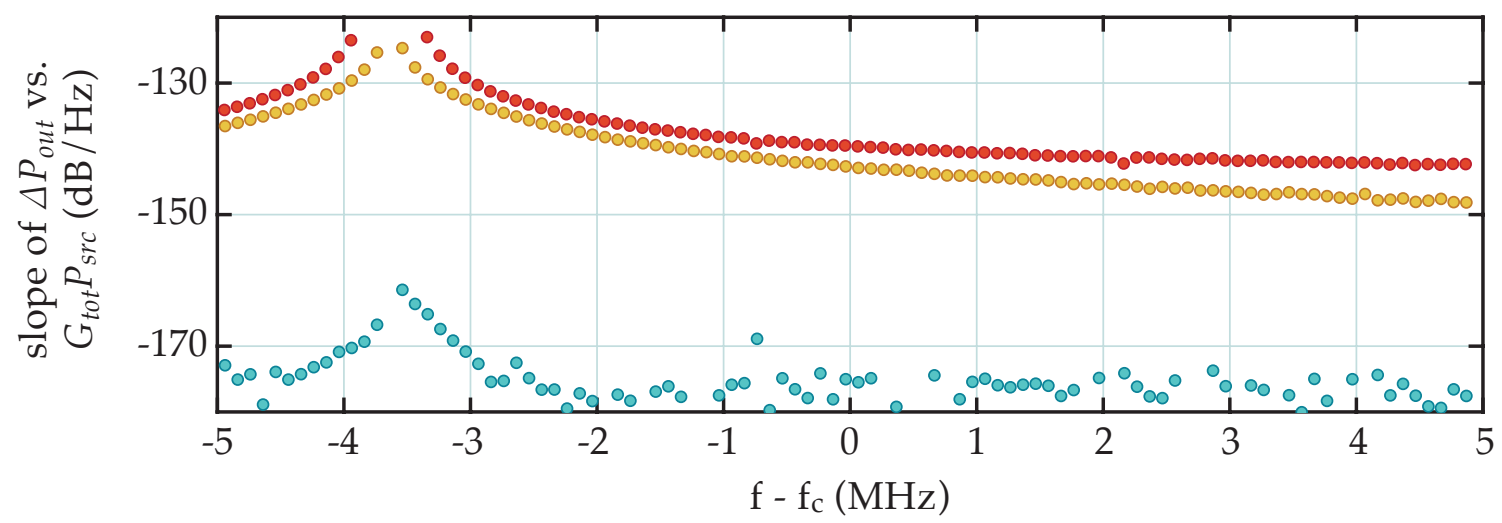

Figure 5.14: Results of room-temperature cancellation on the output line phase noise. The slope of $\Delta P_{\text {out }}$ vs. $G_{t o t} P_{\text {src }}$ is plotted for no cancellation (red), cancellation before the spectrum analyzer (yellow), and cancellation before the LNA (turquoise). Analysis is the same as that for Fig. 5.12. Cancellation before the spectrum analyzer produces a small change to the slope, while filtering before the LNA decreases the noise by about $30 \mathrm{~dB}$. 


\subsection{Squeezing analysis}

\subsubsection{Procedure}

To squeeze the mechanics, we first set up squeezing pumps at $\omega_{c} \pm \omega_{m}$ and measure the through powers of the pumps. We then measure driven responses with spans on the scale of the cavity linewidth and on the scale of the mechanical linewidth. We use Eq. 2.43 to fit both the real and imaginary parts of the driven response transmission. The calibrations from Section 5.2.2 are used to determine $G_{-}$and $G_{+}$. The cavity response is fitted first, with free parameters for $\omega_{c}, \kappa$, and a linear, complex floor. Estimates for $\gamma_{m}$ and $\omega_{m}$ are entered as fixed parameters. Once $\omega_{c}$ and $\kappa$ are determined, the mechanical response is fitted to obtain $\omega_{m}$. At the high pump powers used for squeezing, the mechanical linewidth is much greater than $\gamma_{m}$, and so it can be ignored in the fitting. The results of this fitting procedure are shown in Fig. 5.15.

If needed, the fit can be iterated using the previous fit's results as estimates for the new fit. As the driven response fit model includes the effects of imperfect alignment (i.e., it does not make the assumption $\Delta=\delta=0$ ), the driven response fits can be used to align the pump frequencies. We can measure the driven response to obtain $\omega_{m}$ and $\omega_{c}$, adjust the pump frequencies, and repeat the driven response measurement until our pumps are positioned at $\omega_{c} \pm \omega_{m}$. Alternatively, we can sweep the detuning as described in Section 5.3.3.

Once the pumps are aligned, we switch from driven responses to spectral measurements. As with D1, we take data in 5 minute averaging windows, interleaving spectra with the squeezing pumps on and floors with the pumps off. We measure the through powers of the squeezing pumps before every pumps-on spectrum. Sample spectra after 5 minutes of averaging are shown in the top-left panel of Fig. 5.16. We take between 10 and 25 of these spectra for each pump configuration. The average of 25 5-minute spectra is shown in the top-right panel of Fig. 5.16. Here, the kink in the noise floor is clearly evident. This kink is most-likely due to frequency dependence in the noise floor of our measurement system, as discussed in Section 5.2.3. Note also that the floor of the pumps-on spectrum appears to be more sloped than the pumps-off noise floor. This is because we are not implementing the pump-cancellation scheme in Fig. 5.13 to reduce the noise added by the room-temperature LNA, as discussed in Section 5.2.5. The squeezing data presented here were taken with both squeezing pumps generated by an Agilent E8267C vector source, which was found to produce better squeezing than using two separate sources for the squeezing pumps. The vector source, however, is difficult to cancel, as the tones cannot be phase-shifted and attenuated individually. As we found that the excess noise comes from a source further along the measurement line that cannot possibly interact with the cavity due to our cryogenic circulators, we can subtract it out of the spectrum. The excess noise contribution is linear across the linewidth of the cavity, so we perform a linear fit on the wings of our measured background-subtracted spectra and subtract off this contribution. A background- 
cavity
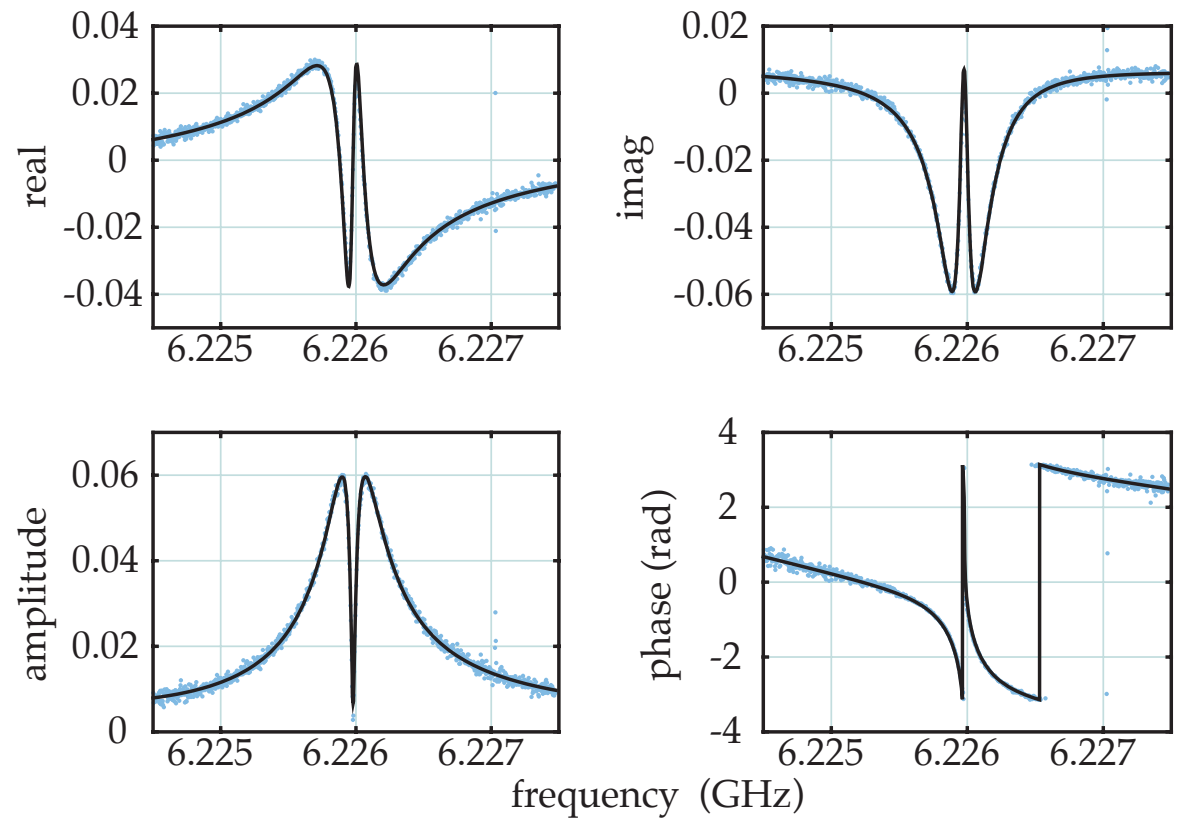

mechanics
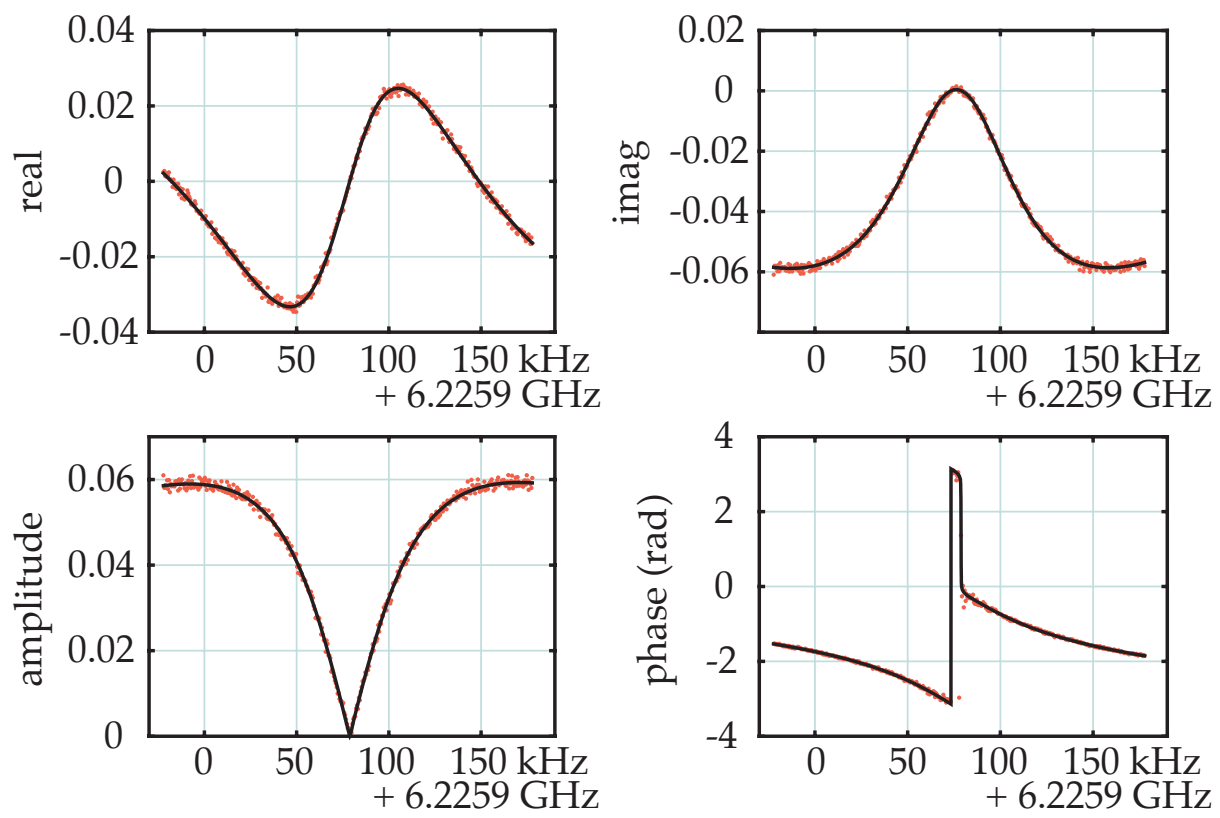

frequency

Figure 5.15: Driven response fitting for squeezing measurements. The cavity response (top) is fitted first, with a fixed estimate for the mechanical frequency. The mechanical response (bottom) can then be fitted to find $\omega_{m}$. The fits are in good agreement with the measured transmission responses. 

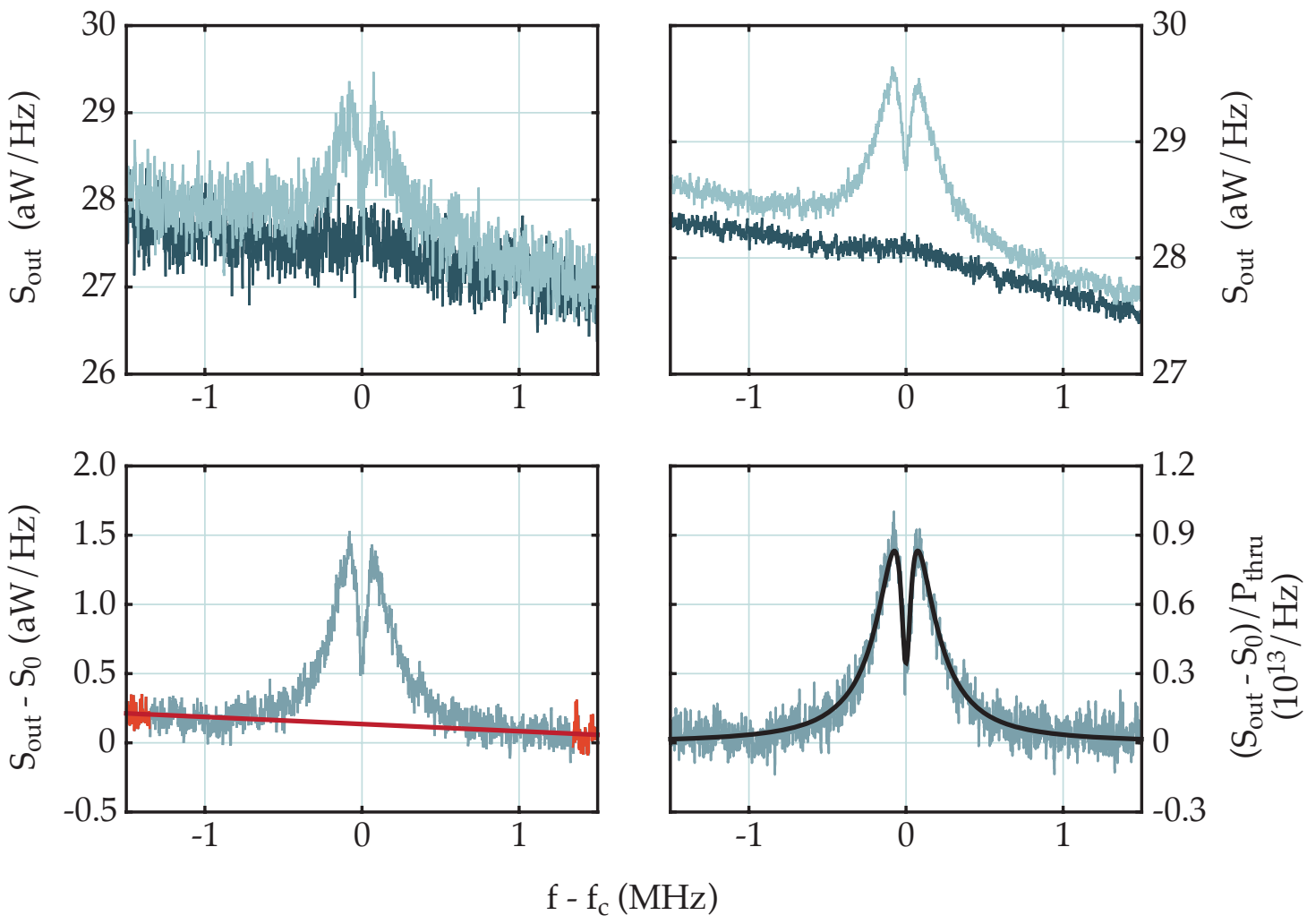

Figure 5.16: Data processing and analysis for squeezing spectra. Top left: 5-minute averages of the pumps-on spectrum (light) and pumps-off floor (dark). Top right: 25 5-minute spectra averaged together for the squeezing spectrum (light) and floor (dark). The kink in the noise floor is evident. Bottom left: Background-subtracted, averaged spectrum. There still appears to be a slight slope in the noise floor due to the noise added by the LNA, as discussed in Section 5.2.5. To subtract off this noise added by the output chain, we apply a linear fit to the portions of the spectrum shown in red. Bottom right: Spectrum with linear background subtracted, normalized by the red-detuned tone's through power. The black line is a fit to Eq. 5.10.

subtracted spectrum showing the linear background fit is shown in the bottom-left panel of Fig. 5.16. Lastly, any spurs are removed from the spectrum, and the spectrum is binned.

We normalize the background-subtracted spectrum by $P_{t h r u}^{-}$to cancel out the factor of $\kappa_{R}$ in Eq. 2.54, so that the measured spectrum can be written as

$$
\left(S_{\text {out }}-S_{0}\right) / P_{t h r u}^{-}=\frac{1}{\left(1+\xi \omega_{m}\right)^{2} n_{p}^{-}}\left\{\frac{\omega^{2} \kappa n_{c}^{t h}+\left(G_{-}^{2}+G_{+}^{2}\right) \gamma_{m} n_{m}^{t h}}{\left|\mathcal{G}^{2}-i \omega(\kappa / 2-i \omega)\right|^{2}}\right\}
$$

centered at $\omega=\omega_{c}$. In Section 2.6.1, we made the assumptions that $\gamma_{m} \ll \mathcal{G}, \kappa, n_{c, R}^{t h}=0$, and $n_{m}^{t h} \gg 1$ in deriving the output spectrum from which Eq. 5.9 is derived. All these conditions are met for the measurements described here. As we don't have a good way to measure $\gamma_{m}$ in the presence of the large pump powers, we combine the factor of $\gamma_{m}$ in the output spectrum with $n_{m}^{t h}$, and simply 
fit for the mechanical loss rate, $\dot{n}_{m}^{t h}=\gamma_{m} n_{m}^{t h}$. We then have

$$
\left(S_{\text {out }}-S_{0}\right) / P_{\text {thru }}^{-}=\frac{1}{\left(1+\xi \omega_{m}\right)^{2} n_{p}^{-}} \frac{\omega^{2} \kappa n_{c}^{t h}+\left(G_{-}^{2}+G_{+}^{2}\right) \dot{n}_{m}^{t h}}{\left|\mathcal{G}^{2}-i \omega(\kappa / 2-i \omega)\right|^{2}} .
$$

We use values for $G_{-}, G_{+}, n_{p}^{-}$, and $\xi \omega_{m}$ obtained from calibrations for the measured red- and bluedetuned pump through powers (Sections 5.2.1 and 5.2.2). $\kappa$ is obtained from the driven response fit described above. The only free parameters are then $n_{c}^{t h}, \dot{n}_{m}^{t h}$, and a linear offset. An example of a spectral fit is shown in the bottom-right panel of Fig. 5.16.

Once the bath occupations are known, we can estimate the quadrature variances using Eqs. 2.38 and 2.39. In the limit where $\gamma_{m} \ll \mathcal{G}, \kappa$ and $n_{m}^{t h} \gg 1$, these equations become

$$
\begin{aligned}
\left\langle\hat{X}_{1}^{2}\right\rangle & =\frac{\left(G_{-}-G_{+}\right)^{2}}{\mathcal{G}^{2}}\left(n_{c}^{t h}+1 / 2\right)+\frac{4 \mathcal{G}^{2}+\kappa^{2}}{4 \mathcal{G}^{2}} \dot{n}_{m}^{t h} / \kappa \\
\left\langle\hat{X}_{2}^{2}\right\rangle & =\frac{\left(G_{-}+G_{+}\right)^{2}}{\mathcal{G}^{2}}\left(n_{c}^{t h}+1 / 2\right)+\frac{4 \mathcal{G}^{2}+\kappa^{2}}{4 \mathcal{G}^{2}} \dot{n}_{m}^{t h} / \kappa .
\end{aligned}
$$

\subsubsection{Parameter space search}

Based on the bath occupations vs. pump power for the cooling experiment in Section 5.2.4, we find that optimal squeezing is predicted for total pump powers over $n_{p}^{-}+n_{p}^{+}=10^{7}$, and for $n_{p}^{+} / n_{p}^{-}$ ratios above 0.5 (Fig. 5.7). We thus focus our search of parameter space on these regions. The fit results for total pump powers between $1 \mathrm{e} 7$ and $2 \mathrm{e} 7$, and for ratios between 0.52 and 0.72 are shown in Fig. 5.17.

We find that optimum squeezing is produced for total pump powers of $1.75 \times 10^{7}$ and $2.0 \times 10^{7}$, and for ratios near the lower end of the parameter space that we measured. We choose to focus on $n_{p}^{-}+n_{p}^{+}=1.75 \times 10^{7}$. We also find our first indication of ratio-dependent heating, as the cavity bath occupation is observed to increase slightly with increasing pump ratio.

\subsubsection{Alignment}

To produce optimal squeezing, it is important to align the pumps so that they are positioned at exactly $\omega_{c} \pm \omega_{m}$. As seen in Section 2.4.4, changes in $\delta$ on the scale of $1 \%$ of the mechanical linewidth can produce a noticeable effect on the amount of squeezing, while changes in $\Delta$ on the scale of $10 \%$ of the cavity linewidth can start to affect the squeezing. As we can measure the cavity frequency well within $0.1 \times \kappa$, we focus our alignment procedure on ensuring that $\delta=0$.

From our model of the driven response (Section 2.5, and in particular the center column of Fig. 2.5), we see that, when $\delta \neq 0$, the mechanical dip is shallower than when $\delta=0$, and is not centered between the two pump frequencies. We thus set up our squeezing tones at $\omega_{c} \pm\left(\omega_{m}+\delta\right)$ 

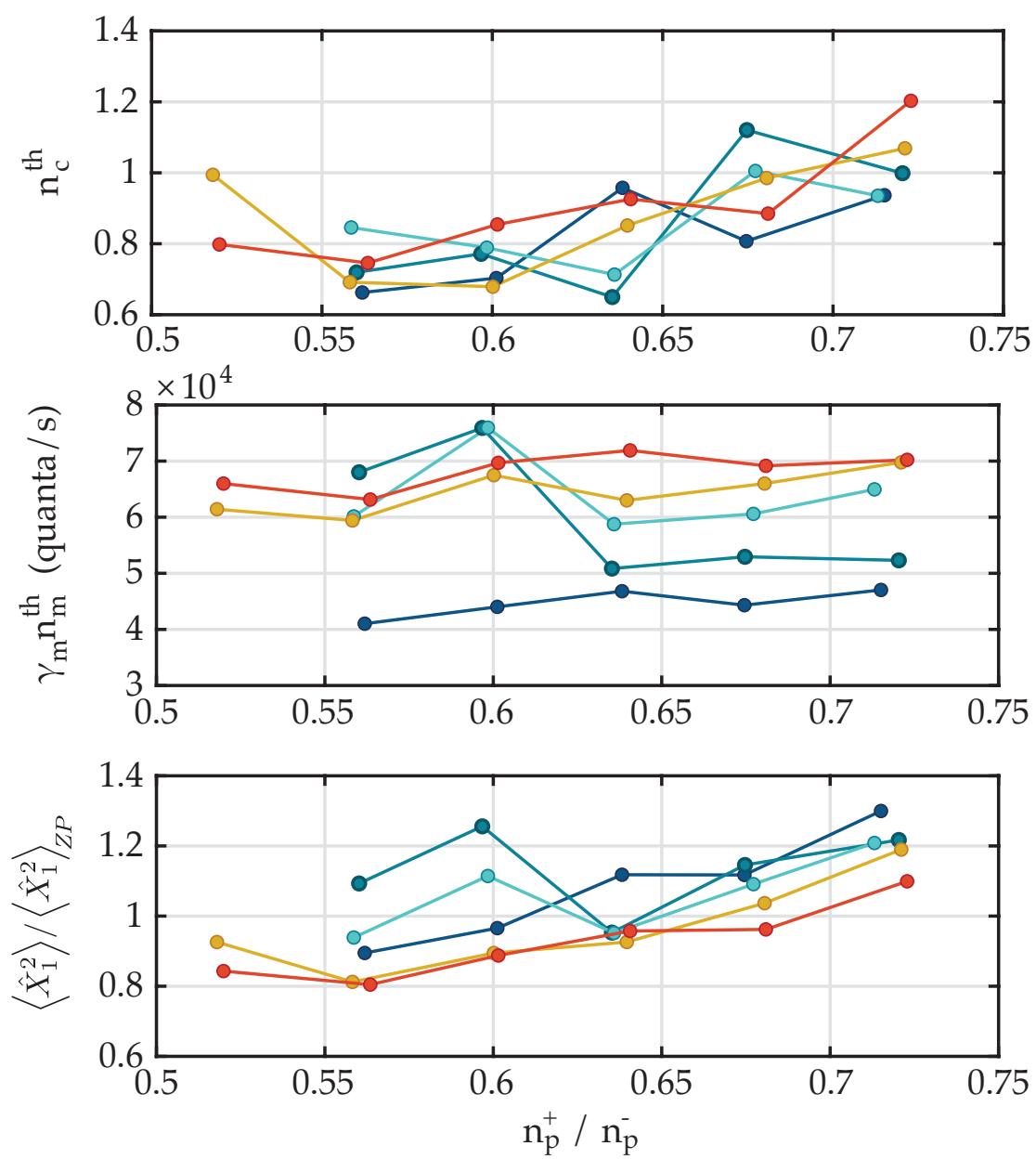

Figure 5.17: Bath occupations and $\hat{X}_{1}$ quadrature variance vs. pump power ratio for total pump powers ranging from $1.0 \times 10^{7}$ (dark blue) to $2.0 \times 10^{7}$ (red) in steps of $0.25 \times 10^{7}$. Error bars $(\sim>5 \%)$ are not shown for this rough search. While $n_{c}^{t h}$ does not vary strongly with pump power over this small range, there is a slight trend of increasing $n_{c}^{t h}$ with increasing ratio evident for all pump powers. The mechanical bath is observed to heat up monotonically with increasing pump power for all ratios apart from $n_{p}^{+} / n_{p}^{-} \sim 0.55$ and 0.6. Optimum squeezing is produced for the higher pump powers at the lowest ratios. 

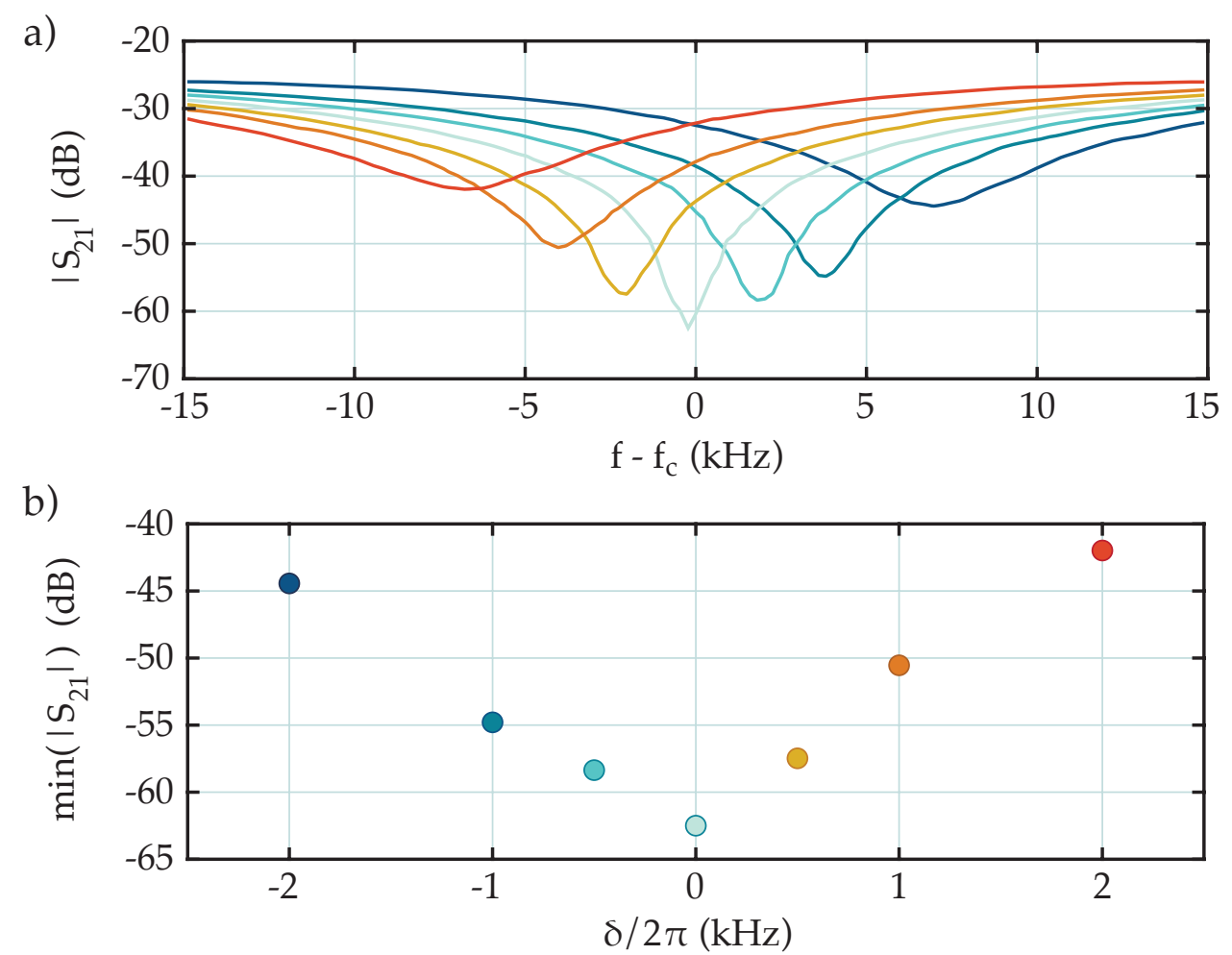

Figure 5.18: Frequency alignment for squeezing tones. a) Mechanical driven response for detunings between $-2 \pi \times 2 \mathrm{kHz}$ (red) and $2 \pi \times 2 \mathrm{kHz}$ (blue). The transmission at $\omega_{c}$ appears to be minimized somewhere between the $-2 \pi \times 500 \mathrm{~Hz}$ and $2 \pi \times 0 \mathrm{~Hz}$ detunings. b) Minimum transmission vs. detuning. The detuning can be determined to less than $500 \mathrm{~Hz}$.

and try varying $\delta$ until we minimize the transmission at $\omega_{c}$. An example of this alignment procedure is shown in Fig. 5.18. Using this procedure, we can ensure $\delta<2 \pi \times 500 \mathrm{~Hz}$.

\subsubsection{Fit results: ideal assumptions}

After choosing red- and blue-detuned pump powers and aligning the pumps, we measure and fit the driven and spectral responses assuming perfect alignment and no bad-cavity effects, as described in Section 5.3.1. We repeat this procedure at $n_{p}^{-}+n_{p}^{+}=1.76 \times 10^{7}$ for different $n_{p}^{+} / n_{p}^{-}$pump ratios. Examples of fits to the driven and spectral responses are shown in Fig. 5.19. From the spectral fits, we obtain the bath occupations, $n_{m}^{t h}$ and $n_{c}^{t h}$, as shown in Fig. 5.20. Then, using Eq. 5.11, we can find the quadrature noise expectation values, again assuming perfect alignment and no bad-cavity effects, as shown in Fig. 5.21. Error bars are propagated from the fit errors for $\kappa, n_{c}^{t h}$, and $\dot{n}_{m}^{t h}$ and from the calibration errors for $G_{-}, G_{+}, \xi$, and $n_{p}^{-}$assuming non-correlated, Gaussian error distributions.

As seen in Fig. 5.21, several points are squeezed below the zero-point fluctuations, with a minimum measured quadrature variance of $\left\langle\hat{X}_{1}^{2}\right\rangle=0.81 \pm 0.03\left\langle\hat{X}_{1}^{2}\right\rangle_{Z P}$ at a pump power ratio of $\sim 0.4$. The squeezing degrades above a ratio of 0.6 , due to a sudden increase in $n_{c}^{t h}$ at higher ratios. 
a)

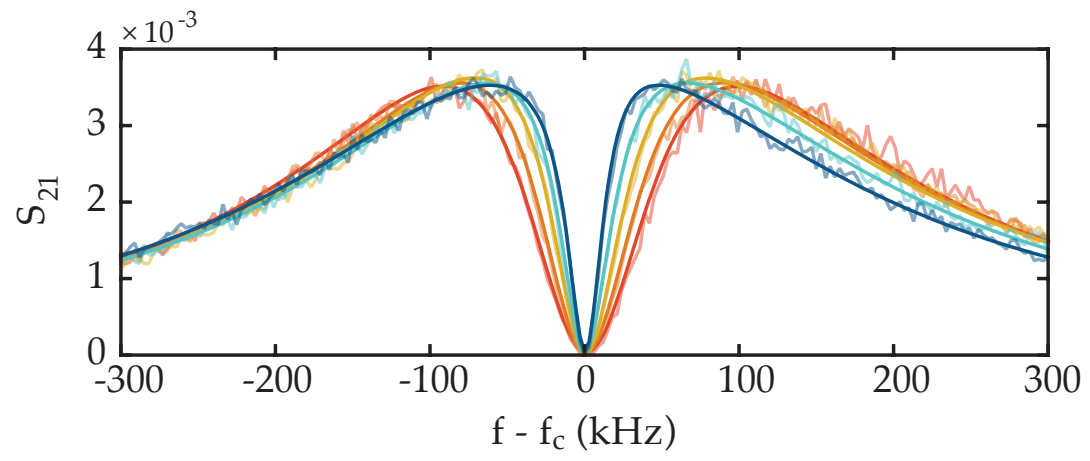

b)

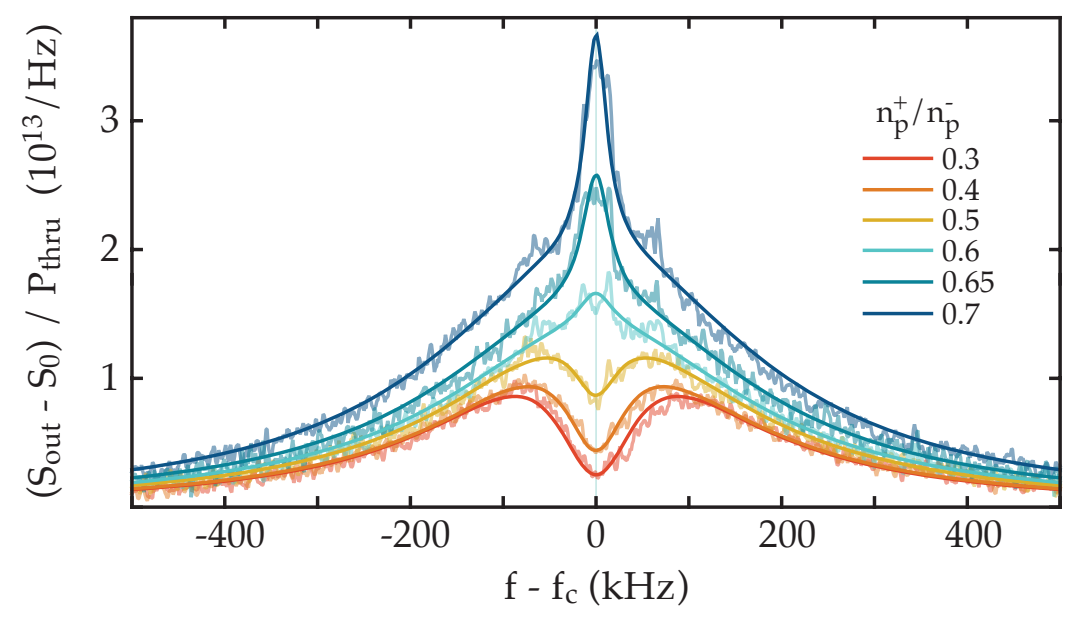

Figure 5.19: Fits of the squeezing model to both driven (a) and spectral (b) responses. The driven response model (Eq. 2.5) allows for imperfect detunings, while the spectral response model (Eq. 5.10) assumes that $\Delta=\delta=0$. Both assume negligible bad-cavity effects. As the $n_{p}^{+} / n_{p}^{-}$ratio increases, the effective damping decreases, and the mechanical linewidth narrows. In the spectral response, the mechanical response changes from a dip to a peak as the noise squashing effects decrease with decreasing red power. 


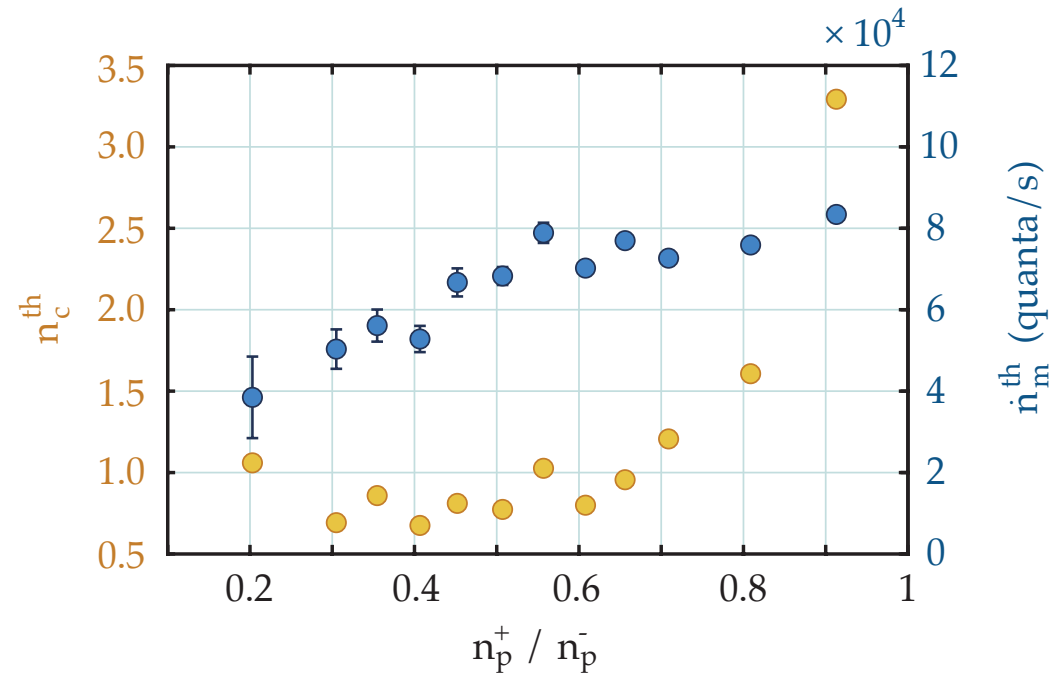

Figure 5.20: Quadrature variances vs. pump ratio. Left: both $\left\langle\hat{X}_{2}^{2}\right\rangle$ and $\left\langle\hat{X}_{1}^{2}\right\rangle$ vs. pump ratio. The shaded area indicates sub-zero-point squeezing. Right: Close-up of boxed region on the left. Several points are multiple error bars below the zero-point fluctuations.
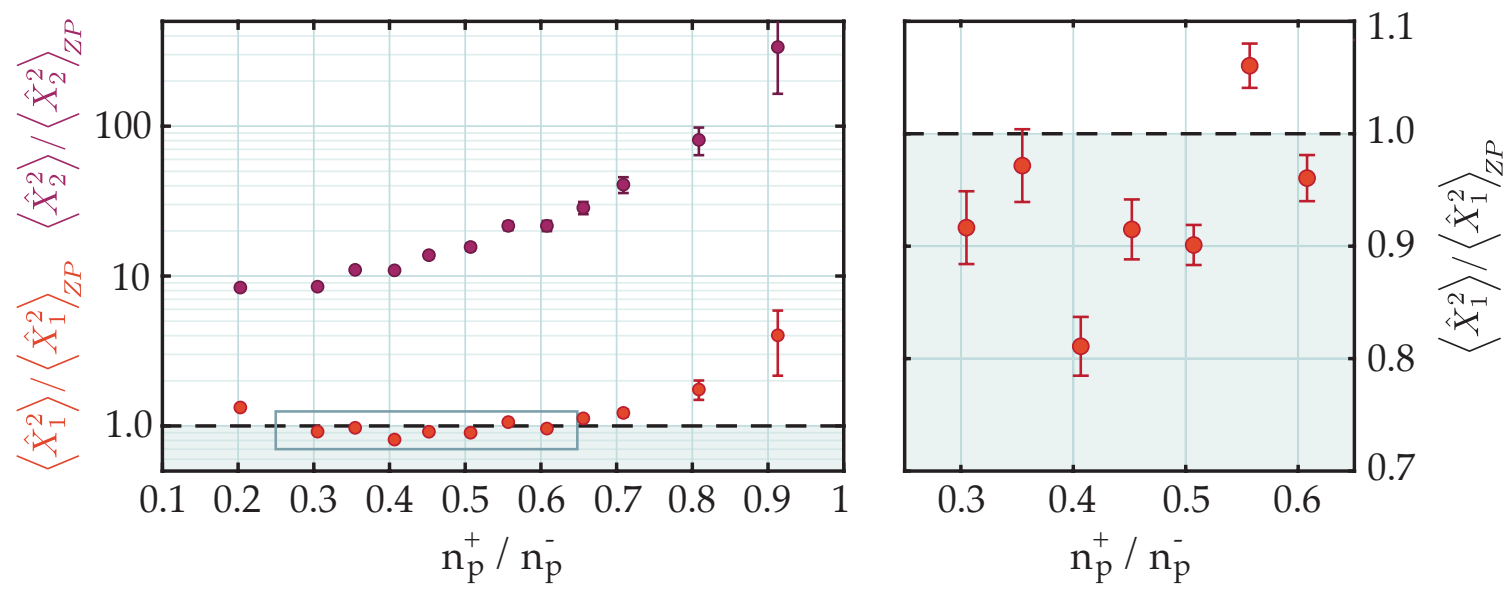

Figure 5.21: Quadrature variances vs. pump ratio. Left: both $\left\langle\hat{X}_{2}^{2}\right\rangle$ and $\left\langle\hat{X}_{1}^{2}\right\rangle$ vs. pump ratio. The shaded area indicates sub-zero-point squeezing. Right: Close-up of boxed region on the left. Several points are multiple error bars below the zero-point fluctuations. 
There are several assumptions made in obtaining the results of Fig. 5.21 that may not be generally true. First, we neglected the bad-cavity effects due to $\omega_{m} \gg \kappa$. In sideband cooling, neglecting these effects introduces errors on the order of $\left(\kappa / \omega_{m}\right)^{2}$, which is $1.5 \%$ for D2. Second, we assumed that the pumps are perfectly-aligned with $\Delta=\delta=0$. As seen in Fig. 2.4, imperfect alignment can degrade the amount of squeezing, particularly at high pump ratios. It also alters the appearance of the spectrum (Fig. 2.6). From our alignment measurements (Section 5.3.3), we expect to be aligned within $2 \pi \times 500 \mathrm{~Hz}$, but ideally we'd like to take the detuning into account in both fitting the spectrum and extracting the quadrature variances. Lastly, in calculating the error bars for the quadrature variances, we assumed that all our sources of error are uncorrelated. This is not necessarily the case - overestimating $n_{c}^{t h}$ may make the fit routine underestimate $\dot{n}_{m}^{t h}$ to compensate, for example. The next section addresses these assumptions.

\subsubsection{Fit results: including alignment and bad cavity effects}

It is possible to include the effects of imperfect alignment and of the first-order counter-rotating terms by using a numerical model to fit the squeezing spectra and extract the quadrature variances. To take into account the unknown correlations of our fit parameters in the error bars of the estimated quadrature variances, we use a Bayesian approach. This analysis is due to A. Weinstein, and is described in the supplementary information of [64], so I will only summarize the procedure here.

The ultimate goal of the Bayesian analysis is to find the posterior probability distribution of obtaining a set of bath occupations, $\alpha=\left\{n_{c}^{t h}, \dot{n}_{m}^{t h}\right\}$, as well as the set of supporting measurements, $\beta$, given the measured squeezing spectrum, $D$, and the full, non-RWA model of our system, $I$. Here, $\beta$ includes all calibrations (thermal calibration and linewidth broadening calibration slopes), measured frequencies obtained from the driven response $(\kappa, \Delta$, and $\delta)$, and the measured pump through powers $\left(P_{t h r u}^{-}\right.$and $\left.P_{t h r u}^{+}\right)$. We can then find the Bayesian posterior distribution by calculating

$$
p(\alpha, \beta \mid D, I)=p(D \mid \alpha, \beta, I) p(\alpha, \beta) / p(D),
$$

where $p(D \mid \alpha, \beta, I)$ is the likelihood function for obtaining the measured spectrum given $\alpha, \beta$, and the system model, $p(\alpha, \beta)$ is the prior distribution for $\alpha$ and $\beta$, and $p(D)$ is an overall scale constant that does not affect the posterior distribution peak or width. We take the prior distributions for $\beta$ to be independent Gaussian distributions, and assume that the priors for $\alpha$ are uniform in log space.

The posterior distribution is sampled numerically using the emcee package in Python [17]. The resulting distribution can then be used to construct the full, non-RWA quadrature spectrum, $S_{X_{1,2} X_{1,2}}$, and the quadrature distributions can be found via numerical integration.

The results of numerical fitting are shown in Fig. 5.22, and the estimated bath occupations and quadrature variances are shown in Fig. 5.23, with the ideal model values shown for comparison. The 

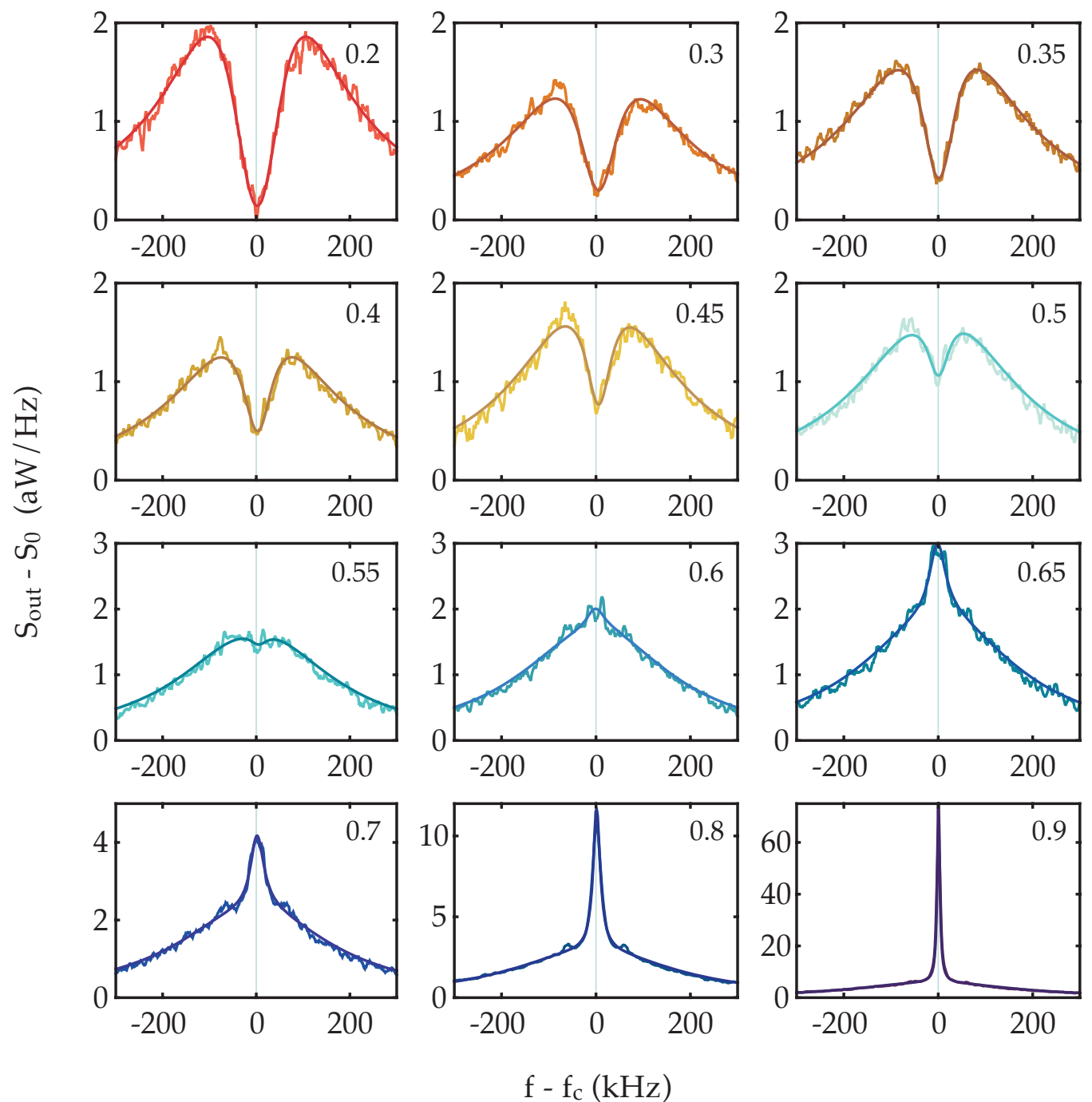

Figure 5.22: Full-model spectral fits for all measured ratios. The pump power ratio $n_{p}^{+} / n_{p}^{-}$is displayed in the upper-right corner of each plot.

minimum quadrature noise obtained with the full analysis is $\left\langle\hat{X}_{1}^{2}\right\rangle=0.80 \pm 0.03\left\langle\hat{X}_{1}^{2}\right\rangle_{Z P}$ at a ratio of 0.4 .

In general, the two analysis approaches agree within their error bars at pump ratios at or below 0.5 , and then begin to deviate at higher ratios, with some full-model points having lower values of $\left\langle\hat{X}_{1}^{2}\right\rangle$ than the simple-simple model fits, and some having higher. While both models extract similar values for $n_{c}^{\text {th }}$ (apart from at a ratio of 0.55 , which appears to be an outlier for the simple model), the models differ in their extracted $\dot{n}_{m}^{t h}$ values. This is likely due to the inclusion of imperfect detuning in the full model: imperfect alignment can have a large effect on the mechanical response in the output spectrum (see Fig. 2.6), and thus on the estimation of $\dot{n}_{m}^{t h}$, but has less of an effect on the cavity response. The error bars for $\left\langle\hat{X}_{1}^{2}\right\rangle$ from the Bayesian analysis also differ from those of the 

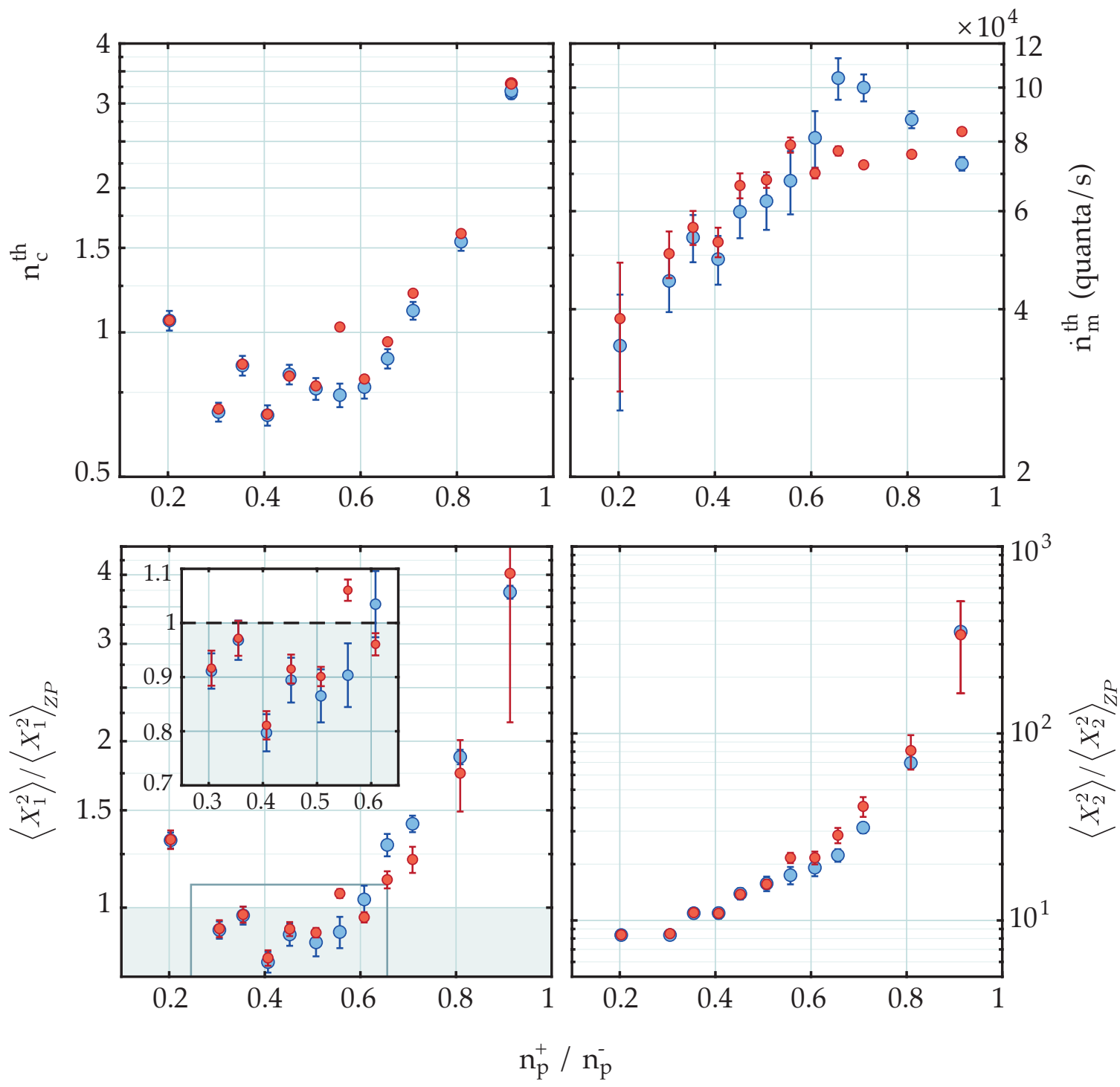

Figure 5.23: Comparison of the results of a full Bayesian analysis, including frequency detunings and counter-rotating terms (blue) with the RWA, 0-detuning analysis with Gaussian error propagation (red). 
simple model (see, for example, the points at $0.5,0.55,0.6,0.8$, and 0.9 ). This seems to suggest that some sources of error are correlated so as to reduce the $\left\langle\hat{X}_{1}^{2}\right\rangle$ error at higher ratios and to increase it at intermediate ratios. Both analyses find quantum squeezing at several ratios.

\subsection{Discussion}

From the Bayesian analysis, we infer a minimum $\left\langle\hat{X}_{1}^{2}\right\rangle$ value of $0.80 \pm 0.03\left\langle\hat{X}_{1}^{2}\right\rangle_{Z P}$, or $1.0 \pm 0.2 \mathrm{~dB}$ of sub-zero-point squeezing. At the same ratio, $\left\langle\hat{X}_{2}^{2}\right\rangle=10.95 \pm 0.72\left\langle\hat{X}_{1}^{2}\right\rangle_{Z P}$. The effective mechanical occupation, defined via $\left(n_{m}^{e f f}+1 / 2\right)^{2}=\left\langle\hat{X}_{1}^{2}\right\rangle\left\langle\hat{X}_{2}^{2}\right\rangle$ is thus 0.98 . Relative to this occupation, the $\hat{X}_{1}$ quadrature fluctuations are squeezed by $5.7 \mathrm{~dB}$, well over the $3 \mathrm{~dB}$ limit of parametric squeezing.

From Figs. 5.20 and 5.23, we see that both the cavity bath occupation and the mechanical loss rate increase with increasing ratio. The squeezing estimates derived from cooling measurements in Fig. 5.7 predict that optimum squeezing should occur at ratios above 0.6 at these pump powers. Instead, we obtain optimum squeezing at a ratio of $0.4-0.45$. Clearly, the amount of squeezing is limited by this ratio-dependent heating. This heating is further discussed in Appendix B.

The results presented in this chapter are the first evidence for quantum squeezing in a mechanical resonator. While our fits may not be a "direct" measurement of the squeezed state (i.e., we do not measure a signal directly proportional to $\left\langle\hat{X}_{1}^{2}\right\rangle$ as we do in Chapter 4$)$, if we assume that the linearized Hamiltonian described in Chapter 2 accurately describes our system, the measured output spectrum in the presence of the squeezing pumps tells us that the mechanics is in a squeezed state with $\hat{X}_{1}$ fluctuations below the zero-point level. The same linearized Hamiltonian has been shown to capture the behavior of many opto/electromechanical systems (it is assumed in most of the opto/electromechanics papers discussed in Chapter 1), including our own [51, 60]. The same approach of fitting the output spectrum and extracting the thermal bath occupations that we use here was also used to infer sideband cooling below an occupation of 1 in the widely-cited work by Teufel et. al. [56].

In the future, devices could be fabricated where the mechanics are coupled to two microwave cavities - one for squeezing and one for read-out. A double-cavity approach would avoid the problem of fitting the squeezing sideband and BAE probe sideband in the same cavity bandwidth. Coupling the mechanics to two microwave cavities while preserving high optomechanical coupling to both is likely to prove challenging, however.

In the KMC squeezing method, the cavity noise is also squeezed, and can be used to improve the imprecision of position detection [31]. Given the success of this method in squeezing the mechanical motion, measuring the squeezed cavity noise would be an obvious subject for future work. 


\section{Appendix A}

\section{Table of variable definitions}

\begin{tabular}{|c|c|}
\hline Symbol & Definition \\
\hline \multicolumn{2}{|r|}{ Frequencies } \\
\hline$\omega_{m}$ & mechanical frequency. For measurements, sometimes $f_{m}=\omega_{m} / 2 \pi$ is used \\
\hline$\gamma_{m}$ & mechanical loss/damping rate \\
\hline$\omega_{c}$ & cavity frequency. For measurements, sometimes $f_{c}=\omega_{c} / 2 \pi$ is used \\
\hline$\kappa_{L}, \kappa_{R}, \kappa_{\text {int }}$ & cavity loss rates for the left, right, and internal ports \\
\hline$\kappa$ & total cavity loss rate: $\kappa=\kappa_{L}+\kappa_{R}+\kappa_{\text {int }}$ \\
\hline$\omega_{-}, \omega_{+}$ & red- and blue-detuned pump frequencies \\
\hline$\Delta$ & $\begin{array}{l}\text { detuning between the mean pump frequency and the cavity frequency: } \\
\Delta=\left(\omega_{-}+\omega_{+}\right) / 2-\omega_{c}\end{array}$ \\
\hline$\delta$ & $\begin{array}{l}\text { detuning between half the pump frequency difference and the mechanical } \\
\text { frequency: } \delta=\left(\omega_{+}-\omega_{-}\right) / 2-\omega_{m}\end{array}$ \\
\hline$\Omega_{c}$ & mean pump frequency: $\Omega_{c}=\omega_{c}+\Delta=\left(\omega_{+}-\omega_{-}\right) / 2$ \\
\hline$g_{0}$ & single-photon optomechanical coupling rate \\
\hline$G_{-}, G_{+}$ & $\begin{array}{l}\text { red- and blue-detuned enhanced optomechanical coupling rates: } \\
G_{ \pm}=g_{0} \sqrt{n_{p}^{ \pm}}\end{array}$ \\
\hline $\mathcal{G}$ & effective enhanced optomechanical coupling rate: $\mathcal{G}^{2}=G_{-}^{2}-G_{+}^{2}$ \\
\hline $\mathcal{G}_{\text {tot }}$ & total enhanced optomechanical coupling rate: $\mathcal{G}^{2}=G_{-}^{2}+G_{+}^{2}$ \\
\hline$\gamma_{o p}^{-}, \gamma_{o p}^{+}$ & red- and blue-detuned optomechanical damping rates: $\gamma_{o p}^{ \pm}=4 g_{0}^{2} n_{p}^{ \pm} / \kappa$ \\
\hline$\gamma_{t o t}$ & total mechanical linewidth: $\gamma_{t o t}=\gamma_{m}+\gamma_{o p}^{-}-\gamma_{o p}^{+}$ \\
\hline \multicolumn{2}{|r|}{ Occupations } \\
\hline$n_{m}^{t h}$ & mechanical thermal bath occupation \\
\hline$\dot{n}_{m}^{t h}$ & mechanical thermal bath heating rate (in quanta/s): $\dot{n}_{m}^{t h}=\gamma_{m} n_{m}^{t h}$ \\
\hline
\end{tabular}


$n_{c, L}^{t h} \quad$ microwave thermal bath occupation at the left port of the cavity

$n_{c, R}^{t h} \quad$ microwave thermal bath occupation at the right port of the cavity

$n_{c, \text { int }}^{\text {th }} \quad$ microwave thermal bath occupation due to internal losses in the cavity

$n_{c}^{t h} \quad$ effective cavity thermal bath occupation: $n_{c}^{t h}=\Sigma_{i} \frac{\kappa_{i}}{\kappa} n_{c, i}^{t h}$

$\bar{n}_{m} \quad$ average mechanical occupation

$n_{p}^{-}, n_{p}^{+} \quad$ red- and blue-detuned pump occupations

\begin{tabular}{cl}
\hline & \multicolumn{1}{c}{ Measured quantities } \\
\hline$S_{21}$ & Complex transmission \\
& Noise spectral density measured at the output of the measurement chain. \\
& $(\mathrm{W} / \mathrm{Hz})$ \\
& Any noise spectral density that is independent of frequency over the mea- \\
& sured frequency span. $S_{0}$ can be referenced to the output of the device, or \\
& to the output of the measurement chain. \\
& Pump tone power measured at the output of the measurement chain. \pm \\
$P_{\text {thru }}$ & superscripts can be used to indicate measurements of the blue-detuned or \\
& red-detuned pump. (W) \\
& \\
\hline$\chi_{c}[\omega]$ & Cavity susceptibility: $\chi_{c}[\omega]=(\kappa / 2-i \omega)^{-1}$ \\
$\chi_{m}[\omega]$ & Mechanical susceptibility: $\chi_{m}[\omega]=\left(\gamma_{m} / 2-i \omega\right)^{-1}$ \\
$h[\omega]$ & See Eq. 2.33 (too long to reproduce) \\
$f[\omega]$ & See Eq. $2.44($ too long to reproduce $)$ \\
$g[\omega]$ & $g[\omega]=\mathcal{G}^{2}+\left(\gamma_{m} / 2-i \omega\right)(\kappa / 2-i \omega)($ Eq. 2.51$)$ \\
$\tilde{g}[\omega]$ & $g[\omega]=\mathcal{G}^{2}+\left(\gamma_{m} / 2-i \omega\right)(\kappa / 2-i \Delta)($ Eq. 2.63$)$ \\
$g_{-}[\omega]$ & $g[\omega]=\mathcal{G}^{2}+\left(\gamma_{m} / 2-i \omega\right)(\kappa / 2-i(\omega+\Delta))($ Eq. 2.68$)$ \\
\hline &
\end{tabular}




\section{Appendix B}

\section{Power-dependent heating}

For both D1 and D2, the amount of squeezing is limited by the presence of power-dependent heating of the cavity and mechanical baths. Similarly, heating of the cavity bath in the presence of a single red-detuned tone has prevented superconducting electromechanical devices from sideband cooling deep into the ground state $[45,56,36]$. This heating is typically assumed to be due to two-level systems (TLS) in the oxide at the surface of the superconductor. Signatures of TLS noise include improvement of the electrical quality factor at higher pump powers due to TLS saturation and a shifting cavity frequency with pump power due to the changing effective dielectric constant of the TLS [52]. We observe such effects for most of our devices (see, for example, $\kappa$ and $\omega_{c}$ vs. $n_{p}^{-}$in Fig. 5.4). The presence of TLS is known to effectively increase $n_{c}^{t h}$, as TLS noise changes the dielectric constant in the microwave cavity, and thus induces frequency noise in the cavity frequency. The presence of the pump converts this frequency noise to phase noise on the pump, which then looks like thermal cavity noise. The effective cavity noise, $n_{c}^{t h}$, is expected to be proportional to $\sqrt{n_{p}}$ [52]. Our cooling measurements for D2 roughly show this dependence (Fig. 5.6). Decreasing the contributions of TLS noise through fabrication techniques is a current goal of the Schwab group.

In the experiments discussed here, we not only observe heating that increases with the total pump power, but also heating that is dependent on the pump ratio (Figs. 5.20 and 5.23). Two-level systems in microwave resonators are not well-studied in the presence of two strong drive tones of similar power, so the physical mechanism behind this ratio-dependent noise is currently unknown. In this Appendix, I will try to characterize this noise with the hopes that we will be able to find an explanation in the future.

\section{B.1 $n_{c}^{\text {th }}$ heating in the absence of mechanics}

To explore and characterize the behavior of the ratio-dependent heating, we can first study the cavity heating alone by moving the mechanical sidebands out of the cavity and measuring only the cavity occupation vs. pump ratio. When the pump power ratio approaches and surpasses 1 at 


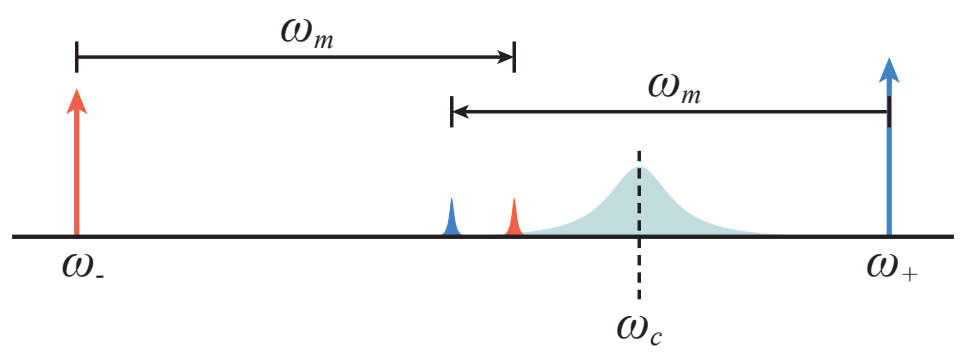

Figure B.1: Pump configuration for studying $n_{c}^{\text {th }}$ heating. This configuration ensures that the cavity enhancement of any sidebands of the blue-detuned pump is less than the cavity enhancement for the corresponding sideband of the red-detuned pump. For the measurements in Fig. B.2 and Fig. B.3, $\omega_{-}=\omega_{c}-2 \pi \times 4.5 \mathrm{MHz}$, and $\omega_{+}=\omega_{c}+2 \pi \times 2 \mathrm{MHz}$.

high pump powers, the mechanical linewidth narrows considerably and the mechanical motion is amplified, even when the pumps are equally detuned so that their sidebands are far from $\omega_{c}$ (i.e., $\delta>\kappa)$. The mechanical mode can then become unstable, as it is susceptible to frequency shifts on the scale of the mechanical linewidth. This instability will set in at lower pump powers if any of the blue-detuned pump's sidebands - from the fundamental or from any higher-order mode of the mechanics - are closer to the center of the cavity than the corresponding red-detuned pump's sideband, as the cavity enhances scattering between the blue-detuned pump and the mechanical mode. In order to explore the cavity heating as the pump ratio approaches or surpasses 1 , it is thus necessary to position the pumps so that all mechanical mode sidebands are outside the cavity bandwidth and, at the same time, the blue-detuned pump's sidebands are all further from the cavity than the red-detuned pump's. Fig. B.1 shows one such configuration.

Using this configuration, we can turn up the blue pump power to twice the red power without unstable mechanics dominating the spectrum. We change the total power of the applied pumps as well as the pump power ratio and measure the thermal cavity occupation at each pump configuration. Fig. B.2 shows the extracted $n_{c}^{t h}$ occupation as a function of both total pump power and power ratio. As the total pump power increases, $n_{c}^{t h}$ increases slightly. As the ratio changes, however, $n_{c}^{\text {th }}$ peaks strongly at a ratio of 1 before decreasing again. At the higher pump powers, $n_{c}^{\text {th }}$ at a ratio of 1 is about 4-5 times greater than it is at a ratio of 0. Fig. B.2 shows us that the excess heating is not simply a result of increasing the blue power. Instead, it seems that the heating is greatest when the pump powers are equal. When the pump powers are equal, the power in the cavity beating at $2 \omega_{m}$ is maximized, so the heating is likely related to this low-frequency beating.

As the thermal conductance between the cavity bath and the fridge may be smaller at low temperatures, we tried repeating this measurement at different fridge temperatures to see if we could reduce $n_{c}^{t h}$. Fig. B.3a shows $n_{c}^{t h}$ vs. pump ratio at a fixed total pump power of $n_{p}^{-}+n_{p}^{+}=1.3 \times 10^{7}$ at temperatures of $10 \mathrm{mK}, 100 \mathrm{mK}, 200 \mathrm{mK}$, and $400 \mathrm{mK}$. Raising the sample temperature only 


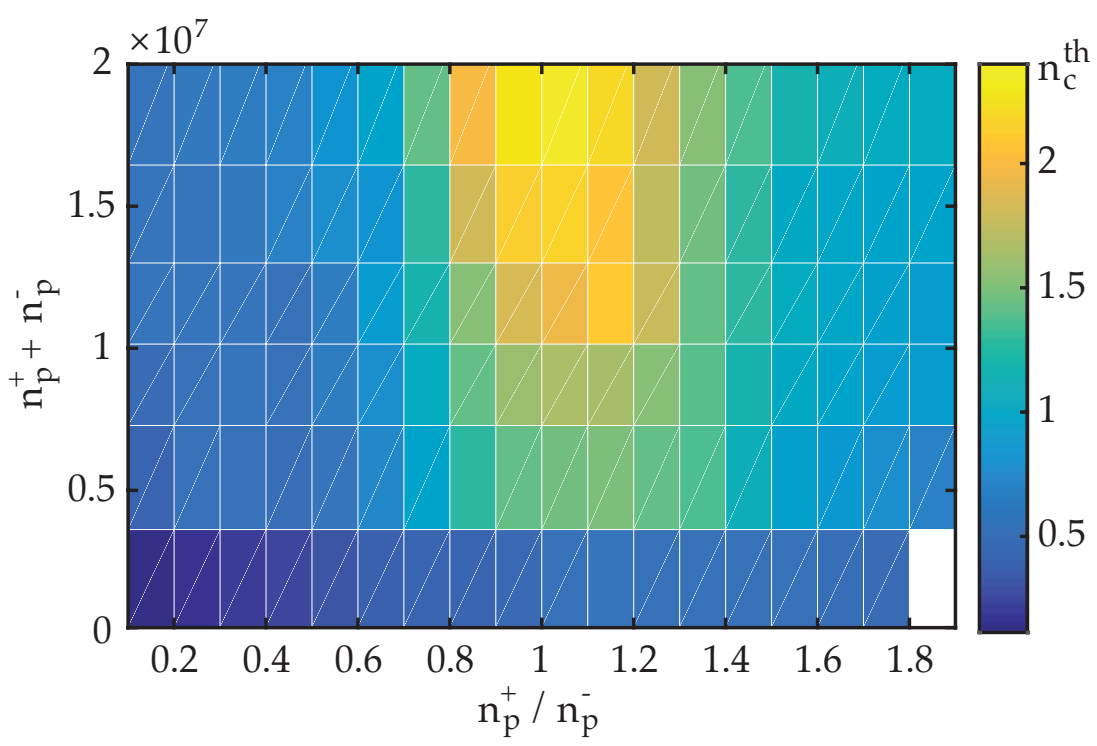

Figure B.2: $n_{c}^{\text {th }}$ vs. pump power ratio (x-axis) and total pump power (y-axis). $n_{c}^{\text {th }}$ ranges from 0.1 (dark blue) to 2.4 (yellow), with maximum heating at high pump powers with a ratio of 1.

seems to make the noise worse. As the thermal cavity occupation given by the Bose distribution is expected to increase from effectively 0 at $10 \mathrm{mK}$ to $\sim 0.9$ at $400 \mathrm{mK}$, some of this increase in noise may be due to the increase in sample temperature. In Fig. B.3b, the Bose occupation is subtracted off, but there is still some temperature dependence at all ratios, especially at temperatures over 100 $\mathrm{mK}$.

Cavity heating vs. pump ratio is not present when both pumps are red-detuned from the cavity, as seen in Fig. B.4. Here, one tone is located at $\omega_{c}-2 \pi \times 11 \mathrm{MHz}$, and one is located at $\omega_{c}-2 \pi \times 4.5$ $\mathrm{MHz}$. The pumps have the same spacing of $6.5 \mathrm{MHz}$ as in Fig. B.2, and thus produce beat power at the same frequency as before.

\section{B.2 3-omega}

By adding a third tone located at $\omega_{c}-3 \omega_{m}$, it is possible to adjust the amplitude and phase of the third tone to cancel out the power beating at $2 \omega_{m}$ (Fig. B.5) [50]. We can easily add a third tone and adjust its phase using the vector source, Agilent E8267C. We thus don't have to use the phase measurement set-up in Section 4.3.1.

We chose the total $n_{p}^{-}+n_{p}^{+}$to be $1.5 \mathrm{e} 7$, with a ratio of $n_{p}^{+} / n_{p}^{-}=0.68$. The 3 -omega tone has the same intracavity power as the blue-detuned tone. Fig. B.6 shows the spectra we obtain for different phases of the third tone. Even by eye, it is clear that the cavity and mechanical occupations are dependent on the third tone's phase. 

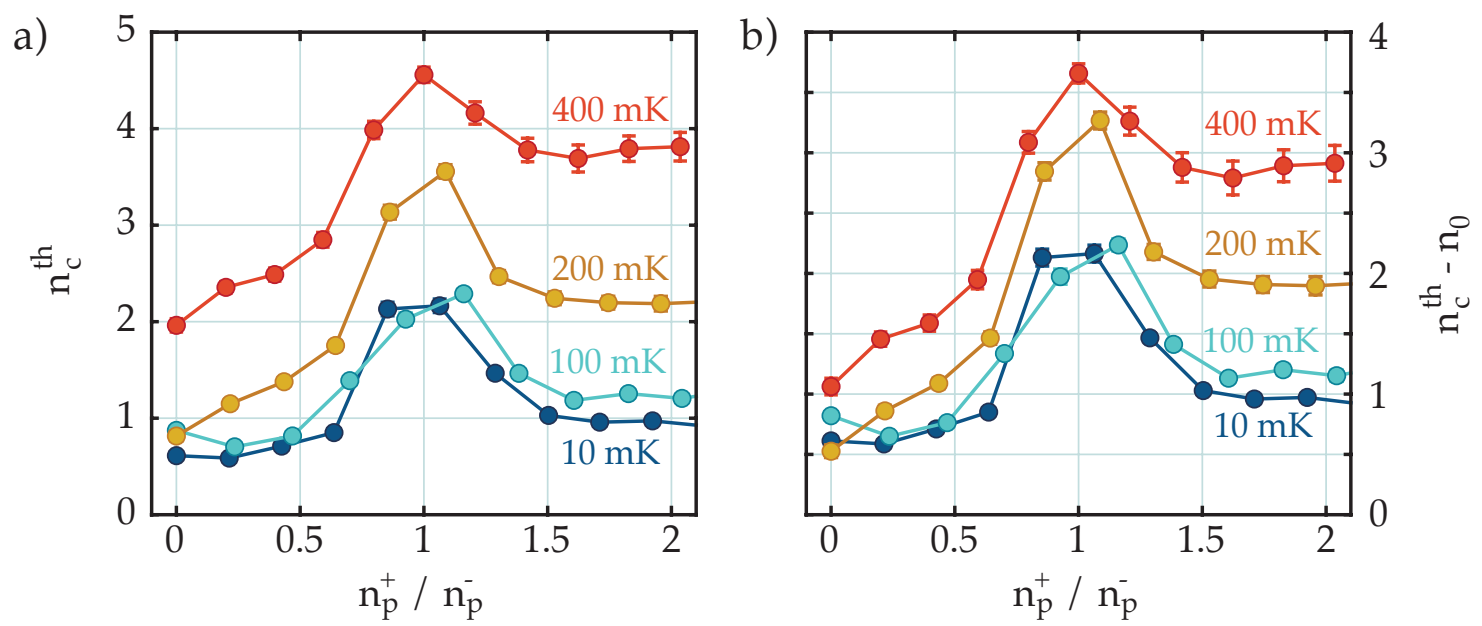

Figure B.3: a) $n_{c}^{\text {th }}$ vs. pump power ratio for different temperatures at a fixed total power of $n_{p}^{-}+n_{p}^{+}=1.3 \times 10^{7}$. b) Same as (a), but with $n_{0}=\left(e^{\hbar \omega_{c} / k_{B} T}-1\right)^{-1}$ subtracted from $n_{c}^{t h}$ at each temperature $T$.

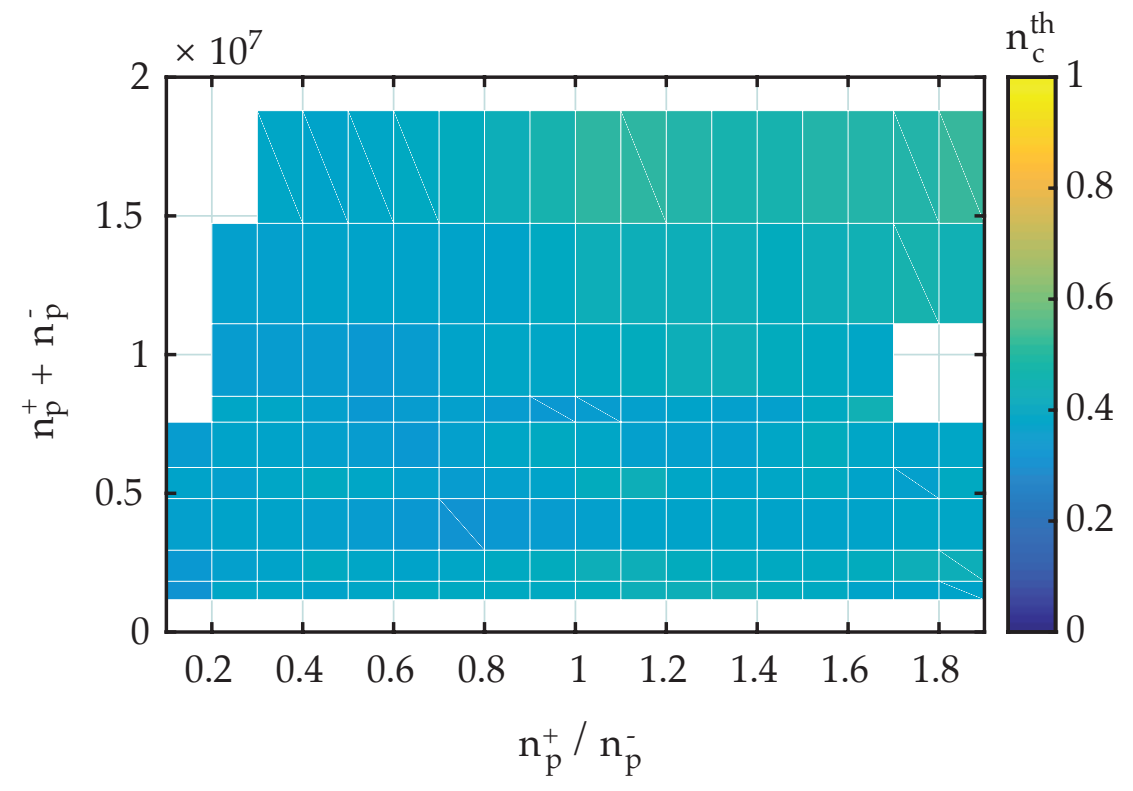

Figure B.4: $n_{c}^{\text {th }}$ heating map for two red-detuned tones. $n_{c}^{\text {th }}$ is shown vs. the pump power ratio (x-axis), and total pump power (y-axis). $\omega_{-}=\omega_{c}-2 \pi \times 11 \mathrm{MHz}$, and $\omega_{+}=\omega_{c}-2 \pi \times 4.5 \mathrm{MHz}$. No ratio-dependent heating is evident. 


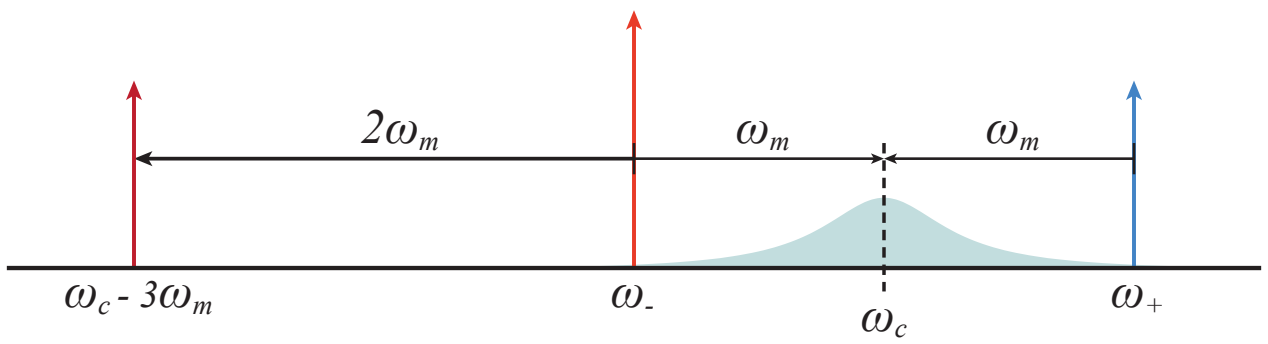

Figure B.5: Frequency configuration for 3 omega cancellation. A third tone is introduced at $\omega_{c}-3 \omega_{m}$ with the intracavity occupation as the blue-detuned tone.
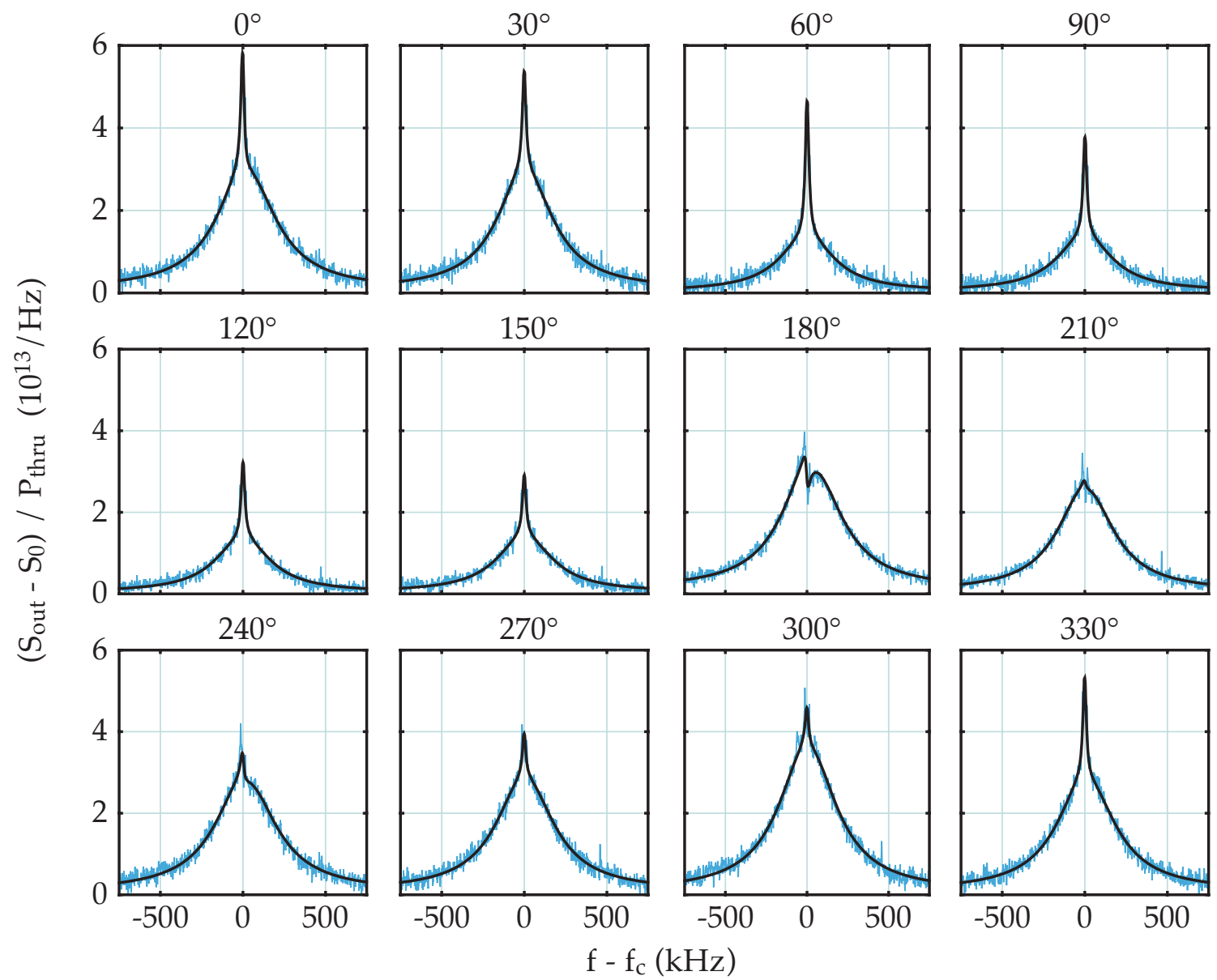

Figure B.6: Squeezing spectra at different 3-omega tone phases. The spectral response changes drastically vs. phase, despite the pump powers remaining constant throughout. Fits include a non-zero $\Delta$. 

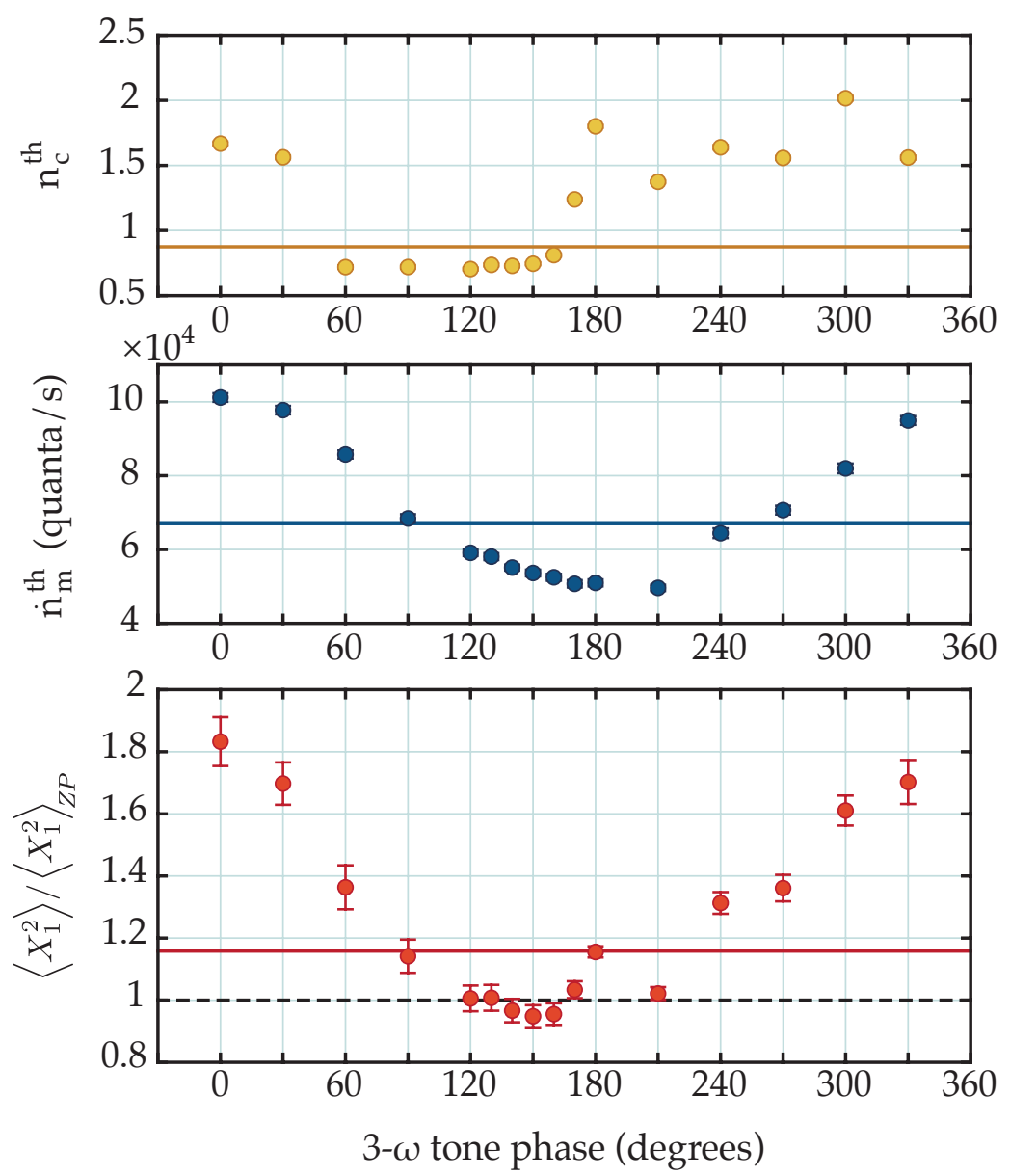

Figure B.7: Bath occupations and $\hat{X}_{1}$ variance vs. 3-omega phase. Solid lines indicate fit parameters in the presence of two tones, without the 3-omega tone added. Both bath occupations appear to decrease and increase relative to the two-tone values. The phase where the cavity occupation is minimized is not the same as the minimum phase for the mechanics. Optimum squeezing occurs at a phase in between the minimum phases for the cavity and the mechanics.

Fig. B.7 shows the fit results extracted from the spectra in Fig. B.6. Both $n_{c}^{\text {th }}$ and $\dot{n}_{m}^{\text {th }}$ show a dependence on phase. Relative to the bath occupations in the presence of two pumps, both also show a reduction at some phases and an increase for others. This adds additional support to the theory that the heating is due to the power in the cavity beating at $2 \omega_{m}$. Adding a third tone at $\omega_{c}-3 \omega_{m}$ with the correct phase relation may thus help offset the effects of ratio-dependent heating in future measurements. 


\section{Bibliography}

[1] J. Aasi and et. al. Enhanced sensitivity of the LIGO gravitational wave detector by using squeezed states of light. Nature Photonics, 7(8):613-619, 2013.

[2] B. P. et. al. Abbott. LIGO: the Laser Interferometer Gravitational-Wave Observatory. Reports on Progress in Physics, 72(7):076901, July 2009.

[3] T. Accadia, F. Acernese, F. Antonucci, P. Astone, G. Ballardin, F. Barone, M. Barsuglia, A. Basti, T. S. Bauer, and M. G. Beker. Calibration and sensitivity of the Virgo detector during its second science run. Classical and Quantum Gravity, 28(2):025005, 2011.

[4] G. Anetsberger, E. Gavartin, O. Arcizet, Q. P. Unterreithmeier, E. M. Weig, M. L. Gorodetsky, J. P. Kotthaus, and T. J. Kippenberg. Measuring nanomechanical motion with an imprecision below the standard quantum limit. Physical Review A, 82(6):61804, December 2010.

[5] M. Asjad, G. S. Agarwal, M. S. Kim, P. Tombesi, G. Di Giuseppe, and D. Vitali. Robust stationary mechanical squeezing in a kicked quadratic optomechanical system. Physical Review A, 89(2):023849, February 2014.

[6] P. Astone, M. Bassan, P. Bonifazi, F. Bronzini, M. G. Castellano, and others. First cooling below $0.1-\mathrm{K}$ of the new gravitational wave antenna 'Nautilus' of the Rome group. Europhysics Letters, 16:231-235, 1991.

[7] V. B. Braginski and A. B. Manukin. Ponderomotive effects of electromagnetic radiation. Soviet Physics JETP, 25:653, 1967.

[8] V. B. Braginsky, Y. I. Vorontsov, and K. S. Thorne. Quantum nondemolition measurements. Science, 209(4456):547-557, 1980.

[9] C. M. Caves. Quantum-mechanical radiation-pressure fluctuations in an interferometer. Physical Review Letters, 45(2):75-79, July 1980.

[10] J. Chan, T. P. M Alegre, A. H. Safavi-Naeini, J. T. Hill, A. Krause, S. Gröblacher, M. Aspelmeyer, and O. Painter. Laser cooling of a nanomechanical oscillator into its quantum ground state. Nature, 478(7):89-92, October 2011. 
[11] J. I. Cirac, A. S. Parkins, R. Blatt, and P. Zoller. "Dark" squeezed states of the motion of a trapped ion. Physical Review Letters, 70(5):556-559, February 1993.

[12] A. N. Cleland and M. L. Roukes. A nanometre-scale mechanical electrometer. Nature, 392(6672):160-162, 1998.

[13] A. A. Clerk, S. M. Girvin, F. Marquardt, and R. J. Schoelkopf. Introduction to quantum noise, measurement, and amplification. Reviews of Modern Physics, 82(2):1155-1208, April 2010.

[14] A. A. Clerk, F. Marquardt, and K. Jacobs. Back-action evasion and squeezing of a mechanical resonator using a cavity detector. New Journal of Physics, 10(9):5010, September 2008.

[15] J. M. Dobrindt, I. Wilson-Rae, and T. J. Kippenberg. Parametric normal-mode splitting in cavity optomechanics. Physical Review Letters, 101(2):263602, December 2008.

[16] T. J. Dunn, J. N. Sweetser, I. A. Walmsley, and C. Radzewicz. Experimental determination of the dynamics of a molecular nuclear wave packet via the spectra of spontaneous emission. Physical Review Letters, 70(2):3388-3391, May 1993.

[17] D. Foreman-Mackey, D. W. Hogg, D. Lang, and J. Goodman. emcee: The mcmc hammer. Publications of the Astronomical Society of the Pacific, 125(925):306-312, 2013.

[18] J. Gao, M. Daal, A. Vayonakis, S. Kumar, J. Zmuidzinas, B. Sadoulet, B. A. Mazin, P. K. Day, and H. G. Leduc. Experimental evidence for a surface distribution of two-level systems in superconducting lithographed microwave resonators. Applied Physics Letters, 92(1):152505, April 2008.

[19] C. W. Gardiner and M. J. Collett. Input and output in damped quantum systems - Quantum stochastic differential equations and the master equation. Physical Review A, 31:3761-3774, June 1985.

[20] C. W. Gardiner and P. Zoller. Quantum noise: A handbook of Markovian and non-Markovian quantum stochastic methods with applications to quantum optics. Springer, 3 edition, 2004.

[21] G. A. Garrett, A. G. Rojo, A. K. Sood, J. F. Whitaker, and R. Merlin. Vacuum squeezing of solids: Macroscopic quantum states driven by light pulses. Science, 275(5306):1638-1640, 1997.

[22] C. Gerry and P. Knight. Introductory quantum optics. Cambridge University Press, November 2004

[23] W.-J. Gu, G. Li, and Y.-P. Yang. Generation of squeezed states in a movable mirror via dissipative optomechanical coupling. Physical Review A, 88(1):013835, July 2013. 
[24] J. Hald, J. L. Sørensen, C. Schori, and E. S. Polzik. Spin squeezed atoms: A macroscopic entangled ensemble created by light. Physical Review Letters, 83(7):1319-1322, August 1999.

[25] J. B. Hertzberg. Back-action evading measurements of nanomechanical motion approaching quantum limits. PhD thesis, University of Maryland, 2009.

[26] U. B. Hoff, G. I. Harris, L. S. Madsen, H. Kerdoncuff, M. Lassen, G. M. Nielsen, W. P. Bowen, and U. L. Andersen. Quantum-enhanced micromechanical displacement sensitivity. Optics Letters, 38:1413, May 2013.

[27] K. Jähne, C. Genes, K. Hammerer, M. Wallquist, E. Polzik, and P. Zoller. Cavity-assisted squeezing of a mechanical oscillator. Physical Review A, 79(6):063819, June 2009.

[28] D. Kienzler, H. Y. Lo, B. Keitch, L. de Clercq, F. Leupold, F. Lindenfelser, M. Marinelli, V. Negnevitsky, and J. P. Home. Quantum harmonic oscillator state synthesis by reservoir engineering. Science, 347(6):53-56, January 2015.

[29] M. s. Kim, F. A. M. de Oliveira, and P. L. Knight. Properties of squeezed number states and squeezed thermal states. Physical Review A, 40:2494-2503, 1989.

[30] A. Kronwald, F. Marquardt, and A. A. Clerk. Arbitrarily large steady-state bosonic squeezing via dissipation. Physical Review A, 88(6):63833, December 2013.

[31] A. Kronwald, F. Marquardt, and A. A. Clerk. Dissipative optomechanical squeezing of light. New Journal of Physics, 16(6):063058, 2014.

[32] F. Lecocq, J. D. Teufel, J. Aumentado, and R. W. Simmonds. Resolving the vacuum fluctuations of an optomechanical system using an artificial atom. arXiv.org, page 872, September 2014.

[33] X.-Y. Lü, J.-Q. Liao, L. Tian, and F. Nori. Steady-state mechanical squeezing in an optomechanical system via Duffing nonlinearity. Physical Review A, 91(1):013834, January 2015.

[34] A. Mari and J. Eisert. Gently modulating optomechanical systems. Physical Review Letters, 103(2):213603, November 2009.

[35] F. Marquardt, J. P. Chen, A. A. Clerk, and S. M. Girvin. Quantum theory of cavity-assisted sideband cooling of mechanical motion. Physical Review Letters, 99(9):93902, August 2007.

[36] F. Massel, S. U. Cho, J.-M. Pirkkalainen, P. J. Hakonen, T. T. Heikkilä, and M. A. Sillanpää. Multimode circuit optomechanics near the quantum limit. Nature Communications, 3:987, August 2012 .

[37] D. M. Meekhof, C. Monroe, B. E. King, W. M. Itano, and D. J. Wineland. Generation of nonclassical motional states of a trapped atom. Physical Review Letters, 76:1796-1799, 1996. 
[38] S. M. Meenehan, J. D. Cohen, G. S. MacCabe, F. Marsili, M. D. Shaw, and O. Painter. Pulsed excitation dynamics of an optomechanical crystal resonator near its quantum ground-state of motion. arXiv.org, page 5135, March 2015.

[39] A. D. O'Connell, M. Hofheinz, M. Ansmann, R. C. Bialczak, M. Lenander, E. Lucero, M. Neeley, D. Sank, H. Wang, M. Weides, J. Wenner, J. M. Martinis, and A. N. Cleland. Quantum ground state and single-phonon control of a mechanical resonator. Nature, 464(7289):697-703, March 2010.

[40] T. A. Palomaki, J. D. Teufel, R. W. Simmonds, and K. W. Lehnert. Entangling mechanical motion with microwave fields. Science, 342(6):710-713, November 2013.

[41] M. Poggio, C. L. Degen, H. J. Mamin, and D. Rugar. Feedback cooling of a cantilever's fundamental mode below 5 mK. Physical Review Letters, 99(1):017201-017201, July 2007.

[42] A. Pontin, M. Bonaldi, A. Borrielli, F. S. Cataliotti, F. Marino, G. A. Prodi, E. Serra, and F. Marin. Squeezing a thermal mechanical oscillator by stabilized parametric effect on the optical spring. Physical Review Letters, 112(2):23601, January 2014.

[43] T. P. Purdy, R. W. Peterson, and C. A. Regal. Observation of radiation pressure shot noise on a macroscopic object. Science, 339(6121):801-804, February 2013.

[44] P. Rabl, A. Shnirman, and P. Zoller. Generation of squeezed states of nanomechanical resonators by reservoir engineering. Physical Review B, 70(2):205304, November 2004.

[45] T. Rocheleau, T. Ndukum, C. Macklin, J. B. Hertzberg, A. A. Clerk, and K. C. Schwab. Preparation and detection of a mechanical resonator near the ground state of motion. Nature, 463(7):72-75, January 2010.

[46] D. Rugar, R. Budakian, H. J. Mamin, and B. W. Chui. Single spin detection by magnetic resonance force microscopy. Nature, 430(6997):329-332, 2004.

[47] D. Rugar and P. Gruetter. Mechanical parametric amplification and thermomechanical noise squeezing. Physical Review Letters, 67:699-702, August 1991.

[48] R. Ruskov, K. C. Schwab, and A. N. Korotkov. Squeezing of a nanomechanical resonator by quantum nondemolition measurement and feedback. Physical Review B, 71(2):235407, June 2005.

[49] R. E. Slusher, L. W. Hollberg, B. Yurke, J. C. Mertz, and J. F. Valley. Observation of squeezed states generated by four-wave mixing in an optical cavity. Physical Review Letters, 55:2409$2412,1985$. 
[50] S. K. Steinke, K. C. Schwab, and P. Meystre. Optomechanical backaction-evading measurement without parametric instability. Physical Review A, 88(2):023838, August 2013.

[51] J. Suh, A. J. Weinstein, C. U. Lei, E. E. Wollman, S. K. Steinke, P. Meystre, A. A. Clerk, and K. C. Schwab. Mechanically detecting and avoiding the quantum fluctuations of a microwave field. Science, 344(6):1262-1265, June 2014.

[52] J. Suh, A. J. Weinstein, and K. C. Schwab. Optomechanical effects of two-level systems in a back-action evading measurement of micro-mechanical motion. Applied Physics Letters, 103(5):2604, July 2013.

[53] A. Szorkovszky, G. A. Brawley, A. C. Doherty, and W. P. Bowen. Strong thermomechanical squeezing via weak measurement. Physical Review Letters, 110(1):184301, May 2013.

[54] A. Szorkovszky, A. C. Doherty, G. I. Harris, and W. P. Bowen. Mechanical squeezing via parametric amplification and weak measurement. Physical Review Letters, 107(2):213603, November 2011.

[55] J. D. Teufel, T. Donner, M. A. Castellanos-Beltran, J. W. Harlow, and K. W. Lehnert. Nanomechanical motion measured with an imprecision below that at the standard quantum limit. Nature Nanotechnology, 4(12):820-823, 2009.

[56] J. D. Teufel, T. Donner, Dale Li, J. W. Harlow, M. S. Allman, K. Cicak, A. J. Sirois, J. D. Whittaker, K. W. Lehnert, and R. W. Simmonds. Sideband cooling of micromechanical motion to the quantum ground state. Nature, 475(7356):359-363, 2011.

[57] M. R. Vanner, I. Pikovski, G. D. Cole, M. S. Kim, C. Brukner, K. Hammerer, G. J. Milburn, and M. Aspelmeyer. Pulsed quantum optomechanics. In Proceedings of the National Academy of Sciences, pages 16182-16187, September 2011.

[58] A. Vinante and P. Falferi. Feedback-enhanced parametric squeezing of mechanical motion. Physical Review Letters, 111(2):207203, November 2013.

[59] J. Weber. Evidence for discovery of gravitational radiation. Physical Review Letters, 22(24):1320, 1969.

[60] A. J. Weinstein, C. U. Lei, E. E. Wollman, J. Suh, A. Metelmann, A. A. Clerk, and K. C. Schwab. Observation and interpretation of motional sideband asymmetry in a quantum electromechanical device. Physical Review X, 4:041003, Oct 2014.

[61] T. Westphal, D. Friedrich, H. Kaufer, K. Yamamoto, S. Goßler, H. Müller-Ebhardt, S. L. Danilishin, F. Y. Khalili, K. Danzmann, and R. Schnabel. Interferometer readout noise below the standard quantum limit of a membrane. Physical Review A, 85(6):063806, June 2012. 
[62] T. White. Martinis Group Electronics Documentation: Cold SMA Switch, 2012 (accessed April 18, 2015). http://web.physics.ucsb.edu/ martinisgroup/electronics.shtml.

[63] B. Willke and et. al. The GEO 600 gravitational wave detector. Classical and Quantum Gravity, 19(7):1377-1387, April 2002.

[64] E. E. Wollman, C. U. Lei, A. Weinstein, J. Suh, A. Kronwald, F. Marquardt, A. A. Clerk, and K. C. Schwab. Evidence for quantum squeezing of motion in a mechanical resonator. In prep.

[65] N. Yazdi, F. Ayazi, and K. Najafi. Micromachined inertial sensors. Proceedings of the IEEE, 86(8):1640-1659, 1998.

[66] B. Yurke, P. G. Kaminsky, R. E. Miller, E. A. Whittaker, and A. D. Smith. Observation of 4.2K equilibrium-noise squeezing via a Josephson-parametric amplifier. Physical Review Letters, 60:764-767, February 1988.

[67] J. P. Zendri, L. Baggio, M. Bignotto, M. Bonaldi, M. Cerdonio, L. Conti, M. De Rosa, P. Falferi, P. L. Fortini, and M. Inguscio. Status report and near future prospects for the gravitational wave detector AURIGA. Classical and Quantum Gravity, 19(7):1925, 2002.

[68] J. Zhang, Y.-X. Liu, and F. Nori. Cooling and squeezing the fluctuations of a nanomechanical beam by indirect quantum feedback control. Physical Review A, 79(5):052102, 2009. 